\title{
CATÁLOGO TAXONÓMICO-GEOGRÁFICO DE LOS COLEÓPTEROS DE LA FAMILIA MELOIDAE DE MÉXICO
}

\author{
M. García-París ${ }^{1}$, D. Buckley ${ }^{1,3}$ y G. Parra-Olea ${ }^{2}$
}

\begin{abstract}
RESUMEN
Se presenta un catálogo taxonómico de los representantes mexicanos de la familia Meloidae que incluye un listado revisado de sinonimias, localidades típicas y registros geográficos publicados de cada una de las especies. La fauna mexicana incluye en la actualidad 255 especies vivientes y una fósil, distribuidas en 21 géneros de las subfamilias Meloinae, Nemognathinae y Tetraonycinae. En el último catálogo general, Blackwelder (1945) recogió la presencia en México de 160 especies de esta familia, casi 100 especies menos de las que se conocen en la actualidad y sin embargo, la cifra actual parece encontrarse aún lejos de la real, ya que existen muchas especies ampliamente distribuidas al norte de la frontera con los Estados Unidos cuya presencia es muy probable en territorio mexicano.

En cuanto a la taxonomía y nomenclatura de las especies, en este catálogo se propone el uso de los nombres Epicauta dugesi Werner, 1957 y Tegrodera erosa extincta Beauregard, 1890; se incluyen tres sinonimias nuevas de Nemognatha chrysomeloides (Linnaeus, 1763) (N. atra Beauregard, 1890; N. pallidicollis Beauregard, 1890 y N. violacea Beauregard, 1890) y otra de E. dugesi (Epicauta tamara Adams \& Selander, 1979); se designa lectotipo para Lytta koltzei var. minor Haag-Rutenberg, 1880 con el propósito de solucionar el problema taxonómico generado tras la designación inválida de lectotipo de $L$. $k$. var. cyanescens; y finalmente se consideran como especies posiblemente a excluir del listado de Meloidae de México a Cissites maculata (Swederus, 1787) y Tetraonyx (Tetraonyx) bimaculatus (Klug, 1825).
\end{abstract}

Palabras clave: Coleoptera, Meloidae, México, Taxonomía, Nomenclatura, Sinonimias, Faunística, América del Norte.

\section{ABSTRACT \\ Taxonomic and geographic catalogue of the Coleoptera belonging to the family Meloidae of Mexico}

A comprehensive taxonomic catalogue of the Mexican representatives of the family Meloidae is presented. The catalogue includes a revised synonymical list including type localities and published geographical records for all known species. The Mexican Fauna of Meloidae currently includes 255 extant species, plus one only known from the fossil

Museo Nacional de Ciencias Naturales. Consejo Superior de Investigaciones Científicas (CSIC). c/ José Gutiérrez Abascal, 2. 28006 Madrid. España.

2 Instituto de Biología. Universidad Autónoma de México (UNAM). AP 70-153, CP 04510. Ciudad Universitaria. México D.F. México.

3 Dpt. Integrative Biology \& Museum of Vertebrate Zoology, 3060 VLSB, University of California, Berkeley, CA 94720-3140. 
record, arranged in 21 genera within the subfamilies Meloinae, Nemognathinae and Tetraonycinae. The last comprehensive catalogue published (Blackwelder, 1945) recorded the presence of 160 species of Meloidae in México, almost 100 species less than the current known number. However the current number of species seems to be far below the actual number, since many species widely distributed along the northern border, within the United States, are likely to be found also in Mexico.

Regarding taxonomic and nomenclatural changes, in this catalogue we propose the use of the names Epicauta dugesi Werner, 1957 and Tegrodera erosa extincta Beauregard, 1890; we propose three new synonymies for Nemognatha chrysomeloides (Linnaeus, 1763) (N. atra Beauregard, 1890; N. pallidicollis Beauregard, 1890 and N. violacea Beauregard, 1890) and one for E. dugesi (Epicauta tamara Adams \& Selander, 1979); we designate lectotype for Lytta koltzei var. minor Haag-Rutenberg, 1880 with the goal of resolving the taxonomic problem generated by the previous invalid designation of lectotype for $L . k$. var. cyanescens; and finally we consider Cissites maculata (Swederus, 1787) and Tetraonyx (Tetraonyx) bimaculatus (Klug, 1825) as species to be possibly excluded from the Mexican checklist.

Key words: Coleoptera, Meloidae, Mexico, Taxonomy, Nomenclature, Sinonymy, Faunistic, North America.

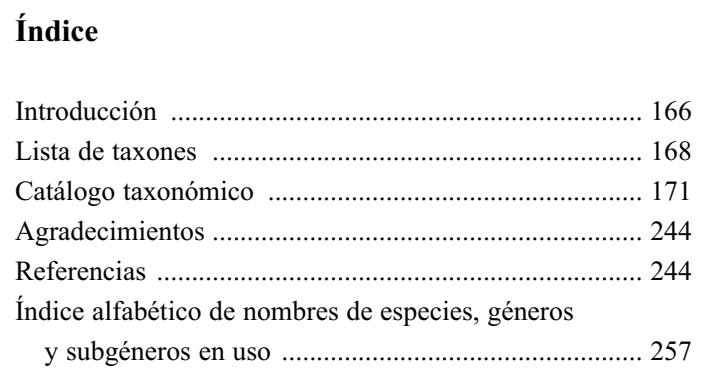

\section{Introducción}

La familia Meloidae cuenta con unos 120 géneros y 2500 especies distribuidas por la mayor parte del Planeta, excepto en Nueva Zelanda y en algunas islas oceánicas aisladas (Pinto \& Bologna, 1999). La presencia de altas concentraciones de cantaridina en los tejidos de los coleópteros de esta familia ha llamado la atención desde la antigüedad y desde entonces han sido utilizados por su interés farmacológico. De hecho, se trata de una de las pocas familias de insectos que poseen nombres vernáculos en la mayoría de las lenguas y que, a nivel de familia, tienen una denominación general como "escarabajos vesicantes" o "cantáridas". La ingestión accidental de estos coleópteros, que pueden ir incluidos en las balas y pacas de alfalfa (Blodgett et al., 1995), ocasiona trastornos graves o incluso la muerte al ganado, por lo que son objeto de atención en veterinaria (Schoeb \& Panciera, 1978; Capinera et al., 1985). También son conocidos en agricultura ya que algunas especies consituyen plagas ocasionales en su estado adulto, mientras que en estado larvario pueden tener utilidad agrícola como con- troladores de otras plagas (Dampf, 1925; Horsfall, 1943; Parker \& Wakeland, 1957). Otros aspectos de su biología, como sus ciclos biológicos complejos que incluyen hipermetamorfosis y hábitos larvarios parasitoides (Beauregard, 1890; Paoli, 1937; Selander, 1981), sus diversas pautas de cortejo y comportamiento gregario (Horsfall, 1943; Selander, 1960a, 1964a) y sus defensas químicas (Carrel \& Eisner, 1974; Carrel et al., 1993; Dettner, 1997) son excepcionales entre los coleópteros. En áreas donde la alteración ambiental es alta, o donde los usos agrícolas han cambiado, se ha detectado su progresiva desaparición (Nash, 1976; Havelka, 1980) y en los Estados Unidos se han propuesto 5 especies como candidatas para su inclusión en las listas de especies amenazadas a nivel federal (Halstead \& Haines, 1992).

La composición de la familia ha variado relativamente poco a través del tiempo. Pinto \& Bologna (1999) siguen a Aksentjev $(1988 ; 1989)$ y excluyen de la familia al género Thambospasta Werner, 1974, que había sido previamente incluido en la subfamilia Eleticinae (Werner, 1974). Las aportaciones más recientes al conocimiento de la sistemática del con- 
junto de la familia son las clasificaciones presentadas por Kaszab (1959, 1969), Bologna (1991), Selander (1991) y Bologna \& Pinto (2001). La familia se subdivide en 4 subfamilias: Eleticinae, Meloinae, Nemognathinae y Tetraonycinae. Los representantes de la subfamilia Eleticinae se distribuyen por América del Sur, África, La India y el sureste de Asia. La subfamilia Tetraonycinae es exclusiva de América, mientras que Meloinae y Nemognathinae están ampliamente distribuidos por casi todo el Planeta (Pinto \& Bologna, 1999).

Exceptuando la monumental obra de Beauregard (1890) y los catálogos y listados publicados en la primera mitad del siglo XX (Borchmann, 1917), no existen trabajos monográficos de conjunto para la familia. La fauna americana de Meloidae fue catalogada por Denier (1935a, 1940) y Blackwelder (1945) y recientemente, Pinto \& Bologna (1999) han publicado una clave de identificación y una revisión de todos los géneros presentes en América. En este continente se reconocen 49 géneros que incluyen unas 790 especies (Pinto \& Bologna, 1999). De estos géneros, únicamente 5 se encuentran también en el Viejo Mundo (Bologna \& Pinto, 2002).

Varias especies de Meloidae ya eran conocidas en México antes del inicio de los estudios taxonómicos, como lo atestigua la existencia de nombres vernáculos tanto en lenguas prehispánicas como en español ("panchitos meones"). Algunos de estos nombres han sido recogidos en la literatura científica como los que presentan Herrera \& Mendoza (1866) para Lytta eucera (Chevrolat, 1834): "tlayacuayatl" (nahuatl), "tungaxeni" (otomí) y "pipila ciega", o el que comenta Peñafiel Barranco (1866) para Meloe, posiblemente en Hidalgo: "tecushene". En cuanto al estudio taxonómico de los Meloidae de México, los primeros registros de ejemplares mexicanos se encuentran en la obra de Chevrolat $(1833,1834 \mathrm{a}, 1834 \mathrm{~b})$ y en los trabajos de Herrera \& Mendoza (1866; 1867), Jiménez (1866a, b) y Peñafiel Barranco (1866). Los primeros trabajos de conjunto son los de Dugès (1869a, 1869b, 1869c, 1870) y Champion $(1891,1892,1893)$, autores que ya incluyeron claves de identificación. A estos trabajos hay que añadir las descripciones de especies nuevas realizadas sobre ejemplares estadounidenses de especies que se encuentran también en México por LeConte $(1851,1853,1854 \mathrm{a}, 1854 \mathrm{~b}$, $1854 \mathrm{c}, 1854 \mathrm{~d}, 1857,1858 \mathrm{a}, 1858 \mathrm{~b}, 1866)$, Horn $(1870,1874,1883,1885,1891,1894,1895) \mathrm{y}$ Casey (1891). Ya bien entrado el siglo XX, Vaurie (1950), aunque con diversos problemas de asignación e identificación de especies, presenta y recopi- la datos sobre especies del norte de México. Además, mucha de la informacion publicada por Enns (1956) sobre Nemognathini de los EEUU y por Werner et al. (1966) sobre Arizona es útil para la identificación de las especies del norte de México. Varios de los géneros presentes en México, o parte de ellos, han sido objeto de estudios monográficos, que en muchos casos incluyen a toda la fauna de América del Norte. Tal es el caso de los géneros Lytta (Selander, 1960a) y Meloe (Pinto \& Selander, 1970), del subgénero nominal de Epicauta (Adams \& Selander, 1979; Pinto, 1991) y de los géneros de la tribu Eupomphini (Pinto, 1975a, 1977, 1979, 1984a,c). Finalmente, Pinto \& Bologna (1999) presentan claves de identificación de todos los géneros americanos e indican la distribución general de cada uno de ellos. A pesar de estas contribuciones, el grado de conocimiento de la fauna de México de Meloidae es limitado y prueba de ello es que, por ejemplo, en el listado "Nomina Insecta Nearctica" (Poole \& Gentili, 1996) se han omitido la mayor parte de las especies mexicanas de Meloidae, a pesar de pertenecer en su mayor parte a la fauna neártica.

Eugenio Dugès fue sin duda el precursor del estudio moderno de los meloidos de México y sus publicaciones dedicadas a este grupo (1869a, $1869 \mathrm{~b}, 1869 \mathrm{c}, 1870,1874 \mathrm{a}, 1874 \mathrm{~b}, 1877,1881$, 1886a, 1886b, 1889a, 1889b, 1889c, 1889d, 1889e, 1889f), junto con la obra coetánea de Champion (1891, 1892, 1893), proporcionaron la base sobre la que se asentarían los estudios posteriores. Desafortunadamente, la colección de ejemplares de Dugès, incluidos muchos de sus tipos, ha sufrido numerosos traslados y pérdidas significativas, hasta llegar a su localización actual en la Colección Nacional de Insectos del Instituto de Biología de la Universidad Nacional Autónoma de México (Zaragoza Caballero, 1999). Como consecuencia, se ha requerido la designación de neotipos para muchas de las especies de Dugès, que en la mayoría de los casos se localizan en instituciones fuera de México. Horn (1885) comenta que recibió para estudio los tipos de E. ocellata (Dugès, 1869) y de otras especies de Meloidae descritas por Dugès, por lo que habría que revisar si éstos aún se encuentran en la colección Horn del Museum of Comparative Zoology (Harvard University, Cambridge, EEUU). Zaragoza Caballero (1999) delinea la complicada historia de la colección Dugès y además comenta la obra, en gran parte aún inédita, de este investigador.

En el último catálogo general, Blackwelder (1945) recoge la presencia en México de 160 especies de la familia Meloidae, repartidas en 18 géne- 
ros. En la actualidad, en México se conocen un total de 255 especies que se distribuyen en tres subfamilias: Meloinae, Tetraonycinae y Nemognathinae. Además se ha registrado una especie fósil del género Epicauta (Zaragoza-Caballero \& Velasco-de León, 2001, 2003).

La nomenclatura supragenérica utilizada en este catálogo sigue la propuesta de Bologna (1991) y Pinto \& Bologna (1999), por lo que en el listado sinonímico no se han incluido las sinonimias de los taxones supragenéricos, aunque como se señala en otros trabajos, éstas todavía presentan problemas sin resolver (ver Mulsant, 1857; Wellman, 1910a; Cros, 1940; Aksentjev, 1988, 1989; Selander, 1955b, 1991; Bologna \& Pinto, 2001, 2002). En cuanto a la nomenclatura de las especies, ha sido necesario proponer algunos cambios de nombres, bien debido a la existencia de problemas en los listados sinonímicos anteriores que no se ajustaban al Código Internacional de Nomenclatura Zoológica (CINZ, 2000), como el caso de Epicauta dugesi Werner, 1957, o bien debidos a la revisión comparada de las descripciones originales, como en el caso de Tegrodera erosa extincta Beauregard, 1890. Además, incluimos tres sinonimias nuevas para Nemognatha chrysomeloides (Linnaeus, 1763), una especie muy variable, que en realidad podría estar constituida por un complejo de taxones bien diferenciados a lo largo de su enorme distribución geográfica y otra para $E$. dugesi. Es de reseñar que muchas de las combinaciones nomenclaturales atribuidas generalmente a Champion $(1891,1892,1893)$ o a autores posteriores, corresponden en realidad a Beauregard (1890). En las sinonimias se señalan con un asterisco (*) los nombres revisados en su publicación original. Los errores tipográficos en la nomenclatura se incluyen en el epígrafe de comentarios taxonómicos y no en el listado sinonímico.

Para la confección de los epígrafes de distribución geográfica se han utilizado únicamente registros publicados, por lo tanto, a no ser que los datos se incluyeran en otros trabajos, no se han considerado las tesis inéditas de Hernández (1960) y Radford (1960), obras que, precisamente por tratar faunas locales aún poco estudiadas (Baja California y Nuevo León), merece la pena revisar con detalle. En los listados, únicamente se han incluido los registros estatales sin localidad precisa cuando no existen otros datos disponibles. El listado de citas pretende reflejar el estado de conocimiento de la distribución geográfica de cada especie y por lo tanto, los errores tipográficos obvios y algunos de los errores ortográficos, tanto de las publicaciones revisadas, como de las etiquetas de los ejemplares citados en las diferentes obras, se han tratado de corregir de acuerdo con la toponimia actual. Muchos de estos errores se nos habrán pasado por alto y en algunos casos dudosos se indica la grafía original y entre corchetes [], nuestra interpretación. Los corchetes en las localidades típicas figuran tal como se presentan en la obra original. El trabajo de Selander \& Vaurie (1962) ha sido especialmente útil para la localización de localidades clásicas. Las millas no se han transformado en kilómetros y en general, no se indican los datos de altitud.

\section{Lista de taxones}

En la lista que se presenta se han incluido 255 especies, pero la lista de especies presentes en México habrá de ampliarse considerablemente con especies citadas muy cerca de la frontera en California, Arizona, Nuevo México y Texas, pero que hasta el momento sólo han sido registradas en territorio de los Estados Unidos. La presencia en México de los géneros Spastonyx Selander, 1954 (Meloini) y Denierota Kaszab, 1959 (Pyrotini) se recoge en la revisión de Pinto \& Bologna (1999), pero en esa obra no se menciona ni especie ni localidad concreta alguna, por lo que en el catálogo no se ha incluido ninguna especie de estos géneros.

Familia Meloidae Gyllenhal, 1810

Subfamilia Meloinae Ganglbauer, 1907

Tribu Epicautini Denier, 1935

Género Epicauta Dejean, 1834

$\dagger$ Epicauta sanctoruensis Zaragoza-Caballero \& Velasco-de León, 2003

Subgénero Epicauta Dejean, 1834

Epicauta (Epicauta) abadona Skinner, 1904

Epicauta (Epicauta) abeona Pinto, 1980

Epicauta (Epicauta) afoveata Werner, 1949

Epicauta (Epicauta) albolineata (Dugès, 1877)

Epicauta (Epicauta) andersoni Werner, 1944

Epicauta (Epicauta) apache Pinto, 1980

Epicauta (Epicauta) atrata (Fabricius, 1775)

Epicauta (Epicauta) basimacula (Haag-Rutenberg, 1880)

Epicauta (Epicauta) bispinosa Werner, 1949

Epicauta (Epicauta) brunnea Werner, 1944

Epicauta (Epicauta) callosa LeConte, 1866

Epicauta (Epicauta) candidata Champion, 1892

Epicauta (Epicauta) cardui (Dugès, 1889)

Epicauta (Epicauta) carmelita (Haag-Rutenberg, 1880)

Epicauta (Epicauta) castadiva Pinto, 1991

Epicauta (Epicauta) cazieri Dillon, 1952 
Epicauta (Epicauta) cinctipennis (Chevrolat, 1834) Epicauta (Epicauta) conferta (Say, 1824) Epicauta (Epicauta) corvina (LeConte, 1858) Epicauta (Epicauta) costata (LeConte, 1854) Epicauta (Epicauta) crassitarsis Maydell, 1935 Epicauta (Epicauta) cupraeola (Dugès, 1869) Epicauta (Epicauta) curvicornis (Haag-Rutenberg, 1880) Epicauta (Epicauta) delicata Mathieu, 1983 Epicauta (Epicauta) diana Pinto, 1991 Epicauta (Epicauta) dugesi Werner, 1957 Epicauta (Epicauta) emarginata Champion, 1892 Epicauta (Epicauta) ferruginea (Say, 1824) Epicauta (Epicauta) fortis Werner, 1944 Epicauta (Epicauta) horni Champion, 1892 Epicauta (Epicauta) hubbelli Werner, 1973 Epicauta (Epicauta) insueta Werner, 1955 Epicauta (Epicauta) jimenezi Dugès, 1889 Epicauta (Epicauta) laevicornis Werner, 1973 Epicauta (Epicauta) leucocoma Champion, 1892 Epicauta (Epicauta) maculata (Say, 1823) Epicauta (Epicauta) major Pic, 1924 Epicauta (Epicauta) mexicana (Dugès, 1889) Epicauta (Epicauta) mixta Dugès, 1889 Epicauta (Epicauta) modesta (Haag-Rutenberg, 1880) Epicauta (Epicauta) nigerrima (Dugès, 1870) Epicauta (Epicauta) nigritarsis (LeConte, 1853) Epicauta (Epicauta) obesa (Chevrolat, 1834) Epicauta (Epicauta) occipitalis Werner, 1949 Epicauta (Epicauta) ocellata (Dugès, 1869) Epicauta (Epicauta) pardalis LeConte, 1866 Epicauta (Epicauta) pedalis LeConte, 1866 Epicauta (Epicauta) pensylvanica (De Geer, 1775) Epicauta (Epicauta) phoenix Werner, 1944 Epicauta (Epicauta) puncticollis Mannerheim, 1843 Epicauta (Epicauta) rileyi Horn, 1874 Epicauta (Epicauta) rufipennis (Chevrolat, 1834) Epicauta (Epicauta) senilis Werner, 1949 Epicauta (Epicauta) sericans LeConte, 1866 Epicauta (Epicauta) singularis Champion, 1892 Epicauta (Epicauta) straba Horn, 1891 Epicauta (Epicauta) subatra Dugès, 1889 Epicauta (Epicauta) subrubra Dugès, 1889 Epicauta (Epicauta) tarasca Pinto, 1991 Epicauta (Epicauta) temexa Adams et Selander, 1979 Epicauta (Epicauta) tenebrosa Werner, 1949 Epicauta (Epicauta) teresa Mathieu, 1983 Epicauta (Epicauta) tricostata (Werner, 1943) Epicauta (Epicauta) unilineata Champion, 1892 Epicauta (Epicauta) vitticollis (Haag-Rutenberg, 1880)

Subgénero Macrobasis LeConte, 1862

Epicauta (Macrobasis) alastor Skinner, 1904 Epicauta (Macrobasis) albida (Say, 1824) Epicauta (Macrobasis) apicalis Dugès, 1889 Epicauta (Macrobasis) arizonica Werner, 1944 Epicauta (Macrobasis) atricolor Champion, 1892 Epicauta (Macrobasis) atripilis Champion, 1892 Epicauta (Macrobasis) atrivittata (LeConte, 1854) Epicauta (Macrobasis) beckeri Dugès, 1889 Epicauta (Macrobasis) bipunctata Werner, 1958 Epicauta (Macrobasis) borrei (Dugès, 1881) Epicauta (Macrobasis) candezi (Haag-Rutenberg, 1880)
Epicauta (Macrobasis) cinereiventris Champion, 1892 Epicauta (Macrobasis) croceicincta (Dugès, 1881) Epicauta (Macrobasis) disparilis (Champion, 1892) Epicauta (Macrobasis) distorta (Champion, 1892)

Epicauta (Macrobasis) diversicornis (Haag-Rutenberg, 1880)

Epicauta (Macrobasis) evanescens Champion, 1892

Epicauta (Macrobasis) excors (Fall, 1909)

Epicauta (Macrobasis) forticornis (Haag-Rutenberg, 1880)

Epicauta (Macrobasis) funesta (Chevrolat, 1834)

Epicauta (Macrobasis) hirsutipubescens (Maydell, 1934)

Epicauta (Macrobasis) humeralis (Dugès, 1889)

Epicauta (Macrobasis) immaculata (Say, 1824)

Epicauta (Macrobasis) isthmica Werner, 1949

Epicauta (Macrobasis) labialis (Dugès, 1881)

Epicauta (Macrobasis) languida (Horn, 1895)

Epicauta (Macrobasis) lauta (Horn, 1885)

Epicauta (Macrobasis) lauta lauta (Horn, 1885)

Epicauta (Macrobasis) lauta rossi Werner, 1949

Epicauta (Macrobasis) leoni Dugès, 1889

Epicauta (Macrobasis) liebecki Werner, 1944

Epicauta (Macrobasis) linearis (LeConte, 1858)

Epicauta (Macrobasis) longicollis (LeConte, 1853)

Epicauta (Macrobasis) melanochroa Wellman, 1910

Epicauta (Macrobasis) nigritibialis Werner, 1958

Epicauta (Macrobasis) niveolineata (Haag-Rutenberg, 1880)

Epicauta (Macrobasis) ochrea (LeConte, 1853)

Epicauta (Macrobasis) pacifica Maydell, 1934

Epicauta (Macrobasis) polingi Werner, 1944

Epicauta (Macrobasis) prosopidis Werner, 1973

Epicauta (Macrobasis) punctum (Dugès, 1869)

Epicauta (Macrobasis) purpurea (Horn, 1885)

Epicauta (Macrobasis) segmenta (Say, 1824)

Epicauta (Macrobasis) selanderorum Werner, 1958

Epicauta (Macrobasis) stigmata (Dugès, 1869)

Epicauta (Macrobasis) sublineata (LeConte, 1854)

Epicauta (Macrobasis) tenella (LeConte, 1858)

Epicauta (Macrobasis) tenuemarginata Werner, 1958

Epicauta (Macrobasis) tenuicornis (Champion, 1892)

Epicauta (Macrobasis) tenuilineata (Horn, 1894)

Epicauta (Macrobasis) terminata (Dugès, 1869)

Epicauta (Macrobasis) texana Werner, 1944

Epicauta (Macrobasis) tripartita Champion, 1892

Epicauta (Macrobasis) triquetra Werner, 1958

Epicauta (Macrobasis) unicalcarata Champion, 1892

Epicauta (Macrobasis) uniforma Werner, 1944

Epicauta (Macrobasis) virgulata (LeConte, 1866)

Género Linsleya MacSwain, 1951

Subgénero Linsleya MacSwain, 1951

Linsleya (Linsleya) convexa (LeConte, 1853)

Linsleya (Linsleya) suavissima (Wellman, 1910)

Tribu Eupomphini LeConte, 1862

Género Cysteodemus LeConte, 1851

Cysteodemus armatus LeConte, 1851

Cysteodemus wislizeni LeConte, 1851

Género Eupompha LeConte, 1858

Eupompha decolorata (Horn, 1894) 
Eupompha elegans (LeConte, 1851)

Eupompha elegans elegans (LeConte, 1851)

Eupompha elegans perpulchra (Horn, 1870)

Eupompha fissiceps LeConte, 1858

Eupompha sulcifrons (Champion, 1892)

Eupompha terminalis Selander, 1957

Eupompha viridis (Horn, 1883)

Eupompha vizcaina Pinto, 1983

Eupompha wenzeli (Skinner, 1904)

Género Megetra LeConte, 1859

Megetra cancellata (Brandt et Erichson, 1832)

Megetra punctata Selander, 1965

Género Phodaga LeConte, 1858

Phodaga alticeps LeConte, 1858

Phodaga marmorata (Casey, 1891)

Género Pleuropasta Wellman, 1909

Pleuropasta mirabilis (Horn, 1870)

Pleuropasta reticulata Van Dyke, 1947

Género Tegrodera LeConte, 1851

Tegrodera aloga Skinner, 1903

Tegrodera erosa LeConte, 1851

Tegrodera erosa erosa LeConte, 1851

Tegrodera erosa extincta Beauregard, 1890

Tribu Lyttini LeConte, 1862

Género Lytta Fabricius, 1775

Subgénero Adicolytta Selander, 1960

Lytta (Adicolytta) cardinalis Chevrolat, 1833

Lytta (Adicolytta) erebea (Champion, 1892)

Lytta (Adicolytta) erythrothorax (Mendoza et Herrera, 1867)

Lytta (Adicolytta) eucera (Chevrolat, 1834)

Lytta (Adicolytta) mutilata (Horn, 1875)

Lytta (Adicolytta) peninsularis (Fall, 1901)

Lytta (Adicolytta) sanguinea Haag-Rutenberg, 1880

Subgénero Paralytta Selander, 1960

Lytta (Paralytta) augusti Haag-Rutenberg, 1880

Lytta (Paralytta) biguttata LeConte, 1853

Lytta (Paralytta) biguttata biguttata LeConte, 1853

Lytta (Paralytta) biguttata bivirgata (Dugès, 1881)

Lytta (Paralytta) biguttata pallens Selander, 1956

Lytta (Paralytta) bipuncticollis Haag-Rutenberg, 1880

Lytta (Paralytta) canelas Selander, 1960

Lytta (Paralytta) corallifera Haag-Rutenberg, 1880

Lytta (Paralytta) cribrata LeConte, 1854

Lytta (Paralytta) deserticola Horn, 1870

Lytta (Paralytta) ebenina (Dugès, 1877)

Lytta (Paralytta) incompta Pinto, 1985

Lytta (Paralytta) koltzei Haag-Rutenberg, 1880

Lytta (Paralytta) lecontei Heyden, 1890

Lytta (Paralytta) magister Horn, 1870

Lytta (Paralytta) margarita (Fall, 1901)

Lytta (Paralytta) melaena LeConte, 1858

Lytta (Paralytta) michoacanae (Champion, 1892)

Lytta (Paralytta) nitidicollis (LeConte, 1851)

Lytta (Paralytta) plumbea Haag-Rutenberg, 1880

Lytta (Paralytta) proteus Haag-Rutenberg, 1880

Lytta (Paralytta) quadrimaculata (Chevrolat, 1834)
Lytta (Paralytta) scitula (Champion, 1892)

Lytta (Paralytta) scituloides Selander, 1960

Lytta (Paralytta) sonorae Van Dyke, 1947

Lytta (Paralytta) variabilis (Dugès, 1869)

Lytta (Paralytta) vulnerata (LeConte, 1851)

Lytta (Paralytta) vulnerata vulnerata (LeConte, 1851)

Subgénero Poreospasta Horn, 1868

Lytta (Poreospasta) auriculata Horn, 1870

Lytta (Poreospasta) childii LeConte, 1857

Tribu Meloini Blanchard, 1845

Género Meloe Linnaeus, 1758

Subgénero Meloe Linnaeus, 1758

Meloe (Meloe) dugesi Champion, 1891

Meloe (Meloe) nebulosus Pinto et Selander, 1970

Meloe (Meloe) strigulosus Mannerheim, 1852

Subgénero Treiodous Dugès, 1869

Meloe (Treiodous) gracilicornis Champion, 1891

Meloe (Treiodous) laevis Leach, 1815

Género Spastonyx Selander, 1954

Tribu Pyrotini MacSwain, 1956

Género Denierota Kaszab, 1959

Género Pyrota Dejean, 1834

Pyrota akhurstiana Horn, 1891

Pyrota bilineata Horn, 1885

Pyrota concinna Casey, 1891

Pyrota deceptiva Selander, 1963

Pyrota decorata (Haag-Rutenberg, 1880)

Pyrota divirgata (Villada et Peñafiel, 1867)

Pyrota fasciata Selander, 1963

Pyrota hirticollis Champion, 1892

Pyrota insulata (LeConte, 1858)

Pyrota mariarum Champion, 1892

Pyrota mylabrina Chevrolat, 1834

Pyrota nigrovittata (Haag-Rutenberg, 1880)

Pyrota nobilis (Haag-Rutenberg, 1880)

Pyrota pacifica Selander, 1983

Pyrota palpalis Champion, 1893

Pyrota plagiata (Haag-Rutenberg, 1880)

Pyrota postica LeConte, 1866

Pyrota punctata Casey, 1891

Pyrota quadrinervata (Herrera et Mendoza, 1866)

Pyrota rugulipennis Champion, 1892

Pyrota tenuicostatis (Dugès, 1877)

Pyrota terrestris Selander, 1963

Pyrota trochanterica Horn, 1894

Pyrota trochanterica trochanterica Horn, 1894

Pyrota trochanterica werneri Selander, 1957

Subfamilia Nemognathinae Cockerell, 1910

Tribu Horiini Latreille, 1802

Género Cissites Latreille, 1804

Cissites auriculata (Champion, 1892) 
Cissites maculata (Swederus, 1787)

Tribu Nemognathini Lacordaire, 1859

Género Gnathium Kirby, 1818

Gnathium francilloni Kirby, 1818

Gnathium minimum (Say, 1824)

Gnathium nitidum Horn, 1870

Género Hornia Riley, 1877

Hornia mexicana Dugès, 1889

Hornia mexicana mexicana Dugès, 1889

Hornia mexicana blomi MacSwain, 1958

Género Nemognatha Illiger, 1807

Nemognatha (?) chrysomeloides (Linnaeus, 1763)

Nemognatha (?) ephippiata Champion, 1892

Nemognatha (?) foveifrons Champion, 1892

Subgénero Nemognatha Illiger, 1807

Nemognatha (Nemognatha) piazata (Fabricius, 1798)

Nemognatha (Nemognatha) piazata bicolor LeConte, 1853

Subgénero Meganemognatha Enns, 1956

Nemognatha (Meganemognatha) bridwelli Wellman, 1912

Nemognatha (Meganemognatha) explanata Enns, 1956

Nemognatha (Meganemognatha) lurida LeConte, 1853

Nemognatha (Meganemognatha) lurida lurida LeConte, 1853

Nemognatha (Meganemognatha) lurida apicalis LeConte, 1853

Nemognatha (Meganemognatha) lutea LeConte, 1853

Nemognatha (Meganemognatha) lutea lutea LeConte, 1853

Nemognatha (Meganemognatha) pallens LeConte, 1853

Subgénero Pauronemognatha Enns, 1956

Nemognatha (Pauronemognatha) brevicollis Champion, 1892

Nemognatha (Pauronemognatha) insularis Blaisdell, 1925

Nemognatha (Pauronemognatha) nigripennis LeConte, 1853

Nemognatha (Pauronemognatha) scutellaris LeConte, 1853

Nemognatha (Pauronemognatha) scutellaroides Wellman, 1910

Nemognatha (Pauronemognatha) selloa Selander, 1957

Subgénero Pronemognatha Enns, 1956

Nemognatha (Pronemognatha) cantharidis MacSwain, 1951

Nemognatha (Pronemognatha) flava (Dugès, 1889)

Nemognatha (Pronemognatha) selanderi Enns, 1956

Nemognatha (Pronemognatha) zonitoides Dugès, 1874

Género Pseudozonitis Dillon, 1952

Pseudozonitis martini (Fall, 1907)

Pseudozonitis megalops (Champion, 1892)

Pseudozonitis vaurieae Enns, 1956

Género Rhyphonemognatha Enns, 1956

Rhyphonemognata flavicollis (Dugès, 1881)

Rhyphonemognata rufa (LeConte, 1854)

Rhyphonemognata sanguinicollis (Champion, 1892)

Rhyphonemognata tenebrosa (Champion, 1892)

Género Zonitis Fabricius, 1775

Zonitis (?) nigromaculata Dugès, 1889

Zonitis (?) xanthochroa Wellman, 1910
Subgénero Neozonitis Enns, 1956

Zonitis (Neozonitis) atripennis (Say, 1824)

Zonitis (Neozonitis) florhi Dugès, 1889

Zonitis (Neozonitis) minutissima Pinto, 2001

Zonitis (Neozonitis) ploribunda Wellman, 1910

Subgénero Parazonitis Enns, 1956

Zonitis (Parazonitis) dugesi Selander, 1954

Zonitis (Parazonitis) sayi Wickham, 1905

Zonitis (Parazonitis) tarasca (Dugès, 1889)

Zonitis (Parazonitis) tarasca tarasca (Dugès, 1889)

Zonitis (Parazonitis) tarasca borealis Enns, 1956

Subfamilia Tetraonycinae Böving et Craighead, 1930

Tribu Tetraonycini Böving et Craighead, 1930

Género Tetraonyx Latreille, 1805

Subgénero Tetraonyx Latreille, 1805

Tetraonyx (Tetraonyx) angulicollis Haag-Rutenberg, 1879

Tetraonyx (Tetraonyx) batesi Haag-Rutenberg, 1879

Tetraonyx (Tetraonyx) bimaculatus (Klug, 1825)

Tetraonyx (Tetraonyx) bipartitus Haag-Rutenberg, 1879

Tetraonyx (Tetraonyx) decipiens Haag-Rutenberg, 1879

Tetraonyx (Tetraonyx) dubiosus Horn, 1894

Tetraonyx (Tetraonyx) femoralis Dugès, 1869

Tetraonyx (Tetraonyx) frontalis Chevrolat, 1833

Tetraonyx (Tetraonyx) fulvus LeConte, 1853

Tetraonyx (Tetraonyx) maculatus Laporte de Castelnau, 1840

Tetraonyx (Tetraonyx) octomaculatus Latreille, 1805

Tetraonyx (Tetraonyx) sallei Haag-Rutenberg, 1879

Tetraonyx (Tetraonyx) sexguttatus (Olivier, 1795)

\section{Catálogo taxonómico}

Familia Meloidae Gyllenhal, 1810

Melooides Gyllenhal, 1810: 481 (*)

Subfamilia Meloinae Ganglbauer, 1907

Meloinae Ganglbauer, 1907: 106 (*)

CONTENIDO Y DISTRIBUCIÓN: La subfamilia Meloinae está distribuida por la mayor parte del Planeta. La fauna americana, representada por 30 géneros, incluye representantes de las tribus Epicautini, Eupomphini, Lyttini, Meloini y Pyrotini (Pinto \& Bologna, 1999). En México están representados 13 géneros distribuidos en esas mismas tribus.

Tribu Epicautini Denier, 1935

Epicautini Denier, 1935a: $151(*)$

GÉNERO TIPO: Epicauta Dejean, 1834

CONTENIDO Y DISTRIBUCIÓN: En América la tribu está representada por dos géneros, Epicauta y Linsleya, ambos presentes en México.

Los últimos estudios filogenéticos (Bologna \& Pinto, 2001) parecen apoyar la inclusión de Linsleya dentro de la tribu Epicautini, aunque dada la discusión existente al 
respecto (Selander, 1955a; Kaszab, 1963b; Selander, 1964b; Gupta, 1965; Selander, 1982a; Pinto \& Bologna, 1999), la falta de resolución en algunos nodos críticos de la hipótesis de Bologna \& Pinto (2001) y la existencia de homoplasia en muchos de los caracteres, consideramos que el tema require más estudio.

Género Epicauta Dejean, 1834

Epicauta Dejean, 1834: 224

ESPECIE TIPO: Meloe erythrocephala Pallas, 1776, por designación subsecuente de Werner (1945).

CONTENIDO Y DISTRIBUCIÓN: El género incluye unas 375 especies distribuidas por casi todo el Planeta; de éstas unas 173 especies viven en América del Norte y otras 100 en América del Sur (Pinto \& Bologna, 1999). Aunque el género ha sufrido subdivisiones diversas, actualmente se reconocen dos subgéneros, Epicauta y Macrobasis, el primero de amplia distribución mundial, el segundo casi exclusivo de América del Norte (Pinto, 1991). Ambos subgéneros están representados en México.

Zaragoza-Caballero \& Velasco-de León (2003) han descrito una especie fósil de México que no ha sido asignada a ningún subgénero concreto:

Epicauta sanctoruensis Zaragoza-Caballero \& Velascode León, 2003

Epicauta sancturoensis Zaragoza-Caballero \& Velasco-de León, 2003: $156(*)$

TERRA TYPICA: "Sanctuorum, en la Formación Atotonilco El Grande, Estado de Hidalgo, México" "Plioceno". Holotipo n CFZ-ST 28, en la colección de Paleontología de la Facultad de Estudios Superiores Zaragoza de la Universidad Nacional Autónoma de México (fide Zaragoza-Caballero \& Velasco-de León, 2003).

DISTRIBUCIÓN: Especie descrita con material de hace unos $4 \mathrm{Ma}$, procedente del fondo de un lago del Plioceno del Estado de Hidalgo (Zaragoza-Caballero \& Velascode León, 2003).

\section{Subgénero Epicauta Dejean, 1834}

Epicauta Dejean, 1834: 224

ESPECIE TIPO: Meloe erythrocephala Pallas, 1776, por designación subsecuente de Werner (1945)

Causima Dejean, 1834: 226

ESPECIE TIPO: Lytta vidua Klug, 1825, por monotipia (ver Dejean, 1837).

Henous Haldeman, 1852: 377 (*)

ESPECIE TIPO: Henous techanus Haldeman, 1852 (= Meloe confertus Say, 1824), por monotipia.

Isopentra Mulsant et Rey, 1858: 106 (*)

ESPECIE TIPO: Lytta megalocephala Gebler, 1817, por designación subsecuente de Wellman (1910).

Pleuropompha LeConte, 1862: 273 (*)

ESPECIE TIPO: Lytta costata LeConte, 1854, por monotipia.

Nomaspis LeConte, 1866: 156 (*)

ESPECIE TIPO: Meloe parvus Haldeman, 1852, por monotipia.

Anomalonyx Denier, 1935a: 161 (non Anomalonyx Weise, 1903, Chrysomelidae, nec Moser, 1921, Scarabaeidae) (*)

ESPECIE TIPO: Lytta fumosa Germar, 1824, por designación original
Anomalonychus Saylor, 1940: 46 (nombre de substitución para Anomalonyx Denier, 1935) (*)

Maculicauta Dillon, 1952: 416 (*)

ESPECIE TIPO: Epicauta stuarti LeConte, 1868, por designación original.

COMENTARIOS TAXONÓMICOS: Pinto (1984b) establece la sinonimia de Pleuropompha y Anomalonychus con Epicauta. Muchos de los nombres genéricos incluidos en la sinonimia de Epicauta podrían corresponder a géneros o subgéneros válidos cuando se revise la tribu Epicautini en su conjunto. Los nombres Henons y Epicauata publicados por Dugès (1869a: 102 y 1889e: 81, respectivamente) son meros errores tipográficos.

CONTENIDO Y DISTRIBUCIÓN: El subgénero incluye unas 300 especies distribuidas por casi todo el Planeta, de las cuales, unas 100, viven en América del Norte (Pinto \& Bologna, 1999). Pinto (1991) agrupa a estas especies norteamericanas en 18 grupos de especies, la mayoría de ellos representados en México.

El total de especies registradas en México asciende a 120, más al menos una sin describir (Pinto, 1991: ver comentarios sobre E. occipitalis). Además de éstas, existen muchas otras especies bien representadas en California, Arizona, Nuevo México y Texas (EEUU), que posiblemente se encuentren también en México y cuya ausencia se debe sin duda a falta de prospección. Epicauta aspera Werner, 1944, una de esas especies de presencia probable en México, ha sido localizada muy cerca de la frontera en Nuevo México y Texas y existe un ejemplar con etiqueta de "Guadalajara, Mex." en la Canadian National Collection (Ottawa, Canadá), aunque Pinto (1991) considera que este ejemplar está mal etiquetado y por lo tanto, la especie de momento no puede considerarse presente en México. Champion (1892) y Blackwelder (1945) señalan la presencia en México de Epicauta cinerea (Forster, 1771), una especie que se distribuye por la región oriental del Canadá y de los EEUU, hasta Texas. Pinto (1991) indica que en numerosas obras existen confusiones en la identificación de E. cinerea y no hace mención de registro mexicano alguno para esta especie. Pinto (1991), además, indica que las citas de Champion (1892) de E. cinerea corresponden a E. major (sensu Pinto, 1991) y aunque no hace mención explícita de ello consideramos que las de Blackwelder (1945) también. Por lo tanto, y aunque la presencia de E. cinerea es probable en el norte de México, la especie no ha sido incluida en este catálogo.

\section{Epicauta (Epicauta) abadona Skinner, 1904}

Epicauta abadona Skinner, 1904: 217 (*)

TERRA TYPICA: "Phoenix and Tucson, Arizona". Holotipo no 8068, de Phoenix, en la colección de la Academy of Natural Sciences of Philadelphia (EEUU) (fide Werner, 1945; Adams \& Selander, 1979; Pinto, 1991). Skinner (1904) indicó que el tipo fue depositado en la colección de la American Entomological Society.

Epicauta mutchleri Maydell, 1934: 331 (*) 
TERRA TYPICA: “...Phoenix, Arizona...”. Holotipo, en la colección del American Museum of Natural History (EEUU) (fide Maydell, 1934; Werner, 1945; Adams \& Selander, 1979; Pinto, 1991).

COMENTARIOS TAXONÓMICOS: El error tipográfico Epicauta abandona se deslizó en la obra de Maydell (1934: 331). Especie incluida en el grupo de E. vittata por Adams \& Selander (1979), Agafitei \& Selander (1980) y Pinto (1991).

DISTRIBUCIÓN GEOGRÁFICA: Especie con una distribución restringida al norte de México y suroeste de los EEUU (Pinto, 1991). Citada en los estados de Sinaloa y Sonora.

MÉXICO: Sinaloa: Concordia (Adams \& Selander, 1979); Culiacán (Adams \& Selander, 1979; Agafitei \& Selander, 1980); 57 mi. al N de Culiacán (Adams \& Selander, 1979); Los Mochis (Werner et al., 1966; Adams \& Selander, 1979); Mazatlán (Werner, 1945; Werner et al., 1966; Adams \& Selander, 1979); 20 mi. al N de Pericos (Adams \& Selander, 1979); Puente de que Malo [sic] (Adams \& Selander, 1979); Venedio [sic] (posiblemente Venadillo, ver Selander, 1960a) (Werner et al., 1966; Adams \& Selander, 1979). Sonora: Álamos (Adams \& Selander, 1979); $20 \mathrm{mi}$. al SE de Agua Prieta (Adams \& Selander, 1979); Ciudad Obregón (Werner et al., 1966; Adams \& Selander, 1979); Hermosillo (Adams \& Selander, 1979); 40 mi. al N de Hermosillo (Adams \& Selander, 1979).

\section{Epicauta (Epicauta) abeona Pinto, 1980}

Epicauta abeona Pinto, 1980: 69 (*)

TERRA TYPICA: "near Ixtlan del Rio, Nayarit, Mexico". Holotipo en la colección del Los Angeles County Museum (EEUU) (fide Pinto, 1980)

COMENTARIOS TAXONÓMICOS: Incluida en el grupo de E. maculata por Pinto (1980, 1991).

DISTRIBUCIÓN GEOGRÁFICA: Endemismo mexicano (Pinto, 1980, 1991). Citado en los estados de Aguascalientes, Distrito Federal, Durango, Jalisco, México, Michoacán y Nayarit.

MÉXICO: Aguascalientes: $10 \mathrm{mi}$. al E de Aguascalientes (Pinto, 1980); 15 mi. al E de Aguascalientes (Pinto, 1980). Distrito Federal: Guadalupe (Pinto, 1980); Ciudad de México (Pinto, 1980). Durango: Donato Guerra (Pinto, 1980); Durango (Pinto, 1980); 6 mi. al E de Durango (Pinto, 1980). Jalisco: cerca de Ameca (Pinto, 1980); cerca de Guadalajara (Pinto, 1980); cerca de Pegueros (Pinto, 1980); 9 mi. al O de Tepatitlán (Pinto, 1980); al N de Tepatitlán (Pinto, 1980); 2 mi. al S de Tlaquepaque (Pinto, 1980); Tonala (Pinto, 1980). México: San Juan Teotihuacán (Pinto, 1980). Michoacán: 9 $\mathrm{km}$ al NE de Pátzcuaro (Pinto, 1991); 14 mi. al E de Tuxpan (Pinto, 1991). Nayarit: Compostela (Pinto, 1980); cerca de Ixtlán del Río (Pinto, 1980); 14 mi. al S de Tepic (Pinto, 1980); Tepic (Pinto, 1980).

\section{Epicauta (Epicauta) afoveata Werner, 1949}

Epicauta afoveata Werner, 1949b: 103 (*)

TERRA TYPICA: "Borrego, San Diego Co., California". Holotipo n 28220 en la colección del Museum of Comparative Zoology (Harvard University, Cambridge, EEUU) (fide Werner, 1949b; Pinto, 1991).
COMENTARIOS TAXONÓMICOS: Especie incluida en el grupo de E. caviceps por Werner (1955) y Pinto (1991). Pinto (1991) indica que los ejemplares de Baja California difieren considerablemente del resto, aunque no propone ningún cambio taxonómico al respecto.

DisTRIBUCIÓN GEOGRÁFICA: Especie distribuida por el noroeste de México y el suroeste de los EEUU (Pinto, 1991). En México se ha citado únicamente en la Península de Baja California.

MÉxICo: Baja California: $7.8 \mathrm{mi}$. al SE de Cataviña (Pinto, 1991); Isla Cedros, cerca del pico Cerro de Cedros (Pinto, 1991).

\section{Epicauta (Epicauta) albolineata (Dugès, 1877) \\ Cantharis albolineata Dugès, 1877: 64 (*)}

TERRA TYPICA: "Guanajuato" en la descripción original, aunque posiblemente figure "Tupátaro" en la etiqueta (fide Pinto, 1991). Holotipo $\mathrm{n}^{\circ} 822$ (IBUNAM 011), en la colección del Instituto de Biología de la Universidad Nacional Autónoma de México (DF) (fide Vázquez \& Zaragoza, 1979; Pinto, 1991).

Epicauta albolineata (Dugès, 1877): Dugès, 1886a: $582(*)$

Epicauta duplicata Casey, 1891: 172 (*)

TERRA TYPICA: "Arizona (Fort Apache)". Holotipo en la colección del United States National Museum of Natural History (Washington D.C.), no 49210 (fide Pinto, 1991).

Epicauta costaricensis Kaszab, 1960: 284 (*)

TERRA TYPICA: “Costa Rica...”. Holotipo en la colección del Magyar Természettudományi Múzeum de Budapest (Hungría) (fide Kaszab, 1960; Pinto, 1991).

Comentarios taxonómicos: Pinto (1991) establece la sinonimia de E. costaricensis con E. albolineata. Dugès (1877) indica la existencia del nombre no disponible Epicauta albolineata Sturm, 1843: 175. Especie incluida en el grupo de E. albolineata por Pinto (1991).

DisTRIBUCIÓN GEOGRÁFICA: Especie con una distribución muy amplia, desde Costa Rica hasta el sur de los EEUU, con registros en México, Honduras (Lancatilla) y Guatemala (Pinto, 1991). En México se ha citado en los estados de Aguascalientes, Colima, Guanajuato, Guerrero, Jalisco, México, Michoacán, Morelos, Nayarit, Oaxaca, Puebla, Querétaro, San Luis Potosí y Sonora. Pinto (1991) no considera las citas de San Juan del Río, Mochitlán, Izúcar de Matamoros y Oaxaca de Champion (1892).

MÉxICO: Aguascalientes: $10 \mathrm{mi}$. al E de El Retono [Retorno] (Pinto, 1991). Colima: (Pinto, 1991). Guanajuato: Guanajuato (Dugès, 1877, 1889e; Champion, 1892; ZaragozaCaballero, 1999); Tupátaro (Dugès, 1889e; Champion, 1892; Vázquez \& Zaragoza, 1979; Pinto, 1991; Zaragoza-Caballero, 1999). Guerrero: Chilpancingo (Champion, 1892; Pinto, 1991); Iguala (Champion, 1892; Pinto, 1991); Mochitlán (Champion, 1892); Omilteme (Pinto, 1991). Jalisco: Ameca (Pinto, 1991); Arenal (Pinto, 1991); Degollado (Pinto, 1991); Guadalajara (Pinto, 1991); Pacana (Pinto, 1991); al N de Tepatitlán (Pinto, 1991); 9 mi. O Tepatitlán (Pinto, 1991); Tequila (Pinto, 1991). México: Temascaltepec (Pinto, 1991). Michoacán: $35 \mathrm{~km}$ al E de Jiquilpan (Pinto, 1991); Morelia (Zaragoza-Caballero, 1999); Quiroga (Dugès, 1889e; Zaragoza-Caballero, 1999). Morelos: Alpuyeca (Pinto, 1991); 
Cuernavaca (Champion, 1892; Pinto, 1991); Tres Marías (Pinto, 1991). Nayarit: Compostela (Pinto, 1991); Ixtlán del Río (Pinto, 1991); La Mesa de Nayar (Pinto, 1991). Oaxaca: Istmo de Tehuantepec (Pinto, 1991); Oaxaca (Champion, 1892). Puebla: Izúcar de Matamoros (Champion, 1892). Querétaro: San Juan del Río (Champion, 1892). San Luis Potosí: Tamazunchale (Pinto, 1991). Sonora: Guirocoba (Pinto, 1991).

\section{Epicauta (Epicauta) andersoni Werner, 1944}

Epicauta andersoni Werner, 1944: 66 (*)

TeRra TYPICA: "Gallo Springs, New Mexico". Holotipo no 56883 en la colección del United States National Museum of Natural History (Washington D.C.). Paratipos de New Mexico, Texas y Kansas (Werner, 1944).

COMENTARIOS TAXONÓMICOS: Especie incluida en el grupo de E. maculata por Pinto $(1980,1991)$.

DISTRIBUCIÓN GEOGRÁFICA: Especie distribuida por el extremo septentrional de México y el centro-suroeste de los EEUU (Pinto, 1980, 1991). En México se ha citado únicamente en Chihuahua.

MÉxICo: Chihuahua: Basuchil (Pinto, 1980); Ojinaga (Pinto, 1980); San José Babícora (Vaurie, 1950; Pinto, 1980); Santa Bárbara (Pinto, 1980); Delicias (Vaurie, 1950).

\section{Epicauta (Epicauta) apache Pinto, 1980}

Epicauta apache Pinto, 1980: 62 (*)

TERRA TYPICA: " 4.8 mi. southeast of Willcox, Cochise County, Arizona". Holotipo, en la colección de la California Academy of Sciences (San Francisco, EEUU) (fide Pinto, 1980).

COMENTARIOS TAXONÓMICOS: Incluida en el grupo de E. maculata por Pinto (1980, 1991).

DistribuCión GEOGRÁFICA: Conocida del noroeste de México y del centro y sur de los EEUU (Pinto, 1991). Citado en los estados de Chihuahua y Sonora. Citada por Werner (1945; 1949b), Dillon (1952), Werner et al. (1966), Pinto (1975b) y Arnold (1976) con el nombre de E. maculata (Pinto, 1980, 1991).

MÉxICo: Chihuahua: $7 \mathrm{~km}$ al N de Jiménez (Pinto, 1980); 36 mi. al S de Parrita (Pinto, 1980). Sonora: sin más precisión (Pinto, 1980).

Epicauta (Epicauta) atrata (Fabricius, 1775)

Lytta atrata Fabricius, 1775: 260 (*)

TERRA TYPICA: "America" originalmente; "North America" tras designación de lectotipo por Werner (1945), en la colección del Hunterian College (Glasgow University), que corresponde al ejemplar figurado por Staig (1940: lám. 55).

Cantharis atrata (Fabricius, 1775): Olivier, 1790: 280

Meloe trichrus Pallas, 1798: $100(*)$

TERRA TYPICA: “...Noveboracensia Americes...”. Tipos no localizados (fide Pinto, 1991).

Lytta coracina Illiger, 1804: 171 (nombre substituto para L. atrata Fabricius, innecesario) $(*)$

Lytta trichrus (Pallas, 1798): Schoenherr, 1817: 28

Cantharis trichrus (Pallas, 1798): Fischer, 1827: 23

Lytta convolvuli Melsheimer, 1846: 53 (*)

TERRA TYPICA: No indicada en la descripción original, aunque Beauregard (1890) señala Pennsylvania. Tipos no localizados (fide Pinto, 1991).
Epicauta convolvuli (Melsheimer, 1846): LeConte, 1866: 157 (*)

Epicauta trichrus (Pallas, 1798): Horn, 1891: $43\left({ }^{*}\right)$

Epicauta atrata (Fabricius, 1775): Werner, 1945: 470 (*)

COMENTARIOS TAXONÓMICOS: Especie incluida en el grupo de E. sericans por Pinto (1991). LeConte (1853) reconoce a nivel de especie a E. convolvuli, pero Pinto (1991), al igual que otros autores anteriores, señala que a la variación en el color de la cabeza o de la pilosidad no puede atribuirsele carácter diagnóstico. Borchmann (1917) incluye a E. atrata y E. coracina como sinónimos de E. pensylvanica, mientras que considera a E. trichrus (incluida $E$. convolvuli) como una especie independiente. Linnaeus (1763) indica erróneamente como localidad de L. atrata: "Barbaria". Wellman (1910c: 24) indica por error el nombre Epicauta trichura y Gibson (1912: 87) el de Epicauta trichus.

DisTRIBUCIÓN GEOGRÁFICA: Especie ampliamente distribuida por el noreste de México y el este de los EEUU, con algunos registros en el sur del Canadá (Pinto, 1991). En México ha sido citada en los estados de Nuevo León, San Luis Potosí y Tamaulipas. Existe un ejemplar del Río Metlac (cerca de Fortín de las Flores), Veracruz, en la colección del Essig Museum, University of California (Berkeley, EEUU), que Pinto (1991) considera de procedencia dudosa.

México: Nuevo León: Cascada Cola de Caballo (Pinto, 1991); Linares (Pinto, 1991); $10 \mathrm{mi}$. al O de Linares (Pinto, 1991); 16 mi. al S de Linares (Pinto, 1991); 3 mi. al S de Montemorelos (Pinto, 1991); $26 \mathrm{~km}$ al SSE de Montemorelos (Pinto, 1991); Rincón de la Sierra (Pinto, 1991); 2 mi. al SE de Salinas Victoria (Pinto, 1991). San Luis Potosí: 5 mi. al SE de Ciudad del Maíz (Pinto, 1991). Tamaulipas: $9 \mathrm{mi}$. al S de Villagran (Pinto, 1991).

Epicauta (Epicauta) basimacula (Haag-Rutenberg, 1880)

\section{Lytta basimacula Haag-Rutenberg, 1880: 48 (*)}

TERRA TYPICA: "Mexico". Lectotipo designado por Pinto (1982) en la colección del Zoologische Staatssammlung (Munich). La serie típica consta de dos ejemplares más (Pinto, 1982)

Cantharis rufescens Dugès, 1881: 142 (*)

TERra typiCa: "Cotija". Tipo aparentemente perdido (fide Pinto, 1991).

Epicauta rufescens (Dugès, 1881): Dugès, 1886a: 582 (*)

Epicauta basimacula (Haag-Rutenberg, 1880): Beauregard, 1890: 502 (*) Cantharis basimacula (Haag-Rutenberg, 1880): Champion, 1899: 172 (*)

COMENTARIOS TAXONÓMICOS: Incluida en el grupo de E. cupraeola por Pinto (1991). Beauregard (1890) considera a $C$. rufescens especie válida.

DisTRIBUCIÓN GEOGRÁFICA: Extendida desde el centro de México hasta el extremo sudoccidental de Guatemala (Pinto, 1991). En México se ha citado en los estados de Chiapas, Colima, Guerrero, Jalisco, México, Michoacán, Morelos, Nayarit, Oaxaca y Veracruz.

MÉxICo: Chiapas: Ciudad Cuauhtémoc (Pinto, 1991). Colima: Vulkan Colima (Nevado de Colima) (Pinto, 1991). Guerrero: Chilpancingo (Pinto, 1991); Iguala (Dugès, 1881; Dugès, 1889e sub C. rufescens; Champion, 1892; Pinto, 1991); 
Mexcala (Pinto, 1991). Jalisco: 5 mi. al $\mathrm{S}$ de Atenquique (Pinto, 1991); Guadalajara (Pinto, 1991); $18 \mathrm{mi}$. al S de Guadalajara (Pinto, 1991); $7.5 \mathrm{~km}$ al NE de Juchitlán (Pinto, 1991); La Venta (Pinto, 1991); Tequila (Pinto, 1991). México: Bejucos (Pinto, 1991); Tejupilco (Pinto, 1991); Teotihuacán (Pinto, 1991). Michoacán: Capirio (Pinto, 1991); Cotija (Dugès, 1881 sub C. rufescens; Dugès, $1889 \mathrm{e}$ sub C. rufescens; Champion, 1892); Nueva Italia (Pinto, 1991). Morelos: Alpuyeca (Pinto, 1991); Cuernavaca (Pinto, 1991); $18 \mathrm{mi}$. al SE de Cuernavaca (Pinto, 1991); Hermosa (Pinto, 1991). Nayarit: Arroyo Santiago, cerca de Jesús María (Pinto, 1991); Jesús María (Pinto, 1991); San Juan Peyotán (Pinto, 1991); Ixtlán del Río (Pinto, 1991). Oaxaca: El Camarón (Pinto, 1991); Jamiltepec (Pinto, 1991); Tehuantepec (Champion, 1892). Veracruz: Xalapa (Pinto, 1991).

\section{Epicauta (Epicauta) bispinosa Werner, 1949}

Epicauta bispinosa Werner, 1949b: 95 (*)

TERRA TYPICA: "10 mi. E of Sonoita, Sta. Cruz Co., Arizona, alt. 4800 ft.". Holotipo en la colección del Museum of Comparative Zoology (Harvard University, Cambridge, EEUU) (fide Werner, 1949b), pero no tiene el no 28219 indicado por Werner (fide Pinto, 1991).

COMENTARIOS TAXONÓMICOS: Especie incluida en el grupo de E. bispinosa por Pinto $(1975 b ; 1991)$ y en el de E. maculata por Selander (1981).

DisTRIBUCIÓN GEOGRÁFICA: Especie distribuida por el norte de México y el sur de los EEUU (Pinto, 1991). En México se extiende desde Durango hasta la frontera con EEUU (Pinto, 1975b).

MÉXICO: Durango: 2 mi. al S de Zarca (Pinto, 1975b).

\section{Epicauta (Epicauta) brunnea Werner, 1944}

Epicauta brunnea Werner, 1944: 67 (*)

TERRA TYPICA: “Alpine, Texas, 6000 ft.”. Holotipo n ${ }^{\circ} 26604$ en la colección del Museum of Comparative Zoology (Harvard University, Cambridge, EEUU).

Epicauta innomina Dillon, 1952: 401 (*)

TERRA TYPICA: “Tyler, Texas...”. Holotipo no 7164 en la colección de la Texas Agricultural and Mechanical University (EEUU) (fide Pinto, 1991).

COMENTARIOS TAXONÓMICOS: Incluida en el grupo de E. corvina por Pinto (1991). Pinto (1982) establece la sinonimia de E. innomina con E. brunnea.

DisTRIBUCIÓN GEOGRÁFICA: Especie distribuida por el norte de México y el sur de los EEUU (Pinto, 1991). En México se ha citado en los estados de Chihuahua, Sinaloa y Sonora.

MÉXICo: Chihuahua: 3 mi. al N de Chihuahua (Pinto, 1991); 46 mi. al N de Chihuahua (Pinto, 1991); Salaices (Werner et al., 1966; Pinto, 1991). Sinaloa: 46 mi. al E de Mazatlán (Pinto, 1991). Sonora: $12 \mathrm{~km}$ al S de Magdalena (Pinto, 1991); San Miguel (Pinto, 1991); Santa Ana (Pinto, 1991).

Epicauta (Epicauta) callosa LeConte, 1866

Epicauta callosa LeConte, 1866: 158 (*)

TERRA TYPICA: "Texas..., Nebraska...," originalmente; "Nebraska" tras designación de lectotipo. Lectotipo $\mathrm{n}^{\circ} 4996$, designado por Werner (1945) en la colección del Museum of Comparative Zoology (Harvard University, Cambridge, EEUU).
Epicauta pseudocallosa Dillon, 1952: 387 (*)

TERRA TYPICA: "McLennan Co., Texas". Holotipo en la colección de la Texas Agricultural and Mechanical University (EEUU) (fide Pinto, 1991).

Comentarios taxonómicos: Especie incluida en el grupo de E. sericans por Pinto (1991). Pinto (1991) sinonimiza E. pseudocallosa con E. callosa.

DisTRIBUCIÓN GEOGRÁFICA: Especie ampliamente distribuida por el norte de México y el centro-este de los EEUU (Pinto, 1991). En México ha sido citada en los estados de Chihuahua, Coahuila, Nuevo León y Tamaulipas.

MÉXICo: Chihuahua: Casa Grande (Pinto, 1991); Delicias (Vaurie, 1950; Pinto, 1991); 10 mi. al S de Delicias (Vaurie, 1950; Pinto, 1991); 10 mi. al N de Jiménez (Pinto, 1991); $18 \mathrm{mi}$. al O de Jiménez (Pinto, 1991); Juárez (Pinto, 1991); 3 mi. al NO de Meoqui (Pinto, 1991); La Cruz (Vaurie, 1950); La Mesa (Pinto, 1991). Coahuila: $10 \mathrm{mi}$. al S de Sabinas (Pinto, 1991). Nuevo León: Apodaca (Pinto, 1991); 5 mi. al E en la carretera a Reynosa (Pinto, 1991); Escobedo (Pinto, 1991); 16 mi. al N de La Gloria (Pinto, 1991); Linares (Pinto, 1991); 14 mi. al N de Linares (Pinto, 1991); 10 mi. al O de Linares (Pinto, 1991); Mesa de Chipinque (Pinto, 1991); Montemorelos (Pinto, 1991); $8 \mathrm{~km}$ al NE de Montemorelos (Pinto, 1991); 9 mi. al N de Montemorelos (Pinto, 1991); 20 mi. al S de Monterrey (Pinto, 1991); Río San Juan (Pinto, 1991). Tamaulipas: 62 mi. al NE de Ciudad Victoria (Pinto, 1991); $1 \mathrm{~km}$ al N de Encinal (Pinto, 1991); $63 \mathrm{mi}$. al N de San Fernando (Pinto, 1991); 9 mi. al S de Villagran (Pinto, 1991).

Epicauta (Epicauta) candidata Champion, 1892

Epicauta candidata Champion, 1892: $426\left({ }^{*}\right)$

TERra TYPICA: "MeXICO, Villa Lerdo in Durango". Holotipo, en la colección del British Museum of Natural History (Londres), al parecer muy dañado (fide Pinto, 1991).

Cantharis candidata (Champion, 1892): Champion, 1899: 174 (*)

COMENTARIOS TAXONÓMICOS: Incluida en el grupo de E. candidata por Pinto (1991).

DisTRIBUCIÓN GEOGRÁFICA: Extendida desde el norte de México hasta el sur de los EEUU (Pinto, 1991). En México se ha citado en los estados de Chihuahua, Coahuila, Durango y Zacatecas.

MÉxICo: Chihuahua: Ciudad Juárez (Pinto, 1991). Coahuila: Torreón (Pinto, 1991). Durango: Ciudad Lerdo (Champion, 1892; Vaurie, 1950; Pinto, 1991). Zacatecas: Zacatecas (Pinto, 1991).

\section{Epicauta (Epicauta) cardui (Dugès, 1889)}

Henous cardui Dugès, $1889 \mathrm{~d}$ : $36(*)$

TERRA TYPICA: "Mazamithl, Uruapan (Michoacán) Toluca" originalmente; "Toluca, México", tras designación de neotipo en la colección del United States National Museum of Natural History (Washington D.C.) (Pinto, 1991). Según Pinto (1991), el ejemplar ${ }^{\circ}$ 782 de la colección del Instituto de Biología de la Universidad Nacional Autónoma de México, indicado como tipo por Vázquez \& Zaragoza (1979), pertenece al subgénero Macrobasis y por lo tanto no puede ser $E$. (E.) cardui.

Epicauta cardui (Dugès, 1889): Pinto, 1991: 174 (*)

COMEnTARIos taxonómicos: Especie incluida en el grupo de E. cinerea por Pinto (1991). Pinto (1991) indi- 
ca que el color de la pilosidad en esta especie es muy variable y que esta variación parece tener una cierta correspondencia geográfica.

DisTRIBUCIÓN GEOGRÁfICA: Endemismo mexicano, distribuido desde Oaxaca en el sur hasta Durango en el norte (Pinto, 1991). Se ha citado en los estados de Aguascalientes, Colima, Distrito Federal, Durango, Guanajuato, Jalisco, México, Michoacán, Morelos, Oaxaca y Querétaro. Según Dugès (1889d), Champion (1891) y Borchmann (1917) las citas de Dugès (1869a) de $H$. confertus corresponden a $E$. cardui.

MÉXICO: Aguascalientes: $8 \mathrm{mi}$. al E de Aguascalientes (Pinto, 1991); 15 mi. al E de Aguascalientes (Pinto, 1991); 8 mi. al N de Aguascalientes (Pinto, 1991). Colima: $10 \mathrm{mi}$. O Colima (Pinto, 1991). Distrito Federal: Guadalupe (Pinto, 1991). Durango: Sierra de Durango (Pinto, 1991). Guanajuato: Celaya (Pinto, 1991). Jalisco: Ciénega de Mata (Pinto, 1991); Guadalajara (Pinto, 1991); 3 mi. al NE de Mazamitla (Pinto, 1991); Mazamitla (Dugès, 1889d; Champion, 1891); $11 \mathrm{mi}$. al S de Ojuelos (Pinto, 1991) México: $3 \mathrm{mi}$. al N de Atlacomulco (Pinto, 1991); $15.2 \mathrm{mi}$. al N de San Jerónimo (Pinto, 1991); Toluca (Dugès, 1889d; Champion, 1891; Pinto, 1991; Zaragoza-Caballero, 1999); Tultenango (Pinto, 1991). Michoacán: Carapan (Pinto, 1991); cerca de Comanja (Pinto, 1991); $10 \mathrm{mi}$. O Jiquilpan (Pinto, 1991); Pátzcuaro (Pinto, 1991); 5.5 mi. al E de Pátzcuaro (Pinto, 1991); $9 \mathrm{~km}$ al NE de Pátzcuaro (Pinto, 1991); $14 \mathrm{mi}$. O Pátzcuaro (Pinto, 1991); 16 mi. al SE de Morelia (Pinto, 1991); Sierra de Meayamitle (Dugès, 1869a sub H. conferta); cerca de Tzintzuntzan (Pinto, 1991); 4 mi. al E de Uruápan (Pinto, 1991); Uruápan (Dugès, 1889d; Champion, 1891; Zaragoza-Caballero, 1999). Morelos: Yautepec (Pinto, 1991). Oaxaca: Oaxaca (Champion, 1891; Pinto, 1991). Querétaro: 3 mi. al O de Querétaro (Pinto, 1991).

Epicauta (Epicauta) carmelita (Haag-Rutenberg, 1880) Lytta carmelita Haag-Rutenberg, 1880: 46 (*)

TERRA TYPICA: "Amer. centr., Neu-Granada, Mexico?" originalmente; "N. Grenada..." tras designación de lectotipo en la colección del British Museum of Natural History (Londres) (Pinto, 1982). Epicauta carmelita (Haag-Rutenberg, 1880): Dugès, 1886a: $582(*)$ Cantharis carmelita (Haag-Rutenberg, 1880): Champion, 1899: 174 (*)

COMENTARIOS TAXONÓMICOS: Existe el nombre no disponible E. carmelita Chevrolat in Dejean, 1837: 247 asociado a esta especie por Beauregard (1890) y Champion (1892). Especie incluida en el grupo de $E$. carmelita por Pinto (1991).

DisTRIBUCIÓN GEOGRÁFICA: Extendida desde el sur de México hasta Venezuela, con registros en Colombia, Costa Rica, Guatemala, Honduras, Nicaragua y Panamá (Pinto, 1991; Maes \& Huether, 2007). En México se ha citado en los estados de Chiapas, Oaxaca y Veracruz. La cita de "Palo Verde" (Champion, 1892; Pinto, 1991) podría referirse a la localidad de dicho nombre en Morelos (México) o en Costa Rica.

MÉxICo: Chiapas: Arriaga (Pinto, 1991); $13 \mathrm{~km}$ al NE de Chiapa de Corzo (Pinto, 1991); 10-12 mi. al N de Cintalapa
(Pinto, 1991); Ocozocuautla (Pinto, 1991); 16 km O Ocozocuautla (Pinto, 1991); $20 \mathrm{~km} \mathrm{O}$ Ocozocuautla (Pinto, 1991); 18 mi. O Ocozocuautla (Pinto, 1991); 26 km al SO de Ocozocuautla (Pinto, 1991); San Jerónimo, Volcán Tacana (Pinto, 1991); 16 km al S de Tonalá (Pinto, 1991). Oaxaca: Matías Romero (Pinto, 1991); Tehuantepec (Champion, 1892; Pinto, 1991); Ventosa (Pinto, 1991). Veracruz: Cotaxtla (Pinto, 1991); Lago Catemaco area (Pinto, 1991); Los Tuxtlas range (Pinto, 1991); Paso del Macho (Dugès, 1889e; Champion, 1892; Pinto, 1991); Playa Vicente (Champion, 1892; Pinto, 1991).

Epicauta (Epicauta) castadiva Pinto, 1991

Epicauta castadiva Pinto, 1991: 176 (*)

TERRA TYPICA: "14 mi. south of Valle Nacional, (4200’), Oaxaca, Mexico". Holotipo, en la colección de la California Academy of Sciences (San Francisco, EEUU) (fide Pinto, 1991).

COMENTARIOS TAXONÓMICOS: Especie incluida en el grupo de E. cinerea por Pinto (1991).

DisTRIBUCIÓN GEOGRÁFICA: Solamente conocida de la localidad típica en Oaxaca (Pinto, 1991).

MÉxICo: Oaxaca: $14 \mathrm{mi}$. al sur de Valle Nacional (Pinto, 1991).

Epicauta (Epicauta) cazieri Dillon, 1952

Epicauta cazieri Dillon, 1952: 394 (*)

TERRA TYPICA: "Sierra Blanca, El Paso Co., ...". Holotipo en la colección del American Museum of Natural History (EEUU), pero no tiene el no 28219 indicado por Werner (fide Pinto, 1991).

COMENTARIOS TAXONÓMICOS: Incluida en el grupo de E. bispinosa por Pinto (1975b; 1991).

DISTRIBUCIÓN GEOGRÁFICA: Especie distribuida por el norte de México y el sur de los EEUU (Pinto, 1991). En México se ha citado en Chihuahua, Nuevo León y San Luis Potosí.

MÉxICo: Chihuahua: 16 mi. al SE de Chihuahua (Pinto, 1975b). Nuevo León: $40 \mathrm{mi}$. al NO del cruce de entre la carretera 57 y la 60 (Pinto, 1975b); 79 mi. al SO de Linares (Pinto, 1975b). San Luis Potosí: Hidalgo (Pinto, 1975b).

Epicauta (Epicauta) cinctipennis (Chevrolat, 1834)

Lytta cinctipennis Chevrolat, 1834a: fasc. $3, \mathrm{n}^{\circ} 59$ (sin paginar) $\left(^{*}\right)$

TERRA TYPICA: "Zimapan" originalmente; "Mexico, Mineral del Zimapan..." tras designación de lectotipo (Pinto, 1982) en la colección del Museum Zoologicum Universitatis (Helsinki).

Cantharis cinctipennis (Chevrolat, 1834): Dugès, 1869a: $126\left(^{*}\right.$

Epicauta cinctipennis (Chevrolat, 1834): Dugès, 1886a: $582\left({ }^{*}\right)$

Epicauta ruidosana Fall in Fall et Cockerell, 1907: 258 (*)

TERRA TYPICA: “...North Fork of the Ruidoso, White Mountains, 7200 feet". Holotipo $\mathrm{n}^{\circ} 24295$ en la colección del Museum of Comparative Zoology (Harvard University, Cambridge, EEUU) (fide Werner, 1945; Pinto, 1991).

COMENTARIOS TAXONÓMICOS: Especie incluida en el grupo de E. cinctipennis por Pinto (1991). Pinto (1991) indica que los ejemplares de Arizona difieren en algunos caracteres poco constantes del resto de los ejemplares, aunque no propone ningún cambio taxonómico al respecto y mantiene la sinonimia de E. ruidosana con $E$. cinctipennis que propuso anteriormente (Pinto, 1982). El 
nombre Epicauta circunscripta Sturm, 1843: 175, asociado a E. cinctipennis por Champion (1892), o el de $E$. cicunscripta Klug in Dejean, 1837: 247, indicado en Beauregard (1890), son nombres no disponibles.

DisTrIBUCIÓN GEOGRÁfiCA: Extendida desde Morelos hasta Arizona (Pinto, 1991). En México se ha citado en los estados de Guanajuato, Hidalgo, Michoacán, Morelos, Nuevo León, Puebla, Querétaro y San Luis Potosí. Pinto (1991) no recoge las citas de Michoacán, Distrito Federal, Zacualtipán, San Miguelito y Durasnal de Champion (1892).

MÉxICo: Distrito Federal: Ciudad de México (Champion, 1892). Guanajuato: 3 mi. O Dolores Hidalgo (Pinto, 1991); Cerros de Guanajuato (Dugès, 1869b); Guanajuato (Dugès, 1869b, 1889e; Champion, 1892; Pinto, 1991); 22 mi. al NE de Guanajuato (Pinto, 1991); Quatemala [sic] (Pinto, 1991); 9 mi. al S de San Luis de la Paz (Pinto, 1991). Hidalgo: 6 mi. al NE de Jacala (Pinto, 1991); 14 mi. al S de Jacala (Pinto, 1991); Pachuca (Pinto, 1991); San Miguel (Pinto, 1991); Zacualtipán (Champion, 1892); Zimapán (Chevrolat, 1834a; Pinto, 1991); Minas de Zimapán (Dugès, 1869b; Champion, 1892). Michoacán: Quiroga (Dugès, 1889e; Champion, 1892). Morelos: $9 \mathrm{~km}$ al E de Cuernavaca (Pinto, 1991). Nuevo León: Aramberri (Pinto, 1991); Chipinque (Pinto, 1991); 4 mi. O El Cercado (Pinto, 1991); Cascada Cola de Caballo (Pinto, 1991); $23.6 \mathrm{mi}$. al SO de Linares (Pinto, 1991); $8 \mathrm{~km}$ al O de Santiago (Pinto, 1991). Puebla: Zacatlán (Pinto, 1991). Querétaro: 7 km al N de Querétaro (Pinto, 1991); 10 mi. al N de Querétaro (Pinto, 1991); San Miguelito (Champion, 1892). San Luis Potosí: Sierra de San Miguelito (Pinto, 1991). Sin localizar: Durasnal [posiblemente en Oaxaca según Selander \& Vaurie, 1962] (Champion, 1892).

\section{Epicauta (Epicauta) conferta (Say, 1824)}

Meloe confertus Say, 1824a: 281 (*)

TERRA TYPICA: “...vicinity of Council Bluffs and near the Rocky Mountains" "Missouri and Arkansa", originalmente; "Dallas, Texas", tras designación de neotipo (fide Pinto, 1991). Neotipo designado por Werner (1945) en la colección del Museum of Comparative Zoology (Harvard University, Cambridge, EEUU), aunque Pinto (1991) indica que este ejemplar no está identificado como tal en la serie que porta los datos correspondientes.

Henous techanus Haldeman, 1852: 377 (*)

TERRA TYPICA: No indicada en la descripción original, aunque Pinto (1991) basándose en otros datos de la obra sugiere: "Western Texas or en route between there and Santa Fe, New Mexico". El tipo está aparentemente perdido (fide Pinto, 1991).

Henous confertus (Say, 1824): LeConte, 1853: $330(*)$

Epicauta conferta (Say, 1824): Werner, 1945: 448 (*)

COMENTARIOS TAXONÓMICOS: Incluida en el grupo de E. conferta por Agafitei \& Selander (1980) y Pinto (1991). Beauregard (1890) consideró a H. techanus como una especie independiente. LeConte (1883: 167) escribe erróneamente Henous texanus y Dugès (1869a: 102) escribe Henons conferta.

DisTRIBUCIÓN GEOGRÁFICA: Extendida desde el extremo noreste de México hasta el centro de los EEUU (Pinto, 1991). En México se ha citado en los estados de Chihuahua, Coahuila y Nuevo León. Según Champion
(1891) y Borchmann (1917) las citas de H. confertus de Dugès $(1869 \mathrm{a})$ corresponden a $E$. cardui.

MÉxICo: Chihuahua: Ciudad Camargo (Pinto, 1991). Coahuila: San Pedro (Champion, 1891; Van Dyke, 1928; Vaurie, 1950). Nuevo León: Monterrey (Pinto, 1991).

\section{Epicauta (Epicauta) corvina (LeConte, 1858)}

Lytta corvina LeConte, 1858a: 21 (*)

TERRA TYPICA: "Arizona...: Llano Estacado..." originalmente; "Valley of the Gila", tras designación de lectotipo por Werner (1945). Lectotipo $\mathrm{n}^{\circ} 5097$, designado por Werner (1945), en la colección del Museum of Comparative Zoology (Harvard University, Cambridge, EEUU) (fide Pinto, 1991).

Epicauta corvina (LeConte, 1858): Horn, 1873: 102 (*)

COMENTARIOS TAXONÓMICOS: Incluida en el grupo de E. corvina por Pinto (1991). Epicauta nigerrima ha sido tratada como sinónimo de E. corvina por Beauregard (1890), Borchmann (1917) y Dillon (1952). Champion (1892) incluye el nombre no disponible Epicauta chevrolatii Dejean, 1837: 247 en la sinonimia de esta especie

DISTRIBUCIÓN GEOGRÁFICA: Extendida desde el norte de México hasta el norte de los EEUU (Pinto, 1991). En México se ha citado en los estados de Chihuahua, Coahuila, Durango, Sinaloa y Sonora. Según Pinto (1991) algunas de las citas de esta especie de Champion (1892) y Vaurie (1950) corresponden a E. nigerrima.

MÉxICo: Chihuahua: $12 \mathrm{mi}$. al N de Chihuahua (Pinto, 1991); El Sueco (Pinto, 1991); Villa Matamoros (Pinto, 1991); Santa Clara (Champion, 1892; Vaurie, 1950); Cañón de Santa Clara, 5 mi. al O de Parrita (Vaurie, 1950); Catarinas (Vaurie, 1950). Coahuila: Los Piños (Pinto, 1991). Durango: Canelas (Champion, 1892; Vaurie, 1950); Durango (Pinto, 1991); $7 \mathrm{mi}$. al O de Durango (Pinto, 1991); $20 \mathrm{mi}$. al O de Durango (Pinto, 1991); 25 mi. al S de Durango (Pinto, 1991); 2 mi. al S de La Zarca (Pinto, 1991); Nombre de Dios (Pinto, 1991); Villa (Ciudad) Lerdo (Champion, 1892; Vaurie, 1950; Pinto, 1991); Las Puentes (Vaurie, 1950); San Juan del Río (Vaurie, 1950); Palos Colorados (Vaurie, 1950); Providencia (Vaurie, 1950). Sinaloa: Escuinapa (Vaurie, 1950; Werner et al., 1966; Pinto, 1991). Sonora: Naco (Vaurie, 1950; Pinto, 1991).

\section{Epicauta (Epicauta) costata (LeConte, 1854)}

Lytta costata LeConte, 1854c: 84 (*)

TERRA TYPICA: "Frontera (Rio Grande)" en New Mexico según Pinto (1991). Holotipo $\mathrm{n}^{\circ}$ 4992, en la colección del Museum of Comparative Zoology (Harvard University, Cambridge, EEUU) (fide Pinto, 1991).

Pleuropompha costata (LeConte, 1854): LeConte, 1862: 273 (*)

Epicauta costata (LeConte, 1854): Beauregard, 1890: 504 (*)

COMENTARIOS TAXONÓMICOS: Especie todavía conservada en el género Pleuropompha por Werner et al. (1966), pero transferida al género Epicauta e incluida en el grupo de E. costata por Pinto (1991).

DisTRIBUCIÓN GEOGRÁFICA: Extendida desde el norte de México y el suroeste de los EEUU (Pinto, 1991). En México se ha citado en los estados de Chihuahua, Coahuila, Durango y Sonora. 
MÉXICo: Chihuahua: Camargo (Pinto, 1991); 15 mi. al S de Camargo (Pinto, 1991); $20 \mathrm{mi}$. al SO de Camargo (Vaurie, 1950; Pinto, 1991); 25 mi. al SO de Camargo (Vaurie, 1950); 42 mi. al SO de Camargo (Vaurie, 1950); Chihuahua (Pinto, 1991); Ciudad Ahumada (Selander, 1954b); Gallego (Pinto, 1991); 2 mi. al S de Nuevo Casas Grandes (Pinto, 1991); $15.5 \mathrm{~km}$ al S de Palomas (Pinto, 1991); 15 mi. al E de Parral (Vaurie, 1950); 18 mi. al N de Parral (Pinto, 1991); Primavera (Vaurie, 1950); Río San Pedro, 49 mi. al S de Chihuahua (Selander, 1954b); $5 \mathrm{~km}$ al S de Saucillo (Pinto, 1991); Sombreretillo (Pinto, 1991); Villa Aldama (Pinto, 1991); $2 \mathrm{~km}$ al S de Zaragoza (Pinto, 1991). Coahuila: 21 mi. al NO de Saltillo (Pinto, 1991); 8 mi. al N de Viesca (Dunas de Bilbao) (Pinto, 1991); Torreón (Pinto, 1991). Durango: 41 mi. al NE de Durango (Pinto, 1991); Villa Madero (Vaurie, 1950). Sonora: Campo Utah (Pinto, 1991); $18 \mathrm{mi}$. al SE de Guaymas (Pinto, 1991); Hermosillo (Pinto, 1991); 16 mi. al SO de Hermosillo (Pinto, 1991); 40 mi. al N de Hermosillo (Pinto, 1991).

\section{Epicauta (Epicauta) crassitarsis Maydell, 1935}

Epicauta crassitarsis Maydell, 1935: $72(*)$

TERRA TYPICA: “Tempe, Ariz.”. Holotipo nº 63924, en la colección del United States National Museum of Natural History (Washington D.C.) (fide Maydell, 1935; Pinto, 1991).

COMENTARIOS TAXONÓMICOS: Especie incluida en el grupo de E. cupraeola por Pinto (1991).

DISTRIBUCIÓN GEOGRÁFICA: Especie distribuida por el noroeste de México y extremo sur de Arizona (EEUU) (Pinto, 1991). Se ha citado en los estados de Sinaloa y Sonora.

MÉXICo: Sinaloa: 4 mi. al NO de Choix (Pinto, 1991); 39 mi. al NO de Culiacán (Pinto, 1991); 57 mi. al N de Culiacán (Pinto, 1991); $49 \mathrm{~km}$ al N de Elota (Pinto, 1991); 26 mi. al N de Pericos (Pinto, 1991). Sonora: Álamos (Pinto, 1991); 8 mi. al S de Álamos (Pinto, 1991); $10 \mathrm{mi}$. al O de Álamos (Pinto, 1991); 6 mi. al S de Benjamín Hill (Pinto, 1991); Ciudad Obregón (Werner et al., 1966; Pinto, 1991); 19.4 mi. al S de Estación Llano (Pinto, 1991); 26 mi. al SE de Guaymas (Pinto, 1991); Hermosillo (Pinto, 1991); 16 mi. al SO de Hermosillo (Pinto, 1991); 40 mi. al N de Hermosillo (Pinto, 1991); 12 km al S de Magdalena (Pinto, 1991); Navajoa (Werner et al., 1966; Pinto, 1991); 5 mi. al E de Navajoa (Pinto, 1991); 40 mi. al S de Santa Ana (Pinto, 1991).

\section{Epicauta (Epicauta) cupraeola (Dugès, 1869)}

\section{Cantharis cupraeola Dugès, 1869a: 112 (*)}

TERRA TYPICA: “... cerros de Guanajuato" originalmente; "Guanajuato, Mexico" tras la designación de neotipo. Pinto (1991) indica que el tipo original de Dugès está perdido, ya que el material indicado por Vázquez \& Zaragoza (1979) como tipo (n 799 de la colección del Insituto de Biología de la Universidad Nacional Autónoma de México) es de "Mirador, Ver.", localidad que no corresponde a la del material desigando por Dugès (1869a); sin embargo ZaragozaCaballero (1999) indica que en un manuscrito inédito Dugès indicó como única localidad para esta especie "Mirador" y que el ejemplar correspondiente se encuentra en la colección del Insituto de Biología de la Universidad Nacional Autónoma de México. En cualquier caso, Pinto (1991) designó neotipo en la colección del British Museum of Natural History (Londres) (fide Pinto, 1991).

Cantharis rufipedes Dugès, 1870: 163 (*)

TERRA TYPICA: "Michoacan, hacienda de la Noria..." originalemente; "10 miles south of Tzitzio (3200’), Michoacán, Mexico" tras la designación de neotipo (Pinto, 1991) en la colección de la California Academy of Sciences (San Francisco, EEUU).

Cantharis cinctella Dugès, 1877: 59 (*)

TERRA TYPICA: "Estado de Veracruz", originalmente; "7 km northeast of Catemaco, Veracruz, Mexico" tras la designación de neotipo (Pinto, 1991) en la colección de la California Academy of Sciences (San Francisco, EEUU).

Lytta subvittata Haag-Rutenberg, 1880: 47 (non Erichson, 1848) (*) TERRA TYPICA: "Mexico". Lectotipo con etiqueta de "Mexiko" designado por Pinto (1982) en la colección del Museum Zoologicum Universitatis (Helsinki).

Cantharis cupreola (Dugès, 1869): Horn, 1885: 107 (enmienda injustificada) (*)

Epicauta insignis Horn, 1885: $110\left(^{*}\right)$

TERRA TYPICA: “Arizona”. Lectotipo no 8097 designado por Werner (1945), inicialmente en la colección de la Academy of Natural Sciences of Philadelphia, posteriormente transferido al Museum of Comparative Zoology de Harvard (EEUU).

Epicauta cuprocela (Dugès, 1869): Dugès, 1886a: 582 (y error tipográfico) $(*)$

Epicauta cinctella (Dugès, 1877): Dugès, 1886a: $582(*)$

Epicauta rufipes (Dugès, 1870): Dugès, 1886a: 582 (y error tipográfico) (*)

Epicauta vittula Beauregard, 1889: ccxiii (nombre de substitución para Lytta subvittata Haag-Rutenberg, 1880) (*)

Epicauta cupreola (Dugès, 1869): Beauregard, 1890: 504 (*)

Cantharis insignis (Horn, 1885): Champion, 1899: $180\left({ }^{*}\right)$

COMENTARIOS TAXONÓMICOS: Especie incluida en el grupo de E. cupraeola por Pinto (1991). La variabilidad de esta especie ha generado mucha confusión taxonómica. Champion (1892), seguido por Denier (1935b), reduce $E$. cinctella a variedad de E. rufipedes, Pinto (1982) sinonimiza E. insignis con E. rufipedes, y finalmente Pinto (1991) sinonimiza E. rufipedes con E. cupraeola. Es muy posible que algunas de las diferentes denominaciones taxonómicas correspondan a variantes geográficas bien definidas que podrían considerarse subespecies. Dugès (1877) consigna en la sinonimia de C. cinctella el nombre no disponible Epicauta cinctella Dejean.

DISTRIBUCIÓN GEOGRÁFICA: Especie distribuida desde Costa Rica hasta el extremo meridional de los EEUU, incluyendo Nicaragua, Honduras, El Salvador, Guatemala y México (Pinto, 1991; Maes \& Huether, 2007). Su distribución en México es muy amplia y se ha registrado en casi todos los estados: Aguascalientes, Campeche, Chiapas, Chihuahua, Coahuila, Colima, Distrito Federal, Durango, Guanajuato, Guerrero, Jalisco, México, Michoacán, Morelos, Nayarit, Oaxaca, Puebla, Quintana Roo, San Luis Potosí, Sinaloa, Sonora, Veracruz, Yucatán y Zacatecas. Pinto (1991) señala que Vaurie (1950) relaciona citas de esta especie bajo el epígrafe de "especie desconocida".

MÉXICo: Aguascalientes: $10 \mathrm{mi}$. al S de Aguascalientes (Pinto, 1991); 5 mi. al N de Rincón de Ramos (Pinto, 1991). Campeche: Escarcega (Pinto, 1991). Chiapas: Ciudad Cuauhtémoc (Pinto, 1991); 31 mi. al S de Comitán (Pinto, 1991); El Ocotal (Pinto, 1991); $16 \mathrm{~km}$ al O de Ocozocuautla (Pinto, 1991); 19 mi. al O de Ocozocuautla (Pinto, 1991); 32 mi. al O de San Cristóbal (Pinto, 1991); Santa Isabel (Pinto, 1991); Simojovel (Pinto, 1991); Tapachula (Champion, 1892; 
Vaurie, 1950 sub E. rufipedes; Pinto, 1991); 72 mi. al E de La Ventosa (Pinto, 1991). Chihuahua: Catarinas (Vaurie, 1950 sub Epicauta sp.; Pinto, 1991); Chihuahua (Pinto, 1991). Coahuila: Saltillo (Pinto, 1991). Colima: Colima (Pinto, 1991); 10 mi. al O de Colima (Pinto, 1991); 12 mi. al SO de Colima (Pinto, 1991); $31 \mathrm{~km}$ al SE de Colomo (Pinto, 1991); 10 mi. al S de Tonila (Pinto, 1991); Tonila (Champion, 1892; Vaurie, 1950 sub E. rufipedes); Vulcano [Nevado de Colima] (Pinto, 1991). Distrito Federal: Teotihuacán (Pinto, 1991). Durango: 4 mi. al S de Casco (Pinto, 1991); Durango (Pinto, 1991); 5 mi. al O de Durango (Pinto, 1991); 8 mi. al NE de Durango (Pinto, 1991); 21 mi. al E de Durango (Pinto, 1991); Encino (Vaurie, 1950 sub Epicauta sp.; Pinto, 1991); Graceros (Pinto, 1991); 5 mi. al NO de Nombre de Dios (Pinto, 1991); 16 mi. al S de Nombre de Dios (Pinto, 1991); 5 mi. al S de Pueblo Nuevo (Pinto, 1991); Ventanas (Champion, 1892; Vaurie, 1950 sub E. rufipedes). Guanajuato: Guanajuato (Dugès, 1869a sub E. cupreola; Champion, 1892 sub E. cupreola; Vázquez \& Zaragoza, 1979 sub E. cupreola; Pinto, 1991). Guerrero: Acapulco (Champion, 1892; Vaurie, 1950 sub E. rufipedes; Pinto, 1991); Amula (Champion, 1892; Vaurie, 1950 sub E. rufipedes; Pinto, 1991); Chilpancingo (Champion, 1892; Vaurie, 1950 sub E. rufipedes; Pinto, 1991); 13 mi. al N de Chilpancingo (Pinto, 1991); $20 \mathrm{mi}$. al N de Chilpancingo (Pinto, 1991); 24 mi. al N de Chilpancingo (Pinto, 1991); 46 $\mathrm{km}$ al $\mathrm{N}$ de Chilpancingo (Pinto, 1991); Dos Arroyos (Champion, 1892; Vaurie, 1950 sub E. rufipedes); Iguala (Champion, 1892; Vaurie, 1950 sub E. rufipedes; Pinto, 1991); 30 mi. al S de Iguala (Pinto, 1991); $32 \mathrm{~km}$ al S de Iguala (Pinto, 1991); $43.7 \mathrm{~km}$ al NO de Ixtapa (Pinto, 1991); $53 \mathrm{~km}$ al O de La Unión (Pinto, 1991); 2.4 mi. al $\mathrm{N}$ de Mazatlán (Pinto, 1991); 5 mi. al NNE de Mexcala (Selander, 1954b, sub E. rufipedes; Pinto, 1991); Mochitlán (Champion, 1892; Vaurie, 1950 sub E. rufipedes; Pinto, 1991); Petaquillas (Pinto, 1991); Rincón (Champion, 1892; Vaurie, 1950 sub E. rufipedes; Pinto, 1991); 20 mi. al SE de Tecpan de Galeana (Pinto, 1991); Xalitla (Vaurie, 1950 sub E. rufipedes; Pinto, 1991); Zihuatanejo (Pinto, 1991). Jalisco: Ajijic (Pinto, 1991); $5 \mathrm{mi}$. al S de Atenquique (Pinto, 1991); Barra de Navidad (Pinto, 1991); $23.8 \mathrm{~km}$ al S de Chamela (Pinto, 1991); $17.6 \mathrm{~km}$ al N de Chamela (Pinto, 1991); Chapala (Pinto, 1991); $17 \mathrm{mi}$. al NO de Cihuatlán (Pinto, 1991); Guadalajara (Vaurie, 1950 sub E. rufipedes; Pinto, 1991); 4 mi. al SO de Guadalajara (Pinto, 1991); $10 \mathrm{~km}$ al E de Jocotepec (Pinto, 1991); Jamay (Pinto, 1991); $7.5 \mathrm{~km}$ al NE de Juchitlán (Pinto, 1991); $5 \mathrm{mi}$. al S de La Huerta (Pinto, 1991); $20 \mathrm{mi}$. al N de La Quemada (Pinto, 1991); Laguna de Zacoalco (Pinto, 1991); Sayula (Champion, 1892; Vaurie, 1950 sub E. rufipedes; Pinto, 1991); Tecolotlán (Pinto, 1991); Tuxpan (Pinto, 1991). México: Tejupilco (Pinto, 1991). Michoacán: Apatzingán (Pinto, 1991); $3 \mathrm{~km}$ al N de Capirio (Pinto, 1991); Huétamo (Champion, 1892; Vaurie, 1950 sub E. rufipedes; Pinto, 1991); Jiquilpan (Pinto, 1991); 1 mi. al N de Los Amates (Pinto, 1991); 6 mi. al SE de Morelia (Pinto, 1991); 4 mi. al E de Nueva Italia (Pinto, 1991); Oropeo (Pinto, 1991); 5 mi. al SO de Tiquicheo (Pinto, 1991); $10 \mathrm{mi}$. al S de Tzitzio (Pinto, 1991); Hacienda de la Noria, a 5 leguas de la Piedad (Dugès, 1870). Morelos: Acapulco (Pinto, 1991); $7 \mathrm{~km}$ al S de Alpuyeca (Pinto, 1991); Cuautla (Pinto, 1991); Cuernavaca (Champion, 1892; Vaurie, 1950 sub E. rufipedes; Pinto, 1991); $7 \mathrm{~km}$ al S de Cuernavaca (Pinto, 1991); $10 \mathrm{mi}$. al E de Cuernavaca (Pinto, 1991); 16 mi. al S de Cuernavaca (Pinto, 1991); Hermosa (Pinto, 1991); $6 \mathrm{mi}$. al SO de
Joncatepec (Pinto, 1991); Palo Bolero (Pinto, 1991); 3 mi. al O de Yautepec (Cañón de Lobos) (Pinto, 1991). Nayarit: Ahucatlán (Pinto, 1991); El Pichón (Pinto, 1991); Ixtlán del Río (Vaurie, 1950 sub E. rufipedes; Pinto, 1991); Jesús María (Pinto, 1991); San Juan Petotán (Pinto, 1991); 2 mi. al E de Santiago (Pinto, 1991); Tepic (Pinto, 1991). Oaxaca: 20 mi. al E de El Camarón (Pinto, 1991); Huajuapan de León (Pinto, 1991); 6 mi. al O de Jalapa del Marqués (Pinto, 1991); 11.6 mi. al O de Jalapa del Marqués (Pinto, 1991); 18 mi. al N de Juchitán [sic] (Pinto, 1991); La Ventosa (Pinto, 1991); 26 mi. al N de La Ventosa (Pinto, 1991); 14 mi. al N de Miahuatlán (Pinto, 1991); Mitla (Pinto, 1991); 10 mi. al N de Niltepec (Pinto, 1991); Oaxaca (Dugès, 1889e sub E. rufipedes; Champion, 1892; Vaurie, 1950 sub E. rufipedes; Pinto, 1991; Zaragoza-Caballero, 1999 sub E. rufipedes); $12 \mathrm{mi}$. al SE de Oaxaca (Pinto, 1991); 58 mi. al SE de Oaxaca (Pinto, 1991); Salina Cruz (Pinto, 1991); Tehuantepec (Champion, 1892; Vaurie, 1950 sub E. rufipedes; Pinto, 1991); Puente Tlacotepic (Pinto, 1991); $8 \mathrm{~km}$ al NO de Totolapan (Pinto, 1991); Tuxtepec (Pinto, 1991); Valerio Trujano (Pinto, 1991). Puebla: 1 mi. al O de Acatlán (Pinto, 1991); 11 mi. al SE de Acatlán (Pinto, 1991); 19 mi. al NO de Acatlán (Pinto, 1991); Atlixco (Champion, 1892; Vaurie, 1950 sub E. rufipedes; Pinto, 1991); Izúcar de Matamoros (Champion, 1892; Vaurie, 1950 sub E. rufipedes; Pinto, 1991); $10 \mathrm{mi}$. al S de Izúcar de Matamoros (Pinto, 1991); 12.5 mi. al N de Izúcar de Matamoros (Pinto, 1991); $15 \mathrm{~km}$ al O de Izúcar de Matamoros (Pinto, 1991); Puebla (Champion, 1892); Tehuacán (Pinto, 1991); $11 \mathrm{~km}$ al NO de Tehuitzingo (Pinto, 1991). Quintana Roo: 3-9 km al S de Tabi (Pinto, 1991). San Luis Potosí: 11 km al E de Ciudad Valles (Pinto, 1991). Sinaloa: Choix (Pinto, 1991); 4 mi. al NO de Choix (Pinto, 1991); 5.5 mi. al NO de Choix (Pinto, 1991); Concordia (Pinto, 1991); Coyotitlán (Werner et al., 1966 sub E. insignis); 6 mi. al S de Culiacán (Pinto, 1991); 21 mi. al S de Culiacán (Pinto, 1991); Elota (Pinto, 1991); $49 \mathrm{~km}$ al N de Elota (Pinto, 1991); 1 mi. al O de El Quelite (Pinto, 1991); Escuinapa (Pinto, 1991); Malpica (Pinto, 1991); Mazatlán (Pinto, 1991); 2 mi. al N de Mazatlán (Pinto, 1991); 5 mi. al N de Mazatlán (Pinto, 1991); $9 \mathrm{mi}$. al N de Mazatlán (Pinto, 1991); 15 mi. al SE de Mazatlán (Pinto, 1991); 61 mi. al N de Mazatlán (Pinto, 1991); Rosario (Pinto, 1991); $3.2 \mathrm{~km}$ al S de Rosario (Pinto, 1991); 5 mi. al SSE de Rosario (Pinto, 1991); Venadillo (Pinto, 1991); Villa Unión (Pinto, 1991). Sonora: 7 mi. al O de Álamos (Pinto, 1991); Ciudad Obregón (Werner et al., 1966 sub E. insignis; Pinto, 1991); Hermosillo (Pinto, 1991); 9 mi. al NNE de Imuris (Pinto, 1991); La Chiripa (Pinto, 1991); Minas Nuevas (Pinto, 1991); Navajoa (Pinto, 1991); 4.4 mi. al S de Oputo (Pinto, 1991); Saric (Pinto, 1991). Veracruz: Amate (Pinto, 1991); Atoyac (Pinto, 1991); $7 \mathrm{~km}$ al NE de Catemaco (Pinto, 1991); Córdoba (Pinto, 1991); 23 mi. al E de Córdoba (Pinto, 1991); Estación Experimental Cotaxtla (Pinto, 1991); Dos Amates (Pinto, 1991); El Bobo [ver Selander \& Vaurie, 1962] (Dugès, 1889e sub E. cinctella; Champion, 1892); $10 \mathrm{~km}$ al E de La Tinaja (Pinto, 1991); $7 \mathrm{mi}$. al SO de Minatitlán (Pinto, 1991); Mirador (16 km al NNE de Huatusco) [Hacienda El Mirador, Huatusco] (Dugès, 1889e; Pinto, 1991; Zaragoza-Caballero, 1999); San Andrés Tuxtla (Champion, 1892; Vaurie, 1950 sub E. rufipedes; Pinto, 1991); Vega de Alatorre (Pinto, 1991); Xalapa (Champion, 1892; Vaurie, 1950 sub E. rufipedes). Yucatán: Chichén Itzá (Pinto, 1991); Colonia Yucatán (Pinto, 1991). Dzibilchaltun (Pinto, 1991); Piste (Pinto, 1991); Temax (Champion, 1892; Vaurie, 
1950 sub E. rufipedes; Pinto, 1991); Tixkokob (Pinto, 1991); Yaxche (Pinto, 1991); Yaxcopoil (Pinto, 1991); $1 \mathrm{~km}$ al S de Xcalacoop (Pinto, 1991). Zacatecas: 8 mi. al S de Chalchihuites (Pinto, 1991).

Epicauta (Epicauta) curvicornis (Haag-Rutenberg, 1880)

Lytta curvicornis Haag-Rutenberg, 1880: $43(*)$

TERRA TYPICA: "Mexico. Mirador..." originalmente; "Mexico..." tras designación de lectotipo (Pinto, 1982) en la colección del British Museum of Natural History (Londres)

Lytta fauresi Haag-Rutenberg, 1880: 44 (*)

TERRA TYPICA: No indicada originalmente; "Mexico" de acuerdo con los sintipos del Museum Zoologicum Universitatis (Helsinki) (Pinto, 1991).

Macrobasis antennalis Dugès, 1881: 148, lám. 4, fig. 13 a-f (sine descriptio) (*)

TERRA TYPICA: no indicada, aunque Dugès (1889e) indica "Veracruz". La figura original podría utilizarse como tipo ya que Pinto (1991) indica que no ha encontrado material de esta especie de Dugès.

Epicauta antennalis (Dugès, 1881): Dugès, 1889e: 54 (*)

Macrobasis curvicornis (Haag-Rutenberg, 1880): Beauregard, 1890 $500(*)$

Epicauta curvicornis (Haag-Rutenberg, 1880): Champion, 1892: 406 (*) Cantharis curvicornis (Haag-Rutenberg, 1880): Champion, 1899: 175 (*)

COMENTARIOS TAXONÓMICOS: A lo largo de la taxonomía de esta esta especie se han venido arrastrando problemas causados por la existencia de dos nombres no disponibles: L. antennalis Deyrolle y L. fauresi Boucard. Haag-Rutenberg (1880: 44) hace disponible el nombre Lytta fauresi al proveerlo de una breve descripción. Dugès (1881) atribuye el nombre $M$. antenalis Deyrolle a una ilustración de E. curvicornis, haciendo, por lo tanto, que el nombre $M$. antenalis esté también disponible. Beauregard (1890) mantiene el nombre no disponible $C$. fouresi [sic] Boucard, entre los sinónimos de $E$. curvicornis, mientras que Borchmann (1917), Blackwelder (1945) y Pinto (1991) incluyen en la sinonimia de $E$. curvicornis el nombre $L$. fauresi, atribuyéndolo a Haag-Rutenberg (1880). Pinto (1991) indica que de los cuatro sintipos de L. fauresi que están en el Museum Zoologicum Universitatis (Helsinki) uno corresponde a E. obesa y los otros tres a la forma de coloración cinerea de E. curvicornis. Beauregard (1890) y Champion (1892) incluyen además en la sinonimia de E. curvicornis el nombre no disponible Epicauta nodicornis Klug in Dejean, 1837: 248.

Especie incluida en el grupo de E. curvicornis por Pinto (1991). Champion (1892) señala que existe variabilidad marcada en la pubescencia y punteado de la cabeza.

DisTRIBUCIÓN GEOGRÁFICA: Especie endémica de México (Pinto, 1991), ampliamente distribuida en los estados de Guerrero, Hidalgo, Michoacán, Morelos, Oaxaca, Puebla y Veracruz. Champion (1892, 1899), seguido por Borchmann (1917), indica que las citas de Lytta obesa de Herrera \& Mendoza (1866) y las de Dugès $(1870 ; 1889$ e) de Cantharis obesa corresponden a E. curvicornis.

MÉxICo: Guerrero: Amula (Champion, 1892; Pinto, 1991); Chilpancingo (Champion, 1892; Pinto, 1991); Iguala (Pinto,
1991); $38 \mathrm{~km}$ al O de Iguala (Pinto, 1991); Taxco (Pinto, 1991); $6 \mathrm{~km}$ al O de Taxco (Pinto, 1991); $10 \mathrm{~km}$ al E de Tixtla (Pinto, 1991). Hidalgo: Atotonilco el Grande (Herrera \& Mendoza, 1866 sub Lytta obessa; Dugès, 1869b sub E. obesa). Michoacán: Pátzcuaro (Pinto, 1991). Morelos: Cañón de Lobos (Pinto, 1991); Cuernavaca (Pinto, 1991); $6 \mathrm{mi}$. al E de Cuernavaca (Pinto, 1991); Tepoztlán (Pinto, 1991). Oaxaca: 9 mi. al SE de El Tule (Pinto, 1991); Guelatao (Pinto, 1991); Carretera 175, 6 mi. al N del cruce con la carretera 190 (Pinto, 1991); La Parada (Champion, 1892; Pinto, 1991); Miahuatlán (Pinto, 1991); Mitla (Pinto, 1991); Monte Albán (Pinto, 1991); 8 mi. al SE de Nochixtlán (Pinto, 1991); 9 mi. al SE de Nochixtlán (Pinto, 1991); Oaxaca (Champion, 1892; Pinto, 1991); 30 mi. al NO de Oaxaca (Pinto, 1991); San Pablo Huistepec (Pinto, 1991).

Puebla: Atlixco (Pinto, 1991); Izúcar de Matamoros (Champion, 1892; Pinto, 1991); Carretera 150, $10.2 \mathrm{mi}$. al O de del límite con Veracruz (Pinto, 1991); Los Molinos (Pinto, 1991); Puebla (Pinto, 1991). Veracruz: Xalapa (Champion, 1892; Pinto, 1991); Mirador [Hacienda El Mirador, Huatusco] (Haag-Rutenberg, 1880; Champion, 1892; Pinto, 1991); Orizaba (Champion, 1892; Pinto, 1991); Veracruz (Dugès, 1870 sub E. obesa, $1889 \mathrm{e}$ sub E. antennalis y E. obesa; Champion, 1892; Pinto, 1991). Sin localizar: Durasnal [posiblemente en Oaxaca según Selander \& Vaurie, 1962] (Champion, 1892; Pinto, 1991).

\section{Epicauta (Epicauta) delicata Mathieu, 1983}

Epicauta delicata Mathieu, 1983: 158 (*)

TERRA TYPICA: "Paila, Coahuila, México". Holotipo, en la colección de la California Academy of Sciences (San Francisco, EEUU) (fide Mathieu, 1983; Pinto, 1991).

COMENTARIOS TAXONÓMICOS: Incluida en el grupo de E. costata por Pinto (1991).

DistribuCión GEOGRÁFICA: Especie endémica de México (Pinto, 1991) que ha sido citada exclusivamente en Coahuila.

MÉXICo: Coahuila: Paila (Mathieu, 1983; Pinto, 1991); 8 mi. al N de Viesca (Dunas de Bilbao) (Pinto, 1991).

\section{Epicauta (Epicauta) diana Pinto, 1991}

Epicauta diana Pinto, 1991: 253 (*)

TERRA TYPICA: "6 mi. E Villa Union, Sinaloa, Mexico". Holotipo, en la colección de la California Academy of Sciences (San Francisco, EEUU) (fide Pinto, 1991)

COMENTARIOS TAXONÓMICOS: Especie incluida en el grupo de E. cupraeola por Pinto (1991).

Distribución GEOGRÁFICA: Especie endémica de México (Pinto, 1991). Citada en los estados de Oaxaca y Sinaloa.

MÉxICo: Oaxaca: Putla (Pinto, 1991). Sinaloa: Villa Unión (Pinto, 1991).

Epicauta (Epicauta) dugesi Werner, 1957

Epicauta dugesi Werner, 1957b: 108 (non E. dugesi Beauregard, 1889: nombre no disponible) (*)

TERRA TYPICA: "Tepic, Nayarit... Mazatlán, Sinaloa... Los Mochis, Sinaloa'

Epicauta tamara Adams \& Selander, 1979: 249 (*) syn. nov.

TERRA TYPICA: “Culiacán, Sinaloa, México...". Holotipo en la colección del American Museum of Natural History (EEUU) (fide Adams \& Selander, 1979; Pinto, 1991) 
COMENTARIOS TAXONÓMICOS: Incluida en el grupo de E. vittata por Adams \& Selander (1979), Agafitei \& Selander (1980) y Pinto (1991).

Werner (1957b) describe una especie nueva con el nombre de Epicauta dugesi, con la intención de validar una especie que él pensó erróneamente que había sido reconocida con anterioridad por Dugès (ver E. temexa). Adams \& Selander (1979) y Pinto (1991) señalaron que el nombre de Epicauta dugesi ya había sido utilizado por Beauregard (1889), quien propuso dicho nombre como substituto de E. vittata Dugès, 1886. El nombre de Dugès apareció en la lista de Meloidae que publicó en 1886a como "E. vittata Eug. Dug." sin más indicación. La intención de Dugès fue señalar la presencia de $E$. vittata (Fabricius, 1775) en México (hoy esas poblaciones se tratan como E. temexa) y no proponer un nombre nuevo como consideró Beauregard (1889), tal como el propio Dugès implica posteriormente al citar la especie como E. vittata (Fabricius, 1775) (Dugès 1889e: 87). En cualquier caso, el nombre de $E$. vittata Dugès, 1886, que no va acompañado de descripción ni de ilustraciones, es un nombre no disponible (incumple el artículo $12.1 \mathrm{del}$ Código Internacional de Nomenclatura Zoológica, CINZ, 2000). Por consiguiente, el nombre de substitución Epicauta dugesi Beauregard, 1889, también ha de ser considerado como un nombre no disponible (artículo 12.2.3 del Código Internacional de Nomenclatura Zoológica, CINZ, 2000). Adams \& Selander (1979), sin embargo, consideraron erróneamente que el nombre de Beauregard (1889) estaba disponible y que por lo tanto el de Werner (1957b) era un homónimo, por lo que describieron una especie nueva, E. tamara, para referirse a los ejemplares de Werner (1957b), que ahora incluimos como sinonimia subjetiva de $E$. dugesi.

Pinto (1991) indica que es muy probable que esta especie sea una variedad de E. unilineata.

DISTRIBUCIÓN GEOGRÁFICA: Especie con una distribución restringida al norte de México y suroeste de los EEUU (Pinto, 1991). Citado en los estados de Guerrero, Nayarit, Sinaloa y Sonora.

MÉXICo: Guerrero: $3.7 \mathrm{mi}$. al E de Marquelia (Pinto, 1991). Nayarit: Acaponeta (Adams \& Selander, 1979); 3 mi. al NO de Las Varas (Adams \& Selander, 1979); San Blas (Adams \& Selander, 1979); Tepic (Werner, 1957b sub E. dugesi; Adams \& Selander, 1979). Sinaloa: Concordia (Adams \& Selander, 1979); Culiacán (Adams \& Selander, 1979; Agafitei \& Selander, 1980; Pinto, 1991); 3 mi. al E de Culiacancito (Adams \& Selander, 1979); Los Mochis (Werner, 1957b sub E. dugesi; Adams \& Selander, 1979); Mazatlán (Werner, 1957b sub E. dugesi; Adams \& Selander, 1979); Río Presidio, cerca de Villa Unión (Adams \& Selander, 1979); Rosario (Adams \& Selander, 1979); Venedio [sic] (posiblemente Venadillo, ver Selander, 1960a) (Adams \& Selander, 1979). Sonora: Cocorit (Adams \& Selander, 1979); Presa de Mocuzari (Adams \& Selander, 1979); 18 mi. al E de El Puerto (Adams \& Selander, 1979).
Epicauta (Epicauta) emarginata Champion, 1892

Epicauta emarginata Champion, 1892: 426 (*)

TERra TYPICA: "MeXico, San Isidro in Coahuila" originalmente;

"San Isidro, Mexico..." tras designación de lectotipo (Pinto, 1982)

en la colección del British Museum of Natural History (Londres).

Cantharis emarginata (Champion, 1892): Champion, 1899: 177 (*)

Epicauta calcarata Werner, 1944: $70\left(^{*}\right)$

TERRA TYPICA: "Roswell, New Mexico". Holotipo no 26065 en la colección del Museum of Comparative Zoology (Harvard University, Cambridge, EEUU) (fide Werner, 1944; Pinto, 1991).

COMENTARIOS TAXONÓMICOS: Especie incluida en el grupo de E. sericans por Pinto (1991).

DistribuCión GEOGRÁfICA: Distribuida en una pequeña franja del norte de México y del sur de los EEUU (Pinto, 1991). En México se ha citado en los estados de Chihuahua y Coahuila.

MÉxICo: Chihuahua: 10 mi. al N de Jiménez (Pinto, 1991); 10 mi. al O de Jiménez (Pinto, 1991). Coahuila: San Isidro (Champion, 1892; Werner et al., 1966; Pinto, 1991).

\section{Epicauta (Epicauta) ferruginea (Say, 1824)}

Lytta ferruginea Say, 1824b: 298 (*)

TERRA TYPICA: “...on the Missouri”, "Missouri Territory" originalmente; "Cambridge, Nebraska", tras designación de neotipo. Neotipo designado por Werner (1945) en la colección del Museum of Comparative Zoology (Harvard University, Cambridge, EEUU). Epicauta ferruginea (Say, 1824): LeConte, 1866: $158\left(^{*}\right.$ )

COMENTARIOS TAXONÓMICOS: Especie incluida en el grupo de E. sericans por Pinto (1991). Pinto (1991) indica que en esta especie se pueden distinguir dos razas geográficas claramente separadas, que incluso podrían considerarse como especies distintas; sin embargo no efectúa ningún cambio taxonómico al respecto. Los ejemplares de Chihuahua corresponderían a la forma típica. Pinto (1991) considera que la sinonimia de L. sartorii con E. ferruginea que propone Champion (1892) y siguen Borchmann (1917) y Vaurie (1950), no es correcta, e incluye a L. sartorii en la sinonimia de E. sericans. Werner (1945) y Pinto (1991), al contrario que LeConte (1853) y Borchmann (1917), tampoco consideran correcta la inclusión de E. nigricornis Melsheimer, 1846, como sinónimo de E. ferruginea.

DistribuCión GeOGRÁfiCA: Desde el norte de México hasta el Canadá, con numerosos registros en los EEUU (Pinto, 1991). En México se ha citado en Aguascalientes, Chihuahua, Coahuila y Guerrero. Pinto (1991) no ha examinado todos los ejemplares correspondientes a las citas de esta especie de Vaurie (1950), por lo que alguna podría corresponder a $E$. sericans.

MÉXICo: Aguascalientes: Aguascalientes (Champion, 1892; Vaurie, 1950). Chihuahua: Madera (Vaurie, 1950; Pinto, 1991); 2 mi. al O de Matachic (Vaurie, 1950); Santa Clara (Vaurie, 1950). Coahuila: Monclova (Champion, 1892; Vaurie, 1950); Saltillo (Champion, 1892; Vaurie, 1950). Guerrero: Montañas de Álvarez (Champion, 1892; Vaurie, 1950). 
Epicauta (Epicauta) fortis Werner, 1944

Epicauta fortis Werner, 1944: 69 (*)

TERRA TYPICA: "Phoenix, Arizona". Holotipo no 26066, en la colección del Museum of Comparative Zoology (Harvard University, Cambridge, EEUU) (fide Werner, 1944; Pinto, 1991).

COMENTARIOS TAXONÓMICOS: Incluida en el grupo de E. sericans por Pinto (1991). Pinto (1991) indica que en esta especie se pueden distinguir dos razas geográficas muy diferenciadas, pero no efectúa ningún cambio taxonómico al respecto. Los ejemplares de Baja California, Baja California Sur, Chihuahua y Sonora corresponderían a la forma típica, mientras que los de Tamaulipas se incluirían en la otra raza.

DisTRIBUCIÓN GEOGRÁFICA: Especie distribuida desde en el norte de México y el sur de los EEUU (Pinto, 1991). En México se ha citado en Baja California, Baja California Sur, Chihuahua, Coahuila, Sonora y Tamaulipas.

MÉxICo: Baja California: $7.8 \mathrm{mi}$. al SE de Cataviña (Pinto, 1991); Rancho El Progreso (Pinto, 1991); 20 mi. al N de Rancho Mezquital (Werner et al., 1966; Pinto, 1991); 14 km al S de Rancho Rosarito (Pinto, 1991); 5 mi. al NO de San Agustín (Pinto, 1991). Baja California Sur: Rancho Tablón (Pinto, 1991); 8 mi. al N de Rancho Tablón (Pinto, 1991). Chihuahua: esquina NO de Chuhuichupa (Pinto, 1991); 13.7 $\mathrm{km}$ al O de La Norteña (Pinto, 1991). Coahuila: Guadalupe (Vaurie, 1950; Werner et al., 1966); La Gloria, al S de Monclova (Vaurie, 1950); Monclova (Werner et al., 1966). Sonora: $29.6 \mathrm{~km}$ al O de Naco (Pinto, 1991). Tamaulipas: al $\mathrm{S}$ de Media Luna (Pinto, 1991).

Epicauta (Epicauta) horni Champion, 1892

Cantharis cinerea Dugès, 1869c: 160 (non Meloe cinereus Forster, 1771 = Epicauta cinerea) $(*)$

TERRA TYPICA: "Guanajuato", en la descripción original; "Mexico.. Guanajuato..." tras designación de neotipo. Tipo original perdido, ya que según Pinto (1991) el ejemplar de la colección del Insituto de Biología de la Universidad Nacional Autónoma de México, n 2280 , indicado por Vázquez \& Zaragoza (1979) como tipo de E. cinerea Dugès, es un ejemplar de E. cardui de Michoacán. Pinto (1991) designa neotipo en la colección del British Museum of Natural History (Londres)

Cantharis vicina Dugès, 1881: 147 (non Lytta vicina Haag-Rutenberg, 1880 = Epicauta vicina) (nombre de substitución inadecuado) (*)

Epicauta vicina (Dugès, 1881): Dugès, 1886a: 582 (*)

Epicauta horni Champion, 1892: 412 (nombre de substitución para $C$. cinerea Dugès, 1869 y C. vicina Dugès, 1881) (*)

Cantharis horni (Champion, 1892): Champion, 1899: $180(*)$

COMENTARIOS TAXONÓMICOS: Incluida en el grupo de E. maculata por Pinto (1980, 1991). Horn (1885) consideró a $C$. vicina como posible sinónimo de E. sericans, pero Champion (1892), Borchmann (1917) y Pinto (1991) mantienen su validez con el nombre nuevo de Epicauta horni.

Distribución GEOGRÁFICA: Especie endémica de México (Pinto, 1991). Localizada en los estados de Colima, Guanajuato, Michoacán y Tamaulipas. Pinto (1991) no considera la cita de Michoacán de Champion (1892)
MÉxICo: Colima: Vulcano [Nevado de Colima] (Pinto, 1991). Guanajuato: Guanajuato (Dugès, 1869c, 1870; Champion, 1892; Pinto, 1991). Michoacán: (Dugès, 1889e; Champion, 1892; Zaragoza-Caballero, 1999). Tamaulipas: Tampico (Pinto, 1991).

Epicauta (Epicauta) hubbelli Werner, 1973

Epicauta (Epicauta) hubbelli Werner, 1973: 460 (*)

TERRA TYPICA: "Cumbre de Arriaga, Chiapas, Mexico...". Holotipo en la colección del Museum of Comparative Zoology (Harvard University, Cambridge, EEUU) (fide Werner, 1973; Pinto, 1991).

COMENTARIOS TAXONÓMICOS: Incluida en el grupo de E. curvicornis por Pinto (1991).

DistribuCión GEOGRÁfICA: Endemismo mexicano (Pinto, 1991), registrado en Chiapas y Oaxaca.

MÉxICo: Chiapas: 35 mi. al SO de Cintalapa (Pinto, 1991); Cumbre de Arriaga (Werner, 1973; Pinto, 1991); 2 mi. al E de Rizo de Oro (Pinto, 1991); 3 km al E de Rizo de Oro (Pinto, 1991); $16 \mathrm{~km}$ al SE de Tierra y Libertad (Pinto, 1991); Cerro Vernal, $16 \mathrm{~km}$ al S de Tonalá (Pinto, 1991); $75 \mathrm{mi}$. al SO de Tuxtla Gutiérrez (Pinto, 1991). Oaxaca: $33 \mathrm{~km}$ al N de Tepanetapec [sic] (Pinto, 1991).

Epicauta (Epicauta) insueta Werner, 1955

Epicauta insueta Werner, 1955: 11 (*)

TERRA TYPICA: "Toluca, Mexico, Mexico". Holotipo en la colección del American Museum of Natural History (EEUU) (fide Pinto, 1991).

COMENTARIOS TAXONÓMICOS: Especie incluida en el grupo de E. caviceps por Werner (1955) y Pinto (1991).

DisTRIBUCIÓN GEOGRÁFICA: De esta especie únicamente se conoce la serie típica, colectada en el Estado de México (Pinto, 1991).

MÉXICo: México: Toluca (Werner, 1955; Pinto, 1991).

\section{Epicauta (Epicauta) jimenezi Dugès, 1889}

Epicauta jimenezi Dugès, 1889e: 73 (*)

TERRA TYPICA: “Guadalajara”. Holotipo nº 2994 (IBUNAM 025), en la colección del Instituto de Biología de la Universidad Nacional Autónoma de México (fide Vázquez \& Zaragoza, 1979; Pinto, 1991).

Cantharis jimenezi (Dugès, 1889): Champion, 1899: $180(*)$

Epicauta nigropilosa Maydell, 1934: 332 (*)

TERRA TYPICA: “...Guadalajara, Mexico...”. Holotipo $n^{\circ}$ 8182, en la colección de la Academy of Natural Sciences of Philadelphia (EEUU) (fide Maydell, 1934; Pinto, 1991).

COMENTARIOS TAXONÓMICOS: Especie incluida en el grupo de E. corvina por Pinto (1991).

DISTRIBUCIÓN GEOGRÁFICA: Especie distribuida por el norte de México y extremo sur de los EEUU (Pinto, 1991). Se ha citado en los estados de Durango, Jalisco, Sinaloa y Sonora.

MÉXICO: Durango: Durango (Pinto, 1991); Ventanas (Champion, 1892; Vaurie, 1950); Canelas (Champion, 1892; Vaurie, 1950). Jalisco: Guadalajara (Dugès, 1889e; Champion, 1892; Maydell, 1934 sub E. nigropilosa; Vaurie, 1950; Vázquez \& Zaragoza, 1979; Pinto, 1991; Zaragoza-Caballero, 1999). Sinaloa: Mazatlán (Pinto, 1991); 3 km al E de Mazatlán 
(Pinto, 1991). Sonora: Alamos (Pinto, 1991); Cananea (Pinto, 1991); 19.4 mi. al S de Estación Llano (Pinto, 1991); Quirocoba (Pinto, 1991); Nogales (Pinto, 1991); San Bernardo (Pinto, 1991).

\section{Epicauta (Epicauta) laevicornis Werner, 1973}

Epicauta (Epicauta) laevicornis Werner, 1973: 458 (*)

TERRA TYPICA: "Mts. and cns. [mountains and canyons] N. Ajijic, Jalisco, Mexico, to 5400'...". Holotipo en la colección del Museum of Comparative Zoology (Harvard University, Cambridge, EEUU) (Werner, 1973; fide Pinto, 1991).

COMENTARIOS TAXONÓMICOS: Incluida en el grupo de E. curvicornis por Pinto (1991).

DisTRIBUCIÓN GEOGRÁFICA: Especie endémica de México (Pinto, 1991), distribuida por los estados de Colima, Guerrero, Jalisco, México y Michoacán.

MÉxICo: Colima: Vulcano [Nevado de Colima] (Pinto, 1991). Guerrero: Omilteme (Pinto, 1991). Jalisco: Ajijic (Pinto, 1991); al N de Ajijic (Werner, 1973; Pinto, 1991); Chapala (Pinto, 1991); 14 mi. al SO de Cocula (Pinto, 1991); La Floresta (Laguna de Chapala) (Pinto, 1991); Tecolotlán (Pinto, 1991). México: Temascaltepec (Pinto, 1991). Michoacán: Cotija (Pinto, 1991); Laguna de Chapala, al NO de Cojumatlán (Pinto, 1991); 6 mi. al O de Sahuayo (Pinto, 1991)

\section{Epicauta (Epicauta) leucocoma Champion, 1892}

\section{Epicauta leucocoma Champion, 1892: 425 (*)}

TERRA TYPICA: "MeXICO, Tepanistlahuaca..., Acapulco" originalmente; "Tepanistalabuaca, Mexico, ..." (sic) tras designación de lectotipo (Pinto, 1982) en la colección del British Museum of Natural History (Londres). La serie típica consta de dos ejemplares más. Cantharis leucocoma (Champion, 1892): Champion, 1899: 181 (*)

COMENTARIOS TAXONÓMICOS: Especie incluida en el grupo de E. cupraeola por Pinto (1991).

DISTRIBUCIÓN GEOGRÁFICA: Endemismo mexicano (Pinto, 1991), conocido en los estados de Guerrero, Jalisco, y Oaxaca.

MÉxICo: Guerrero: Acapulco (Champion, 1892; Pinto, 1991); 17 mi. al E de Acapulco (Pinto, 1991); $43.7 \mathrm{~km}$ al NO de Ixtapan (Pinto, 1991); 3.7 mi. al E de Marquelia (Pinto, 1991). Jalisco: Ocotlán, Laguna de Chapala (Pinto, 1991). Oaxaca: Juquila (Pinto, 1991); Tepanistlahuaca (Champion, 1892; Pinto, 1991).

\footnotetext{
Epicauta (Epicauta) maculata (Say, 1823)

Lytta maculata Say, 1824b: 298 (*)

TERRA TYPICA: "...Missouri and Arkansas... not uncommon near the Rocky Mountains"; "Indianola, Nebraska", tras designación de neotipo por Werner (1945) en la colección del Museum of Comparative Zoology (Harvard University, Cambridge, EEUU)

Lytta conspersa LeConte, 1853: 340 (*)

TERRA TYPICA: "Missouri Territory". Lectotipo $\mathrm{n}^{\circ} 5000$ designado por Werner (1945) en la colección del Museum of Comparative Zoology (Harvard University, Cambridge, EEUU) (fide Pinto, 1991)

Epicauta maculata (Say, 1823): LeConte, 1866: 158 (*)

Cantharis punctuata Dugès, 1870: $161\left(^{(*)}\right.$

TERRA TYPICA: “...cerros de Guanajuato...”. Tipo perdido (fide Pinto, 1980, 1991)
}

Cantharis maculata (Say, 1823): Gemminger et Harold, 1870: 2151 (*) Epicauta punctata (Dugès, 1870): Dugès, 1886a: 582 (y error tipográfico) $(*)$

Epicauta media Dugès, 1889e: $82(*)$

Terra TyPICA: "México". Tipo al parecer perdido (fide Pinto, 1991). Cantharis media (Dugès, 1889): Champion, 1899: 182 (*)

Epicauta nogales Werner, 1944: 65 (*)

TERRA TYPICA: "Nogales, Arizona". Holotipo no 26085 en la colección del Museum of Comparative Zoology (Harvard University, Cambridge, EEUU) (fide Werner, 1944; Pinto, 1991).

Comentarios taxonómicos: Incluida en el grupo de E. maculata por Pinto (1980, 1991). Horn (1885) establece la sinonimia entre $C$. punctuata y $L$. conspersa, mientras que Champion (1892) le sigue y trata E. conspersa como variedad de E. maculata. Ambos nombres se incluyen en la sinonimia de E. maculata por Borchmann (1917). Pinto (1980) establece la sinonimia de E. nogales. Epicauta pardalis, incluida con dudas en la sinonimia de E. maculata por Borchmann (1917), se considera una especie válida. Beauregard (1890) consideró a $E$. ocellata un sinónimo de $E$. maculata, pero trata a $E$. media como especie independiente (aunque le aplica el nombre no disponible de E. media Dugès, 1866). Burmeister (1881) menciona en la sinonimia de la especie de América meridional Epicauta adspersa (Klug, 1825) el nombre no disponible Epicauta conspersa Germar in Dejean. Dugès (1889e: 81) incluye un error tipográfico en el nombre del género: Epicauata punctuata. Zaragoza-Caballero (1999: 22) incluye el error tipográfico Epicauta punctulata.

Según Pinto (1980) los ejemplares de E. maculata de la Sierra Madre Occidental son diferentes y podrían constituir una especie independiente.

DisTRIBUCIÓN GEOGRÁFICA: Extendida desde el sur del Canadá hasta Guatemala, incluyendo gran parte de México y de los EEUU (Pinto, 1980, 1991). En México, se ha citado en los estados de Aguascalientes, Chihuahua, Coahuila, Distrito Federal, Durango, Guanajuato, Hidalgo, Jalisco, México, Michoacán, Morelos, Nuevo León, Oaxaca, Querétaro, San Luis Potosí, Sonora y Zacatecas. Algunas de las citas recogidas por Vaurie (1950) con el nombre de E. maculata podrían no corresponder a esta especie (San Luis Potosí). Vaurie (1950), además, indica citas bajo el nombre de E. normalis Werner, 1943, que no han sido asignadas a una especie concreta: Coahuila: La Rosa (Vaurie, 1950). Distrito Federal: 119 de Guadalupe (Vaurie, 1950). Jalisco: a 40 km de Guadalajara (Vaurie, 1950); de éstas, es muy posible que las dos últimas correspondan a E. abeona. Pinto (1991) indica que ha examinado dos ejemplares de Coahuila: "Posos de la Becerra, Quatro Cienegas Basin" (sic), que a pesar de su similitud con E. maculata, no pueden asignarse a ninguna especie conocida.

MÉxICo: Aguascalientes: $10 \mathrm{mi}$. al E de Aguascalientes (Pinto, 1980); Pabellón (Pinto, 1980). Chihuahua: 16 mi. al SE de Chihuahua (Vaurie, 1950 sub E. normalis); Delicias 
(Vaurie, 1950 sub E. normalis; Pinto, 1980); Madera (Vaurie, 1950 sub E. normalis; Pinto, 1980); Metachic (Vaurie, 1950 sub E. normalis; Pinto, 1980); cerca de Ojito (Pinto, 1980); 2 mi. al O de Pedernales (Pinto, 1980); Rosario (Pinto, 1980); Salaices (Pinto, 1980); 1 mi. al N de San Isidro (Pinto, 1980); San José Babícora (Vaurie, 1950 sub E. normalis; Pinto, 1980). Coahuila: (Hacienda de) Guadalupe (Pinto, 1980); Saltillo (Pinto, 1980); 11 mi. al NE de Saltillo (Pinto, 1980). Distrito Federal: Ciudad de México (Dugès, 1889e sub E. media; Champion, 1892; Vaurie, 1950). Durango: Donato Guerra (Pinto, 1980); Durango (Pinto, 1980); $21 \mathrm{mi}$. al NE de Durango (Pinto, 1980); 21 mi. al E de Durango (Pinto, 1980); Ciudad Lerdo (Champion, 1892; Vaurie, 1950; Pinto, 1980). Guanajuato: Celaya (Pinto, 1980); Guanajuato (Champion, 1892; Pinto, 1980; Zaragoza-Caballero, 1999 sub E. punctulata); Cerros de Guanajuato (Dugès, 1870); Irapuato (Champion, 1892; Vaurie, 1950; Pinto, 1980); San Miguel de Allende (Pinto, 1980); 1 mi. al N de Sauceda (Pinto, 1980); Silao (Zaragoza-Caballero, 1999 sub E. punctulata). Hidalgo: Tula (Champion, 1892; Vaurie, 1950; Pinto, 1980); 3 mi. al S de Zimapan (Pinto, 1980). Jalisco: Ciénega de Mata (Pinto, 1980); Guadalajara (Pinto, 1980); Lagos de Moreno (Pinto, 1980); 5 mi. al SE de Lagos de Moreno (Pinto, 1980); 8 km al E de Ojuelos (Pinto, 1980); San Cristóbal (Pinto, 1980). México: San Juan Teotihuacán (Pinto, 1980). Michoacán: Morelia (Dugès, 1889e; Champion, 1892; Vaurie, 1950); Quiroga (Dugès, 1889e; Champion, 1892; Vaurie, 1950). Morelos: Yautepec (Champion, 1892; Vaurie, 1950). Nuevo León: $20 \mathrm{~km}$ al S de Montemorelos (Pinto, 1980); $5 \mathrm{mi}$. al S de Sabinas Hidalgo (Pinto, 1980). Oaxaca: Oaxaca (Champion, 1892; Vaurie, 1950; Pinto, 1980). Querétaro: $15 \mathrm{~km}$ al N de Querétaro (Pinto, 1980); Querétaro (Champion, 1892; Vaurie, 1950). San Luis Potosí: Huasteca Potosina (Dugès, 1889e; Champion, 1892; Vaurie, 1950). Sonora: Magdalena (Werner et al., 1966; Pinto, 1980); Molinas (Pinto, 1980); Nogales (Pinto, 1980). Zacatecas: $23 \mathrm{mi}$. al SE de Guadalupe (Pinto, 1980); Sombrerete (Pinto, 1980); 16 mi. al SE de Zacatecas (Pinto, 1980).

Epicauta (Epicauta) major Pic, 1924

Epicauta cinctipennis var. major Pic, 1924: $2(*)$

TERRA TYPICA: "Guatemala" originalmente. Sintipos posiblemente en el Muséum National d'Histoire Naturelle (Paris) (fide Pinto, 1991).

COMENTARIOS TAXONÓMICOS: Especie incluida en el grupo de E. cinerea por Pinto (1991). Denier (1935b), seguido por Blackwelder (1945), consideró que E. c. var. major debería ser una raza de E. subatra. Pinto (1991: $191)$ eleva a especie a $E$. major e indica que se trata de una especie muy variable en coloración e incluso en algunos caracteres antenales, por lo que señala la necesidad de nuevos estudios en esta especie.

Dugès (1886a: 582) incluye el nombre E. marginata Eug. Dug. en una lista de Meloidae de México y posteriormente (Dugès, 1877: 59) describe la especie utilizando el nombre Cantharis marginata. Beauregard (1890) y Zaragoza-Caballero (1999) atribuyen a Dugès el nombre E. marginata, pero como el propio Dugès (1877: 59) incluye en la sinonimia el nombre E. marginata (Fab.) parece razonable pensar que Dugès se refiere en realidad a ejemplares identificados como E. marginata (Fabricius, 1775) y no a una especie nueva.

DisTRIBUCIÓN GEOGRÁFICA: Extendida desde el noreste de México hasta Panamá, con registros en Costa Rica, Guatemala, Honduras y Nicaragua (Pinto, 1991; Maes \& Huether, 2007). En México se ha citado en los estados de Campeche, Chiapas, Oaxaca, Quintana Roo, San Luis Potosí, Tabasco, Veracruz y Yucatán. Pinto (1991) señala que las citas de E. cinerea (Forster, 1771) y E. marginata (Fabricius, 1775) de Dugès (1877, 1889 e) y Champion (1892) y las de E. subatra de Denier (1935b), corresponden en realidad a E. major. Según Pinto (1991), el ejemplar identificado como tipo de $C$. basalis $(=E$. immaculata $)$ en la colección de la Universidad Nacional Autónoma de México (Vázquez \& Zaragoza, 1979) también es un ejemplar de E. major.

MÉxICo: Campeche: $4 \mathrm{~km}$ al E de Xpujil (Pinto, 1991). Chiapas: Ladera septentrional Cerro Bola (Pinto, 1991); 24 mi. al NO de Huixtla (Pinto, 1991); 2 mi. al S de Ixhuatan (Pinto, 1991); al N de de Jitotol (Pinto, 1991); La Cania Chansayas (Pinto, 1991); 18 mi. al O de Ocozocuautla (Pinto, 1991); 24 mi. al NO de Ocozocuautla (Pinto, 1991); 4 mi. al N de Pablo Nuevo Solistahuacan [sic] (Pinto, 1991); Palenque (Pinto, 1991); $10 \mathrm{~km}$ al S de Palenque (Pinto, 1991); cerca de Rincón (Pinto, 1991); San Cristóbal de las Casas (Pinto, 1991); Simojovel (Pinto, 1991); 7 mi. al SE de Simojovel (Pinto, 1991); Cañón del Sumidero (Pinto, 1991); Tenejapa (Pinto, 1991); 14 mi. al N de Tuxtla Gutiérrez (Pinto, 1991). Oaxaca: Juquila Mixes (Pinto, 1991); 25 mi. al N de Matías Romero (Pinto, 1991); Oaxaca (Champion, 1892 sub E. cinerea); Temescal (Pinto, 1991). Puebla: Puebla (Champion, 1892 sub E. cinerea). Quintana Roo: 2 mi. al S de Felipe Carrillo (Pinto, 1991). San Luis Potosí: $52 \mathrm{~km}$ al E de Ciudad del Maíz (Pinto, 1991); Cascada El Salto (Pinto, 1991); 15 mi. al SO de Tamazunchale (Pinto, 1991); Tierra Blanca (Pinto, 1991). Tabasco: Cárdenas (Pinto, 1991); Teapa (Champion, 1892 sub E. cinerea). Veracruz: Atoyac (Champion, $1892 \mathrm{sub}$ E. cinerea; Pinto, 1991); $15 \mathrm{mi}$. al S de Catemaco (Pinto, 1991); al SE de del Citlaltepetl (Pinto, 1991); Córdoba (Champion, 1892 sub E. cinerea; Pinto, 1991); 10.4 mi. al SE de Córdoba (Pinto, 1991); 5 mi. al E de Córdoba (Pinto, 1991); Fortín de las Flores (Pinto, 1991); 1 mi. al O de Fortín de las Flores (Pinto, 1991); 2 mi. al N de Fortín de las Flores (Pinto, 1991); Xalapa (Champion, 1892 sub E. cinerea; Pinto, 1991); Lago Catemaco (Pinto, 1991); Estación Biológica de Los Tuxtlas (Pinto, 1991); Mirador [Hacienda El Mirador, Huatusco] (Pinto, 1991); Misantla (Champion, 1892 sub E. cinerea); Nanchital (Pinto, 1991); Orizaba (Pinto, 1991); 5 mi. al O de Orizaba (Pinto, 1991); Playa Vicente (Champion, 1892 sub E. cinerea); Presidio (Pinto, 1991); Río Metlac cerca de Fortín de las Flores (Pinto, 1991); San Andrés Tuxtla (Champion, 1892 sub E. cinerea); 2 mi. al N de Santiago Tuxtla (Pinto, 1991); $4 \mathrm{mi}$. al N de Sontecomapan (Pinto, 1991); 7 mi. al N de Sontecomapan (Pinto, 1991); Temescal (Pinto, 1991); Veracruz (Zaragoza-Caballero, 1999 sub E. marginata). Yucatán: X-Can (Pinto, 1991); Nuevo X-Can (Pinto, 1991). Sin localizar: Chinautla (Champion, 1892 sub E. cinerea). 
Epicauta (Epicauta) mexicana (Dugès, 1889)

Henous mexicanus Dugès, 1889d: 37 (*)

TERRA TYPICA: "Tulancingo" originalmente, pero en la etiqueta del lectotipo la localidad es: "Mexico". Lectotipo designado por Pinto (1991), en la colección del Magyar Természettudományi Múzeum de Budapest (Hungría).

Poreospasta mexicana (Dugès, 1889): Blackwelder, 1945: 487 (*)

Epicauta mexicana (Dugès, 1889): Pinto, 1991: 196 (*)

COMENTARIOS TAXONÓMICOS: Incluida en el grupo de E. cinerea por Pinto (1991).

DisTRIBUCIÓN GEOGRÁFICA: Endemismo mexicano (Pinto, 1991). Se ha citado en los estados de Hidalgo y Veracruz.

MÉXICo: Hidalgo: Tulancingo (Dugès, 1889d; Champion, 1892; Pinto, 1991; Zaragoza-Caballero, 1999). Veracruz: Misantla (Champion, 1892; Pinto, 1991).

\section{Epicauta (Epicauta) mixta Dugès, 1889}

Epicauta mixta Dugès, 1889: 83 (*)

TERRA TYPICA: "Oajaca". Holotipo no 2208 (IBUNAM 027), en la colección del Instituto de Biología de la Universidad Nacional Autónoma de México (fide Vázquez \& Zaragoza, 1979; Pinto, 1991).

COMENTARIOS TAXONÓMICOS: Especie incluida en el grupo de E. corvina por Pinto (1991). Posee una gran variabilidad en la coloración de la pubescencia y en la presencia de tubérculos glabros en el pronoto (Pinto, 1991). Champion (1892), seguido por Borchmann (1917), consideró erróneamente a E. mixta como sinónimo de E. neglecta (Pinto, 1982). Dugès (1886a: 582) publicó con anterioridad el nombre de E. mixta aunque sin adjuntar una descripción, creando un nombre no disponible. Champion (1892) indica el nombre no disponible Epicauta anilis Sturm, 1843: 175 en la sinonimia de E. neglecta, que correspondería en realidad a E. mixta.

Distribución GEOGRÁFICA: Especie endémica de México (Pinto, 1991). Citada en los estados de Chiapas, Distrito Federal, Guanajuato, Guerrero, Hidalgo, Jalisco, México, Michoacán, Morelos, Oaxaca y Veracruz. Pinto (1982, 1991) señala que parte de las citas de HaagRutenberg (1880) y casi todas las de Champion (1892) de E. neglecta corresponden a E. mixta.

MÉxICO: Chiapas: Berriozábal (Pinto, 1991); 11 mi. al E de Chiapa de Corzo (Pinto, 1991); 13 mi. al NE de Chiapa de Corzo (Pinto, 1991); El Sumidero (Pinto, 1991); $20 \mathrm{~km}$ al S de Ocozocuautla (Pinto, 1991); $20 \mathrm{~km}$ al O de Ocozocuautla (Pinto, 1991); Río de La Venta (Pinto, 1991); San Cristóbal (Pinto, 1991); 3 mi. al N de San Cristóbal (Pinto, 1991); 17 km al O de Tuxtla Gutiérrez (Pinto, 1991). Distrito Federal: Ciudad de México (Champion, 1892; Pinto, 1991); Pedregal (Pinto, 1991); San Jerónimo (Pinto, 1991); Tizapan (Pinto, 1991). Guanajuato: Moroleón (Dugès, 1889e; Pinto, 1982). Guerrero: Chilpancingo (Champion, 1892; Pinto, 1991); Omilteme (Champion, 1892; Pinto, 1991); Taxco (Pinto, 1991); $6 \mathrm{~km}$ al O de Taxco (Pinto, 1991); $13 \mathrm{~km}$ al $\mathrm{N}$ de Taxco (Pinto, 1991); Xucumanatlán (Champion, 1892; Pinto, 1991). Hidalgo: Guadalupe (Pinto, 1991). Jalisco: $4 \mathrm{mi}$. al O de
Mazamitla (Pinto, 1991). México: Coatlinchan (Pinto, 1991); Temascaltepec (Pinto, 1991); $11 \mathrm{mi}$. al O de Texcoco (Pinto, 1991). Michoacán: Carapan (Pinto, 1991); $10 \mathrm{mi}$. al O de Jiquilpan (Pinto, 1991); Pátzcuaro (Pinto, 1991); 3 km al E de Quiroga (Pinto, 1991). Morelos: Cuernavaca (Pinto, 1991); 9 km al E de Cuernavaca (Pinto, 1991). Oaxaca: La Parada (Champion, 1892; Pinto, 1991); Monte Albán (Pinto, 1991); 8 mi. al E de Nochixtlán (Pinto, 1991); Oaxaca (Dugès, 1889e; Champion, 1892; Vázquez \& Zaragoza, 1979; Pinto, 1982, 1991; Zaragoza-Caballero, 1999); $10 \mathrm{~km}$ al NE de Oaxaca (Pinto, 1991). Veracruz: Xalapa (Champion, 1892; Pinto, 1991). Sin localizar: La Herradura [Distrito Federal o San Luis Potosí] (Pinto, 1991); Soltepec [Puebla o Tlaxcala, ver Selander \& Vaurie, 1962] (Champion, 1892; Pinto, 1991).

Epicauta (Epicauta) modesta (Haag-Rutenberg, 1880) Lytta modesta Haag-Rutenberg, 1880: $53\left(^{*}\right)$

TERRA TYPICA: "Mexico". Holotipo en la colección del Zoologische Staatssammlung (Munich) (fide Pinto, 1991).

Epicauta modesta (Haag-Rutenberg, 1880): Beauregard, 1890: 508 (*)

Cantharis modesta (Haag-Rutenberg, 1880): Champion, 1899: 183 (*)

Comentarios taXonómicos: Incluida en el grupo de E. carmelita por Pinto (1991).

DisTRIBUCIÓN GEOGRÁFICA: De esta especie únicamente se conoce el holotipo, capturado en México pero sin más datos (Pinto, 1991).

MÉXICO: (Haag-Rutenberg, 1880; Pinto, 1991).

\section{Epicauta (Epicauta) nigerrima (Dugès, 1870)}

Cantharis nigerrima Dugès, 1870: $162\left({ }^{*}\right)$

TERRA TYPICA: “...en una calle de León (Estado de Guanajuato)...; Mineral del Cedro". Serie típica no identificada, posiblemente una parte se encuentre en el British Museum of Natural History (Londres) (fide Pinto, 1991).

Epicauta nigerrima (Dugès, 1870): Dugès, 1886a: 582 (*)

Cantharis nigerrima (Dugès, 1870): Champion, 1899: $183(*)$

COMENTARIOS TAXONÓMICOS: Especie incluida en el grupo de E. corvina por Pinto (1991). Fue incluida en la sinonimia de E. corvina por Horn (1885), Champion (1892, 1899) y Borchmann (1917), hasta que Pinto (1991) vuelve a reconocer su estatus específico.

Distribución GEOGRÁfiCA: Especie endémica de México (Pinto, 1991). Citada en los estados de Aguascalientes, Durango, Guerrero, Guanajuato, Jalisco, Michoacán, Nuevo León, Puebla, Querétaro, Sinaloa, Veracruz y Zacatecas. Pinto (1991) señala que esta especie ha sido citada por Horn (1885), Champion (1892) y Werner (1945) con el nombre de E. corvina (Pinto, 1991).

MÉXICo: Aguascalientes: $10 \mathrm{mi}$. al S de Aguascalientes (Pinto, 1991). Durango: Durango (Pinto, 1991); $6 \mathrm{mi}$. al S de Durango (Pinto, 1991); 21 mi. al NE de Durango (Pinto, 1991); 30 mi. al NO de Otinapa (Pinto, 1991); 38.3 mi. al SO de Parral (Pinto, 1991); Reserva La Hichilia (Pinto, 1991). Guanajuato: Guanajuato (Dugès, 1889e; Champion, 1892 sub E. corvina; Zaragoza-Caballero, 1999); León (Dugès, 1870; Champion, 1892 sub E. corvina; Vaurie, 1950 sub E. corvina; Pinto, 1991); Mineral del Cedro (Dugès, 1870; Champion, $1892 \mathrm{sub}$ 
E. corvina; Vaurie, 1950 sub E. corvina); Tupátaro (ZaragozaCaballero, 1999). Jalisco: Chapala (Pinto, 1991); Tizapan (Pinto, 1991); Villa Corona (Lago de Zacoalco) (Pinto, 1991). Michoacán: Cotija (Pinto, 1991); La Piedad (Pinto, 1991); 9 $\mathrm{km}$ al NE de Pátzcuaro (Pinto, 1991); Tuxpan (Pinto, 1991). Nuevo León: $10 \mathrm{mi}$. al N de Providencia (Pinto, 1991) Puebla: Puebla (Champion, 1892 sub E. corvina; Vaurie, 1950 sub E. corvina; Pinto, 1991). Querétaro: Querétaro (Pinto, 1991). Sinaloa: Esquinapa (Pinto, 1991). Veracruz: Sin precisar (Dugès, 1889e; Champion, 1892 sub E. corvina; Vaurie, 1950 sub E. corvina). Zacatecas: Fresnillo (Vaurie, 1950 sub E. corvina).

\section{Epicauta (Epicauta) nigritarsis (LeConte, 1853)}

Lytta nigritarsis LeConte, 1853: 340 (*)

TERRA TYPICA: "Mexican Boundary" originalmente; "Mexican Boundary, Texas" tras designación de lectotipo. Lectotipo $\mathrm{n}^{\circ} 4998$, designado por Werner (1945) en la colección del Museum of Comparative Zoology (Harvard University, Cambridge, EEUU) (fide Pinto, 1991)

Epicauta nigritarsis (LeConte, 1853): Horn, 1873: 100 (*)

Epicauta hesitata Dillon, 1952: 398 (*)

TERRA TYPICA: "Belton, Texas...”. Holotipo no 2041 en la colección de la Texas Agricultural and Mechanical University (EEUU) (fide Pinto, 1991).

COMENTARIOS TAXONÓMICOS: Especie incluida en el grupo de E. nigritarsis por Pinto (1991). Pinto (1982) establece la sinonimia de E. hesitata con E. nigritarsis.

DisTRIBUCIÓN GEOGRÁFICA: Se distribuye por el extremo noreste de México y el sur de los EEUU (Pinto, 1991). Citada en los estados de Coahuila, Nuevo León y Tamaulipas.

MÉXICo: Coahuila: Nueva Rosita (Pinto, 1991). Nuevo León: Apodaca (Pinto, 1991); $10 \mathrm{mi}$. al NE de Apodaca (Pinto, 1991); Cañón Huasteca (cerca de Monterrey) (Pinto, 1991); Linares (Pinto, 1991); Puerto de Mamulique (Pinto, 1991); Monterrey (Pinto, 1991); Nicolás de Los Garza (Pinto, 1991); Sabinas Hidalgo (Pinto, 1991); $17 \mathrm{~km}$ al N de Sabinas Hidalgo (Pinto, 1991). Tamaulipas: Ciudad Victoria (Pinto, 1991); 47 $\mathrm{km}$ al S de Ciudad Victoria (Pinto, 1991); $18 \mathrm{mi}$. al N de Ciudad Victoria (Pinto, 1991); Guemez (Pinto, 1991); $29 \mathrm{~km}$ al S de Hidalgo (Pinto, 1991); Matamoros (Pinto, 1991); Nuevo Laredo (Pinto, 1991); San Fernando (Pinto, 1991).

Epicauta (Epicauta) obesa (Chevrolat, 1834)

Lytta obesa Chevrolat, 1834b: fasc. $4, \mathrm{n}^{\circ} 81$ (sin paginar) (*)

TERRA TYPICA: “Toulepeck". Dos posibles sintipos en la colección del Museum Zoologicum Universitatis (Helsinki) (fide Pinto, 1991).

Cantharis obesa (Chevrolat, 1834): Dugès, 1869b: 128 (confusión con E. curvicornis) $(*)$

Lytta mus Haag-Rutenberg, 1880: 55 (*)

TERRA TYPICA: "Mexico, Miranda". Un sintipo muy deteriorado en el British Museum of Natural History (Londres) (fide Pinto, 1991).

Epicauta obesa (Chevrolat, 1834): Dugès, 1886a: 582 (*)

Cantharis mus (Haag-Rutenberg, 1880): Beauregard, 1890: $495(*)$

Epicauta auricomans Champion, 1892: 424 (*)

Terra TyPiCA: "MeXico, Saltillo in Coahuila..., Almolonga, Oaxaca..." originalmente; "Oaxaca, Mexico..." tras designación de ejemplar ilustrado por Champion (1892: lám. 19, fig. 24) como lectotipo (Pinto, 1982) en la colección del British Museum of Natural History (Londres).

Cantharis auricomans (Champion, 1892): Champion, 1899: 172 (*)
Epicauta ficta Werner, 1949b: $100(*)$

TERRA TYPICA: "Broken Bow, McCurtain Co., Oklahoma". Holotipo $\mathrm{n}^{\circ} 28223$ en la colección del Museum of Comparative Zoology (Harvard University, Cambridge, EEUU) (fide Werner, 1949b, Pinto, 1991).

Epicauta pseudosolani Dillon, 1952: 395 (*)

TERRA TYPICA: "College Station, Texas...”. Holotipo en la colección de la Texas Agricultural and Mechanical University (EEUU) (fide Pinto, 1991).

COMENTARIOS TAXONÓMICOS: Especie incluida en el grupo de E. cinerea por Pinto (1991). Beauregard (1890) incluye en la sinonimia de C. mus el nombre no disponible E. brevicornis Chevrolat in coll. y en la de E. obesa incluye el nombre E. grisea Dejean, 1837: 247, que tampoco es un nombre disponible. Champion (1892) vuelve a incluir en la sinonimia de E. obesa los dos nombres no disponibles anteriores. Borchmann (1917) trata a $E$. auricomans como especie independiente, pero Pinto (1982) establece la sinonimia de E. auricomans, E. ficta y E. pseudosolani con E. obesa. Pinto (1991) indica que existe una gran variabilidad intraespecífica en la pilosidad del cuerpo (longitud y coloración), en la de las patas, en los espolones tibiales posteriores (anchura) y en la estructura de las antenas. En algunos casos la variabilidad es independiente de la procedencia geográfica, pero en otros (pilosidad de las patas, estructura de las antenas) podría corresponder a variantes geográficas definidas. Herrera \& Mendoza (1866: 266) escriben erróneamente el nombre Lytta obessa.

DisTRIBUCIÓN GEOGRÁfICA: Extendida desde Veracruz y Oaxaca hasta el sureste del Canadá, con numerosas citas en el este de los EEUU (Pinto, 1991). En México se ha citado en Coahuila, Distrito Federal, Hidalgo, Jalisco, México, Michoacán, Morelos, Nuevo León, Oaxaca, Puebla, Querétaro, San Luis Potosí, Tamaulipas y Veracruz. Borchmann (1917) y Blackwelder (1945) señalan que las citas de E. obesa de Herrera \& Mendoza (1866) y parte de las de Dugès (1869a, 1889e) corresponden a $E$. curvicornis.

MÉxICo: Coahuila: Saltillo (Champion, 1892 sub E. auricomans; Vaurie, 1950). Distrito Federal: (Pinto, 1991). Hidalgo: Atotonilco el Grande (Dugès, 1869b); Chapulhuacán (Pinto, 1991). Jalisco: 3 mi. al O de Atotonilco (Pinto, 1991); $18 \mathrm{mi}$. al S de Guadalajara (Pinto, 1991). México: Toulepeck [Tultepec ](Chevrolat, 1834b; Champion, 1892). Michoacán: 15 mi. al N de Jiquilpan (Pinto, 1991); Lago de Cuitzeo (Pinto, 1991). Morelos: Acapulco [no localizado] (Pinto, 1991). Nuevo León: Apodaca (Pinto, 1991); Aramberri-Zaragoza (Pinto, 1991); Bustamonte [sic] (Pinto, 1991); Chipinque (Pinto, 1991); Cascada Cola de Caballo (Pinto, 1991); $10 \mathrm{mi}$. al O de Linares (Pinto, 1991); 20 mi. al O de Linares (Pinto, 1991); $18 \mathrm{mi}$. al SO de Linares (Pinto, 1991); $23 \mathrm{mi}$. al SO de Linares (Pinto, 1991); Monterrey (Pinto, 1991); 20 mi. al S de Monterrey (Pinto, 1991); Villa de García (Pinto, 1991); 3 km al SE de Villa de García (Pinto, 1991); Villa Santiago (Pinto, 1991); Zaragoza (Pinto, 1991). Oaxaca: Almolonga (Champion, 1892 sub E. auricomans; Vaurie, 1950); Oaxaca 
(Champion, 1892, y también sub E. auricomans; Vaurie, 1950); Temescal (Pinto, 1991). Puebla: Puebla (Pinto, 1991). Querétaro: Miranda (Haag-Rutenberg, 1880 sub Lytta mus); 7 km al N de Querétaro (Pinto, 1991); 3 mi. al O de Querétaro (Pinto, 1991). San Luis Potosí: Cascada El Salto (Pinto, 1991); $30 \mathrm{~km}$ al S de Huizache (Pinto, 1991); Tamazunchale (Pinto, 1991); 20 mi. al N de Tamazunchale (Pinto, 1991); Xilitla (Pinto, 1991). Tamaulipas: Río Carrizal (Pinto, 1991); $20 \mathrm{~km}$ al SE de Soto La Marina (Pinto, 1991); $40 \mathrm{~km}$ al N de Soto La Marina (Pinto, 1991). Veracruz: Chocama [sic] (Pinto, 1991); Chicontepec (Pinto, 1991); Coatepec (Pinto, 1991); Córdoba (Champion, 1892; Pinto, 1991); Cosmatepec [sic] (Pinto, 1991); 4 mi. al NE de Cosmatepec [sic] (Pinto, 1991); Fortín de las Flores (Pinto, 1991); Hacienda El Mirador (Champion, 1892); Huatusco (Pinto, 1991); $2 \mathrm{mi}$. al S de Huatusco (Pinto, 1991); 5 mi. al NE de Independencia (Pinto, 1991); Xalapa (Champion, 1892; Pinto, 1991); Orizaba (Champion, 1892; Pinto, 1991); Xico (Pinto, 1991).

\section{Epicauta (Epicauta) occipitalis Werner, 1949}

Epicauta occipitalis Werner, 1949b: $106\left({ }^{*}\right)$

TERRA TYPICA: "20 mi. N. of Mesquital, Lower California". Holotipo $\mathrm{n}^{\mathrm{o}} 6126$ en la colección de la California Academy of Sciences (San Francisco, EEUU) (fide Werner, 1949b; Pinto, 1991).

COMENTARIOS TAXONÓMICOS: Especie incluida en el grupo de E. caviceps por Werner (1955) y Pinto (1991). Pinto (1991) indica que en Rosarito se encuentra una especie diferente, aún sin describir, que aunque superficialmente similar a E. occipitalis, podría estar relacionada con E. afoveata.

DisTRIBUCIÓN GEOGRÁFICA: Especie endémica de la Península de Baja California (Pinto, 1991).

MÉxICo: Baja California: $20 \mathrm{mi}$. al $\mathrm{N}$ de Mesquital (Werner, 1949b; Pinto, 1991); Rosarito (Pinto, 1991); $17 \mathrm{~km}$ al S de Rosarito (Pinto, 1991). Baja California Sur: 20 mi. al S de El Arco (Pinto, 1991); El Arco (Werner, 1949b); $6.1 \mathrm{~km}$ al S de Guerrero Negro (Pinto, 1991).

\section{Epicauta (Epicauta) ocellata (Dugès, 1869)}

Cantharis ocellata Dugès, 1869c: 160 (*)

TERRA TYPICA: “...hacienda de la Noria, á cinco leguas de la Piedad, en el Estado de Michoacan" originalmente; "Atlixco, Mexico..." tras designación de neotipo por Pinto (1980) en la colección de la California Academy of Sciences (San Francisco, EEUU) (fide Pinto, 1991).

Epicauta ocellata (Dugès, 1869): Dugès, 1886a: 582 (*)

COMENTARIOS TAXONÓMICOS: Especie incluida en el grupo de E. maculata por Pinto (1980, 1991). Horn (1885) y Beauregard (1890) consideraron a C. ocellata como sinónimo de E. maculata.

Distribución GeOgRÁfiCA: Especie endémica de México distribuida desde Coahuila hasta Oaxaca (Pinto, 1991). Citada en los estados de Coahuila, Durango, Michoacán, Morelos, Nayarit, Oaxaca, Puebla, San Luis Potosí y Tlaxcala. Dugès (1889e) y Zaragoza-Caballero (1999) señalan además México.

MÉxICo: Coahuila: $21 \mathrm{~km}$ al SE de Saltillo (Pinto, 1980, 1991); Torreón (Pinto, 1980, 1991). Durango: $20.4 \mathrm{mi}$. al NE de El Salto (Pinto, 1991); Villa (Ciudad) Lerdo (Pinto, 1980, 1991). Mexico: (Dugès, 1889e; Zaragoza-Caballero, 1999). Michoacán: Hacienda de la Noria, á cinco leguas de la Piedad (Dugès, 1869c; Champion, 1892). Morelos: Yautepec (Pinto, 1980, 1991). Nayarit: La Mesa de Nayar (Pinto, 1991). Oaxaca: Mitla (Pinto, 1980, 1991); $12 \mathrm{mi}$. al SE de Oaxaca (Pinto, 1991); Valerio Trujano (Pinto, 1980). Puebla: Atlixco (Pinto, 1980, 1991); $1 \mathrm{~km}$ al N de Chachapa (Pinto, 1980, 1991); Izúcar de Matamoros (Champion, 1892; Pinto, 1980, 1991); Lago Totolzingo (Pinto, 1991); Lago Valsequillo (Pinto, 1991); 14 km al NE de Cañada Morelos (Pinto, 1991); Puebla (Champion, 1892; Pinto, 1980, 1991); Tecamachalco (Pinto, 1980, 1991); $8 \mathrm{~km}$ al S de Tecamachalco (Pinto, 1980, 1991); 2 mi. al NO de Tehuacán (Pinto, 1991). San Luis Potosí: Ciudad del Maíz (Pinto, 1980, 1991); 15 km al E de San Luis Potosí (Pinto, 1980, 1991). Tlaxcala: $8 \mathrm{mi}$. al O de Apizaco (Pinto, 1991); $21 \mathrm{mi}$. ONO Apizaco (Pinto, 1991); $80 \mathrm{mi}$. al O de Xalapa (Pinto, 1991); 2 mi. al O de Tlaxcala (Pinto, 1991).

Epicauta (Epicauta) pardalis LeConte, 1866

Epicauta pardalis LeConte, 1866: $157(*)$

TERRA TYPICA: "New Mexico and Arizona" en la descripción original; "Valley of the Gila", tras designación de lectotipo. Lectotipo $\mathrm{n}^{\mathrm{o}}$ 4999, designado por Werner (1945) en la colección del Museum of Comparative Zoology (Harvard University, Cambridge, EEUU).

Comentarios taxonómicos: Especie incluida en el grupo de E. maculata por Pinto (1980, 1991). Esta especie, descrita originalmente como una posible raza de $E$. maculata, fue tratada por Horn (1873) y por Champion (1892) como una especie independiente, tal como se considera en la actualidad.

DISTRIBUCIÓN GEOGRÁFICA: Se distribuye por el noroeste de México y el suroeste de los EEUU (Pinto, 1980, 1991). Citada en los estados de Aguascalientes, Chihuahua, Coahuila, Durango y Sonora.

MÉXICO: Aguascalientes: $5 \mathrm{mi}$. al N de Rincón de Romos (sic) (Pinto, 1980). Chihuahua: $20 \mathrm{mi}$. al SO de Camargo (Vaurie, 1950; Pinto, 1980); 25 mi. al SO de Camargo (Vaurie, 1950; Pinto, 1980); 42 mi. al SO de Camargo (Pinto, 1980); 80 km al N de Chihuahua (Vaurie, 1950; Pinto, 1980); 92 km al N de Chihuahua (Vaurie, 1950); $5 \mathrm{mi}$. al S de Galeana (Pinto, 1980); $7 \mathrm{~km}$ al N de Jiménez (Pinto, 1980); Primavera (Vaurie, 1950; Pinto, 1980); Ojo Laguna (Vaurie, 1950; Pinto, 1980); $54 \mathrm{mi}$. al S de Sueco (Selander, 1954b); San José Babicora (Pinto, 1980); $10 \mathrm{mi}$. al S de Villa Ahumada (Pinto, 1980). Coahuila: Saltillo (Werner, 1945; Vaurie, 1950; Werner et al., 1966; Pinto, 1980). Durango: Descubridora (Werner, 1945; Vaurie, 1950; Pinto, 1980); cerca de Durango (Pinto, 1980); 40 mi. al S de Torreón (Pinto, 1980). Sonora: Nogales (Pinto, 1980).

\section{Epicauta (Epicauta) pedalis LeConte, 1866}

Epicauta pedalis LeConte, 1866: 157 (*)

TERRA TYPICA: "Cape San Lucas, Lower California". Lectotipo $\mathrm{n}^{\circ}$ 4997, designado por Werner (1945) en la colección del Museum of Comparative Zoology (Harvard University, Cambridge, EEUU) (fide Pinto, 1991).

COMENTARIOS TAXONÓMICOS: Especie incluida en el grupo de E. cupraeola por Pinto (1991). 
DisTRIBUCIÓN GEOGRÁFICA: Especie endémica de la Península de Baja California, sólo conocida en Baja California Sur (Pinto, 1991).

MÉxICo: Baja California Sur: Bahía Concepción (Pinto, 1991); Cabo San Lucas (Horn, 1894; Pinto, 1991); 4 km al NE de El Sauzal (Pinto, 1991); La Paz (Pinto, 1991); Miraflores (Pinto, 1991); 5 mi. al S de Miraflores (Pinto, 1991); 9.2 mi. al SSE de Mulegé (Pinto, 1991); 4 mi. al O de San Bartolo (Pinto, 1991); San José del Cabo (Pinto, 1991); 10 mi. al SO de San José del Cabo (Pinto, 1991); $10 \mathrm{~km}$ al S de San Pedro (Pinto, 1991); 6 mi. al SO de Santiago (Pinto, 1991); Sierra Giganta, $1 \mathrm{~km}$ al SO de Huatamote (Pinto, 1991); Sierra de San Lázaro (Pinto, 1991); Triunfo (Pinto, 1991); 6 mi. al N de Triunfo (Pinto, 1991).

\section{Epicauta (Epicauta) pensylvanica (De Geer, 1775)}

Cantharis (pensylvanica) De Geer, 1775: $16\left(^{*}\right)$

TERRA TYPICA: “...Pensylvanie”. Holotipo probablemente en la colección del Naturhistoriska Riksmuseet de Estocolmo (fide Pinto, 1991).

Lytta pensylvanica (De Geer, 1775): Gmelin in Linnaeus, 1789: 2016 (*) Meloe pensylvanica (De Geer, 1775): Gmelin in Linnaeus, 1789: 2020 (*) Meloe nigra Woodhouse, 1800: $213(*)$

TERRA TYPICA: no indicada. Pinto (1991) sugiere Pennsylvania, pero el tipo está aparentemente perdido (fide Pinto, 1991).

Lytta morio LeConte, 1854a: 447 (*)

TERRA TYPICA: "Texas", tras designación de lectotipo. Lectotipo $\mathrm{n}^{\circ}$ 5098 de la colección del Museum of Comparative Zoology (Harvard University, Cambridge, EEUU) designado por Werner (1945).

Epicauta pennsylvanica (De Geer, 1775): LeConte, 1866: 157 (enmienda injustificada) $(*)$

Epicauta pensylvanica (De Geer, 1775): Horn, 1873: $102\left(^{*}\right)$

Epicauta potosina Dugès, 1889e: 89 (*)

TERRA TYPICA: "Huasteca de St. Luis Potosi". Holotipo no 2295 (IBUNAM 029), en la colección del Instituto de Biología de la Universidad Nacional Autónoma de México (fide Vázquez \& Zaragoza, 1979; Pinto, 1991).

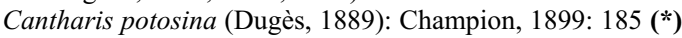

COMENTARIOS TAXONÓMICOS: Especie incluida en el grupo de E. cinerea por Pinto (1991). LeConte (1853), Horn (1873), Beauregard (1890), Champion (1892) y Borchmann (1917) incluyeron en su sinonimia además a E. atrata y E. coracina, pero Pinto (1991) no sigue este criterio y considera a $E$. atrata como una especie válida. Gmelin (1789) incluye a la especie de De Geer simultáneamente en los géneros Lytta y Meloe.

DISTRIBUCIÓN GEOGRÁFICA: Especie de amplia distribución, extendida desde San Luis Potosí hasta el sur del Canadá, con numeroros registros en el centro y este de los EEUU (Pinto, 1991). Existen dos ejemplares colectados en Jamaica (Gowdey, 1926), posiblemente producto de una introducción accidental, ya que la especie no se ha vuelto a encontrar en la isla (Selander \& Bouseman, 1960). En México se ha citado en los estados de Chihuahua, Coahuila, Durango, Nuevo León y San Luis Potosí.

MÉxICo: Chihuahua: $6 \mathrm{~km}$ al NE de Meoqui (Pinto, 1991); Salaices (Pinto, 1991). Coahuila: $61 \mathrm{mi}$. al N de Saltillo (Pinto, 1991); Monclova (Champion, 1892; Vaurie, 1950). Durango: Villa (Ciudad) Lerdo (Champion, 1892; Vaurie,
1950; Pinto, 1991; Zaragoza-Caballero, 1999 sub E. potosina); Yermo (Pinto, 1991). Nuevo León: Linares (Pinto, 1991); Monterrey (Pinto, 1991). San Luis Potosí: Huasteca Potosina (Dugès, 1889e sub E. potosina; Champion, 1892; Vaurie, 1950; Vázquez \& Zaragoza, 1979 sub E. potosina; Pinto, 1991; Zaragoza-Caballero, 1999 sub E. potosina).

\section{Epicauta (Epicauta) phoenix Werner, 1944}

Epicauta senilis Werner, 1944: $66\left(^{*}\right)$

TERRA TYPICA: "Phoenix, Arizona". Holotipo no 26070 de la colección del Museum of Comparative Zoology (Harvard University, Cambridge, EEUU) (fide Werner, 1944; Pinto, 1991).

COMENTARIOS TAXONÓMICOS: Especie incluida en el grupo de E. maculata por Pinto $(1980,1991)$.

Distribución geogrÁfica: Conocida de la Península de Baja California y del suroeste los EEUU (Pinto, 1980, 1991). En México sólo se ha citado en Baja California.

MÉXICO: Baja California: Algodones (Radford, 1960; Pinto, 1980).

Epicauta (Epicauta) puncticollis Mannerheim, 1843

Epicauta puncticollis Mannerheim, 1843: 288 (*)

TERRA TYPICA: "California"; Pinto (1991) indica que en el tipo se lee "Calif. bor.". Holotipo $\mathrm{n}^{\circ} 1330$ de la colección del Museum Zoologicum Universitatis (Helsinki) (fide Pinto, 1991).

Epicauta oblita LeConte, 1851: 162 (*)

TERRA TYPICA: "in Oregon et ad San Francisco" originalmente; "San Francisco, California", tras designación de lectotipo (fide Werner, 1945; Pinto, 1991). Lectotipo $n^{\circ} 4993$, designado por Werner (1945) en la colección del Museum of Comparative Zoology (Harvard University, Cambridge, EEUU)

Lytta puncticollis (Mannerheim, 1843): LeConte, 1853: 338 (*)

Lytta oblita (LeConte, 1851): LeConte, 1853: 339 (*)

Epicauta barberi Werner, 1944: 69 (*)

TERRA TYPICA: "La Panza, California". Holotipo no 4012 de la colección del United States National Museum of Natural History (Washington D.C.) (fide Pinto, 1991).

COMENTARIOS TAXONÓMICOS: Especie incluida en el grupo de E. sericans por Pinto (1991). Beauregard (1890) seguido por Borchmann (1917) trataron a E. oblita como especie independiente.

Distribución GeOgRÁfica: Conocida desde la Península de Baja California hasta el suroeste del Canadá, con numerosos registros en el oeste de los EEUU (Ballmer, 1979; Pinto, 1991). En México sólo se ha citado en Baja California.

MÉXICo: Baja California: El Sauzi [sic] (Ballmer, 1979); Ensenada (Ballmer, 1979); San Antonio del Mar (Ballmer, 1979); Santo Tomás (Ballmer, 1979).

\section{Epicauta (Epicauta) rileyi Horn, 1874}

Epicauta rileyi Horn, 1874: 37 (*)

TERRA TYPICA: "Arizona”. Lectotipo $\mathrm{n}^{\circ}$ 8095, designado por Werner (1945), en la colección del Museum of Comparative Zoology (Harvard University, Cambridge, EEUU).

Cantharis rileyi (Horn, 1874): Champion, 1899: 186 (*)

COMENTARIOS TAXONÓMICOS: Especie incluida en el grupo de E. caviceps tanto por Werner (1955) como por Pinto (1991). 
DISTRIBUCIÓN GEOGRÁFICA: Su distribución se limita al Estado de Sonora en México y al de Arizona en EEUU (Pinto, 1991).

MÉXICo: Sonora: Arispe (Werner, 1945; Pinto, 1991); Nogales (Pinto, 1991).

Epicauta (Epicauta) rufipennis (Chevrolat, 1834)

Lytta rufipennis Chevrolat, $1834 \mathrm{~b}$ : fasc. $4, \mathrm{n}^{\circ} 80$ (sin paginar) (*)

TERRA TYPICA: "environs de Mexico". Holotipo posiblemente en la colección del Museum Zoologicum Universitatis (Helsinki) (fide Pinto, 1991)

Cantharis rufipennis (Chevrolat, 1834): Dugès, 1869b: 127 (*)

Cantharis ocreaceipennis Dugès, 1870: 164 (*)

TERRA TYPICA: “...cercanías de Guanajuato”. Tipo aparentemente perdido (fide Pinto, 1991)

Cantharis ochreaceipennis Dugès, 1870: Dugès, 1870: 169 (enmienda justificada) (*)

Cantharis ochreipennis Dugès, 1877: 57 (*)

TERRA TYPICA: "Estado de Veracruz,... mineral de la Luz á algunas leguas de Guanajuato;...en uno de los paseos de esta última ciudad". Tipo aparentemente perdido (fide Pinto, 1991).

Epicauta rufipennis (Chevrolat, 1834): Dugès, 1886a: $582(*)$

COMENTARIOS TAXONÓMICOS: La atribución genérica del nombre Lytta rufipennis ha generado cierta confusión debido a una equivocación de Crotch (1873), quien incluyó implícitamente a $C$. rufipennis en el género Pyrota por un error de sinonimia. Este error ya fue criticado por Horn (1885: 114), pero aún así Borchmann (1917), Denier (1935a) y Blackwelder (1945) continuaron incluyendo el nombre inexistente Pyrota rufipennis (Chevrolat) como sinónimo de Pyrota dubitabilis o de $P$. tenuicostatis.

Los nombres no disponibles Epicauta ochreipennis Sturm, 1843: 174 y Epicauta luridipennis Deyrolle fueron recogidos por Dugès (1877) en la sinonimia de $C$. ochreipennis; y el de E. luridipennis Deyrolle por Dugès (1889e) en la sinonimia de C. rufipennis. Champion (1892, 1899) señala los nombres no disponibles Epicauta frontalis Sturm, 1843: 174 y Epicauta luridipennis Dejean, 1837: 247, atribuibles a esta especie; mientras que Beauregard (1890) considera especie válida a Epicauta luridipennis y atribuye su autoría a Dugès.

Especie incluida en el grupo de E. cupraeola por Pinto (1991). Pinto (1991) indica que los ejemplares de Jalisco, Nayarit y Chihuahua difieren del resto en la pilosidad.

DISTRIBUCIÓN GEOGRÁFICA: Especie presente en México y Guatemala (Pinto, 1991). En México se ha citado en los estados de Chihuahua, Distrito Federal, Guanajuato, Guerrero, Hidalgo, Jalisco, México, Michoacán, Morelos, Nayarit, Oaxaca, Puebla, Sinaloa y Veracruz.

MÉxICo: Chihuahua: Santo Niño (Pinto, 1991); 4 mi. al SO de Temores (Pinto, 1991). Distrito Federal: Ixtapalapa (Pinto, 1991); Ciudad de México (Chevrolat, 1834b; Dugès, 1869b; Champion, 1892; Pinto, 1991). Guanajuato: $22 \mathrm{mi}$. al NE de Guanajuato (Pinto, 1991); Guanajuato (Dugès, 1870;
Dugès, 1877 sub C. ochreipennis; Dugès, 1889e; Champion, 1892 sub C. ochraceipennis); Mineral de la Luz (Dugès, 1877 sub C. ochreipennis; Champion, 1892 sub C. ochraceipennis). Guerrero: Chilpancingo (Champion, 1892; Pinto, 1991); 13 mi. al $\mathrm{N}$ de Chilpancingo (Pinto, 1991); $20 \mathrm{mi}$. al $\mathrm{N}$ de Chilpancingo (Pinto, 1991); $24 \mathrm{mi}$. al $\mathrm{N}$ de Chilpancingo (Pinto, 1991); $32 \mathrm{~km}$ al O de Iguala (Pinto, 1991); Mexcala (Champion, 1892; Pinto, 1991); Omilteme (Champion, 1892). Hidalgo: Jacala (Pinto, 1991); Pachuca (Pinto, 1991); Tepeapulco (Pinto, 1991). Jalisco: $25 \mathrm{~km}$ al O de Magdalena (Pinto, 1991); $10 \mathrm{~km}$ al O de Tequila (Pinto, 1991). México: Real de Arriba (Pinto, 1991); Tejupilco (Pinto, 1991); Temescaltepec (Pinto, 1991); $1 \mathrm{mi}$. al E de Temescaltepec (Pinto, 1991). Michoacán: $5 \mathrm{~km}$ al O de Ciudad Hidalgo (Pinto, 1991); $3 \mathrm{~km}$ al E de Quiroga (Pinto, 1991); Uruápan (Pinto, 1991). Morelos: Cuernavaca (Pinto, 1991); Tepoztlán (Pinto, 1991). Nayarit: 7 mi. al NO de Tepic (Pinto, 1991); 24 mi. al SE de Tepic (Pinto, 1991). Oaxaca: Monte Albán (Pinto, 1991); Oaxaca (Champion, 1892); $16 \mathrm{mi}$. al NO de Oaxaca (Pinto, 1991); Tehuantepec (Champion, 1892); Yolotepec (Champion, 1892). Puebla: 9 mi. al S de Izúcar de Matamoros (Pinto, 1991). Sinaloa: Santa Lucía (Pinto, 1991). Veracruz: Xalapa (Champion, 1892; Pinto, 1991); Veracruz (Dugès, $1889 \mathrm{e})$.

Epicauta (Epicauta) senilis Werner, 1949

Epicauta senilis Werner, 1949b: 102 (*)

TERRA TYPICA: "Luna Co., New Mexico, 4000 ft.". Aunque Werner (1949b) indica que el tipo está en la colección de la Academy of Natural Sciences de Philadelphia, pero Pinto (1991) señala que allí no se encuentra y que, por lo tanto, está perdido.

COMENTARIOS TAXONÓMICOS: Especie incluida en el grupo de E. caviceps por Pinto (1991).

DistribuCIÓN GEOGRÁFICA: Conocida del norte de México y del extremo centro-meridional de los EEUU (Pinto, 1991). Se ha citado en Chihuahua y Durango. Pinto (1991) indica que Dillon (1952) citó la presencia de E. senilis en algunas localidades bajo la denominación de $E$. candidata.

MÉxICo: Chihuahua: Ojo de Laguna (25 mi. al S de Gallego) (Pinto, 1991). Durango: $9 \mathrm{mi}$. al O de Durango (Pinto, 1991); 1 mi. al N de Registro (Pinto, 1991); 5 mi. al NE de Sauz (Pinto, 1991).

\section{Epicauta (Epicauta) sericans LeConte, 1866}

Epicauta sericans LeConte, 1866: 158 (*)

TERRA TYPICA: "Kansas, New Mexico, and Texas", en la descripción original; "Kansas", tras designación de lectotipo ( $\mathrm{n}^{\circ}$ 4994) por Werner (1945) en la colección del Museum of Comparative Zoology (Harvard University, Cambridge, EEUU).

Lytta sartorii Haag-Rutenberg, 1880: 56 (*)

TerRa TYPICA: "Mexico, Mirador". Según Pinto (1991) los tipos no están en los museos donde se encuentran otros tipos de HaagRutenberg.

Epicauta sartorii (Haag-Rutenberg, 1880): Beauregard, 1890: 510 (*) Cantharis sartorii (Haag-Rutenberg, 1880): Champion, 1899: 187 (*)

COMEnTARIOS TAXONÓMICOS: Especie incluida en el grupo de E. sericans por Pinto (1991). Pinto (1991) establece tentativamente la sinonimia de $L$. sartorii con $E$. sericans. Vaurie (1950) indica que existe variabilidad 
geográfica en el color de la pilosidad, y que ésta podría corresponder a la existencia de subespecies diferentes. Horn (1873) y Beauregard (1890) incluyen con dudas a C. immerita Walker, 1866, en la sinonimia de E. sericans; Borchmann (1917: 82) también incluye a C. immerita en la sinonimia de E. sericans (pero en el mismo catálogo la incluye en el género Lytta, Borchmann, 1917: 95), mientras que Pinto (1991) no lo considera así. Werner et al. (1966) indican que los ejemplares de la meseta central mexicana podrían corresponder a una especie diferente.

DisTRIBUCIÓN GEOGRÁFICA: Especie ampliamente extendida, desde la región centro-oriental de México hasta el sur del Canadá, con numerosas citas en los EEUU (Pinto, 1991). En México se ha citado en los estados de Chihuahua, Coahuila, Distrito Federal, Durango, Hidalgo, Jalisco, México, Nuevo León, Puebla, Querétaro, San Luis Potosí, Tamaulipas y Veracruz. Pinto (1991) no ha examinado todos los ejemplares correspondientes a las citas de E. ferruginea de Vaurie (1950), por lo que alguna podría corresponder a E. sericans (sensu Pinto, 1991). Pinto (1991) indica que la descripción de la larva de E. callosa de MacSwain (1956) corresponde a $E$. sericans.

MÉxICo: Chihuahua: 2 mi. al S de Metachic (Pinto, 1991); 2 mi. al O de Pedernales (Pinto, 1991); Salaices (Pinto, 1991); 6 mi. al S de Villa Matamoros (Pinto, 1991); Madera (Vaurie, 1950). Coahuila: $20 \mathrm{mi}$. al N de Monclova (Pinto, 1991); 1 mi. al SE de Saltillo (Pinto, 1991); 17 mi. al SE de Saltillo (Pinto, 1991); Mesa de la Encantada, Sierra del Carmen (Pinto, 1991); 22 mi. al N de Zaragoza (Pinto, 1991); $192 \mathrm{~km}$ al S de Piedras Negras (Vaurie, 1950). Distrito Federal: Guadalupe (Vaurie, 1950). Durango: 20-22 mi. al NE de Durango (Pinto, 1991); El Salto (Pinto, 1991); $28 \mathrm{mi}$. al E de El Salto (Pinto, 1991); 6 mi. al E de El Salto (Pinto, 1991); 6 mi. al NE de El Salto (Vaurie, 1950); 5 mi. al NE de El Sauz (Pinto, 1991); Palos Colorados (Vaurie, 1950; Pinto, 1991); 12 mi. al SO de Villa Madero (Pinto, 1991). Hidalgo: Chapulhuacán (Pinto, 1991); Zimapán (Pinto, 1991). Jalisco: Guadalajara (Pinto, 1991); Poncitlán (Pinto, 1991). México: Teotihuacán (Pinto, 1991); $22 \mathrm{mi}$. al $\mathrm{N}$ de Toluca (Pinto, 1991). Nuevo León: 7 mi. al NE de Allende (Pinto, 1991); Apodaca (Pinto, 1991); Campo Apodaca (Pinto, 1991); Cerro de La Silla (Pinto, 1991); 12 mi. al O de China (Pinto, 1991); Mesa Chipinque (Pinto, 1991); $5 \mathrm{mi}$. al O de Doctor Arroyo (Pinto, 1991); El Carmen (Pinto, 1991); 4 mi. al O de El Cercado (Pinto, 1991); 11 mi. al O de Galeana (Pinto, 1991); 16 mi. al N de La Gloria (Pinto, 1991); Linares (Pinto, 1991); 4-15 mi. al O de Linares (Pinto, 1991); 16 mi. al S de Linares (Pinto, 1991); Montemorelos (Pinto, 1991); $9 \mathrm{~km}$ al O de Montemorelos (Pinto, 1991); Monterrey (Pinto, 1991); $10 \mathrm{mi}$. al N de Providencia (Pinto, 1991); 15 mi. al NE de Sabinas (Pinto, 1991); Sabinas Hidalgo (Pinto, 1991); Salinas Victoria (Pinto, 1991); 41 mi. al S de Saltillo (Pinto, 1991); Río San Juan (Pinto, 1991); Vallecillo (Pinto, 1991); Hacienda Vista Hermosa, Villa Santiago (Pinto, 1991). Puebla: Gilberto Comacho [sic] (Pinto, 1991); Lago Totoltzingo (Pinto, 1991); $5.1 \mathrm{mi}$. al SO de Tehuacán (Pinto, 1991); Zacatepec (Pinto,
1991); 4.5 mi. al O de Zacatepec (Pinto, 1991). Querétaro: 1 mi. al NO de Ayutla (Pinto, 1991); 5 mi. al NO de Jalpan (Pinto, 1991). San Luis Potosí: 3 mi. al O de El Cedral (Pinto, 1991); 6-13 mi. al E de Ciudad del Maíz (Pinto, 1991); 5 mi. NE de Ciudad del Maíz (Pinto, 1991); El Banito (Pinto, 1991); El Naranjo (Pinto, 1991); 1-2 mi. al E del Naranjo (Pinto, 1991); Cascada de El Salto (Pinto, 1991); 3 mi. al E de la Cascada de El Salto (Pinto, 1991); $20 \mathrm{mi}$. al S de Huichihuayan (Pinto, 1991); $6 \mathrm{mi}$. al S de Matalpa (Pinto, 1991); Picolo (Pinto, 1991); Pujal (Pinto, 1991); Tamazunchale (Pinto, 1991); 6-25 mi. al N de Tamazunchale (Pinto, 1991); 15 mi. al NO de Tamazunchale (Pinto, 1991); $10 \mathrm{mi}$. al SO de Tamazunchale (Pinto, 1991); $18 \mathrm{mi}$. al S de Tamazunchale (Pinto, 1991); Tamuín (Pinto, 1991); 14 mi. al NE de Tamuín (Pinto, 1991); Valles (Vaurie, 1950; Pinto, 1991); 22 mi. al N de Valles (Pinto, 1991); Xilitla (Pinto, 1991); 2 mi. al E de Xilitla (Pinto, 1991). Tamaulipas: Ciudad Victoria (Pinto, 1991); $19 \mathrm{mi}$. al S de Ciudad Victoria (Pinto, 1991); 62 mi. al NE de Ciudad Victoria (Pinto, 1991); 20 mi. al N de El Limón (Pinto, 1991); 24 km al E de El Mante (Pinto, 1991); 45 mi. al E de El Mante (Pinto, 1991); 2 mi. al SE de Gómez Farías (Pinto, 1991); 7 km al SSE de Gómez Farías (Pinto, 1991); 15 mi. al NE de Juamave (Pinto, 1991); 5.7 mi. al N de La Encarnación (Pinto, 1991); 5 mi. al N de Llera (Pinto, 1991); Manuel (Pinto, 1991); $40 \mathrm{mi}$. al S de Nuevo Laredo (Pinto, 1991); Nuevo Morelos (Pinto, 1991); 1 mi. al E de Nuevo Morelos (Pinto, 1991); Ocampo (Pinto, 1991); Cañón del Peregrino (Pinto, 1991); Río Sabinas (Pinto, 1991); !6 km al O de San Fernando (Pinto, 1991); 10 mi. al E de Soto la Marina (Pinto, 1991); $40 \mathrm{~km}$ al N de Soto la Marina (Pinto, 1991); Villagrán (Pinto, 1991); 9 mi. al S de Villagrán (Pinto, 1991); $14 \mathrm{~km}$ al S de Villagrán (Pinto, 1991). Veracruz: 7 mi. al NO de Alazán (Pinto, 1991); Camarancillo (Pinto, 1991); Camarillo, $24 \mathrm{~km}$ al SO de Conejos (Pinto, 1991); El Tajín (Pinto, 1991); Jicaltepec (Pinto, 1991); 6 mi. al SE de Rinconada (Pinto, 1991); 4 mi. al NO de Rinconada Antigua (Pinto, 1991); $10 \mathrm{mi}$. al O de Tampico (Pinto, 1991); 29 mi. al SE de Tantoyuca (Pinto, 1991); $1 \mathrm{mi}$. al N de Tecolutla (Pinto, 1991); 9 mi. al N de Tempoal (Pinto, 1991); Tuxpan (Pinto, 1991); 9 mi. al S de Tuxpan (Pinto, 1991); 20 mi. al SO de Veracruz (Pinto, 1991); Hacienda El Mirador (Haag-Rutenberg, 1880 sub L. sartorii; Vaurie, 1950 sub. E. ferruginea).

\section{Epicauta (Epicauta) singularis Champion, 1892}

Epicauta singularis Champion, 1892: 427 (*)

Terra tyPica: "MeXico, Monterey in Nuevo Leon, Saltillo in Coahuila..." originalmente; "Monterey, Nuevo Leon..." tras designación de lectotipo (Pinto, 1982) en la colección del British Museum of Natural History (Londres).

Cantharis singularis (Champion, 1892): Champion, 1899: 188 (*)

COMENTARIOS TAXONÓMICOS: Especie incluida en el grupo de E. caviceps por Werner (1955) y Pinto (1991).

DisTRIBUCIÓN GEOGRÁFICA: Distribuida exclusivamente por el noreste de México y el sureste de los EEUU (Pinto, 1991). En México se ha citado en Coahuila y Nuevo León.

MÉxICO: Coahuila: Saltillo (Champion, 1892; Vaurie, 1950; Werner, 1955). Nuevo León: 3 mi. al N de la Cumbre de Mamulique (Pinto, 1991); Monterrery (Champion, 1892; 
Vaurie, 1950; Werner, 1955; Pinto, 1982, 1991); 15 mi. al O de Monterrey (Pinto, 1991); $7 \mathrm{~km}$ al O de Santa Catarina (Pinto, 1991); Villa de García (Pinto, 1991).

\section{Epicauta (Epicauta) straba Horn, 1891}

Epicauta straba Horn, 1891: 42 (*)

TeRrA TYPICA: "San Bernardino, Cal.". Lectotipo no 8096, designado por Werner (1945), inicialmente en la colección de la Academy of Natural Sciences of Philadelphia, posteriormente transferido al Museum of Comparative Zoology de Harvard (EEUU)

Cantharis straba (Horn, 1891): Champion, 1899: 188 (*)

Epicauta foxi Van Dyke, 1929: 127 (*)

TERRA TYPICA: "Jacumba, San Diego Co., Calif.". Holotipo no 2600 , en la colección de la California Academy of Sciences (San Francisco, EEUU) (fide Van Dyke, 1929; Pinto, 1991).

COMENTARIOS TAXONÓMICOS: Especie incluida en el grupo de E. caviceps por Werner (1955) y Pinto (1991).

DISTRIBUCIÓN GEOGRÁFICA: Su distribución se limita al

Estado de Baja California en México y al de California en EEUU (Pinto, 1991).

MÉxICo: Baja California: $3.3 \mathrm{mi}$. al S de El Condor (Pinto, 1991)

\section{Epicauta (Epicauta) subatra Dugès, 1889}

Epicauta subatra Dugès, 1889e: 72 (*)

TERRA TYPICA: "Mexico" originalmente; "Hacienda Vista Hermosa Villa Santiago, Nuevo León;... 1500'..." tras designación como neotipo de un ejemplar de la colección del United States National Museum of Natural History (Washington D.C.) (Pinto, 1991). Cantharis subatra (Dugès, 1889): Champion, 1899: 188 (*)

COMENTARIOS TAXONÓMICOS: Especie incluida en el grupo de E. cinerea por Pinto (1991). Pinto (1991) indica que es una especie con variación geográfica en la coloración de la pilosidad y en las dimensiones del pronoto. Dugès (1886a: 582) utiliza por primera vez el nombre E. subatra sin acompañarlo de una descripción.

DistribuCión GEOGRÁFICA: Especie endémica de México (Pinto, 1991). Citada en el Distrito Federal, Hidalgo, Nuevo León y Puebla. Según Pinto (1991), las citas de México esta especie de Denier (1935a,b) corresponden a $E$. major. Citada erróneamente de Nicaragua (Blackwelder, 1945).

MÉxICo: Distrito Federal: México (Dugès, 1889e). Hidalgo: Molinos de Guerrero (Guerrero) (Pinto, 1991); $8 \mathrm{mi}$. al NE de Jacala (Pinto, 1991); $10 \mathrm{mi}$. al NE de Jacala (Pinto, 1991). Nuevo León: Cerro Potosí (Pinto, 1991); Mesa Chipinque cerca de Monterrey (Pinto, 1991); Cascada Cola de Caballo (Pinto, 1991); Hacienda Vista Hermosa, Villa Santiago (Pinto, 1991); Las Adjuntas, cerca de Villa de Santiago (Pinto, 1991). Puebla: 6 mi. al O de Teziutlán (Pinto, 1991).

\footnotetext{
Epicauta (Epicauta) subrubra Dugès, 1889

Epicauta subrubra Dugès, 1889e: 83 (*)

TERRA TYPICA: "Mexico". El tipo, único ejemplar conocido de la especie, está aparentemente perdido (fide Pinto, 1991). ZaragozaCaballero (1999) señala que según un manuscrito inédito de Dugès el ejemplar tendría el número 2211.

Cantharis subrubra (Dugès, 1889): Champion, 1899: 188 (*)
}

Comentarios taxonómicos: Pinto (1991) indica que se trata de un "nomen dubium", ya que el tipo se ha perdido y no es posible aplicar el nombre a ninguna especie conocida. Sin embargo, la descripción original es válida aunque inadecuada y, según Pinto (1991), parecería acercarse a las especies del grupo de E. caustica. Dugès (1886a: 582) usó previamente el mismo nombre sin acompañarlo de una descripción, creando un nombre no disponible.

Distribución GEOGRÁficA: Especie descrita de México, sin mayor precisión (aunque posiblemente se trate del Distrito Federal), de la que actualmente no se conoce ningún ejemplar en existencia (Pinto, 1991).

\section{Epicauta (Epicauta) tarasca Pinto, 1991}

Epicauta tarasca Pinto, 1991: 207 (*)

TERRA TYPICA: "3 km E Quiroga, Michoacán, Mexico". Holotipo en la colección de la California Academy of Sciences (San Francisco, EEUU) (fide Pinto, 1991).

COMENTARIOS TAXONÓMICOS: Especie incluida en el grupo de E. cinerea por Pinto (1991).

DisTRIBUCIÓN GEOGRÁfICA: Endemismo mexicano (Pinto, 1991). Citado en los estados de Jalisco, Michoacán, Morelos y Oaxaca

MÉxICo: Jalisco: Colimilla, Barranca de Oblatos (Pinto, 1991). Michoacán: 6 mi. al S de Carapan (Pinto, 1991); Cerro Tancítaro (Pinto, 1991); Lago de Pátzcuaro (Pinto, 1991); 16.4 mi. al E de Morelia (Pinto, 1991); Quiroga (Pinto, 1991); 3 km al E de Quiroga (Pinto, 1991). Morelos: $15 \mathrm{mi}$. N. Cuernavaca (Pinto, 1991). Oaxaca: Cerro Pelón, en la carretera 175 (Pinto, 1991); 52 mi. al O de Oaxaca (Pinto, 1991); $10 \mathrm{mi}$. al E de Santos Reyes Pápalo (Pinto, 1991).

Epicauta (Epicauta) temexa Adams et Selander, 1979 Epicauta temexa Adams et Selander, 1979: 248 (*)

TERRA TYPICA: "Pearsall, Frio County, Texas...". Holotipo en la colección del American Museum of Natural History (EEUU) (fide Adams \& Selander, 1979; Pinto, 1991).

COMENTARIOS TAXONÓMICOS: Especie incluida en el grupo de E. vittata por Adams \& Selander (1979), Agafitei \& Selander (1980) y Pinto (1991).

Adams \& Selander (1979) y Pinto (1991) indican que Champion (1892), Blackwelder (1945) y Hernández (1960) se refirieren a esta especie como E. lemniscata (Fabricius, 1801), mientras que Dugès (1886a; 1889e) se refiere a ella con el nombre de E. vittata (Fabricius, 1775). Werner (1945) indica la presencia probable en México de E. lemniscata "extending far into Mexico". Según Adams \& Selander (1979) y Pinto (1991), ambos nombres (E. vittata y E. lemniscata) corresponden a subespecies de $E$. vittata, una especie que no se encuentra en México, donde está substituida por E. temexa. Un error tipográfico en la obra de Dugès (1886a: 582; ver Dugès, 1889e: 87) por el que se atribuye la especie Epicauta vittata al propio Dugès, generó la propuesta 
por Beauregard (1889: ccxiii) de un nombre de substitución inadecuado: E. dugesi Beauregard, 1889 (nombre no disponible según el artículo 12.2.3 del Código Internacional de Nomenclatura Zoológica, CINZ, 2000; ver comentarios al respecto de E. dugesi Werner, 1957), que Champion (1899: 177) volvió a usar con el nombre de Cantharis dugesi. Vázquez \& Zaragoza (1979) indican como tipo de E. vittata Dugès el ejemplar no 1889 (IBUNAM 028) de la colección del Instituto de Biología de la Universidad Nacional Autónoma de México, sin embargo este ejemplar no es un tipo, ya que no corresponde a ninguna especie descrita. MacSwain (1956) se refiere a esta especie como Epicauta sp. "a".

DISTRIBUCIÓN GEOGRÁFICA: Especie con una distribución restringida al este de México y extremo sureste de los EEUU (Pinto, 1991). Citado en los estados de Coahuila, Nuevo León, San Luis Potosí, Tamaulipas y Veracruz. Calvino et al. (1920) citan E. lemniscata del Distrito Federal, pero Adams \& Selander (1979), señalan que esa cita podría corresponder a $E$. temexa, aunque se inclinan a pensar que en realidad, la cita es de un ejemplar que no corresponde a ninguna especie del grupo de $E$. vittata.

MÉxICo: Coahuila: $12 \mathrm{mi}$. al $\mathrm{N}$ de Jiménez (Adams \& Selander, 1979); Las Rusias, Múzquiz (Adams \& Selander, 1979). Nuevo León: Apodaca (Adams \& Selander, 1979); Allende (Adams \& Selander, 1979); Linares (Adams \& Selander, 1979); Montemorelos (Adams \& Selander, 1979); Monterrey (Adams \& Selander, 1979); región de Monterrey (Hernández, 1960 sub E. lemniscata; Adams \& Selander, 1979); Sabinas Hidalgo (Adams \& Selander, 1979). San Luis Potosí: Ciudad Valles (Adams \& Selander, 1979); El Salto (Adams \& Selander, 1979); Guerrero (Adams \& Selander, 1979); Hacienda de "Santa Clara", Huasteca Potosina (Dugès, 1889e sub E. vittata; Adams \& Selander, 1979; Vázquez \& Zaragoza, 1979 sub E. vittata). Tamaulipas: Ciudad Mante (Adams \& Selander, 1979); Ciudad Victoria (Adams \& Selander, 1979); El Limón (Adams \& Selander, 1979); Nuevo Laredo (Adams \& Selander, 1979); Tampico (Adams \& Selander, 1979). Veracruz: Alazán (Adams \& Selander, 1979); El Naranjo (Adams \& Selander, 1979); 4 mi. al SO de Panuco (Adams \& Selander, 1979).

\section{Epicauta (Epicauta) tenebrosa Werner, 1949}

\section{Epicauta tenebrosa Werner, 1949b: 93 (*)}

TERRA TYPICA: "Tucson, Arizona". Holotipo no 28221, en la colección del Museum of Comparative Zoology (Harvard University, Cambridge, EEUU) (fide Werner, 1949b; Pinto, 1991).

COMENTARIOS TAXONÓMICOS: Especie incluida en el grupo de E. cupraeola por Pinto (1991).

DisTRIBUCIÓN GEOGRÁFICA: Repartida por el norte de Sonora y el sur de Arizona (Pinto, 1991). Pinto (1991) indica que Horn (1894) y Werner (1945) han citado ejemplares de EEUU de esta especie bajo la denominación de E. pedalis.

MÉxICO: Sonora: Bahía Kino (Pinto, 1991); Navajoa (Pinto, 1991).
Epicauta (Epicauta) teresa Mathieu, 1983

Epicauta (Epicauta) teresa Mathieu, 1983: 156 (*)

Terra tYPICA: "10 km NE of Cintalapa, Chiapas, Mexico". Holotipo, en la colección de la California Academy of Sciences (San Francisco, EEUU) (fide Mathieu, 1983; Pinto, 1991).

COMENTARIOS TAXONÓMICOS: Especie incluida en el grupo de E. curvicornis por Pinto (1991). Los ejemplares de Oaxaca parecen mostrar algunos rasgos diferenciales en la coloración de la pilosidad (Pinto, 1991).

DistribuCión GEOGRÁFICA: Especie endémica de México (Pinto, 1991) citada en Chiapas y Oaxaca.

MÉxICo: Chiapas: $10 \mathrm{~km}$ al NE de Cintalapa (Mathieu, 1983; Pinto, 1991); Cañón del Sumidero, El Sumidero (Pinto, 1991); $22 \mathrm{~km}$ al SO de Ocozocuautla (Pinto, 1991). Oaxaca: 19 mi. al S de Matías Romero (Pinto, 1991); 14 mi. al O de Niltepec (Pinto, 1991).

Epicauta (Epicauta) tricostata (Werner, 1943)

Pleuropompha tricostata Werner, 1943: 32 (*)

TERRA TYPICA: "Presidio, Texas". Holotipo n 56742, de la colección del United States National Museum of Natural History (Washington D.C.) (fide Werner, 1943; Pinto, 1991). La serie típica consta de 14 ejemplares más (Werner, 1943)

Epicauta tricostata (Werner, 1943): Pinto, 1984b: 231 (*)

COMENTARIOS TAXONÓMICOS: Especie incluida en el grupo de E. costata por Pinto (1991).

DistribuCión GEOGRÁFICA: Distribuida por el norte de México y el extremo sur de los EEUU (Pinto, 1991). En México se ha encontrado en los estados de Chihuahua, Coahuila y San Luis Potosí. Werner et al. (1966) indican que existe un registro, posiblemente accidental, en Oaxaca. Pinto (1991), tras examinar esos ejemplares de la colección de la California Academy of Sciences, duda de la prodecencia oaxaqueña de este material con etiqueta de: "Valerio Trujano, Oao" por lo que aquí no lo consideramos.

MÉxICo: Chihuahua: 42 mi. al SO de Camargo (Vaurie, 1950); Delicias (Vaurie, 1950). Coahuila: $20 \mathrm{mi}$. al N de Carranza (Pinto, 1991); Guadalupe (Vaurie, 1950; Pinto, 1991); 61 mi. al N de Saltillo (Pinto, 1991); Torreón (Pinto, 1991). San Luis Potosí: $46.9 \mathrm{~km}$ al NE de Villa Hidalgo (Pinto, 1991).

Epicauta (Epicauta) unilineata Champion, 1892

Epicauta unilineata Champion, 1892: 415 (*)

TeRra typica: "Guatemala, near de city 5000 feet, San Gerónimo", originalmente; "San Jerónimo, Guatemala" tras designación de lectotipo. Lectotipo designado por Adams \& Selander (1979) en la colección de la Academy of Natural Sciences of Philadelphia (EEUU), pero al parecer está perdido (fide Pinto, 1991).

Cantharis unilineata (Champion, 1892): Champion, 1899: 190 (*)

Comentarios taxonómicos: Especie incluida en el grupo de E. vittata por Adams \& Selander (1979), Agafitei \& Selander (1980) y Pinto (1991). Pinto (1991) indica que es muy probable que $E$. tamara sea una raza geográfica de E. unilineata, aunque no toma una decisión al respecto, ya que parecen existir diferencias entre las dos en el cortejo 
(Adams \& Selander, 1979). Werner (1957b) al hablar de $E$. tamara (sub E. dugesi) indica que el ejemplar examinado por él de Tehuantepec podría corresponder a una especie diferente. Pinto (1991) opina que esta cita correspondenría seguramente a $E$. unilineata .

DisTRIBUCIÓN GEOGRÁFICA: Su distribución se extiende por El Salvador, Guatemala y el sur de México (Pinto, 1991). En México se ha citado en Chiapas, Guerrero, Oaxaca, Puebla y Veracruz.

MÉxICo: Chiapas: Suchiapa (Adams \& Selander, 1979). Guerrero: $43.7 \mathrm{~km}$ al NO de Ixtapa (Pinto, 1991). Oaxaca: Juchitán (Adams \& Selander, 1979); Tehuantepec (Adams \& Selander, 1979). Puebla: Cacaloapan (Adams \& Selander, 1979). Veracruz: Estación Agrícola Experimental de Cotaxtla (Adams \& Selander, 1979; Agafitei \& Selander, 1980); La Granja (Adams \& Selander, 1979); $10 \mathrm{mi}$. al O de Tezonapa (Adams \& Selander, 1979); 5 mi. al NE de Tinajas (Adams \& Selander, 1979); Veracruz (Adams \& Selander, 1979).

Epicauta (Epicauta) vitticollis (Haag-Rutenberg, 1880) Lytta vitticollis Haag-Rutenberg, 1880: 52 (*)

TERRA TYPICA: "Nicaragua (St. Juan del Norte), Guatimala (sic), Mexico..." originalmente; "Guatimal..." tras designación de lectotipo (Pinto, 1991). Lectotipo designado por Pinto (1991) en la colección del Zoologische Staatssammlung (Munich). Paralectotipos de Nicaragua y de San Juan del Norte, Nicaragua en la misma colección (fide Pinto, 1991).

Epicauta canoi Dugès, 1889 e: $86(*)$

TERRA TYPICA: "Veracruz". Tipo al parecer perdido (fide Pinto, 1991).

Epicauta vitticollis (Haag-Rutenberg, 1880): Beauregard, 1890: $512(*)$ Cantharis vitticollis (Haag-Rutenberg, 1880): Champion, 1899: 191 (*)

COMENTARIOS TAXONÓMICOS: Especie incluida en el grupo de E. vittata por Adams \& Selander (1979), Agafitei \& Selander (1980) y Pinto (1991). HaagRutenberg (1880), Beauregard (1890) y Champion (1892) incluyen en la sinonimia los nombres no disponibles ruficrus Chevr. in litt. y confluens Deyr. in litt.

DisTRIBUCIÓN GEOGRÁfICA: Especie citada en Belice, Guatemala, Honduras, México y Nicaragua (Pinto, 1991; Maes \& Huether, 2007). En México se distribuye en los estados de Campeche, Chiapas, Oaxaca, Quintana Roo, Tabasco, Veracruz y Yucatán. En Tabasco causa daños a los cultivos de frijol (Sánchez Soto, 2001).

MÉxICo: Campeche: Ruinas Edzna (Pinto, 1991). Chiapas: Colonia Chupa, $5 \mathrm{mi}$. al S de Palenque (Adams \& Selander, 1979). Oaxaca: Donaji [sic] (Adams \& Selander, 1979); Tuxtepec (Adams \& Selander, 1979). Quintana Roo: 15 mi. al O de Chetumal (Adams \& Selander, 1979). Tabasco: $5 \mathrm{~km}$ al E de Cárdenas (Adams \& Selander, 1979); Frontera (Champion, 1892); Teapa (Champion, 1892); Tepas (Adams \& Selander, 1979); Villahermosa (Adams \& Selander, 1979). Veracruz: 2 mi. al O de Ciudad Alemán (Adams \& Selander, 1979); Córdoba (Adams \& Selander, 1979); Cosamaloapan (Champion, 1892; Adams \& Selander, 1979); Estación Agrícola Experimental de Cotaxtla (Adams \& Selander, 1979; Agafitei \& Selander, 1980); 31 km ONO El Potrero (Adams \& Selander, 1979); 10 mi. al O de Tezonapa (Adams \& Selander,
1979); Veracruz (Dugès, 1889e sub E. canoi; Champion, 1892; Adams \& Selander, 1979). Yucatán: Pisté (Adams \& Selander, 1979).

Subgénero Macrobasis LeConte, 1862

Macrobasis LeConte, 1862: 272 (*)

ESPECIE TIPO: Lytta albida Say, 1824, por designación subsecuente de Wellman (1910b).

Apterospasta LeConte, 1862: 272 (*)

ESPECIE TIPO: Lytta segmenta Say, 1824, por designación subsecuente de Wellman (1910b).

Gnathospasta Horn, 1875: 154 (*)

ESPECIE TIPO: Gnathospasta mimetica Horn, 1875, por monotipia.

CONTENIDO Y DISTRIBUCIÓN: El subgénero incluye 72 especies ampliamente distribuidas a lo largo de América del Norte, desde el extremo norte de América del Sur hasta el Canadá (Pinto \& Bologna, 1999). En México Pinto (1991) recoge la presencia de 55 especies, pero muchas otras, como E. (M.) fabricii (LeConte, 1853), $E$. (M.) ingrata Fall, 1907, E. (M.) gissleri (Horn, 1878), E. (M.) parkeri Werner, 1944, etc., se encuentran en Texas, Nuevo México o Arizona (EEUU) muy cerca de la frontera, por lo que su presencia en México es muy probable.

Pinto (1991) considera 14 grupos de especies dentro del subgénero (grupos de E. alastor, E. albida, E. diversicornis, E. dohrni, E. fabricii, E. funesta, E. ingrata, E. lauta, E. ochrea, E. prosopidis, E. tenella, E. tenuis LeConte, 1853, E. uniforma y E. virgulata). Casi todos los grupos de especies tienen representantes en México a excepción de los de E. dohrni (Haag-Rutenberg, 1880) distribuido por Colombia y Panamá, E. fabricii ampliamente extendido en el Canadá y los EEUU y el de $E$. ingrata propio del Suroeste de los EEUU. Otros autores (Werner, 1954) han considerado Gnathospasta como un subgénero independiente.

\section{Epicauta (Macrobasis) alastor Skinner, 1904}

Epicauta alastor Skinner, 1904: 217 (*)

TERRA TYPICA: "Florence, Arizona". Holotipo en la colección de la Academy of Natural Sciences of Philadelphia (EEUU) (fide Skinner, 1904), con el no 8084 (fide Werner, 1945).

COMENTARIOS TAXONÓMICOS: Incluida en el grupo de E. alastor (Werner, 1954b; Pinto, 1991). Werner et al. (1966) la consideran parte del subgénero Gnathospasta.

DISTRIBUCIÓN GEOGRÁFICA: Limitada geográficamente al noroeste de México y sur de los EEUU (Pinto, 1991). En México ha sido citada de los estados de Sinaloa y Sonora.

MÉxICo: Sinaloa: Los Mochis (Werner et al., 1966). Sonora: Ciudad Obregón (Werner et al., 1966); Hermosillo (Werner et al., 1966).

\section{Epicauta (Macrobasis) albida (Say, 1824)}

Lytta albida Say, 1824b: $305(*)$

TERRA TYPICA: "Arkansas... near the Rocky Mountains", originalmente (fide Werner, 1945); "La Junta, Bent Co., Colorado", tras designación de neotipo por Werner (1945) en la colección del Museum of Comparative Zoology (Harvard University, Cambridge, EEUU). 
Lytta luteicornis LeConte, 1854c: $84(*)$

TERRA TYPICA: "Laredo to Ringgold Barracks...”. Lectotipo n 4977 , designado por Werner (1945) en la colección del Museum of Comparative Zoology (Harvard University, Cambridge, EEUU).

Macrobasis albida (Say, 1824): Horn, 1873: 89 (*)

Epicauta albida (Say, 1824): Werner, 1945: 511 (*)

COMENTARIOS TAXONÓMICOS: Incluida en el grupo de E. albida (Selander \& Mathieu, 1969; Pinto, 1991). El propio LeConte (1883) sinonimiza L. luteicornis con $L$. albida.

DiSTRIBUCIÓN GEOGRÁFICA: Extendida por el norte de México y sur de los EEUU (Pinto, 1991). Citada de los estados de Coahuila, Nuevo León, San Luis Potosí y Tamaulipas. Champion (1892) cita E. albida en Orizaba por confusión con E. basalis, como él mismo señala al tratar esta especie (Champion, 1892: 402).

MÉxICO: Coahuila: 12 mi. al N de Hermanas (Selander \& Mathieu, 1969). Nuevo León: Apodaca (Selander \& Mathieu, 1969); Monterrey (Selander \& Mathieu, 1969); Pesquería (Selander \& Mathieu, 1969); Sabinas Hidalgo (Selander \& Mathieu, 1969); Villa de Santiago (Selander \& Mathieu, 1969). San Luis Potosí:. Huasteca Potosina (Dugès, 1889e; Champion, 1892). Tamaulipas: Ciudad Victoria (Selander \& Mathieu, 1969); Nuevo Laredo (Selander \& Mathieu, 1969).

Epicauta (Macrobasis) apicalis Dugès, 1889

Epicauta apicalis Dugès, 1889e: 90 (*)

TERRA TYPICA: "Tepic".

Cantharis apicalis (Dugès, 1889d): Champion, 1899: $172(*)$

COMENTARIOS TAXONÓMICOS: Pinto (1991) señala que la descripción original de E. apicalis, sólo parece diferir de la de E. terminata en la coloración y que, por lo tanto, ambas podrían corresponder a una única especie. Borchmann (1917) la incluye en el género Epicauta (no en Macrobasis). Pinto (1991) mantiene a E. apicalis en el grupo de E. funesta sensu Werner (1954b).

DistRIBUCIÓN GEOGRÁFICA: Especie endémica de México (Pinto, 1991), únicamente conocida de Nayarit.

MÉXICO: Nayarit: Tepic (Dugès, 1889e; Champion, 1892; Zaragoza-Caballero, 1999).

\section{Epicauta (Macrobasis) arizonica Werner, 1944}

Epicauta arizonica Werner, 1944: 72 (*)

TERRA TYPICA: "Bavoquivari Mts., Arizona". Holotipo no 26062, en la colección del Museum of Comparative Zoology (Harvard University, Cambridge, EEUU) (fide Werner, 1944).

COMENTARIOS TAXONÓMICOS: Incluida en el grupo de E. diversicornis (Werner, 1949b; Pinto, 1991). Los ejemplares de Meoqui, capturados junto con ejemplares de $E$. polingi, presentan la misma coloración que éstos (Werner et al., 1966). Según Werner et al. (1966) se incluye en el subgénero Gnathospasta.

DisTRIBUCIÓN GEOGRÁFICA: Ampliamente distribuida en el noroeste de México y Arizona (Pinto, 1991). Se ha citado en los estados de Baja California Sur, Chihuahua, Nayarit, Sinaloa y Sonora.
MÉXICo: Baja California Sur: cerca de Santiago (Werner et al., 1966). Chihuahua: $7 \mathrm{mi}$. al O de Chihuahua (Selander, 1954b; Werner et al., 1966); 6 mi. al NE de Meoqui (Werner et al., 1966); Salaices (Werner et al., 1966). Nayarit: San Blas (Werner et al., 1966). Sinaloa: Culiacán (Selander, 1982c). Sonora: Arizpe (Werner, 1944; 1945; 1949a; Werner et al., 1966); $8 \mathrm{~km}$ al O de Carbo (Werner et al., 1966); Ciudad Obregón (Werner et al., 1966); Guaymas (Werner et al., 1966); Hermosillo (Werner et al., 1966).

Epicauta (Macrobasis) atricolor Champion, 1892

Epicauta atricolor Champion, 1892: 419 (*)

TERRA TYPICA: "MeXICO, Matamoros Izucar, Oaxaca..., Jalapa...", originalmente; "Oaxaca, Mexico..." tras designación de lectotipo (Pinto, 1982). Lectotipo en la colección del British Museum of Natural History (Londres). La serie típica consta de cinco ejemplares más (Pinto, 1982), aunque Champion (1892) indica ocho en total.

Cantharis atricolor (Champion, 1892): Champion, 1899: $172(*)$

COMEnTARios taXonómicos: Champion (1892), Borchmann (1917) y Werner (1958) colocaron a esta especie dentro del subgénero Epicauta, pero Pinto (1982) la transfiere a Macrobasis. Incluida en el grupo de E. uniforma (Werner, 1954b; Pinto, 1991). Pinto (1991) señala que E. bipunctata posiblemente sea un sinónimo de esta especie. Champion (1892) indica que un ejemplar de Izúcar de Matamoros (Puebla) podría corresponder a otra especie.

DisTRIBUCIÓN GEOGRÁFICA: Especie endémica de México (Pinto, 1991). Conocida de los estados de Morelos, Oaxaca, Puebla y Veracruz.

MÉxICo: Morelos: (Pinto, 1991). Oaxaca: Oaxaca (Champion, 1892). Puebla: Izúcar de Matamoros (Champion, 1892). Veracruz: Xalapa (Champion, 1892).

\section{Epicauta (Macrobasis) atripilis Champion, 1892}

Epicauta atripilis Champion, 1892: $410(*)$

TERRA TYPICA: "MeXICO, Cordova and Oaxaca...". Sintipos en la colección del British Museum of Natural History (Londres) (fide Pinto 1991).

Cantharis atripilis (Champion, 1892): Champion, 1899: $172(*)$

COMENTARIOS TAXONÓMICOS: Champion (1892) y Borchamnn (1917) sitúan esta especie dentro del subgénero Epicauta, pero Pinto $(1982 ; 1991)$ la considera en Macrobasis y la incluye en el grupo de E. funesta sensu Werner (1954b). Pinto (1991) señala que E. atripilis y E. cinereiventris podrían representar una única especie ya que sólo se diferencian en el color de la pilosidad. Pinto (1991) ha examinado ejemplares de Puebla con la cabeza roja, de posición taxonómica incierta, que podrían pertenecer a esta especie.

DisTRIBUCIÓN GEOGRÁFICA: Endemismo mexicano (Pinto, 1991), distribuido por los estados de Oaxaca y Veracruz.

MÉxICo: Oaxaca: Oaxaca (Champion, 1892). Veracruz: Córdoba (Champion, 1892). 
Epicauta (Macrobasis) atrivittata (LeConte, 1854)

Lytta atrivittata LeConte, 1854d: 224 (*)

TERRA TYPICA: "San Diego trip". Lectotipo, no 4980 (único ejemplar de la serie típica), designado por Werner (1945) en la colección del Museum of Comparative Zoology (Harvard University, Cambridge, EEUU) (fide Selander \& Mathieu, 1969).

Macrobasis atrivittata (LeConte, 1854): Horn, 1873: 90 (*)

Epicauta atrivittata (LeConte, 1854): Werner, 1945: 509 (*)

COMENTARIOS TAXONÓMICOS: Especie incluida en el grupo de E. albida (Selander \& Mathieu, 1969; Pinto, 1991).

DisTRIBUCIÓN GEOGRÁFICA: Extendida por el norte de México y sur de los EEUU (Pinto, 1991). Citada en los estados de Chihuahua, Coahuila y Durango. Selander (1954b) y Werner et al. (1966) indican que también ha sido citada en Tamaulipas, aunque este registro corresponde en realidad al Estado de Durango.

MÉXICo: Chihuahua: $20 \mathrm{mi}$. al SO de Camargo (Vaurie, 1950; Selander \& Mathieu, 1969); 11 mi. al S de Camargo (Selander \& Mathieu, 1969); 3 mi. al N de Camargo (Selander \& Mathieu, 1969); 70 mi. al NNO de Casas Grandes (Selander \& Mathieu, 1969); 10 mi. al S de Delicias (Vaurie, 1950; Selander \& Mathieu, 1969); 10 mi. al N de Jiménez (Selander \& Mathieu, 1969); Villa Ahumada (Selander, 1954b; Selander \& Mathieu, 1969). Coahuila: $12 \mathrm{mi}$ al O de La Rosa (Selander \& Mathieu, 1969). Durango: Tlahualillo de Zaragoza (Werner, 1945; Vaurie, 1950; Werner et al., 1966; Selander \& Mathieu, 1969).

\section{Epicauta (Macrobasis) beckeri Dugès, 1889}

Epicauta bekeri Dugès, 1889e: 113 bis (*)

TERRA TYPICA: "Canelas. Sierra madre de Durango". Macrobasis beckeri (Dugès, 1889): Champion, 1892: 400 (enmienda) (*) Cantharis beckeri (Dugès, 1889): Champion, 1899: 173 (*) Epicauta beckeri Dugès, 1889: Blackwelder, 1945: 482 (*)

COMENTARIOS TAXONÓMICOS: Estrechamente relacionada con E. diversicornis según Champion (1892), e incluida en el grupo de E. diversicornis (Werner, 1949b; Pinto, 1991). La enmienda propuesta por Champion (1892) podría no estar justificada ya que Dugès (1889e) indica que nombra la especie en honor de Ricardo Beker.

DistribuCIÓN GEOGRÁfICA: Endemismo mexicano (Pinto, 1991), encontrado en los estados de Colima, Durango, Jalisco, Morelos y Nayarit.

MÉXICo: Colima: (Pinto, 1991). Durango: Canelas (Dugès, 1889e; Champion, 1892; Werner, 1949a; Vaurie, 1950). Jalisco: Colima Vulcano [Nevado de Colima] (Werner, 1949a). Morelos: (Pinto, 1991). Nayarit: Tepic (Werner, 1949a; Vaurie, 1950).

\section{Epicauta (Macrobasis) bipunctata Werner, 1958}

Epicauta bipunctata Werner, 1958: 7 (*)

TERRA TYPICA: "Chilpancingo, Guerrero, Mexico, 4900'..." Holotipo en la colección del Museum of Comparative Zoology (Harvard University, Cambridge, EEUU) (fide Werner, 1958). Los paratipos están distribuidos en diversas colecciones incluída la del Instituto de Biología (Universidad Nacional Autónoma de México) (fide Werner, 1958)
COMENTARIOS TAXONÓMICOS: Especie conservada en el grupo de E. uniforma sensu Werner (1954b) por Werner (1958), Selander \& Agafitei (1982) y Pinto (1991). Pinto (1991) señala que E. bipunctata posiblemente sea un sinónimo de E. atricolor.

Distribución GEOGRÁfiCA: Especie endémica de México (Pinto, 1991). Distribuida en los estados de Guerrero, Jalisco, Morelos y Sinaloa. Werner (1958) cita ejemplares de Tepic (Nayarit) como pertenecientes con dudas a esta especie.

MÉXICo: Guerrero: Acahuizotla (Werner, 1958); Chilpancingo (Werner, 1958); Mexcala (Werner, 1958). Jalisco: Guadalajara (Werner, 1958); Zapotlanejo (Werner, 1958). Morelos: Hujintlán (Werner, 1958); Tepoztlán (Werner, 1958). Sinaloa: Culiacán (Selander \& Agafitei, 1982).

Epicauta (Macrobasis) borrei (Dugès, 1881)

Lytta fumosa Haag-Rutenberg, 1880: 40 (non Germar, 1824) (*)

TERRA TYPICA: "Mexico". Holotipo posiblemente en la colección Haag-Rutenberg del Zoologische Staatsammlung de Munich.

Cantharis borrei Dugès, 1881: 145 (*)

TERRA TYPICA: "hacienda de Tupátaro, ....al pié del cerro... Fuerte de San Gregorio y Cueva del Padre Torres". Holotipo en paradero desconocido.

Macrobasis borrei (Dugès, 1881): Horn, 1885: 107 (*)

Macrobasis fumosa (Haag-Rutenberg, 1880): Champion, 1892: 395 (*) Cantharis fumea Champion, 1899: 178 (lapsus) (*)

Epicauta fumosa (Haag-Rutenberg, 1880): Beauregard, 1890: 504 (*) Epicauta borrei (Dugès, 1881): Denier, 1935a: $153\left({ }^{*}\right)$

COMENTARIOS TAXONÓMICOS: Incluida provisionalmente en el grupo de E. uniforma sensu Werner (1954b) por Pinto (1991).

DisTRIBUCIÓN GEOGRÁfICA: Endemismo mexicano (Pinto, 1991), ampliamente distribuida en los estados de Aguascalientes, Durango, Guanajuato, Jalisco, México, Michoacán y Zacatecas.

MÉXICo: Aguascalientes: (Pinto, 1991). Durango: (Pinto, 1991). Guanajuato: Fuerte de San Gregorio (Dugès, 1881, 1889e); Guanajuato (Champion, 1892); Tupátaro (Dugès, 1881, 1889e; Champion, 1892; Zaragoza-Caballero, 1999). Jalisco: (Pinto, 1991). México: (Pinto, 1991). Michoacán: (Pinto, 1991). Zacatecas: (Pinto, 1991).

Epicauta (Macrobasis) candezi (Haag-Rutenberg, 1880) Lytta candezi Haag-Rutenberg, 1880: 43 (*)

TERRA TYPICA: "Guatimala (sic)". Posiblemente en la colección Haag-Rutenberg del Zoologische Staatsammlung (Munich).

Macrobasis candezi (Haag-Rutenberg, 1880): Beauregard, 1890: 500 (*) Cantharis candezei (Haag-Rutenberg, 1880): Champion, 1899: 176 (y error tipográfico) (*)

Epicauta candezi (Haag-Rutenberg, 1880): Werner, 1949a: 76 (*)

Comentarios taxonómicos: Champion (1892), seguido por Borchmann (1917: 87, bajo el nombre de Macrobasis candezei), incluyó a $E$. candezi en la sinonimía de E. diversicornis; Denier (1935a), seguido por Blackwelder (1945), la trató como variedad de $E$. diversicornis, situación que se mantuvo hasta que finalmente Werner (1949a) la restituyó como especie válida. 
Incluida en el grupo de E. diversicornis (Werner, 1949b; Pinto, 1991).

DisTRIBUCIÓN GEOGRÁFICA: Especie conocida de El Salvador, Guatemala y México (Werner, 1949a; Pinto, 1991). Blackwelder (1945) señala también su presencia en Costa Rica. Citada en los estados de Chiapas y Yucatán.

MÉXICO: Chiapas: (Werner, 1949a; Pinto, 1991). Yucatán: (Pinto, 1991).

Epicauta (Macrobasis) cinereiventris Champion, 1892

Epicauta cinereiventris Champion, 1892: 411 (*)

TERRA TYPICA: "MeXICO, Acaguizotla in Guerrero; Guatemala, San Gerónimo, Chimaltenango". Diez sintipos posiblemente en la colección del British Museum of Natural History (Londres)

Cantharis cinereiventris (Champion, 1892): Champion, 1899: 174 (*)

COMENTARIOS TAXONÓMICOS: Pinto (1991) señala que E. atripilis y E. cinereiventris podrían corresponder a una única especie. Champion (1892), seguido por Borchamnn (1917), sitúa a esta especie dentro del subgénero Epicauta, pero Pinto $(1982 ; 1991)$ la considera dentro de Macrobasis y la incluye en el grupo de $E$. funesta sensu Werner (1954b).

DisTRIBUCIÓN GEOGRÁFICA: Especie presente en México, Guatemala y Nicaragua (Champion, 1892; Maes \& Huether, 2007), distribuida por los estados de Chiapas, Guerrero y Oaxaca .

MÉxICO: Chiapas: (Pinto, 1991). Guerrero: Acaguizotla (Champion, 1892). Oaxaca: Tehuantepec (Champion, 1892; Pinto, 1991).

\section{Epicauta (Macrobasis) croceicincta (Dugès, 1881)}

Cantharis croceicincta Dugès, 1881: 143 (*)

TERRA TYPICA: "Guanajuato y ... hacienda de Tupátaro". Holotipo en paradero desconocido.

Epicauta croceicincta (Dugès, 1881): Dugès, 1886a: 582 (*)

COMENTARIOS TAXONÓMICOS: Epicauta pacifica parece ser una variante de color de E. croceicincta (Pinto, 1991). Epicauta croceicincta ha sido incluida en Epicauta por Beauregard (1890) y Borchmann (1917) y en Macrobasis por Pinto (1991), autor que además la incluye en el grupo de E. funesta sensu Werner (1954b). En la obra de Dugès (1889e: 79) se incluyó el error tipográfico Epicauta groceicincta, mientras que en ZaragozaCaballero (1999: 22) se deslizó el nombre Epicauta croceisincta.

DisTRIBUCIÓN GEOGRÁFICA: Especie endémica de México (Pinto, 1991), localizada en los estados de Guanajuato y Jalisco.

MÉxICo: Guanajuato: Guanajuato (Dugès, 1881; Champion, 1892; Zaragoza-Caballero, 1999); Tupátaro (Dugès, 1881, 1889e; Champion, 1892; Zaragoza-Caballero, 1999). Jalisco: Lake Chapala [Laguna de Chapala] (Pinto, 1991).
Epicauta (Macrobasis) disparilis (Champion, 1892)

Macrobasis disparilis Champion, 1892: 398 (*)

Terra typica: "Mexico, Puebla, Omilteme in Guerrero 8000 feet".

Siete sintipos, posiblemente en la colección del British Museum of

Natural History (Londres).

Cantharis disparilis (Champion, 1892): Champion, 1899: 176 (*) Epicauta disparilis (Champion, 1892): Blackwelder, 1945: 483 (*)

COMENTARIOS TAXONÓMICOS: Especie incluida en el grupo de E. prosopidis (Werner, 1973; Pinto, 1991).

Distribución GeOgRÁfiCA: Endémica de México (Pinto, 1991). Sólo conocida de los estados de Guerrero y Puebla.

MÉxICo: Guerrero: Omilteme (Champion, 1892). Puebla: Puebla (Champion, 1892).

\section{Epicauta (Macrobasis) distorta (Champion, 1892)}

Macrobasis distorta Champion, 1892: 396 (*)

TERRA TYPICA: "Mexico, Iguala in Guerrero, Jalapa, Orizaba; Nicaragua, Chontales". Cinco sintipos, posiblemente en la colección del British Museum of Natural History (Londres).

Cantharis distorta (Champion, 1892): Champion, 1899: 176 (*)

Epicauta distorta (Champion, 1892): Blackwelder, 1945: 483 (*)

COMENTARIOS TAXONÓMICOS: Incluida en el grupo de E. prosopidis (Werner, 1973; Pinto, 1991).

DisTRIBUCIÓN GEOGRÁFICA: Ampliamente distribuida desde el sur de México hasta Costa Rica, con citas en Honduras y Nicaragua (Pinto, 1991; Maes \& Huether, 2007). Pinto (1991) únicamente recoge la cita mexicana de Guerrero, pero Champion (1892) también cita la especie en Veracruz.

MÉxICo: Guerrero: Iguala (Champion, 1892). Veracruz: Xalapa (Champion, 1892); Orizaba (Champion, 1892);

Epicauta (Macrobasis) diversicornis (Haag-Rutenberg, 1880)

Lytta diversicornis Haag-Rutenberg, 1880: 42 (*)

TERRA TYPICA: “Mexico (Buliacan) (sic)...”. Posiblemente en la colección Haag-Rutenberg del Zoologische Staatsammlung de Munich.

Lytta diversicornis var. pallida Haag-Rutenberg, 1880: 42 (*)

TERRA TYPICA: "Mexico...". Holotipo en el Helsingforser MuseumsSammlung (fide Haag-Rutenberg, 1880).

Macrobasis flavens Dugès, 1889e: 58 (*)

TERRA TYPICA: "Acapulco". Vázquez \& Zaragoza (1979) indican como tipo el ejemplar n ${ }^{0} 2887$ (IBUNAM 024) de la colección del Instituto de Biología de la Universidad Nacional Autónoma de México.

Macrobasis diversicornis (Haag-Rutenberg, 1880): Beauregard, 1890: $500(*)$

Cantharis diversicornis (Haag-Rutenberg, 1880): Champion, 1899: $176(*)$

Epicauta diversicornis (Haag-Rutenberg, 1880): Blackwelder, 1945: $483(*)$

COMENTARIOS TAXONÓMICOS: Champion (1892) y Borchmann (1917) incluyen a $E$. candezi en la sinonimia de $E$. diversicornis, criterio no compartido por Pinto (1991). Incluida en el grupo de E. diversicornis (Werner, 1949b; Pinto, 1991).

DistribuCión GEOGRÁFICA: Especie endémica de México (Pinto, 1991). Citada en los estados de Distrito 
Federal, Guerrero, Hidalgo, Jalisco, México, Michoacán, Morelos, Nayarit, Sinaloa, Sonora y Veracruz. Vaurie (1950) indica que las citas de Champion (1892) de E. diversicornis de Chihuahua y Durango, corresponden a E. polingi. Pinto (1991) no incluye en su listado las citas del Distrito Federal y de Nayarit recogidas por Werner (1949a).

MÉxICo: Distrito Federal: Ciudad de México (Werner, 1949a). Guerrero: Acapulco (Dugès, 1889e sub M. flavens; Champion, 1892; Werner, 1949a; Vázquez \& Zaragoza, 1979 sub M. flavens; Zaragoza-Caballero, 1999 sub M. flavens); Chilpancingo (Champion, 1892). Hidalgo: Pachuca (Werner, 1949a). Jalisco: Guadalajara (Werner, 1949a); Sayula (Champion, 1892). México: (Pinto, 1991). Michoacán: Apatzingan (Werner, 1949a). Morelos: Cuernavaca (Werner, 1949a). Nayarit: Tepic (Werner, 1949a). Oaxaca: Tehuantepec (Champion, 1892). Sinaloa: Buliacan [Culiacán] (HaagRutenberg, 1880; Champion, 1892); Mazatlán (Werner, 1949a); Venodio (posiblemente Venadillo, ver Selander, 1960a) (Werner, 1949a). Sonora: (Pinto, 1991). Veracruz: Córdoba (Werner, 1949a); Xalapa (Champion, 1892); Tuxtla (Champion, 1892).

\section{Epicauta (Macrobasis) evanescens Champion, 1892} Epicauta evanescens Champion, 1892: 412 (*)

Terra typica: "Guatemala, Champerico". Tres sintipos, posiblemente en la colección del British Museum of Natural History (Londres).

Cantharis evanescens (Champion, 1892): Champion, 1899: 177 (*)

COMENTARIOS TAXONÓMICOS: Incluida en Epicauta por Champion (1892) y Borchmann (1917), Pinto (1991) la incluye en el subgénero Macrobasis, dentro del grupo de E. funesta sensu Werner (1954b).

DistribuCiÓN GEOGRÁFICA: Localizada en México y Guatemala (Pinto, 1991). Los únicos registros mexicanos corresponden al estado de Chiapas.

MÉXICO: Chiapas: (Pinto, 1991).

Epicauta (Macrobasis) excors (Fall, 1909)

Macrobasis excors Fall, 1909: 166 (*)

TERRA TYPICA: "El Taste, Lower California". Holotipo no 24296, en la colección del Museum of Comparative Zoology (Harvard University, Cambridge, EEUU) (fide Werner, 1945)

Epicauta excors (Fall, 1909): Werner, 1945: 503 (*)

Comentarios taxonómicos: Pinto (1991) indica que podría tratarse de una forma de coloración obscura de $E$. languida. Incluida en el grupo de E. tenuis (Selander \& Mathieu, 1969; Pinto, 1991).

Distribución GEOGRÁFICA: Especie endémica de la Península de Baja California (Pinto, 1991). Sólo conocida de Baja California Sur.

MÉXICO: Baja California Sur: El Taste (Fall, 1909; Werner, 1945); Santa Rosa (Werner, 1945).

Epicauta (Macrobasis) forticornis (Haag-Rutenberg, 1880)

Lytta forticornis Haag-Rutenberg, 1880: 41 (*)
TERRA TYPICA: "Mexico". Tipo en el Helsingforser MuseumsSammlung (fide Haag-Rutenberg, 1880).

Macrobasis forticornis (Haag-Rutenberg, 1880): Beauregard, 1890: $500(*)$

Cantharis forticornis (Haag-Rutenberg, 1880): Champion, 1899: 178 (*)

Epicauta forticornis (Haag-Rutenberg, 1880): Blackwelder, 1945: 483 (*)

COMENTARIOS TAXONÓMICOS: Incluida en el grupo de E. diversicornis (Werner, 1949b; Pinto, 1991).

DisTRIBUCIÓN GEOGRÁfICA: Endemismo mexicano (Pinto, 1991), citado en los estados de Guerrero, Morelos, Nayarit y Yucatán.

MÉXICo: Guerrero: (Pinto, 1991). Morelos: Cuernavaca (Werner, 1949a). Nayarit: Tepic (Werner, 1949a). Yucatán: Chichén Itzá (Werner, 1949a).

Epicauta (Macrobasis) funesta (Chevrolat, 1834)

Lytta funesta Chevrolat, 1834a: fasc. $3, \mathrm{n}^{\circ} 58$ (sin paginar) (*) TERRA TYPICA: "Orixaba".

Cantharis funesta (Chevrolat, 1834): Dugès, 1869b: 67 (*)

Epicauta funesta (Chevrolat, 1834): Dugès, 1886a: $582(*)$

COMENTARIOS TAXONÓMICOS: Incluida en el subgénero nominal por Dugès (1889e) y Beauregard (1890), Pinto (1991) la incluye en Macrobasis, dentro del grupo de E. funesta sensu Werner (1954b).

DistribuCiÓN GEOGRÁFICA: Especie endémica de México (Pinto, 1991), localizada en los estados de Puebla y Veracruz.

MÉxICo: Puebla: (Champion, 1892; Pinto, 1991). Veracruz: Almolonga (Dugès, 1889e; Champion, 1892); Córdoba (Champion, 1892); El Camarón [en Veracruz según Selander \& Vaurie, 1962] (Champion, 1892); Orizaba (Chevrolat, 1834a; Dugès, 1869b; Champion, 1892); Veracruz (Dugès, 1889e; Champion, 1892).

Epicauta (Macrobasis) hirsutipubescens (Maydell, 1934) Macrobasis hirsutipubescens Maydell, 1934: 334 (*)

TERRA TYPICA: “...Texas...”. Holotipo no 8181, en la colección Horn de la American Entomological Society (EEUU) (fide Maydell, 1934).

Epicauta hirsutipubescens (Maydell, 1934): Werner, 1949b: 110 (*)

COMENTARIOS TAXONÓMICOS: Werner (1945) trató a esta especie como sinónimo de E. virgulata, pero posteriormente restablece su estatus específico (Werner, 1949b). Incluida en el grupo de E. virgulata (Werner, 1954b; Pinto, 1991) y en el subgénero Gnathospasta por Werner et al. (1966).

DisTRIBUCIÓN GEOGRÁFICA: Extendida por el norte de México y sur de los EEUU (Pinto, 1991). En México ha sido citada de los estados de Chihuahua, Coahuila, Durango y Sonora.

MÉXICo: Chihuahua: 20 mi. al SO de Camargo (Vaurie, 1950); 25 mi. al SO de Camargo (Vaurie, 1950); $42 \mathrm{mi}$. al SO de Camargo (Vaurie, 1950); Delicias (Vaurie, 1950); 20 mi. al $\mathrm{S}$ de Ciudad Juárez (Selander, 1954b); Samalayuca (Vaurie, 1950). Coahuila: Cabos (Vaurie, 1950); Guadalupe (Vaurie, 1950); La Gloria, al S de Monclova (Vaurie, 1950); Paila (Vaurie, 1950); 5 mi. al N de Saltillo (Vaurie, 1950); 25 mi. al 
SE de San Pedro de Colonias (Vaurie, 1950). Durango: Pedricena (Vaurie, 1950; Werner et al., 1966). Sonora: Ciudad Obregón (Werner et al., 1966); Hermosillo (Werner et al., 1966); Naco (Vaurie, 1950).

Epicauta (Macrobasis) humeralis (Dugès, 1889)

Macrobasis humeralis Dugès, 1889e: 58 (*)

TERRA TYPICA: "Tepic". Holotipo posiblemente perdido (Pinto, 1991).

Cantharis humeralis (Dugès, 1889): Champion, 1899: 180 (*)

Epicauta humeralis (Dugès, 1889): Blackwelder, 1945: 483 (*)

COMENTARIOS TAXONÓMICOS: Incluida en el grupo de

E. diversicornis (Werner, 1949b; Pinto, 1991).

DiSTRIBUCIÓN GEOGRÁFICA: Especie sólo conocida de México (Pinto, 1991). Presente en los estados de Morelos, Jalisco y Nayarit.

MÉxICo: Morelos: Cañon del Lobo [Cañón de Lobos] (Pinto, 1991); Cuernavaca (Werner, 1949a). Jalisco: Guadalajara (Werner, 1949a). Nayarit: Tepic (Dugès, 1889e; Champion, 1892; Werner, 1949a; Zaragoza-Caballero, 1999).

Epicauta (Macrobasis) immaculata (Say, 1824)

Lytta immaculata Say, 1824b: 304 (*)

TERRA TYPICA: "Arkansa", originalmente; "Cambridge, Nebraska" tras designación de neotipo. Neotipo designado por Werner (1945) en la colección del Museum of Comparative Zoology (Harvard University, Cambridge, EEUU).

Lytta articularis Say, 1824b: 304 (*)

TERRA TYPICA: "Arkansa", “... near the Rocky Mountains”. Tipo perdido (fide Selander \& Mathieu, 1969).

Lytta fulvescens LeConte, 1854a: 447 (*)

TERRA TYPICA: "Texas". Lectotipo no 4989 designado por Werner (1945) en la colección del Museum of Comparative Zoology (Harvard University, Cambridge, EEUU).

Macrobasis immaculata (Say, 1824): Horn, 1873: 93 (*)

Cantharis basalis Dugès, 1881: 144 (*)

TERRA TYPICA: "Estado de Veracruz". Vázquez \& Zaragoza (1979) indican como tipo el ejemplar $\mathrm{n}^{\circ} 1739$ (IBUNAM 021) de la colección del Instituto de Biología de la Universidad Nacional Autónoma de México, aunque Pinto (1991) indica que ese ejemplar no puede ser el tipo ya que corresponde a $E$. (Epicauta) major.

Epicauta basalis (Dugès, 1881): Dugès, 1886a: $582\left({ }^{*}\right)$

Macrobasis basalis (Dugès, 1881): Champion, 1892: 402 (*)

Epicauta immaculata (Say, 1824): Werner, 1945: 489

COMENTARIOS TAXONÓMICOS: Say (1824b) describió con el nombre de L. articularis el macho de la especie. Pinto (1991) señala que el ejemplar registrado como tipo de $C$. basalis en la colección del Instituto de Biología de la Universidad Nacional Autónoma de México (Vázquez \& Zaragoza, 1979) no se corresponde con la descripción de $C$. basalis de Dugès y por lo tanto no puede ser el tipo. Los rasgos indicados tanto en la descripción de Dugès (1881) como en la posterior de Champion (1892) sitúan a $C$. basalis en el subgénero Macrobasis, mientras que el ejemplar en cuestión corresponde a la especie $E$. (Epicauta) major. El tipo de C. basalis, por lo tanto, está perdido, aunque posiblemente perteneciese al mismo lote que los conservados en el British Museum of Natural History de Londres, que responden a las descripciones de Dugès (1881) y Champion (1892) y fueron enviados, como el ejemplar de Dugès, por A. Salle. Tras el examen de estos ejemplares, Pinto (1991) concluye que $C$. basalis es un sinónimo de E. immaculata. Dugès (1881) en la descripción original de $C$. basalis indica el nombre no disponible Cantharis basalis Chevrolat.

Incluida en el grupo de E. albida (Selander \& Mathieu, 1969; Pinto, 1991).

DISTRIBUCIÓN GEOGRÁFICA: Extendida por el norte y este de México y por una amplia región del centro y este de los EEUU (Pinto, 1991). En México se ha citado en los estados de Coahuila, Distrito Federal, Tamaulipas y Veracruz. Aunque Selander \& Mathieu (1969) dudaban de la procedencia real del material de Tampico, Pinto (1991) lo considera aceptable.

MÉxICo: Coahuila: $12 \mathrm{mi}$. al N de Hermanas (Selander \& Mathieu, 1969). Distrito Federal: México (Dugès, 1889e sub E. basalis). Tamaulipas: Tampico (Selander \& Mathieu, 1969). Veracruz: Orizaba (Champion, 1892; Pinto, 1991).

Epicauta (Macrobasis) isthmica Werner, 1949

Epicauta isthmica Werner, 1949a: 72 (*)

Terra tyPICA: "Cabima, Panama". Holotipo en la colección del USNM (fide Werner, 1949a). La serie típica incluye numerosos paratipos.

COMENTARIOS TAXONÓMICOS: Incluida en el grupo de E. diversicornis (Werner, 1949b; Pinto, 1991).

DistRIBUCIÓN GEOGRÁFICA: Especie con una distribución muy dispersa, citada en México, Belice, Costa Rica, Honduras, Nicaragua y Panamá (Pinto, 1991; Maes \& Huether, 2007). En México se ha localizado en los estados de Querétaro y Veracruz.

MÉxICo: Querétaro: (Pinto, 1991). Veracruz: Atoyac (Werner, 1949a); La Gloria, Cardel (Werner, 1949a).

\section{Epicauta (Macrobasis) labialis (Dugès, 1881)}

Cantharis labialis Dugès, 1881: 145 (*)

TERRA TYPICA: "Pénjamo". Holotipo pérdido (fide Werner, 1958). Macrobasis labialis (Dugès, 1881): Dugès, 1886: 582 (*) Gnathospasta labialis (Dugès, 1881): Champion, 1892: 403 (*) Epicauta labialis (Dugès, 1881): Selander, 1954b: 86 (*)

COMENTARIOS TAXONÓMICOS: Champion (1892) y Pinto (1991) señalan que E. labialis es posiblemente un sinónimo de E. mimetica Horn, 1875, una especie de momento sólo conocida en Texas (EEUU).

DistribuCión GEOGRÁfICA: Endemismo mexicano (Pinto, 1991), citado en los estados de Guanajuato y Jalisco.

MÉXICO: Guanajuato: Pénjamo (Dugès, 1881, 1889e; Champion, 1892; Werner, 1958; Zaragoza-Caballero, 1999). Jalisco: 16 mi. al SE de Lagos de Moreno (Selander, 1954b).

Epicauta (Macrobasis) languida (Horn, 1895)

Macrobasis languida Horn, 1895: 252 (*)

TERRA TYPICA: "San José del Cabo". Tipos posiblemente en la colección del Museum of Comparative Zoology de Harvard (EEUU). Werner (1945) indica que no examinó ningún ejemplar de esta especie en su revisión. 
Cantharis languida (Horn, 1895): Champion, 1899: 181 (*)

Epicauta languida (Horn, 1895): Werner, 1945: 501 (*)

COMENTARIOS TAXONÓMICOS: Incluida en el grupo de

E. tenuis (Selander \& Mathieu, 1969; Pinto, 1991).

Distribución GEOGRÁFICA: Especie endémica de la Península de Baja California (Pinto, 1991). Sólo conocida de Baja California Sur.

MÉxICo: Baja California Sur: San José del Cabo (Horn, 1895; Werner, 1945).

\section{Epicauta (Macrobasis) lauta (Horn, 1885)}

Epicauta (Macrobasis) lauta lauta (Horn, 1885)

Macrobasis lauta Horn, 1885: 108 (*)

TERRA TYPICA: “Arizona”. Lectotipo n 8079 , designado por Werner (1945), inicialmente en la colección de la Academy of Natural Sciences of Philadelphia, posteriormente transferido al Museum of Comparative Zoology de Harvard (EEUU)

Epicauta compressicollis Champion, 1892: 427 (*)

TERRA TYPICA: "MeXICO, Villa Lerdo in Durango". Posiblemente en la colección del British Museum of Natural History (Londres). Cantharis compressicollis (Champion, 1892): Champion, 1899: 174 (*)

Cantharis lauta (Horn, 1885): Champion, 1899: 181 (*)

Epicauta macroflexi Dillon, 1952: 414 (*)

TERRA TYPICA: "Big Bend Region, Texas".

Epicauta lauta (Horn, 1885): Werner, 1945: 492 (*)

\section{Epicauta (Macrobasis) lauta rossi Werner, 1949}

Epicauta lauta rossi Werner, 1949b: 108 (*)

TERRA TYPICA: "15 mi. S. of San Domingo, Lower California". Holotipo $\mathrm{n}^{\mathrm{0}}$ 6127, en la colección de la California Academy of Sciences (San Francisco, EEUU) (fide Werner, 1949b).

COMENTARIOS TAXONÓMICOS: Borchmann (1917) trata a E. compressicollis como una especie válida del subgénero Epicauta. Pinto (1991) mantiene a E. lauta tentativamente en el grupo de E. lauta creado por Selander (1982c), a diferencia de Werner (1954b) que la incluyó en el grupo de E. diversicornis. Werner et al. (1966) la consideran parte del subgénero Gnathospasta.

DISTRIBUCIÓN GEOGRÁFICA: Especie distribuida por el norte de México y sur de los EEUU (Pinto, 1991). En México se ha citado en los estados de Baja California Sur, Chihuahua, Coahuila, Durango y Sonora. Las dos subespecies se encuentran en México.

Epicauta lauta lauta: MÉxICo: Chihuahua: Delicias (Vaurie, 1950); $10 \mathrm{mi}$. al S de Delicias (Vaurie, 1950); $25 \mathrm{mi}$. al SO de Camargo (Vaurie, 1950); 42 mi. al SO de Camargo (Vaurie, 1950); Río San Pedro, 49 mi. al SE de Chihuahua (Selander, 1954b); Villa Ahumada (Selander, 1954b). Coahuila: San Pedro de Colonias (Vaurie, 1950; Werner et al., 1966). Durango: Ciudad Lerdo (Champion, 1892 sub E. compressicollis; Vaurie, 1950); Pedricena (Vaurie, 1950). Sonora: Sonoyta (Selander, 1958).

Epicauta lauta rossi: MÉXICo: Baja California Sur: 15 mi. al S de Santo Domingo (Werner, 1949b).

Epicauta (Macrobasis) leoni Dugès, 1889

Epicauta leoni Dugès, 1889e: 74 (*)
TERRA TYPICA: "Morelia". Vázquez \& Zaragoza (1979) indican como tipo el ejemplar $n^{\circ} 2206$ (IBUNAM 026) de la colección del Instituto de Biología de la Universidad Nacional Autónoma de México.

Cantharis leoni (Dugès, 1889): Champion, 1899: 181 (*)

COMENTARIOS TAXONÓMICOS: Incluida en el subgénero nominal por Champion (1892) y Borchmann (1917), pero tratada en el subgénero Macrobasis, dentro del grupo de E. uniforma de Werner (1954b, 1958), por Selander \& Agafitei (1982) y Pinto (1991).

DistribuCión GEOGRÁFICA: Especie endémica de México (Pinto, 1991). Ampliamente distribuida por los estados de México, Michoacán, Nuevo León, San Luis Potosí y Tamaulipas.

MÉXICo: México: Chapingo (Werner, 1958; Selander \& Agafitei, 1982); Tenango del Valle (Champion, 1892). Michoacán: Morelia (Dugès, 1889e; Champion, 1892; Werner, 1958; Vázquez \& Zaragoza, 1979; ZaragozaCaballero, 1999). Nuevo León: (Pinto, 1991). San Luis Potosí: (Pinto, 1991). Tamaulipas: (Pinto, 1991).

\section{Epicauta (Macrobasis) liebecki Werner, 1944}

Epicauta liebecki Werner, 1944: 72 (*)

TERrA TYPICA: "Tucson, Arizona". Holotipo no 26068, en la colección del Museum of Comparative Zoology (Harvard University, Cambridge, EEUU) (fide Werner, 1944).

COMENTARIOS TAXONÓMICOS: Incluida en el grupo de E. diversicornis (Werner, 1949b; Pinto, 1991). Según Werner et al. (1966) se incluye en el subgénero Gnathospasta.

Distribución GEOGRÁfiCA: Noroeste de México y Arizona (Werner, 1949a; Pinto, 1991). Se ha citado en el estado de Sonora, aunque Pinto (1991) no recoge la presencia de esta especie en México.

MÉXICo: Sonora: Imuris (Werner, 1944, 1945, 1949a; Werner et al., 1966).

\section{Epicauta (Macrobasis) linearis (LeConte, 1858)}

Lytta linearis LeConte, 1858a: 23 (*)

TERRA TYPICA: "Llano Estacado...". Holotipo, nº 4986, en la colección del Museum of Comparative Zoology (Harvard University, Cambridge, EEUU) (fide Werner, 1945).

Macrobasis linearis (LeConte, 1858): Horn, 1873: $94(*)$

Epicauta linearis (LeConte, 1858): Werner, 1945: 513 (*)

COMENTARIOS TAXONÓMICOS: Incluida en el grupo de E. virgulata (Werner, 1954b; Pinto, 1991) y en el subgénero Gnathospasta por Werner et al. (1966).

DistrIBUCIÓN GEOGRÁFICA: Especie distribuida en el norte de México y sur de los EEUU (Horn, 1878; Pinto, 1991). En México se ha citado en los estados de Chihuahua y Durango.

MÉxICo: Chihuahua: 6 mi. al NE de Meoqui (Werner et al., 1966). Durango: $35 \mathrm{mi}$. al S de El Entronque (Selander, 1954b; Werner et al., 1966); Pedricena (Vaurie, 1950; Werner et al., 1966). 
Epicauta (Macrobasis) longicollis (LeConte, 1853)

Lytta longicollis LeConte, 1853: $343(*)$

TERRA TYPICA: "Missouri Territory and Santa Fe", originalmente

"New Mexico, Santa Fe", tras designación de lectotipo. Lectotipo n ${ }^{\circ}$

4978, designado por Werner (1945) en la colección del Museum of

Comparative Zoology (Harvard University, Cambridge, EEUU)

Macrobasis longicollis (LeConte, 1853): Horn, 1873: 90 (*)

Epicauta longicollis (LeConte, 1853): Werner, 1945: 508 (*)

Comentarios taXonómicos: Borchmann (1917) siguiendo a Horn (1873), Beauregard (1890) y Champion (1892), incluye a $L$. sublineata en la sinonimia de E. longicollis, criterio no compartido por Werner (1945) ni por Pinto (1991). Incluida en el grupo de $E$. albida (Selander \& Mathieu, 1969; Pinto, 1991).

DisTRIBUCIÓN GEOGRÁFICA: Especie circunscrita al norte de México y sur de los EEUU (Pinto, 1991). En México se ha citado en los estados de Chihuahua y Coahuila. La cita del Norte de Sonora señalada por Champion (1892) y recogida por Vaurie (1950) no ha sido incluida, ya que Selander \& Mathieu (1969) indican que es muy probable que corresponda a un ejemplar de Arizona etiquetado de Sonora por error.

MÉxICO: Chihuahua: Ojo de Laguna (Vaurie, 1950; Selander \& Mathieu, 1969); $182 \mathrm{~km}$ al N de Chihuahua (Vaurie, 1950; Selander \& Mathieu, 1969); $10 \mathrm{mi}$. al S de Delicias (Vaurie, 1950; Selander \& Mathieu, 1969). Coahuila: Serranía de los Burros (Selander \& Mathieu, 1969).

Epicauta (Macrobasis) melanochroa Wellman, 1910 Cantharis nigra Dugès, 1870: 161 (non Cantharis nigra Thunberg, 1787) $(*)$

TERRA TYPICA: “...Michoacan, hacienda de la Noria...”. Vázquez \& Zaragoza (1979) indican como tipo el ejemplar $n^{\circ} 809$ (IBUNAM 009) de la colección del Instituto de Biología de la Universidad Nacional Autónoma de México.

Epicauta nigra (Dugès, 1870): Dugès, 1886a: 582 (*)

Epicauta melanochroa Wellman, 1910c: 24 (nombre de substitución para Cantharis nigra Dugès, 1870) (*)

COMENTARIOS TAXONÓMICOS: La homonimia primaria del nombre propuesto por Dugès, (1870) con Cantharis nigra Thunberg, 1787, precede a la homonimia secundaria, indicada por Wellman (1910c) y seguida por Pinto (1991) con Meloe nigra Woodhouse, 1800, un sinónimo de Cantharis pensylvanica De Geer, 1775 (Borchmann, 1917; Pinto, 1991). Champion (1892) con dudas, y Borchmann (1917), incluyen además, a E. funebris (Horn, 1873) en la sinonimia de E. nigra, aunque ni Denier (1935b) ni Pinto (1991) comparten su criterio.

Beauregard (1890), Champion (1892) y Borchmann (1917) incluyen a $E$. nigra en el subgénero nominal, mientras que Pinto (1991) la incluye dentro de Macrobasis, en el grupo de E. uniforma sensu Werner (1954b).

DistribuCión GEOGRÁfICA: Especie endémica de México (Pinto, 1991). Distribuida por los estados del Distrito Federal, Guanajuato, Jalisco, México, Michoacán, Morelos, Nayarit y Querétaro. Champion (1892) indica que las citas de Xalapa (Dugès, 1889e; Zaragoza-Caballero, 1999) necesitan confirmación.

MÉxICo: Distrito Federal: Guadalupe (Werner, 1958); Tlalpan (Werner, 1958). Guanajuato: Moroleón (Zaragoza-Caballero, 1999 sub E. nigra); León (Zaragoza-Caballero, 1999 sub E. nigra); Pénjamo (Zaragoza-Caballero, 1999 sub E. nigra); Tupátaro (Zaragoza-Caballero, 1999 sub E. nigra). Jalisco: Ahualulco (Zaragoza-Caballero, 1999 sub E. nigra); Atenquique (Champion, 1892 sub E. nigra); 11 mi. al N de Chapala (Werner, 1958); Guadalajara (Dugès, 1889e sub E. nigra; Champion, 1892 sub E. nigra; Werner, 1958); Lagos de Moreno (Werner, 1958); El Molino (Werner, 1958); $10 \mathrm{~km}$ al $\mathrm{N}$ de Tepatitlán (Werner, 1958); Tepatitlán (Werner, 1958); $9 \mathrm{~km}$ al N de Mazamitla (Werner, 1958); $40 \mathrm{mi}$. al NE de Tamazula (Werner, 1958); Zapopan (Werner, 1958). México: Atlacomulco (Werner, 1958); Teotihuacán (Werner, 1958). Michoacán: Chavinda (Werner, 1958); $11 \mathrm{~km}$ al E de Chupicuaro (Werner, 1958); Hacienda "La Noria", a 5 leguas de la Piedad (Dugès, 1870; Champion, 1892 sub E. nigra; Vázquez \& Zaragoza, 1979 sub C. nigra); Jacona (Werner, 1958); Morelia (Dugès, 1889e sub E. nigra; Champion, $1892 \mathrm{sub}$ E. nigra); $17 \mathrm{~km}$ al E de Quiroga (Werner, 1958). Morelos: Tepoztlán (Werner, 1958). Nayarit: Tepic (Werner, 1958). Querétaro: cerca de la Hacienda Balvanera (Werner, 1958). Veracruz: Almolonga (Zaragoza-Caballero, 1999 sub E. nigra); Xalapa (Dugès, 1889e sub E. nigra; Zaragoza-Caballero, 1999).

Epicauta (Macrobasis) nigritibialis Werner, 1958

Epicauta nigritibialis Werner, 1958: 5 (*)

TERRA TYPICA: "Saltillo (Coahuila?)". Holotipo en la colección del Museum of Comparative Zoology (Harvard University, Cambridge, EEUU) (fide Werner, 1958).

COMENTARIOS TAXONÓMICOS: Especie conservada en el grupo de E. uniforma sensu Werner (1954b) por Werner (1958) y Pinto (1991).

DISTRIBUCIÓN GEOGRÁFICA: Endemismo mexicano (Pinto, 1991), sólo conocido de Coahuila.

MÉxICo: Coahuila: Saltillo (Werner, 1958).

Epicauta (Macrobasis) niveolineata (Haag-Rutenberg, 1880)

Lytta niveolineata Haag-Rutenberg, 1880: 46 (*)

TerRa tYPICA: "Mexico". Tipo en el Helsingforser MuseumsSammlung (fide Haag-Rutenberg, 1880).

Epicauta niveolineata (Haag-Rutenberg, 1880): Beauregard, 1890: 508 (*) Cantharis niveolineata (Haag-Rutenberg, 1880): Champion, 1899: 184 (*)

COMENTARIOS TAXONÓMICOS: Beauregard (1890), Champion (1892) y Borchmann (1917) la incluyen en el subgénero nominal; Pinto (1991) la trata dentro de Macrobasis, incluida en el grupo de E. uniforma de Werner (1954b) y conservada allí por Selander \& Agafitei (1982). Haag-Rutenberg (1880) describió posteriormente otra especie del Himalaya con el mismo nombre (ahora E. atkinsoni Beauregard, 1889). Champion $(1892,1899)$ incluye en la sinonimia el nombre no disponible E. tristis Sturm, 1843: 175.

DistribuCión GEOGRÁFICA: Especie endémica de México (Pinto, 1991). Extendida por los estados de Chiapas, Guerrero, Oaxaca y Veracruz. 
MÉXICO: Chiapas: (Pinto, 1991). Guerrero: Acahuizotla (Champion, 1892); Acapulco (Champion, 1892); $16 \mathrm{mi}$. al S de Chilpancingo (Werner, 1958); Hacienda de la Imagen (Champion, 1892); Tierra Colorada (Champion, 1892). Oaxaca: entre Tequesistlán y Toloapan (Selander \& Agafitei, 1982). Veracruz: Xalapa (Champion, 1892; Werner, 1958).

\section{Epicauta (Macrobasis) ochrea (LeConte, 1853)}

Lytta ochrea LeConte, 1853: 342 (*)

TERRA TYPICA: "New Braunfels, Texas". La serie típica consta de un único ejemplar, $n^{\circ} 4987$ en la colección del Museum of Comparative Zoology (Harvard University, EEUU) (fide Werner, 1945). Macrobasis ochrea (LeConte, 1853): Horn, 1873: 91 (*)

Cantharis protarsalis Dugès, 1877: $62(*)$

TERRA TYPICA: “... en una casa de Guanajuato...”. Holotipo posiblemente depositado en la colección del Instituto de Biología (Universidad Nacional Autónoma de México) (fide Werner, 1945). Macrobasis protarsalis (Dugès, 1877): Dugès, 1886a: $582(*)$

Cantharis ochrea (LeConte, 1853): Beauregard, 1890: 496 (*)

Epicauta moniliformis Dillon, 1950: $103(*)$

TERRA TYPICA: "Palmerlee, Arizona". Holotipo en el Museum of

Zoology of the University of Michigan (EEUU) (fide Dillon, 1950).

Epicauta ochrea (LeConte, 1853): Werner, 1945: 495 (*)

COMENTARIOS TAXONÓMICOS: Incluida en el grupo de E. ochrea (Werner, 1954b; Pinto, 1991) y en el subgénero Gnathospasta por Werner et al. (1966).

DISTRIBUCIÓN GEOGRÁFICA: Especie distribuida por el norte y centro de México y el sur de los EEUU (Pinto, 1991). En México se ha citado en los estados de Guanajuato y Sonora. Werner et al. (1966) no consideran el registro de Sonora.

MÉXICo: Guanajuato: Guanajuato (Dugès, 1877, 1889e sub M. protarsalis; Champion, 1892; Werner et al., 1966). Sonora: Norte de Sonora (Champion, 1892).

\section{Epicauta (Macrobasis) pacifica Maydell, 1934}

Epicauta pacifica Maydell, 1934: $330(*)$

TERRA TYPICA: “...Guadalajara, Jalisco, Mexico...”. Holotipo en la colección del Museum of Comparative Zoology (Harvard University, EEUU) (fide Maydell, 1934)

COMEnTARIOS TAXonÓMICOs: Pinto (1991) la incluye en el grupo de E. funesta sensu Werner (1954b) dentro del subgénero Macrobasis, y señala que posiblemente se trate de una variante de coloración de E. croceicincta.

DisTRIBUCIÓN GEOGRÁFICA: Especie endémica de México (Pinto, 1991), conocida únicamente de Jalisco y Nayarit.

MÉXICo: Jalisco: Guadalajara (Maydell, 1934); Laguna de Chapala (Pinto, 1991). Nayarit: Tepic (Maydell, 1934).

\section{Epicauta (Macrobasis) polingi Werner, 1944}

Epicauta polingi Werner, 1944: 71 (*)

TERRA TYPICA: "Davis Mts., Texas". Holotipo no 26071, en la colección del Museum of Comparative Zoology (Harvard University, Cambridge, EEUU) (fide Werner, 1944).

Epicauta luteola Dillon, 1952: 411 (*)

TERRA TYPICA: "Presidio...".

COMENTARIOS TAXONÓMICOS: Pinto (1991) establece la sinonimia de E. luteola con E. polingi tras revisar el holotipo. Incluida en el grupo de $E$. diversicornis (Werner, 1949b; Selander, 1982; Pinto, 1991). Selander (1954b) indica que los ejemplares de Chihuahua y Durango difieren de los ejemplares típicos y podrían representar sendas subespecies. Werner et al. (1966) la consideran parte del subgénero Gnathospasta.

DISTRIBUCIÓN GEOGRÁFICA: Especie distribuida por el norte y este de México y el sur de los EEUU (Pinto, 1991). Werner (1945) indica que Horn (1885) se refirió a esta especie erróneamente bajo el nombre de $M$. linearis LeConte, 1858. En México se ha citado en los estados de Chihuahua, Coahuila, Durango, Nuevo León y Tamaulipas. Vaurie (1950) indica que algunas de las citas de Champion (1892) de E. diversicornis corresponden a $E$. polingi.

MÉxICo: Chihuahua: 20 mi. al S de Ciudad Juárez (Selander, 1954b); Chihuahua (Champion, 1892 sub E. diversicornis; Vaurie, 1950); $6 \mathrm{mi}$ al $\mathrm{N}$ de Meoqui (Werner et al., 1966); Samalayuca (Vaurie, 1950); Delicias (Vaurie, 1950); 10 mi. al S de Delicias (Vaurie, 1950); 20 mi. al SO de Camargo (Vaurie, 1950); Ojo Laguna (Vaurie, 1950); Catarinas (Vaurie, 1950). Coahuila: La Gloria, al S de Monclova (Vaurie, 1950); Paila (Vaurie, 1950); Guadalupe (Vaurie, 1950); 5 mi. al N de Saltillo (Vaurie, 1950); Saltillo (Werner et al., 1966). Durango: $35 \mathrm{mi}$. al S de El Entronque (Selander, 1954b; Werner et al., 1966); Pedricena (Vaurie, 1950; Werner et al., 1966); Ventanas (Champion, $1892 \mathrm{sub}$ E. diversicornis; Vaurie, 1950); Villa Lerdo (Champion, 1892 sub E. diversicornis; Vaurie, 1950). Nuevo León: Apodaca (Selander, 1982); Monterrey (Werner, 1949a; Vaurie, 1950; Werner et al., 1966). Tamaulipas: (Pinto, 1991)

Epicauta (Macrobasis) prosopidis Werner, 1973

Epicauta (Macrobasis) prosopidis Werner, 1973: $461(*)$

Terra typica: "Mexico, Km. 739, Hwy. 45 [from Zacatecas] to Durango...". Holotipo en la colección del Museum of Comparative Zoology (Harvard University, Cambridge, EEUU) (fide Werner, 1973).

Comentarios taXonómicos: Incluida en el grupo de E. prosopidis (Werner, 1973; Pinto, 1991).

DisTRIBUCIÓN GEOGRÁFICA: Endemismo mexicano (Pinto, 1991), sólo conocido de Durango.

MÉxICo: Durango: Mexico, Km. 739, Hwy. 45 [from Zacatecas] to Durango, cerca deVicente Guerrero (Werner, 1973).

Epicauta (Macrobasis) punctum (Dugès, 1869)

Cantharis punctum Dugès, 1869c: 158 (*)

TERRA TYPICA: No señalada, aunque la localidad mencionada por Dugès en un manuscrito inédito, que reproduce Zaragoza-Caballero (1999), es Cotija. El tipo está perdido según Pinto (1991).

Epicauta punctum (Dugès, 1869): Dugès, 1886a: $582\left(^{*}\right)$

COMENTARIOS TAXONÓMICOS: Beauregard (1890) y Borchman (1917) la incluyen en el subgénero nominal, mientras que Pinto (1991) la incluye en el grupo de $E$. funesta sensu Werner (1954b) del subgénero Macrobasis. Epicauta unicalcarata parece ser un sinónimo de E. punc- 
tum, pero el tipo de E. punctum está aparentemente perdido y hasta que no se designe neotipo este punto no puede comprobarse (Pinto, 1991). Dugès (1869c) señala el nombre no disponible Cantharis punctum Deyroles [sic].

DistribuCión GEOGRÁFICA: Especie endémica de México (Pinto, 1991), localizada en los estados de Durango, Guerrero, Jalisco, Michoacán, Morelos, Puebla, Oaxaca y Veracruz. Pinto (1991) únicamente señala la presencia de la especie en Morelos y Oaxaca.

MÉXICO: Durango: Ventanas (Champion, 1892; Vaurie, 1950). Guerrero: Mochitlán (Champion, 1892; Vaurie, 1950). Jalisco: Guadalajara (Champion, 1892; Vaurie, 1950). Michoacán: Cotija (Zaragoza-Caballero, 1999). Morelos: (Pinto, 1991). Puebla: Izúcar de Matamoros (Champion, 1892; Vaurie, 1950). Oaxaca: La Parada (Champion, 1892; Vaurie, 1950). Veracruz: Veracruz (Dugès, 1889e; Champion, 1892; Vaurie, 1950).

Epicauta (Macrobasis) purpurea (Horn, 1885)

Macrobasis purpurea Horn, 1885: 108 (*)

TERRA TYPICA: "Arizona". La serie típica consta de un único ejemplar, $n^{\circ} 8086$ (fide Werner, 1945), inicialmente en la colección de la Academy of Natural Sciences of Philadelphia, posteriormente transferido al Museum of Comparative Zoology de Harvard (EEUU) Cantharis purpurea (Horn, 1885): Champion, 1899: $185\left({ }^{*}\right)$ Epicauta purpurea (Horn, 1885): Werner, 1945: 504 (*)

COMENTARIOS TAXONÓMICOS: Incluida en el grupo de E. prosopidis (Werner, 1973; Pinto, 1991), dentro del subgénero Gnathospasta por Werner et al. (1966) y luego en Macrobasis por Werner (1973) y Pinto (1991).

DisTRIBUCIÓN GEOGRÁFICA: Especie distribuida por el norte de México y Arizona (EEUU) (Pinto, 1991). En México se ha citado en los estados de Durango, Sinaloa y Sonora.

MÉXICO: Durango: Ventanas (Champion, 1892; Vaurie, 1950; Werner et al., 1966); Palos Colorados [cerca de Ciudad Durango] (Vaurie, 1950; Werner et al., 1966). Sinaloa: (Pinto, 1991). Sonora: 8 mi. al O de Coyote (Werner et al., 1966).

\section{Epicauta (Macrobasis) segmenta (Say, 1824)}

\section{Lytta segmenta Say, 1824b: 303 (*)}

TERRA TYPICA: “...near Purgatory River of the Arkansa, ... the First Fork", originalmente; "Fort Hayes, Kansas", tras designación de neotipo por Werner (1945) en la colección del Museum of Comparative Zoology (Harvard University, Cambridge, EEUU).

Lytta segmentata Say, 1824b: LeConte, 1853: 342 (enmienda injustificada) (*)

Apterospasta segmenta(ta) (Say, 1824): LeConte, 1862: $272(*)$

Macrobasis segmentata (Say, 1824): Horn, 1873: 93 (*)

Macrobasis cinctothorax Dugès, 1889e: $56(*)$

TERRA TYPICA: “Chihuahua?”. Vázquez \& Zaragoza (1979) indican como tipo el ejemplar $n^{\circ} 2878$ (IBUNAM 023) de la colección del Instituto de Biología de la Universidad Nacional Autónoma de México.

Cantharis segmentata (Say, 1824): Champion, 1899: 187 (*)

Epicauta segmenta (Say, 1824): Werner, 1945: 490 (*)

COMENTARIOS TAXONÓMICOS: En la obra de ZaragozaCaballero (1999: 22) se incluye el error tipográfico Macrobasis cinctotorax. Beauregard (1890), Champion
(1892) y Werner (1945) consideran a E. valida (LeConte, 1858) como una variedad de E. segmenta y Borchmann (1917) la incluye en su sinonimia, pero Selander \& Mathieu (1969) y otros autores difieren de esta opinión y tratan a $E$. valida como un taxon independiente (que no se ha encontrado en México). Incluida en el grupo de $E$. albida (Selander \& Mathieu, 1969; Pinto, 1991).

DISTRIBUCIÓN GEOGRÁFICA: Especie ampliamente distribuida por el norte de México y en los EEUU (Pinto, 1991). En México se ha citado en los estados de Chihuahua, Coahuila, Durango, Querétaro Sinaloa y Sonora.

MÉxICo: Chihuahua: Cañón de Bachimba, 27 mi. al S de Chihuahua (Selander \& Mathieu, 1969); 60 mi. al NO de Casas Grandes (Selander \& Mathieu, 1969); Catarinas (Vaurie, 1950; Selander \& Mathieu, 1969); Chihuahua (Dugès, 1889e sub $M$. cinctothorax; Champion, 1892; Zaragoza-Caballero, 1999); Delicias (Vaurie, 1950; Selander \& Mathieu, 1969); $10 \mathrm{mi}$. al S de Delicias (Vaurie, 1950; Selander \& Mathieu, 1969); 12 mi. al NO de Gran Morelos (Selander \& Mathieu, 1969); 13 mi. al S de Hidalgo del Parral (Selander \& Mathieu, 1969); Hidalgo del Parral (Vaurie, 1950); Santa Clara (Champion, 1892; Vaurie, 1950; Selander \& Mathieu, 1969); Valle de Olivos (Vaurie, 1950; Selander \& Mathieu, 1969); 1 mi. al N de Villa Matamoros (Selander \& Mathieu, 1969). Coahuila: Ojo de Agua, Sierra de Tlahualillo (Selander \& Mathieu, 1969). Durango: Ciudad Lerdo (Champion, 1892; Vaurie, 1950; Werner et al., 1966; Selander \& Mathieu, 1969); Carretera 45, cerca de Mimbrera (Selander \& Mathieu, 1969); Tlahualillo (Selander \& Mathieu, 1969). Querétaro: Rancho Espíndola, $4 \mathrm{~km}$ al N de San Juan del Río (García-París et al., 2005). Sinaloa: Escuinapa de Hidalgo (Vaurie, 1950; Werner et al., 1966; Selander \& Mathieu, 1969). Sonora: 4 mi. al O de Guasabas (Selander \& Mathieu, 1969); Guaymas (Selander \& Mathieu, 1969); 40 mi. al N de Guaymas (Selander \& Mathieu, 1969); Hermosillo (Selander \& Mathieu, 1969); Naco (Vaurie, 1950; Selander \& Mathieu, 1969).

\section{Epicauta (Macrobasis) selanderorum Werner, 1958}

Epicauta selanderorum Werner, 1958: 6 (*)

TERRA TYPICA: "16 mi. S.E. of Lagos de Moreno, Jalisco, Mexico, 6700 ', ...". Holotipo en la colección del Museum of Comparative Zoology (Harvard University, Cambridge, EEUU) (fide Werner, 1958).

COMENTARIOS TAXONÓMICOS: Werner (1958) indica que, aunque la descripción no coincide, esta especie tal vez pudiera corresponder a E. labialis. Incluida en el grupo de E. uniforma (Werner, 1954b; Pinto, 1991).

Distribución GEOGRÁFICA: Especie endémica de México (Pinto, 1991). Conocida de los estados de Jalisco, Michoacán y Querétaro.

MÉxICo: Jalisco: $16 \mathrm{mi}$. al SE de Lagos de Moreno (Werner, 1958). Michoacán: (Pinto, 1991). Querétaro: (Pinto, 1991).

\section{Epicauta (Macrobasis) stigmata (Dugès, 1869)}

Cantharis stigmata Dugès, 1869c: 159 (*)

TERRA TYPICA: “...Guanajuato”. Supuesto holotipo figurado erróneamente por Dugès (1881: lám. 1, figs. 10,10a), que corresponde en 
realidad a L. erythrothorax. Vázquez \& Zaragoza (1979) indican como tipo el ejemplar $n^{\circ} 803$ (IBUNAM 003) de la colección del Instituto de Biología de la Universidad Nacional Autónoma de México.

Lytta neglecta Haag-Rutenberg, 1880: 54 (*)

TERRA TYPICA: "Mexico" originalmente; "Mex.” tras designación de lectotipo (Pinto, 1982) en la colección del British Museum of Natural History (Londres). La serie típica consta de otros dos ejemplares, uno de ellos (hembra) correspondiente a E. mixta (fide Pinto, 1982).

Epicauta stigmata (Dugès, 1869): Dugès, 1886a: 582 (*)

Epicauta intermedia Dugès, 1889e: 69 (non Lytta intermedia Haag Rutenberg, 1880 = Epicauta intermedia) (*)

TERRA TYPICA: "Vera Cruz".

Epicauta neglecta (Haag-Rutenberg, 1880): Beauregard, 1890: 508 (*) Cantharis neglecta (Haag-Rutenberg, 1880): Champion, 1899: 183 (*) Macrobasis stigmata (Dugès, 1869): Borchmann, 1917: 88 (*)

COMENTARIOS TAXONÓMICOS: Incluida en el grupo de E. uniforma (Werner, 1954b; Pinto, 1991). Beauregard (1890) y Borchmann (1917) incluyeron a L. neglecta en el subgénero nominal. Horn (1885) y Beauregard (1890) trataron equivocadamente a $C$. stigmata como variedad de E. cinerea (Forster, 1771). Epicauta stigmata y E. neglecta se han venido tratando como especies independientes, hasta que Pinto (1982) estableció su sinonimia. Werner (1958) y Pinto (1991) añaden que E. uniforma podría ser en realidad una variante geográfica septentrional de E. stigmata. Dugès (1874b, 1886a, 1889e) utiliza el nombre no disponible Epicauta nigritarsis Chevrolat y el propio Dugès (1881) usa el nombre no disponible $E$. stigmata Sturm. Champion (1899) recoge el nombre no disponible Epicauta anilis Sturm, 1843: 175.

DisTRIBUCIÓN GEOGRÁFICA: Endemismo mexicano (Pinto, 1991). Ampliamente distribuida por los estados de Distrito Federal, Guanajuato, Jalisco, México, Michoacán, Morelos, Puebla, Querétaro y Tlaxcala. Champion (1892), seguido por Borchmann (1917) y Vaurie (1950), consideran que las citas de Dugès (1889e) de E. intermedia y de E. nigritarsis (LeConte, 1853) corresponden a E. stigmata. Werner (1958) señala que las citas de Vaurie (1958) de E. stigmata de Durango y Zacatecas corresponden a E. uniforma y que la cita de Champion (1892) de Saltillo (Coahuila), recogida después por Vaurie (1950), tampoco corresponde a E. stigmata. Pinto (1991) señala que parte de las citas de Haag-Rutenberg (1880) y todas las de Champion (1892) de E. neglecta corresponden a E. mixta.

MÉxICo: Aguascalientes: Aguascalientes (Champion, 1892; Vaurie, 1950). Distrito Federal: Guadalupe (Vaurie, 1950); Tlalpan (Werner, 1958). Guanajuato: Guanajuato (Dugès, 1869c; Champion, 1892; Zaragoza-Caballero, 1999); Irapuato (Champion, 1892; Vaurie, 1950); León (Werner, 1958); 5 mi. al N de Salamanca (Werner, 1958); Tupátaro (Zaragoza-Caballero, 1999). Jalisco: Chapala (Werner, 1958); Guadalajara (Champion, 1892; Vaurie, 1950); Jiquilpan (Werner, 1958); Lagos de Moreno (Werner, 1958); 9 mi. al N de Mazamitla (Werner, 1958); El Molino (Werner, 1958); 10 km al N de Tepatitlán (Werner, 1958). México: Atlacomulco
(Werner, 1958); Toluca (Werner, 1958); 20 mi. al N de Toluca (Werner, 1958); Volcán Ixtaccihuatl (Champion, 1892; Vaurie, 1950). Michoacán: Chavinda (Werner, 1958); Jacona (Werner, 1958); Morelia (Zaragoza-Caballero, 1999); Pátzcuaro (Werner, 1958); $10 \mathrm{~km}$ al E de Quiroga (Werner, 1958); $17 \mathrm{~km}$ al E de Quiroga (Werner, 1958); Tacámbaro (Champion, 1892); Zamora (Werner, 1958). Morelos: $8 \mathrm{~km}$ al E de Cuernavaca (Pinto, 1991); Tepoztlán (Werner, 1958). Puebla: Izúcar de Matamoros (Champion, 1892; Vaurie, 1950); Puebla (Werner, 1958). Querétaro: cerca de Hacienda Balvanera, 10 $\mathrm{km}$ al O de Querétaro (Werner, 1958); Querétaro (Champion, 1892). Tlaxcala: 4 mi. al NO de Huamantla (Werner, 1958). Veracruz: Veracruz (Dugès, $1889 \mathrm{e}$ sub E. intermedia; Champion, 1892).

Epicauta (Macrobasis) sublineata (LeConte, 1854)

Lytta sublineata LeConte, 1854a: 447 (*)

TERRA TYPICA: No indicada originalmente; "Vicinity of Eagle Pass" en la etiqueta del holotipo (fide Selander \& Mathieu, 1969). Holotipo $\mathrm{n}^{\circ} 4979$, en la colección del Museum of Comparative Zoology (Harvard University, Cambridge, EEUU) (fide Werner, 1945; Selander \& Mathieu, 1969)

Macrobasis sublineata (LeConte, 1854): Horn, 1873: 94 (*)

Macrobasis megacephala Champion, 1892: 402 (*)

Terra tyPICA: "MeXico, Monclova in Coahuila". Dos sintipos, posiblemente en el British Museum of Natural History (Londres) (fide Werner, 1945; Selander \& Mathieu, 1969).

Cantharis megacephala (Champion, 1892): Champion, 1899: 182 (*) Epicauta sublineata (LeConte, 1854): Werner, 1945: 491 (*) $^{*}$

Epicauta reinhardi Dillon, 1952: 413 (*)

TERRA TYPICA: "Dimmit Co. Texas...". Holotipo en la colección de la Texas Agricultural and Mechanical University (EEUU) (fide Selander \& Mathieu, 1969).

COMEnTARIOS TAXONÓMICOS: Selander \& Mathieu (1969) destacan que Horn (1885) indicó erróneamente que la descripción de E. sublineata se basó en un ejemplar desgastado de E. longicollis, de forma que las sinonimias posteriores de estas dos especies (Champion, 1892 ) son erróneas. Selander \& Mathieu (1969) establecen la sinonimia de E. reinhardi con E. sublineata. Incluida en el grupo de E. albida (Selander \& Mathieu, 1969; Pinto, 1991).

DistriBUCIÓN GEOGRÁFICA: Especie extendida por el noreste de México y Texas (Pinto, 1991). En México se ha citado en los estados de Coahuila, Nuevo León y Tamaulipas.

MÉXICo: Coahuila: 12 mi. al N de Hermanas (Selander \& Mathieu, 1969); 12 mi. al NO de Jiménez (Selander \& Mathieu, 1969); Monclova (Champion, 1892; Vaurie, 1950; Selander \& Mathieu, 1969). Nuevo León: Apodaca (Selander \& Mathieu, 1969); El Cercado (Selander \& Mathieu, 1969); 7 mi. al NE de Monterrey (Selander \& Mathieu, 1969); Pesquería (Selander \& Mathieu, 1969); Sabinas Hidalgo (Selander \& Mathieu, 1969); $17 \mathrm{~km}$ al N de Sabinas Hidalgo (Selander \& Mathieu, 1969). Tamaulipas: Ciudad Victoria (Selander \& Mathieu, 1969); 2 mi. al N de Ciudad Victoria (Selander \& Mathieu, 1969); Güemes (Selander \& Mathieu, 1969); $10 \mathrm{~km}$ al N de Padilla (Selander \& Mathieu, 1969); Sierra de San Carlos (Selander \& Mathieu, 1969). 
Epicauta (Macrobasis) tenella (LeConte, 1858)

Lytta tenella LeConte, 1858a: 23 (*)

TERRA TYPICA: "Llano Estacado...". Lectotipo no 4988 designado por

Werner (1945) en la colección del Museum of Comparative Zoology (Harvard University, Cambridge, EEUU).

Macrobasis tenella (LeConte, 1858): Horn, 1873: 94 (*)

Epicauta merkeliana Horn, 1891: 43 (*)

TERRA TYPICA: “Arizona, probably Fort Whipple...”. La serie típica consta de un único ejemplar (Horn, 1891), nº 8091 (fide Werner,

1945), inicialmente en la colección de la Academy of Natural

Sciences of Philadelphia, posteriormente transferido al Museum of Comparative Zoology de Harvard (EEUU).

Cantharis merkeliana (Horn, 1891): Champion, 1899: 182 (*)

Epicauta tenella (LeConte, 1858): Werner, 1945: 493 (*)

COMENTARIOS TAXONÓMICOS: Borchmann (1917) incluye a $E$. merkeliana en el subgénero nominal y la trata como especie independiente de $E$. (M.) tenella. Werner (1945) también considera a E. merkeliana una especie distinta de $E$. tenella. Incluida en el grupo de $E$. tenella (Werner, 1954b; Pinto, 1991). Werner et al. (1966) la consideran parte del subgénero Gnathospasta.

DisTRIBUCIÓN GEOGRÁFICA: Presente en el norte de México y el sur de los EEUU (Pinto, 1991). En México se ha citado en los estados de Baja California Sur, Chihuahua, Durango y Sonora.

MÉxICo: Baja California Sur: (Werner et al., 1966; Pinto, 1991). Chihuahua: 20 mi. al S de Ciudad Juárez (Selander, 1954b); Delicias (Vaurie, 1950); $10 \mathrm{mi}$. al S de Delicias (Vaurie, 1950). Durango: $35 \mathrm{mi}$. al S de El Entronque (Selander, 1954b). Sonora: Ciudad Obregón (Werner et al., 1966); Guaymas (Werner et al., 1966); Hermosillo (Werner et al., 1966); Sonoyta (Selander, 1958).

Epicauta (Macrobasis) tenuemarginata Werner, 1958 Epicauta tenuemarginata Werner, 1958: $13(*)$

TERRA TYPICA: "Patzcuaro, Michoacan, Mexico, 6500', ...". Holotipo en la colección del Museum of Comparative Zoology (Harvard University, Cambridge, EEUU) (fide Werner, 1958). Paratipos en diversas colecciones, incluida la del Instituto de Biología de la Universidad Nacional Autónoma de México (fide Werner, 1958).

COMENTARIOS TAXONÓMICOS: Conservada en el grupo de E. uniforma sensu Werner (1954b) por Werner (1958) y Pinto (1991).

DisTRIBUCIÓN GEOGRÁFICA: Endemismo mexicano (Pinto, 1991). Especie únicamene citada en Jalisco y Michoacán. Werner (1958) indica que Dugès (1889) incluyó ejemplares de esta especie de Michoacán bajo la denominación de E. stigmata.

MÉXICO: Jalisco: $96 \mathrm{~km}$ al SO de Guadalajara (Werner, 1958); Guadalajara (Werner, 1958); 9 mi. al N de Mazamitla (Werner, 1958); El Molino (Werner, 1958). Michoacán: Pátzcuaro (Werner, 1958); $17 \mathrm{~km}$ al E de Quiroga (Werner, 1958).

Epicauta (Macrobasis) tenuicornis (Champion, 1892) Macrobasis tenuicornis Champion, 1892: 400 (*)

TeRra tyPica: "Mexico, Chilpancingo...". Dos sintipos en la colección del British Museum of Natural History (Londres).
Epicauta tenuicornis (Champion, 1892): Blackwelder, 1945: 483 (*)

COMENTARIOS TAXONÓMICOS: Incluida en el grupo de E. diversicornis (Werner, 1949b; Pinto, 1991).

DistribuCión GEOGRÁFICA: Especie endémica de México (Pinto, 1991). Localizada en los estados de Guerrero, Michoacán, Morelos y Puebla.

MÉxICo: Guerrero: Chilpancingo (Champion, 1892). Michoacán: (Pinto, 1991). Morelos: Cañon del Lobo [Cañón de Lobos] (Pinto, 1991). Puebla: (Pinto, 1991).

\section{Epicauta (Macrobasis) tenuilineata (Horn, 1894)}

Macrobasis tenuilineata Horn, 1894: 436 (*)

TerRa typica: "Sonora, Mexico and San Jose del Cabo". Werner (1945) señala que los tipos podrían encontrarse en la California Academy of Sciences (San Francisco, EEUU).

Cantharis tenuilineata (Horn, 1894): Champion, 1899: 189 (*)

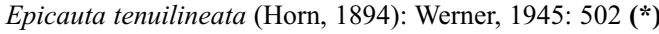

COMENTARIOS TAXONÓMICOS: Especie incluida en el grupo de E. tenuis (Selander \& Mathieu, 1969; Pinto, 1991).

DisTRIBUCIÓN GEOGRÁFICA: Distribuida por el noroeste de México y suroeste de los EEUU (Pinto, 1991). Según Pinto (1991), en México sólo se ha citado en Baja California Sur, aunque Horn (1894) y Werner (1945) también la incluyen en Sonora.

MÉXICo: Baja California Sur: San José del Cabo (Horn, 1894; Werner, 1945). Sonora: Ciudad Obregón (Werner et al., 1966); Guaymas (Werner et al., 1966); Hermosillo (Werner et al., 1966); Puerto Libertad (Werner et al., 1966); Sonora (Horn, 1894; Werner, 1945)

Epicauta (Macrobasis) terminata (Dugès, 1869)

Cantharis terminata Dugès, 1869c: 157 (*)

TERRA TYPICA: No indicada, aunque en la redescripción de Dugès (1889e: 78) señala "Vera Cruz". Vázquez \& Zaragoza (1979) indican como tipo el ejemplar no 801 (IBUNAM 006) de la colección del Instituto de Biología de la Universidad Nacional Autónoma de México.

Epicauta terminata (Dugès, 1869): Dugès, 1886a: 582 (*)

COMENTARIOS TAXONÓMICOS: Incluida en el subgénero nominal por Champion (1892), seguido por Borchmann (1917), mientras que Pinto (1991) sigue a Werner (1954b) y la mantiene en el grupo de E. funesta, del subgénero Macrobasis. Epicauta apicalis parece ser una variante de color de E. terminata (Pinto, 1991). Dugès $(1869 \mathrm{c})$ señala el nombre no disponible Pyrota terminata Sturm, 1843: 174.

DisTRIBUCIÓN GEOGRÁFICA: Especie exclusiva de México (Pinto, 1991), ampliamente distribuida en los estados de Colima, Guerrero, Jalisco, Michoacán, Oaxaca, Puebla y Veracruz.

MÉXICo: Colima: (Pinto, 1991). Guerrero: Acaguizotla (Champion, 1892); Mexcala (Selander, 1954b; Werner, 1958); Mochitlán (Champion, 1892). Jalisco: Guadalajara (Champion, 1892). Michoacán: (Vázquez \& Zaragoza, 1979; Pinto, 1991; Zaragoza-Caballero, 1999). Oaxaca: (Pinto, 1991). Puebla: 
Izúcar de Matamoros (Champion, 1892); Puebla (Champion, 1892). Veracruz: Veracruz (Champion, 1892).

\section{Epicauta (Macrobasis) texana Werner, 1944}

Epicauta texana Werner, 1944: 73 (*)

TERRA TYPICA: "Davis Mts., Texas". Holotipo en la colección de The Ohio State University (EEUU).

COMENTARIOS TAXONÓMICOS: Incluida en el grupo de E. albida (Selander \& Mathieu, 1969; Pinto, 1991).

DisTRIBUCIÓN GEOGRÁFICA: Localizada en el norte de México y sur de los EEUU (Pinto, 1991). En México se ha citado únicamente en el estado de Durango.

MÉXICO: Durango: Bermejillo (Selander \& Mathieu, 1969).

\section{Epicauta (Macrobasis) tripartita Champion, 1892}

Epicauta tripartita Champion, 1892: 421 (*)

TERRA TYPICA: "MeXICO, Ventanas in Durango...". Tres sintipos en el British Museum of Natural History (Londres) (fide Vaurie, 1950). Cantharis tripartita (Champion, 1892): Champion, 1899: 190 (*)

COMENTARIOS TAXONÓMICOS: Incluida en el subgénero nominal por Champion (1892) y Borchmann (1917), mantenida en en el subgénero Macrobasis dentro del grupo de E. uniforma sensu Werner (1954b) por Pinto (1991).

Distribución GEOGRÁfiCA: Especie endémica de México (Pinto, 1991). Localizada en los estados de Chihuahua, Durango, Nayarit y Sinaloa.

MÉXICo: Chihuahua: (Pinto, 1991). Durango: Ventanas (Champion, 1892; Vaurie, 1950; Werner, 1958). Nayarit: Tepic (Werner, 1958). Sinaloa: (Pinto, 1991).

\section{Epicauta (Macrobasis) triquetra Werner, 1958 \\ Epicauta triquetra Werner, 1958: 15 (*) \\ TERra tYPICA: "5 mi. E. C(iudad) del Maiz, (San Luis Potosi), Mex(ico), 4700' ...". Holotipo en la colección del Museum of Comparative Zoology (Harvard University, Cambridge, EEUU) (fide Werner, 1958).}

COMENTARIOS TAXONÓMICOS: Incluida en el grupo de E. uniforma (Werner, 1954b; Pinto, 1991).

DisTRIBUCIÓN GEOGRÁFICA: De esta especie únicamente se conoce el holotipo (Pinto, 1991), capturado en San Luis Potosí. 1958).

MÉxICo: San Luis Potosí: Ciudad del Maíz (Werner,

Epicauta (Macrobasis) unicalcarata Champion, 1892 Epicauta unicalcarata Champion, 1892: 412 (*)

TERra TYPICA: "MeXICO, Amula in Guerrero". Holotipo en la colección del British Museum of Natural History (Londres) (fide Pinto, 1982).

Cantharis unicalcarata (Champion, 1892): Champion, 1899: 190 (*)

COMENTARIOS TAXONÓMICOS: Epicauta unicalcarata parece ser un sinónimo de E. punctum, pero el tipo de $E$. punctun está aparentemente perdido y hasta que no se designe neotipo este punto no puede establecerse (Pinto, 1991). Incluida en el subgénero nominal por Champion
(1892) y Borchmann (1917), Pinto (1982, 1991) la incluye en Macrobasis, dentro del grupo de E. funesta sensu Werner (1954b).

DistribuCión GEOGRÁfiCA: Especie endémica de México (Pinto, 1991), sólo conocida de Guerrero.

MÉxICo: Guerrero: Amula (Champion, 1892; Pinto, 1982).

\section{Epicauta (Macrobasis) uniforma Werner, 1944}

Epicauta uniforma Werner, 1944: 67 (*)

TERRA TYPICA: "Ramah, Arizona". Holotipo no 26581, en la colección del Museum of Comparative Zoology (Harvard University, Cambridge, EEUU).

COMENTARIOS TAXONÓMICOS: Incluida en el grupo de E. uniforma (Werner, 1954b; Pinto, 1991) y en el subgénero Gnathospasta por Werner et al. (1966). Pinto (1991) indica que E. uniforma podría representar una variante geográfica septentrional de E. stigmata.

DisTRIBUCIÓN GEOGRÁFICA: Especie presente en el norte de México y sur de los EEUU (Pinto, 1991). En México se ha citado en los estados de Chihuahua, Durango, Sonora y Zacatecas. Werner (1958) señala que las citas de Vaurie (1958) de E. stigmata de Durango y Zacatecas corresponden a E. uniforma.

MÉXICo: Chihuahua: Ciudad Juárez (Selander, 1954b). Durango: San Lucas (Vaurie, 1950 sub E. stigmata); Yerbanís, Distrito de Cuencamé (Vaurie, 1950 sub E. stigmata); Pedricena (Vaurie, 1950 sub E. stigmata). Sonora: (Pinto, 1991). Zacatecas: Fresnillo (Vaurie, 1950 sub E. stigmata; Werner, 1958; Werner et al., 1966); 19 mi. al S de Sombrerete (Selander, 1954b; Werner, 1958).

\section{Epicauta (Macrobasis) virgulata (LeConte, 1866)}

Macrobasis virgulata LeConte, 1866: 156 (*)

TERRA TYPICA: "Cape San Lucas" en la descripción original; "San Jose del Cabo, Lower California" tras designación de lectotipo. Lectotipo, $n^{\circ} 4981$, designado por Werner (1945) en la colección del Museum of Comparative Zoology (Harvard University, Cambridge, EEUU).

Epicauta virgulata (LeConte, 1866): Werner, 1945: 512 (*)

COMENTARIOS TAXONÓMICOS: Incluida en el grupo de E. virgulata (Werner, 1954b; Pinto, 1991). Werner (1945) trató a E. hirsutipubescens como sinónimo de $E$. virgulata, pero posteriormente restableció su estatus específico (Werner, 1949b). Incluida en el subgénero Gnathospasta por Werner et al. (1966).

DisTRIBUCIÓN GEOGRÁFICA: Especie distribuida por el noroeste de México y suroeste de los EEUU (Pinto, 1991). En México se ha citado en los estados de Baja California, Baja California Sur, Sinaloa y Sonora.

MÉxICo: Baja California: San Quintín (Werner, 1949b); 10 mi. al S de Cataviña (Werner, 1949b); Santo Domingo (Werner, 1949b). Baja California Sur: Cabo San Lucas (LeConte, 1866; Horn, 1873, 1894; Beauregard, 1890); San José del Cabo (Werner, 1945); San José de Comondú (Werner, 1949b); "Coyote Cove" en Bahía Concepción (Werner, 1949b); 5 mi. al SO de San Miguel, Santo Domingo (Werner, 
1949b); San Venancio (Werner, 1949b); El Triunfo (Werner, 1949b); La Paz (Werner, 1949b); Todos Santos (Werner, 1949b). Sinaloa: Los Mochis (Werner, 1949b; Werner et al., 1966). Sonora: Ciudad Obregón (Werner et al., 1966); Hermosillo (Werner et al., 1966); Navajoa (Werner et al., 1966); Nogales (Werner, 1945).

\section{Género Linsleya MacSwain, 1951}

Linsleya MacSwain, 1951a: 58 (*)

ESPECIE TIPO: Lytta convexa LeConte, 1853, por designación original.

CONTENIDO Y DISTRIBUCIÓN: El género cuenta con 5 especies exclusivas de América del Norte, distribuidas desde el norte de México al suroeste del Canáda (Pinto \& Bologna, 1999). Dentro del género se diferencian dos subgéneros, Linsleya y Linsleyina Selander, 1955, de los que sólo el primero se ha encontrado en México. Linsleya (Linsleyina) californica Selander, 1955 y Linsleya (Linsleyina) sphaericollis (Say, 1824) se han localizado en California (EEUU), muy cerca de la frontera por lo que su presencia en México es probable.

Subgénero Linsleya MacSwain, 1951

Linsleya MacSwain, 1951a: 58 (*)

ESPECIE TIPO: Lytta convexa LeConte, 1853, por designación original.

CONTENIDO Y DISTRIBUCIÓN: En México se ha citado la presencia de $L$. convexa y L. suavissima.

Linsleya (Linsleya) convexa (LeConte, 1853)

Lytta convexa LeConte, 1853: 336 (*)

TERRA TYPICA: "Mexican Boundary...". Holotipo posiblemente en la colección del Museum of Comparative Zoology en Harvard University (Cambridge, EEUU) (fide Selander, 1955a)

Cantharis convexa (LeConte, 1853): Horn, 1873: $115\left(^{*}\right)$

Linsleya convexa (LeConte, 1853): MacSwain, 1951a: 58 (*)

DISTRIBUCIÓN GEOGRÁFICA: Especie distribuida por el noroeste de México y suroeste de los EEUU (LeConte, 1853; Selander, 1954b). En México se ha citado en Chihuahua. Según Selander (1955b) la localidad típica se situaría en la frontera entre México y Texas (EEUU).

MÉxICo: Chihuahua: $7 \mathrm{mi}$. al N de Chihuahua (Selander, 1954b, 1955a); 2 mi. al N de Sueco (Selander, 1954b, 1955a); 54 mi. S de Sueco (Selander, 1954b, 1955a).

\section{Linsleya (Linsleya) suavissima (Wellman, 1910)}

Cantharis gentilis Horn, 1883: 311 (non Cantharis flavipes var. gentilis Frivaldszky, 1877) (*)

TERRA TYPICA: "New Mexico and Arizona". Tipos posiblemente en la colección del Museum of Comparative Zoology de Harvard (EEUU).

Lytta suavissima Wellman, 1910c: 24 (nombre de substitución para Cantharis gentilis Horn, 1883) (*)

Linsleya suavissima (Wellman, 1910): MacSwain, 1951a: 58 (*)

DiSTRIBUCIÓN GEOGRÁFICA: Especie distribuida por el norte de México y suroeste de los EEUU (Vaurie, 1950). En México se ha citado en Durango.

MÉXICo: Durango: Canelas (Champion, 1892 sub C. gentilis; Vaurie, 1950; Selander, 1955a); Francisco Madero (Selander, 1955a).
Tribu Eupomphini LeConte, 1862

Eupomphae LeConte, 1862: 274 (*)

GÉNERO TIPO: Eupompha LeConte, 1858.

CONTENIDO Y DISTRIBUCIÓN: La tribu cuenta con 26 especies distribuidas en 7 géneros endémicos de la región Neártica. Todos los géneros excepto Cordylospasta Horn, 1875, que hasta el momento sólo se ha localizado en el suroeste de los Estados Unidos, se encuentran en el norte de México.

\section{Género Cysteodemus LeConte, 1851}

Cysteodemus LeConte, 1851: 158 (*)

ESPECIE TIPO: Cysteodemus armatus LeConte, 1851, por designación subsecuente de Wellman (1910b).

CONTENIDO Y DISTRIBUCIÓN: El género cuenta con dos especies exclusivas del tercio septentrional de México y del suroeste de los Estados Unidos. Ambas especies, C. armatus y $C$. wislizeni, se encuentran en México (Pinto, 1984a, c).

\section{Cysteodemus armatus LeConte, 1851}

Cysteodemus armatus LeConte, 1851: 158 (*)

TERRA TYPICA: "ad flumen Colorado..." Tipos en la colección del Museum of Comparative Zoology (Harvard University, Cambridge, EEUU) (fide Van Dyke, 1928; Pinto, 1984a).

DISTRIBUCIÓN GEOGRÁFICA: Especie distribuida por el noroeste de México y suroeste de los EEUU (Pinto, 1984a). En México se ha citado en los estados de Baja California y Sonora.

MÉxICo: Baja California: Cocopah Mts. (Pinto, 1984a); extremo norte de Laguna Salada (Pinto, 1984a); 10.3 mi. al SO de Los Médanos (Pinto, 1984a); 15 mi. al S de Palacio (Pinto, 1984a); 20 mi. al S de Palacio (Pinto, 1984a); 35 mi. al S de Río del Mayor (Pinto, 1984a); San Felipe (Pinto, 1984a); 3 mi. al N de San Felipe (Pinto, 1984a); 25 mi. al N de San Felipe (Pinto, 1984a); $35 \mathrm{mi}$. al N de San Felipe (Pinto, 1984a); $50 \mathrm{mi}$. al N de San Felipe (Pinto, 1984a); 14 mi. al S de San Felipe (Pinto, 1984a); $18 \mathrm{mi}$. al S de San Felipe (Pinto, 1984a). Sonora: 6 mi. al N de El Golfo (Pinto, 1984a); 36 mi. al NE de El Golfo (Pinto, 1984a); Puerto Peñasco (Werner et al., 1966).

\section{Cysteodemus wislizeni LeConte, 1851}

Cysteodemus wislizeni LeConte, 1851: 158 (*)

TERRA TYPICA: "Nova Mexico". Tipos en la colección del Museum of Comparative Zoology (Harvard University, Cambridge, EEUU) (fide Van Dyke, 1928; Pinto, 1984a).

Cysteodemus wislizenoi LeConte, 1851: Denier, 1935a: 176 (enmienda injustificada) $(*)$

COMENTARIOS TAXONÓMICOS: En las obras de Dugès, (1889b: 10) y Champion (1892: 369) se deslizaron los errores tipográficos Cysteodemus wisliseni y Cysteodemus wizlizeni respectivamente.

DisTRIBUCIÓN GEOGRÁFICA: Especie distribuida por el norte de México y sur de los EEUU (Pinto, 1984a). En México se ha citado en los estados de Chihuahua, Coahuila y Durango.

MÉxICo: Chihuahua: Chihuahua (Champion, 1892; Van Dyke, 1928); 25 mi. al S de Chihuahua (Pinto, 1984a); $30 \mathrm{mi}$. 
al S de Chihuahua (Selander, 1954b; Pinto, 1984a); 10 mi. al N de Ciudad Camargo (Pinto, 1984a); Entre Camargo y Parral (Spieth, 1950); 42 mi. al SO de Ciudad Camargo (Vaurie, 1950; Pinto, 1984a); Delicias (Pinto, 1984a); 27 mi. al E de Hidalgo del Parral (Pinto, 1984a); 29 km al N de Samalayuca (Pinto, 1984a); Sueco (Pinto, 1984a); 2 mi. al N de Sueco (Selander, 1954b). Coahuila: Cabos (Vaurie, 1950); Guadalupe (Vaurie, 1950; Pinto, 1984a); 70 km al N de Saltillo (Pinto, 1984a); San Pedro de las Colonias (Vaurie, 1950; Pinto, 1984a). Durango: 16 mi. al N de Bermejillo (Pinto, 1984a); Ceballos (Pinto, 1984a); 2 mi. al SE de La Zarca (Pinto, 1984a); Villa Lerdo (Champion, 1892; Van Dyke, 1928; Vaurie, 1950; Pinto, 1984a); Yermo (Pinto, 1984a).

\section{Género Eupompha LeConte, 1858}

Eupompha LeConte, 1858a: 21 (*)

ESPECIE TIPO: Eupompha fissiceps LeConte, 1858, por monotipia. Calospasta LeConte, 1862: $273(*)$

ESPECIE TIPO: Epicauta elegans LeConte, 1851, por monotipia.

COMENTARIOS TAXONÓMICOS: Selander (1954a) trató a Eupompha como sinónimo de Calospasta, pero Eupompha tiene prioridad, tal como el propio Selander (1955b) corrige posteriormente. Gemminger \& Harold (1870: 2147) deslizan el error tipográfico Calopasta, mientras que Van Dyke (1928: 401) incluye el nombre erróneo Colospasta.

CONTENIDO Y DISTRIBUCIÓN: El género cuenta con 12 especies exclusivas de la mitad septentrional de México (hasta Guerrero por el sur) y del suroeste de los Estados Unidos (Pinto, 1979; 1983; 1984c). El registro de Eupompha de Nicaragua (Maes, 1989) parece corresponder a un error de identificación (Maes \& Huether, 2007).

En México se encuentran E. elegans, E. decolorata, E. viridis, E. vizcaina, E. wenzeli, E. fissiceps, E. terminalis y E. sulcifrons (Pinto, 1984c). Además, E. imperialis (Wellman, 1912), E. schwarzi (Wellman, 1909) y E. histrionica (Horn, 1891) se han localizado en EEUU muy cerca de territorio mexicano.

\section{Eupompha decolorata (Horn, 1894)}

Calospasta decolorata Horn, 1894: 437 (*)

TERRA TYPICA: "Calimalli Mines, Baja California" tras designación de lectotipo. Lectotipo $n^{\circ} 155$, designado por Pinto (1979) en la colección de la California Academy of Sciences (San Francisco, EEUU).

Eupompha decolorata (Horn, 1894): Pinto, 1977: 944 (*)

COMENTARIOS TAXONÓMICOS: Pinto (1979) señala que los ejemplares de San Miguel Comondú podrían corresponder a una especie independiente.

DistribuCIÓN GEOGRÁFICA: Especie endémica de la Península de Baja California (Pinto, 1979). Se ha citado en los estados de Baja California y Baja California Sur.

MÉxico: Baja California: $25 \mathrm{mi}$. al oeste de la Bahía de los Ángeles (Pinto, 1983); Minas de Calimalli (Horn, 1894; Pinto, 1979); El Crucero (Pinto, 1983); 3 mi. al N de Punta Prieta (Pinto, 1979); 20 mi. al N de Punta Prieta (Pinto, 1979); 24 mi. al N de Punta Prieta (Pinto, 1979). Baja California Sur:
$39 \mathrm{~km}$ al oeste de San Ignacio (Pinto, 1983); 19 mi. al S de San Miguel Comondú (Pinto, 1979); $51 \mathrm{~km}$ al sureste de Guerrero Negro (Pinto, 1983); $25 \mathrm{~km}$ al este del Rancho San José Castro (Pinto, 1983); $39 \mathrm{~km}$ al oeste de San Ignacio (Pinto, 1983); 56 km al oeste de Vizcaíno (Pinto, 1983).

Eupompha elegans (LeConte, 1851)

Eupompha elegans elegans (LeConte, 1851)

Epicauta elegans LeConte, 1851: 161 (non Lytta elegans Klug, 1825) (*) TERRA TYPICA: "San Diego". Lectotipo no 5137, designado por Pinto (1979) en la colección del Museum of Comparative Zoology (Harvard University, Cambridge, EEUU

Lytta elegans (LeConte, 1851): LeConte, 1853: 341 (*)

Calospasta elegans (LeConte, 1851): LeConte, 1862: 273 (*)

Calospasta elegans var. humeralis Horn, 1870: 93 (*)

TERRA TYPICA: "Cariso Creek". Tipos posiblemente en la colección del Museum of Comparative Zoology de Harvard (EEUU).

Epicauta elegans (LeConte, 1851): Borchmann, 1917: 74 (*)

Eupompha elegans (LeConte, 1851): Gupta, 1965: 449 (*)

Eupompha elegans perpulchra (Horn, 1870)

Calospasta perpulchra Horn, 1870: 92 (*)

TERRA TYPICA: “Owen's Valley, and Posey Flat (S.E. Sierra region) Califormia". Lectotipo $\mathrm{n}^{\circ}$ 8082, designado por Pinto (1979) en la colección del Museum of Comparative Zoology (Harvard University, Cambridge, EEUU)

Colospasta elegans cyanea Van Dyke, 1929: $132(*)$

TERRA TYPICA: "Mt. Pinos, eastern Kern Co., Calif.". Holotipo n ${ }^{\circ}$ 2604, en la colección de la California Academy of Sciences (San Francisco, EEUU) (fide Van Dyke, 1929; Pinto, 1979)

Colospasta elegans perpulchra (Horn, 1870): Van Dyke, 1929: 132 (*)

Calospasta perpulchra var. mackenziei Papp, 1960: 76 (*)

TERRA TYPICA: "Mojave Desert, Victorville and Barstow".

Calospasta perpulchra var. bioculata Papp, 1960: 76 (*)

TERRA TYPICA: "Vincent area".

Eupompha elegans perpulchra (Horn, 1870): Pinto, 1979: 417 (*)

COMENTARIOS TAXONÓMICOS: Borchmann (1917) incluye a E. elegans simultáneamente en los géneros Epicauta y Calospasta. Beauregard (1890) considera a C. perpulchra como especie independiente. Van Dyke (1929) seguido por Doyen \& Opler (1973) consideran a E. perpulchra como subespecie de E. elegans. Pinto (1979) establece la sinonimia de E. e. cyanea, E. e. var. mackenziei y E. e. var. bioculata con E. e. perpulchra. Gemminger \& Harold (1870: 2147) deslizan el error tipográfico Calopasta elegans, mientras que Van Dyke (1928: 401) incluye el de Colospasta elegans.

DisTRIBUCIÓN GEOGRÁFICA: Especie distribuida por el noroeste de México y suroeste de los EEUU (Pinto, 1979 ; 1984a). La especie cuenta con dos subespecies, ambas presentes en Baja California, y una de ellas, E. $e$. perpulchra, también en Sonora.

Eupompha elegans elegans: MÉXICO: Baja California: Las Encinas, Sierra de San Pedro Mártir (Pinto, 1979); 3 mi. al E del Rancho Meling, Sierra de San Pedro Mártir (Pinto, 1979); San Felipe (Werner et al., 1966; Pinto, 1979); 2 mi. al O de Socorro, Sierra de San Pedro Mártir (Pinto, 1979); 5 mi. al E de Socorro, Sierra de San Pedro Mártir (Pinto, 1979).

Eupompha elegans perpulchra: MÉXICo: Baja California: San Felipe (Pinto, 1979). Sonora: Punta Rocosa (Rocky Point) (Pinto, 1979); Puerto Peñasco (Werner et al., 1966); Sonoyta 
(Werner et al., 1966); 30 mi. al S de Sonoyta (Pinto, 1979); 50 mi. al O de Sonoyta (Pinto, 1979); $27 \mathrm{mi}$. al E de San Luis (Pinto, 1979); 60 mi. al E de San Luis (Pinto, 1979).

Eupompha fissiceps LeConte, 1858

Eupompha fissiceps LeConte, 1858a: 21 (*)

TERRA TYPICA: “...probable on the Llano Estacado”. Holotipo en la colección del Museum of Comparative Zoology (Harvard University, Cambridge, EEUU) (fide Pinto, 1979). Cantharis fissiceps (LeConte, 1858): Beauregard, 1889: ccxii (*) Calospasta fissiceps (LeConte, 1858): Selander, 1954a: 12 (*)

DISTRIBUCIÓN GEOGRÁFICA: Especie distribuida por el norte de México y sur de los EEUU (Pinto, 1979; 1984a). En México se ha citado en los estados de Chihuahua, Coahuila y Durango (Champion, 1892; Pinto, 1979).

MÉXICo: Chihuahua: 42 mi. al SO de Camargo (Vaurie, 1950; Pinto, 1979); 110 mi. al S de Chihuahua (Pinto, 1979); 2 mi. al N de Sueco (Selander, 1954b). Coahuila: Guadalupe (Vaurie, 1950; Pinto, 1979); Saltillo (Werner et al., 1966; Pinto, 1979). Durango: Villa Lerdo (Dugès, 1889e; Champion, 1892; Vaurie, 1950; Werner et al., 1966; Pinto, 1979).

Eupompha sulcifrons (Champion, 1892)

Calospasta sulcifrons Champion, 1892: 394 (*)

Terra typica: "Mexico, Venta de Zopilote in Guerrero, 2800 feet". Lectotipo ${ }^{\circ} 155$, designado por Pinto (1979) en la colección del British Museum of Natural History (Londres). La serie típica consta de dos ejemplares más (Selander, 1954b).

Eupompha sulcifrons (Champion, 1892): Pinto, 1977: $944\left(^{*}\right.$

DisTRIBUCIÓN GEOGRÁFICA: Especie endémica de Guerrero (Pinto, 1979).

MÉxICo: Guerrero: 4 mi. al N de Chilpancingo (Pinto, 1979); 3 mi. al S de Iguala (Pinto, 1979); $30 \mathrm{~km}$ al S de Iguala (Pinto, 1979); 5 mi. al NNE de Mexcala (Selander, 1954b; Pinto, 1979); 1 mi. al S del Río Balsas en la carretera 95 cerca de Mexcala (Pinto, 1979); $3.5 \mathrm{mi}$. al S del Río Balsas en la carretera 95 (Pinto, 1979); Venta del Zopilote (Champion, 1892; Pinto, 1979); 9 km al N de Zumpango (Pinto, 1979).

Eupompha terminalis Selander, 1957

Eupompha (Eupompha) terminalis Selander, 1957a: 123 (*)

TERRA TYPICA: “Apatzingán, $1200 \mathrm{ft}$., Michoacán...”. Holotipo en la colección del US National Museum (fide Selander, 1957a; Pinto, 1979)

DisTRIBUCiÓN GEOGRÁFICA: Especie endémica de Michoacán (Pinto, 1979).

MÉXICo: Michoacán: Apatzingan (Pinto, 1979); 9 mi. al E de Capirio (Pinto, 1979).

\section{Eupompha viridis (Horn, 1883)}

Calospasta viridis Horn, 1883: 312 (*)

TERRA TYPICA: "Colorado and New Mexico" en la descripción original; "New Mexico" tras designación de lectotipo. Lectotipo $\mathrm{n}^{\mathrm{o}}$ 8088, designado por Pinto (1979) en la colección del Museum of Comparative Zoology (Harvard University, Cambridge, EEUU).

Eupompha viridis (Horn, 1883): Werner et al., 1966: $24\left({ }^{*}\right)$

DisTRIBUCIÓN GEOGRÁFICA: Especie distribuida por el norte de México y sur de los EEUU (Pinto, 1979). En México se ha citado en Chihuahua.
MÉXICo: Chihuahua: 30 mi. al S de Juárez (Pinto, 1979); 44 mi. al S de Juárez (Selander, 1954b; Werner et al., 1966; Pinto, 1979); Samalayuca (Pinto, 1979).

Eupompha vizcaina Pinto, 1983

Eupompha vizcaina Pinto, 1983: 184 (*)

TERRA TYPICA: "MEXICO. Baja California Sur, Vizcaino Peninsula, ca. $27^{\circ} 24^{\prime} \mathrm{N}, 114^{\circ} 05^{\prime} \mathrm{W} ; 51$ road km E Rancho San Jose Castro...'” Holotipo en la colección de la California Academy of Sciences (San Francisco, EEUU) (fide Pinto, 1983).

COMENTARIOS TAXONÓMICOS: Especie incluida en el grupo de E. viridis por Pinto (1983).

Distribución GEOGRÁficA: Especie endémica del Desierto Vizcaíno en la Península de Baja California (Pinto, 1983). Se ha citado en los estados de Baja California y Baja California Sur.

MÉxICo: Baja California: $9 \mathrm{~km}$ al norte de Guerrero Negro (Pinto, 1983); Miller's Landing [Atracadero de Miller] (Pinto, 1983). Baja California Sur: $51 \mathrm{~km}$ por carretera, al este del Rancho San José Castro, $27^{\circ} 24^{\prime} \mathrm{N}, 114^{\circ} 05^{\prime} \mathrm{W}$ (Pinto, 1983).

Eupompha wenzeli (Skinner, 1904)

Calospasta wenzeli Skinner, 1904: $217\left({ }^{*}\right)$

TERRA TYPICA: "Florence, Ariz.”. Serie típica en la colección de la Academy of Natural Sciences of Philadelphia (EEUU) (fide Skinner, 1904; Pinto, 1979).

Eupompha wenzeli (Skinner, 1904): Werner et al., 1966: 24 (*)

DisTRIBUCIÓN GEOGRÁFICA: Especie distribuida por el noroeste de México y suroeste de los EEUU (Pinto, 1979). En México se ha citado en Sonora.

MÉxICo: Sonora: Guaymas (Werner et al., 1966; Pinto, 1979); Llano (Pinto, 1979); San Francisco, carretera a La Salina (Pinto, 1979).

\section{Género Megetra LeConte, 1859}

Megetra LeConte, 1859: 127 (*)

ESPECIE TIPO: Meloe cancellatus Brandt et Erichson, 1832, por designación subsecuente de Wellman (1910b).

COMENTARIOS TAXONÓMICOS: Denier (1940: 425) desliza el error tipográfico Megatra, mientras que Zaragoza-Caballero (1999: 22) incluye el de Negetra.

CONTENIDO Y DISTRIBUCIÓN: El género cuenta con tres especies exclusivas de la mitad septentrional de México y del suroeste de los Estados Unidos (Pinto \& Bologna, 1999). Dos de ellas, M. cancellata y M. punctata se encuentran bien distribuidas en México, la tercera, M. vittata (LeConte, 1853), podría encontrarse en Coahuila.

Megetra cancellata (Brandt et Erichson, 1832)

Meloe cancellatus Brandt et Erichson, 1832: $141\left(^{*}\right)$

TERRA TYPICA: "Mexico". Tipos en la colección del Museum für Naturkunde Zentralinstitut der Humboldt-Universität de Berlin (fide Van Dyke, 1928; Selander, 1965a).

Cysteodemus cancellatus (Brandt et Erichson, 1832): LeConte, 1854d: $224(*)$

Megetra cancellata (Brandt et Erichson, 1832): LeConte, 1859: 127 (*) 
Megetra cancellata hoegei Dugès, 1889d: $39\left({ }^{*}\right)$

TERRA TYPICA: Tula, Hidalgo (fide Selander, 1965a). Tipo perdido según Selander (1965a). Vázquez \& Zaragoza (1979) indican como tipo el ejemplar $\mathrm{n}^{\circ} 2886$ (IBUNAM 022) de la colección del Instituto de Biología de la Universidad Nacional Autónoma de México.

Megetra cancellata championi Van Dyke, 1928: 456 (*)

TERRA TYPICA: "Villa Lerdo in Durango". Tipo en la colección del British Museum of Natural History (Londres) (fide Selander, 1965a).

Negetra cancellata var. hogei (Dugès, 1889): Zaragoza-Caballero, 1999: 22 (error tipográfico) $(*)$

COMENTARIOS TAXONÓMICOS: Selander (1965a) no considera válida la subespecie $M$. c. championi ya que, según indica, el patrón de variabilidad geográfica de esta especie es muy complicado. LeConte (1870) indica que las citas publicadas con anterioridad por él (LeConte, 1854d, 1859) no se refieren a esta especie. Denier (1935a: 176) menciona el nombre como Megetra (cancelalta) hoegei.

DISTRIBUCIÓN GEOGRÁFICA: Especie distribuida por el norte y centro de México y sur de los EEUU (Selander, 1965a; Pinto, 1984a). En México se ha citado en los estados de Aguascalientes, Chihuahua, Coahuila, Durango, Hidalgo, Nuevo León, San Luis Potosí y Zacatecas.

MÉXICo: Aguascalientes: $15 \mathrm{mi}$. al E de Aguascalientes (Selander, 1965a); El Retorno, $10 \mathrm{mi}$. al E de Aguascalientes (Selander, 1965a). Chihuahua: $42 \mathrm{mi}$. al SO de Ciudad Camargo (Vaurie, 1950 sub M. c. championi; Selander, 1965a); Salaices (Selander, 1965a); Santa Bárbara (Selander, 1965a). Coahuila: Cabos (Vaurie, 1950 sub M. c. championi; Selander, 1965a); Jaral (Selander, 1965a); $2 \mathrm{~km}$ al E de Ramos Arizpe (Selander, 1965a); Saltillo (Selander, 1965a). Durango: Villa [Ciudad] Lerdo (Dugès, 1889d; Champion, 1892; Vaurie, 1950 sub M. c. championi; Selander, 1965a); Diez de Octubre (San Lucas) (Vaurie, 1950 sub M. c. championi; Selander, 1965a); Donato Guerra (Selander, 1965a); Francisco I. Madero (Villa Madero) (Vaurie, 1950; Selander, 1965a); 1 mi. al SO de Villa Madero (Selander, 1965a); Guadalupe Victoria (Selander, 1965a); 7 mi. al NE de Guadalupe Victoria (Selander, 1965a); $3 \mathrm{mi}$. al ENE de Guadalupe Victoria (Selander, 1965a). Hidalgo: $18.5 \mathrm{mi}$. al O de Huichipán (Selander, 1965a); Pachuca (Champion, 1892; Vaurie, 1950; Selander, 1965a); Tula (Dugès, 1889d sub M. c. hoegei; Champion, 1892; Vaurie, 1950; Selander, 1965a; Vázquez \& Zaragoza, 1979 sub M. c. hoegei; Zaragoza-Caballero, 1999 sub N. c. hogei). Nuevo León: cerca de Galeana (Selander, 1965a); Mina (Selander, 1965a); carretera 57, Saltillo-Matehuala, $12 \mathrm{mi}$. al S del entronque con la carretera a San José de las Raíces (Selander, 1965a). San Luis Potosí: 12-50 mi. al NO de Ciudad del Maíz (Selander, 1965a); cerca de Huizache (Selander, 1965a); 36 km al N de Matehuala (Selander, 1965a); $25 \mathrm{~km}$ al E de Santo Domingo (Selander, 1965a); Arista (Villa de Arista) (Selander, 1965a). Zacatecas: 9 mi. al S de Fresnillo (Selander, 1965a); 13 mi. al NO de Zacatecas (Selander, 1965a).

\section{Megetra punctata Selander, 1965}

Megetra punctata Selander, 1965a: $563(*)$

TERRA TYPICA: "30 mi. S Chihuahua, $4000 \mathrm{ft}$., Chihuahua, México".

Holotipo en la colección del Chicago Natural History Museum (fide Selander, 1965a).
COMENTARIOS TAXONÓMICOS: Hasta su descripción formal, esta especie se ha venido confundiendo con $M$. vittata (Champion, 1892; Van Dyke, 1928; Blackwelder, 1945; Vaurie, 1950; MacSwain, 1956; Selander, 1954b).

DisTRIBUCIÓN GEOGRÁFICA: Especie distribuida por el norte de México y extremo sur de los EEUU (Selander, 1965a; Pinto, 1984a). En México se ha citado en los estados de Chihuahua, Coahuila, Durango y Sonora. Selander (1965a) indica que las citas mexicanas de $M$. vittata de Champion (1892), de MacSwain (1956) y también las de Selander (1954b), corresponden a M. punctata y por lo tanto también las referencias a México de Van Dyke (1928) y Blackwelder (1945), de forma que no existe ningún registro confirmado de la presencia de $M$. vittata en México.

MÉxICo: Chihuahua: $2.8 \mathrm{mi}$. al $\mathrm{S}$ de Moctezuma (MacSwain, 1956 sub M. vittata; Selander, 1965a); $42 \mathrm{mi}$. al SO de Ciudad Camargo (Vaurie, 1950 sub M. vittata; Selander, 1965a); Cañón de Bachimba, $27 \mathrm{mi}$. al S de Chihuahua (Selander, 1965a); 30-65 mi. al NO de Casas Grandes (Selander, 1965a); $80 \mathrm{mi}$. al NNO de Casas Grandes (Selander, 1965a); Catarinas (Vaurie, 1950 sub M. vittata; Selander, 1965a); Chihuahua (Champion, 1892 sub M. vittata; Van Dyke, 1928 sub $M$. vittata; Vaurie, 1950 sub M. vittata; Selander, 1965a); 7 mi. al N de Chihuahua (Selander, 1954b sub M. vittata; Selander, 1965a); $30 \mathrm{mi}$. al S de Chihuahua (Selander, 1954b sub M. vittata; Selander, 1965a); 27 mi. al SE de Chihuahua (Selander, 1965a); Gallego (Selander, 1965a); 2 mi. al S de Moctezuma (Selander, 1965a); Pinos Altos (Champion, 1892 sub M. vittata; Van Dyke, 1928 sub M. vittata; Vaurie, 1950 sub M. vittata; Selander, 1965a); Salaices (Selander, 1965a); Km. 36 de la carretera de Santa Bárbara a Ojito (Selander, 1965a); Santa Bárbara (Vaurie, $1950 \mathrm{sub} M$. vittata; Selander, 1965a); 2 mi. al S de Sueco (Selander, 1954b sub $M$. vittata; Selander, 1965a); $25 \mathrm{mi}$ al S de Sueco (Selander, 1965a); Valle de Olivos (Vaurie, 1950 sub M. vittata; Selander, 1965a). Coahuila: 25 mi. al SE de San Pedro de las Colonias (Vaurie, 1950 sub M. vittata; Selander, 1965a). Durango: Villa Lerdo (Champion, 1892 sub M. vittata; Van Dyke, 1928 sub M. vittata; Vaurie, 1950 sub M. vittata), Ciudad Lerdo (Selander, 1965a); 6 mi. al NE de El Salto (Vaurie, 1950 sub M. vittata; Selander, 1965a).

Género Phodaga LeConte, 1858

Phodaga LeConte, 1858b: 76 (*)

ESPECIE TIPO: Phodaga alticeps LeConte, 1858, por monotipia. Negalius Casey, 1891: 175 (*)

ESPECIE TIPO: Negalius marmoratus Casey, 1891, por monotipia.

COMENTARIOS TAXONÓMICOS: En la traducción al inglés del trabajo de Aksentjev (1989: 20) se desliza el error tipográfico Nogalius.

CONTENIDO Y DISTRIBUCión: El género cuenta con dos especies exclusivas del norte de México y del suroeste de los Estados Unidos (Pinto, 1972a,b; Pinto 1984a; Pinto \& Bologna, 1999). Pinto (1984a) establece la sinonimia de Negalius con Phodaga. 
Phodaga alticeps LeConte, 1858

Phodaga alticeps LeConte, 1858b: 76 (*)

TERRA TYPICA: "Sonora". Tipo en el Museum of Comparative

Zoology (Harvard University, Cambridge, EEUU)

DisTRIBUCIÓN GEOGRÁFICA: Especie distribuida por el noroeste de México y suroeste de los EEUU (Pinto, 1972a, 1984a). En México se ha citado en los estados de Baja California y Sonora.

MÉXICo: Baja California: San Felipe (Werner et al., 1966; Pinto, 1972a). Sonora: $10 \mathrm{mi}$. al Sur de Caborca (Pinto, 1972a); Puerto Peñasco (Werner et al., 1966; Pinto, 1972a); 20 mi. al Sureste de San Luis (Pinto, 1972a); Volcán Cerro Colorado, 35 mi. al Oeste de Sonoyta (Selander, 1958; Werner et al., 1966; Pinto, 1972a).

Phodaga marmorata (Casey, 1891)

Negalius marmoratus Casey, 1891: 175 (*)

TERRA TYPICA: "Western Texas". Dos sintipos en la colección del

United States National Museum of Natural History (Washington D.C.) (fide Pinto, 1984a).

Phodaga marmorata (Casey, 1891): Pinto (1984a): 135 (*)

DISTRIBUCIÓN GEOGRÁFICA: Especie distribuida por el norte de México y sur de los EEUU (Horn, 1896; Pinto, 1984a). En México se ha citado en los estados de Baja California Sur, Chihuahua y Sonora.

MÉxICo: Baja California Sur: $57 \mathrm{~km}$ al SE de Guerrero Negro (Pinto, 1984a); La Paz (Pinto, 1984a); $51 \mathrm{~km}$ al O de La Paz (Pinto, 1984a); 35 mi. al N de Loreto (Pinto, 1984a); 48 $\mathrm{km}$ al S de Loreto (Pinto, 1984a); El Coyote, $26 \mathrm{~km}$ al SSE de Mulegé (Pinto, 1984a); Pescadero (Pinto, 1984a); 7 mi. al S de San Antonio (Pinto, 1984a); San José del Cabo (Horn, 1896); $9 \mathrm{~km}$ al SE de Santa Rita (Pinto, 1984a). Chihuahua: 5 mi. al $\mathrm{N}$ de Ojo del Lucero (Pinto, 1984a); $29 \mathrm{~km}$ al N de Samalayuca (Pinto, 1984a). Sonora: Empalme (Pinto, 1984a); Guaymas (Werner et al., 1966); $51 \mathrm{~km}$ al $\mathrm{N}$ de Guaymas (Pinto, 1984a); 3 mi. al N de Santa Ana (Pinto, 1984a).

Género Pleuropasta Wellman, 1909

Pleuropasta Wellman, 1909: 20 (*)

ESPECIE TIPO: Calospasta mirabilis Horn, 1870, por designación original.

Pleurospasta Wellman, 1909: Van Dyke, 1928: 401 (enmienda injustificada) (*)

CONTENIDO Y DISTRIBUCIÓN: El género cuenta con dos especies exclusivas de la mitad septentrional de México y del suroeste de los Estados Unidos (Pinto \& Bologna, 1999). Ambas especies, $P$. mirabilis y $P$. reticulata están presentes en México (Pinto, 1984c).

Pleuropasta mirabilis (Horn, 1870)

Calospasta mirabilis Horn, 1870: 93 (*)

TERRA TYPICA: "Southern Arizona....". Holotipo en la colección del

Museum of Comparative Zoology (Harvard University, Cambridge, EEUU) (fide Pinto, 1984a).

Pleuropasta mirabilis (Horn, 1870): Wellman, 1909: 21 (*)

Pleurospasta mirabilis (Horn, 1870): Van Dyke, 1947: 157 (*)

COMENTARIOS TAXONÓMICOS: Van Dyke (1947) trató a $P$. mirabilis y $P$. reticulata como subespecies de una única especie.
DISTRIBUCIÓN GEOGRÁFICA: Especie distribuida por el norte de México y suroeste de los EEUU (Pinto, 1984a). En México se ha citado en los estados de Baja California, Baja California Sur y Sonora. Pinto (1984a) señala que la cita mexicana de Champion (1892), como ya indicó Vaurie (1950), y algunas de las de Dillon (1952) de $P$. mirabilis corresponden a P. reticulata.

MÉxICo: Baja California: $1 \mathrm{mi}$. al E del Rancho Meling (Pinto, 1984a); $9 \mathrm{~km}$ al NO del Rancho Inés $\left(29^{\circ} 46^{\prime} \mathrm{N}, 114^{\circ}\right.$ 46' O) (Pinto, 1984a); San Felipe (Werner et al., 1966; Pinto, 1984a); 14 mi. al S de San Felipe (Pinto, 1984a); San Julio (Horn, 1894). Baja California Sur: $19 \mathrm{~km}$ al NO de Mulegé (Pinto, 1984a); $39 \mathrm{~km}$ al O de San Ignacio (Pinto, 1984a). Sonora: 36 mi. al O de El Golfo (Pinto, 1984a).

Pleuropasta reticulata Van Dyke, 1947

Pleurospasta mirabilis reticulata Van Dyke, 1947: 158 (y error en el género) (*)

TeRra tYPICA: "Loving, New MeXico...". Holotipo, nº5870, en la colección de la California Academy of Sciences (San Francisco, EEUU) (fide Van Dyke, 1947; Pinto, 1984a).

Pleuropasta reticulata Van Dyke, 1947: Cohen et Pinto, 1977: 741 (*)

COMENTARIOS TAXONÓMICOS: Vaurie (1950), seguida por Cohen \& Pinto (1977), propone estatus específico para las dos subespecies de Van Dyke (1947).

DISTRIBUCIÓN GEOGRÁFICA: Especie distribuida por el norte de México y sur de los EEUU (Pinto, 1984a). En México se ha citado en los estados de Chihuahua, Coahuila y Durango. Pinto (1984a) señala que la cita mexicana de Champion (1892) (Coahuila sin más precisión) y algunas de las de Dillon (1952) de P. mirabilis corresponden a $P$. reticulata.

MÉxICo: Chihuahua: 33 mi. al N de Hidalgo del Parral (Pinto, 1984a). Coahuila: Cuesta La Muralla, en la carretera 57 (Pinto, 1984a). Nuevo León: $8 \mathrm{~km}$ al SE de Villa de García (Pinto, 1984a). Tamaulipas: Nuevo Laredo (Van Dyke, 1947; Vaurie, 1950; Pinto, 1984a)

\section{Género Tegrodera LeConte, 1851}

Tegrodera LeConte, 1851: 159 (*)

ESPECIE TIPO: Tegrodera erosa LeConte, 1851, por monotipia.

CONTENIDO Y DISTRIBUCIÓN: El género cuenta con tres especies exclusivas del noroeste de México y del suroeste de los Estados Unidos (Pinto, 1975a,c; Pinto \& Bologna, 1999). Dos de ellas, T. erosa y T. aloga se encuentran en México (Pinto, 1984c).

Tegrodera aloga Skinner, 1903

Tegrodera aloga Skinner, 1903: 168 (*)

TERRA TYPICA: "the banks of the Gila River, near Florence Arizona". Lectotipo designado por Pinto (1975a) (tipo no 8198) en la colección de la Academy of Natural Sciences of Philadelphia (EEUU).

COMENTARIOS TAXONÓMICOS: Descrita ambiguamente como especie (Skinner, 1903), ya fue tratada a nivel específico por diversos autores, aunque su estatus actual no se consolidó hasta el trabajo de Pinto (1975a). 
DISTRIBUCIÓN GEOGRÁFICA: Especie distribuida por el norte de México y sur de los EEUU (Pinto, 1984a). En México se ha citado en Sonora.

MÉxICo: Sonora: Caborca (Pinto, 1975a); $10 \mathrm{mi}$. al Este del Dátil (Pinto, 1975a); Guaymas (Pinto, 1975a); 30 mi. al Sur de Hermosillo (Pinto, 1975a); 3.5 mi. al Norte de Hermosillo (Pinto, 1975a); 40 mi. al Norte de Hermosillo (Pinto, 1975a); Volcán Cerro Colorado, 35 mi. al Oeste de Sonoyta (Selander, 1958 sub T. erosa; Werner et al., 1966); $43 \mathrm{~km}$ al Sur de Sonoita (Pinto, 1975a); Tubatama (Pinto, 1975a).

Tegrodera erosa LeConte, 1851

Tegrodera erosa erosa LeConte, 1851

Tegrodera erosa LeConte, 1851: 159 (*)

TERRA TYPICA: "San Diego". Lectotipo (tipo $\mathrm{n}^{\circ}$ 5138) designado por Pinto (1975a) en la colección del Museum of Comparative Zoology de la Universidad de Harvard (EEUU).

Lytta erosa (LeConte, 1851): LeConte, 1853: $342(*)$

Cantharis erosa (LeConte, 1851): Beauregard, 1890: 493 (*)

Tegrodera erosa extincta Beauregard, 1890

Cantharis erosa var. extincta Beauregard, 1890: 493 (*)

TERRA TYPICA: no indicada. Los dos ejemplares de la serie típica de la Colección Beauregard (fide Beauregard, 1890) están en paredero desconocido (fide Pinto, 1975a).

Tegrodera erosa var. inornata Blaisdell, 1918: 334 (*)

TERRA TYPICA: “Arizona”, posiblemente errónea (Pinto, 1975a).

Holotipo en la colección de la California Academy of Sciences de

San Francisco (Estados Unidos) (fide Blaisdell, 1918; Pinto, 1975a).

Tegrodera erosa extincta (Beauregard, 1890): Denier, 1935a: 176 (*)

COMENTARIos TAXonÓmicos: Pinto (1975a) establece el estatus subespecífico de $T$. e. inornata y trata a $T$. $e$. extincta como un nomen dubium. Sin embargo la descripción de Beauregard (1890) aunque breve, responde totalmente a los caracteres de esta subespecie y por lo tanto no parece justificado ignorar su existencia. Por lo tanto, seguimos a Denier (1935a) y recuperamos el nombre de Beauregard (1890) para esta raza. Sea cual sea el nombre considerado en la actualidad, se reconoce la existencia de dos subespecies bien diferenciadas dentro de T. erosa.

DISTRIBUCIÓN GEOGRÁFICA: Especie distribuida por el noroeste de México y suroeste de los EEUU (Pinto, 1975a, 1984a). En México se ha citado en Baja California, Baja California Sur y Sinaloa. Las dos subespecies están presentes en México.

Tegrodera erosa erosa: MÉXICo: Baja California: Ensenada (Pinto, 1975a); 5 mi. al Este de El Rosario (Pinto, 1975a); 10 mi. al noroeste del Rancho Hamilton (Pinto, 1975a); Ojos Negros (Pinto, 1975a); Sangre de Cristo (Pinto, 1975a); “Tia Juana” [Tijuana] (Blaisdell, 1918).

Tegrodera erosa extincta: MÉXICo: Baja California: Cañón del Cabañe (sic), Sierra de San Pedro Mártir (Pinto, 1975a); Cañón del Parral, Sierra de San Pedro Mártir (Pinto,

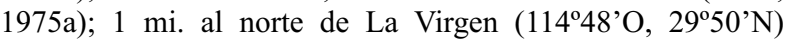
(Pinto, 1975a); Rancho Meling, Sierra de San Pedro Mártir (Pinto, 1975a); 12 y 13 mi. al norte de Puertecitos (Pinto, 1975a); 40 mi. al sureste de Puertecitos (Pinto, 1975a); Rancho Mesquital (Pinto, 1975a); 4, 7, 20 y $66 \mathrm{mi}$. al norte de San Felipe (Pinto, 1975a); 30 y 32 mi. al sur de San Felipe (Pinto, 1975a); Mina de Azufre al sur de San Felipe (Pinto, 1975a); 27 mi. al sur de San Vicente (Pinto, 1975a); Valle de Trinidad (Pinto, 1975a). Baja California Sur: Bahía de La Concepción (Pinto, 1975a); 7 mi. al oeste de la Bahía de San Francisquito (Pinto, 1975a); $30 \mathrm{mi}$. al norte de Loreto (Pinto, 1975a); $70 \mathrm{~km}$ al sureste de Guerrero Negro (Pinto, 1975a); San José del Cabo (Pinto, 1975a); San José de Gracia (Horn, 1894); $18 \mathrm{mi}$. al suroeste de San Miguel Comondú (Pinto, 1975a). Sinaloa: 5.4 mi. al norte de Guamuchil (Pinto, 1975a).

Tribu Lyttini LeConte, 1862

Lyttae LeConte, 1862: 269 (*)

GÉNERO TIPO: Lytta Fabricius, 1775.

CONTENIDO Y DISTRIBUCIÓN: La tribu tiene amplia dispersión mundial. Está representada en América por 6 géneros: Acrolytta Kaszab, 1959, Dictyolytta Selander, 1960, Lytta, Lyttana Pinto \& Bologna, 1997, Megalytta Selander, 1960 y Parameloe Denier, 1933, de los cuales únicamente Lytta está presente en México (Pinto \& Bologna, 1999; Bologna \& Pinto, 2007). De acuerdo con estos autores, la sistemática de los géneros de América del Sur requiere una revisión profunda.

Género Lytta Fabricius, 1775

Lytta Fabricius, 1775: 260 (*)

ESPECIE TIPO: Meloe vesicatoria Linnaeus, 1758, por designación subsecuente de Wellman (1910b).

CONTENIDO Y DISTRIBUCIÓN: El género cuenta con 109 especies repartidas en 9 subgéneros que se distribuyen por la región Holártica, aunque su presencia en América del Sur es un tema ampliamente debatido (Pinto \& Bologna, 1999). En América del Norte se conocen 69 especies distribuidas en 4 subgéneros (Adicolytta, Paralytta, Pomphopoea LeConte, 1862 y Poreospasta), extendidas desde el centro del Canadá hasta Panamá (Pinto \& Bologna, 1999). Las especies conocidas en México se agrupan en 3 subgéneros, Adicolytta, Paralytta y Poreospasta. Blackwelder (1945) señaló a Lytta puberula LeConte, 1866 y Lytta bruchi Pic, 1927 como especies presentes en la fauna mexicana, pero según Selander (1960a) ninguna de las dos se encuentra en México.

En obras antiguas (Geoffroy, 1762; Lacordaire, 1859; Horn, 1873; Champion, 1892; Fall, 1901a,b) se ha utilizado incorrectamente el nombre de Cantharis Linnaeus, 1758, para referirse al género Lytta. El problema se originó cuando Linneo utilizó como nombre científico el nombre vernáculo "cantárida" con el que se conocía a ciertos meloidos (entre ellos Lytta vesicatoria) y lo aplicó a otros coleópteros que ni poseen cantaridina ni están relacionados con los meloidos (los actuales Cantharidae). Geoffroy (1762) trató de corregir el error, pero las reglas nomenclaturales no permiten tal acción.

Subgénero Adicolytta Selander, 1960

Lytta (Adicolytta) Selander, 1960a: 137 (*)

ESPECIE TIPO: Cantharis mutilata Horn, 1875, por designación original. 
CONTENIDO Y DISTRIBUCIÓN: El subgénero incluye 7 especies, todas exclusivas de América del Norte, desde el sur de EEUU hasta Panamá (Selander, 1960a). Todas las especies del subgénero se encuentra en México y de las 7 especies, 5 son endémicas.

Selander (1960a) divide el subgénero en 3 grupos de especies: los grupos de L. peninsularis, L. eucera y $L$. mutilata.

Lytta (Adicolytta) cardinalis Chevrolat, 1833

Lytta cardinalis Chevrolat, 1833: fasc. $1, \mathrm{n}^{\circ} 13$ (sin paginar) (*)

TeRra tyPICA: "Tehuacan". Tipos procedentes del museo D. de

Romand (Chevrolat, 1833) sin localización actual conocida (fide

Selander, 1960a).

Cantharis cardinalis (Chevrolat, 1833): Dugès, 1869a: $111(*)$

Comentarios taxonómicos: LeConte (1858a), Beauregard (1890) y Borchmann (1917) incluyeron a $L$. fulvipennis en la sinonimia de L. cardinalis, aunque Selander (1960a) no comparte su criterio. Beauregard (1890) y Champion (1892) incluyen en la sinonimia dos nombres no disponibles: L. dejeani Höpfner in Dejean, 1837: 246 y L. sanguinipennis Klug in Dejean, 1837: 246, y Sturm, 1843: 174.

DisTRIBUCiÓN GEOGRÁFICA: Especie endémica de México (Selander, 1960a), aunque Pinto \& Bologna (1999) citan la existencia de dos ejemplares con etiqueta de "Peru. La Merced" que posiblemente correspondan a un error de etiquetado.

MÉxICo: Guerrero: Mochitlán (Champion, 1892; Selander, 1960a). Jalisco: Guadalajara (Selander, 1960a). Morelos: Alpuyeca (Selander, 1960a); Cuautla (Selander, 1960a); Xochicalco (Selander, 1960a); Yautepec (Selander, 1960a). Oaxaca: Huajuapan de León (Selander, 1960a); Tamazulapan (Selander, 1960a). Puebla: Chiautla (Selander, 1960a); Izúcar de Matamoros (Champion, 1882; Selander, 1960a); Puebla (Champion, 1882; Selander, 1960a); Techamachalco (Selander, 1960a); Tehuacán (Chevrolat, 1833; Champion, 1882; Selander, 1960a). Veracruz: Veracruz (Dugès, 1889e).

Lytta (Adicolytta) erebea (Champion, 1892)

Cantharis erebea Champion, 1892: 442 (*)

TERRA TYPICA: “MeXICO..., Huetamo in Michoacan...”. La serie típica consta de un macho y 3 hembras, está en el British Museum of Natural History (Londres) (fide Selander, 1960a)

Lytta erebea (Champion, 1892): Borchmann, 1917: 93 (*)

COMENTARIOS TAXONÓMICOS: Transferida al subgénero Adicolytta por Selander (1960a).

DisTRIBUCIÓN GEOGRÁFICA: Endemismo mexicano citado en Michoacán y México.

MÉxICo: México: Temescaltepec (Selander, 1960a). Michoacán: Huetamo (Champion, 1892; Selander, 1960a).

Lytta (Adicolytta) erythrothorax (Mendoza et Herrera, 1867)

Cantharis erytrotora Mendoza et Herrera, in Herrera et Mendoza, 1867: $14(*)$
TERRA TYPICA: "Distrito de Tasco" originalmente; "Amula, Guerrero" tras designación de neotipo (Selander, 1960a). Tipo orinal perdido, neotipo macho de "Amula, Guerrero" de la colección del British Museum of Natural History (Londres) (Selander, 1960a).

Cantharis erythrotorax Herrera et Mendoza, 1867: Dugès, 1870: 166 (enmienda injustificada) ${ }^{*}$ )

Cantharis erythrothorax Herrera et Mendoza, 1867: Dugès, 1886a: 582 (enmienda justificada) (*)

COMENTARIOS TAXONÓMICOS: Selander (1960a) trata el error en la figura de Dugès (1881: lám. 4, fig. 10), que queriendo figurar su $C$. stigmata incluye en realidad un dibujo de Lytta erythrotorax, como la descripción de un taxon nuevo, homónimo de C. stigmata Dugès, 1869, y llega a designar como neotipo un ejemplar con etiqueta "México (Dugès collection)" del United States National Museum (Washington D.C.). Toda esta acción taxonómica es innecasaria ya que se trata de un mero error. Dugès (1874b), Beauregard (1890) y Champion (1892, 1899 ) incluyen en la sinonimia el nombre no disponible L. bisignata Sturm, 1843: 174. La enmienda propuesta por Dugès (1886a) estaría justificada según el artículo 33.3.1 del Código Internacional de Nomenclatura Zoológica, CINZ, 2000). Denier (1935a: 163) incluye el error tipográfico Lytta erythothorax.

DisTRIBUCIÓN GEOGRÁFICA: Endemismo mexicano, aunque existe un ejemplar en el United States National Museum con una etiqueta equivocada de Texas (Selander, 1960a).

MÉxICo: Colima: Colima (Selander, 1960a); Tonila (Champion, 1882; Selander, 1960a). Guanajuato: Tupátaro (Dugès, 1889e; Champion, 1882; Selander, 1960a); 6 mi. al NO de Yuriria (Selander, 1960a). Guerrero: Amula (Champion, 1882; Selander, 1960a); Michapa (posiblemente corresponda a la localidad de Oaxaca: Los Reyes Michapa, fide Selander, 1960a) (Selander, 1960a); Taxco de Alarcón (Dugès, 1870; Champion, 1882; Selander, 1960a). Jalisco: Guadalajara (Selander, 1960a); Volcán de Colima (Selander, 1960a). Michoacán: Morelia (Selander, 1960a). Morelos: Cuernavaca (Champion, 1882; Selander, 1960a); Jalastoc (Jaloxtoc) (Selander, 1960a); Tepoztlán (Selander, 1960a). Nayarit: alrededores de Compostela (Selander, 1960a); San Blas (Selander, 1960a). Sinaloa: Mazatlán (Selander, 1960a); Pericos (Selander, 1960a); Venodio (posiblemente Venadillo, fide Selander, 1960a) (Selander, 1960a). Sonora: San Bernardo (Selander, 1960a). Veracruz: Veracruz (Dugès, 1889e).

Lytta (Adicolytta) eucera (Chevrolat, 1834)

Cantharis eucera Chevrolat, 1834a: fasc. $3, \mathrm{n}^{\circ} 56$ (sin paginar) (*)

Terra typica: "Guatimala (sic), Mexico". Localización del tipo desconocida (fide Selander, 1960a).

Cantharis spectabilis Laporte de Castelnau, 1840: 273

TERRA TYPICA: México (fide Selander, 1960a). Localización del tipo desconocida (fide Selander, 1960a).

Lytta eucera (Chevrolat, 1834): Borchmann, 1917: 94 (*)

COMENTARIOS TAXONÓMICOS: Beauregard (1890), seguido por Champion (1892), incluye en la sinonimia de L. eucera a $C$. spectabilis, donde ha permanecido desde 
entonces, y también menciona el nombre no disponible $C$. occipitalis Sturm. Chevrolat (1834a) menciona en la sinonimia el nombre no disponible Lytta eucera Klug. Incluida en el subgénero Adicolytta por Selander (1960a). Horn (1883: 310) incluye el error tipográfico Cantharis encera.

DISTRIBUCIÓN GEOGRÁFICA: Especie ampliamente extendida desde el norte de México hasta Panamá, incluyendo citas en Guatemala y Costa Rica (Selander, 1960a). Las citas de Texas (Horn, 1873; Fall, 1901b; Dillon, 1952) son dudosas (Selander, 1960a).

MÉxICo: Chiapas: Finca La Isla (Selander, 1960a). Coahuila: Monclova (Selander, 1960a). Colima: Zapotlán (Champion, 1892; Selander, 1960a). Distrito Federal: alrededores de México (Herrera \& Mendoza, 1866); Ciudad Madero (Selander, 1960a); Ciudad de México (Dugès, 1869a; Dugès, 1889e; Champion, 1892; Selander, 1960a); Coapa (Selander, 1960a); San Ángel (Selander, 1960a); San "P." de los Pinos (Selander, 1960a); Tacuba (Selander, 1960a); Tacubaya (Selander, 1960a); Valle de México (Peñafiel Barranco, 1866; Selander, 1960a). Durango: Sierra de Durango (Selander, 1960a). Guanajuato: Guanajuato (Champion, 1892); Silao (Dugès, 1869a; Champion, 1892; Selander, 1960a). Hidalgo: Atotonilco el Grande (Peñafiel Barranco, 1866; Dugès, 1869a; Champion, 1892; Selander, 1960a); Pachuca (Peñafiel Barranco, 1866; Dugès, 1869a; Champion, 1892; Selander, 1960a). Jalisco: Tlaquepaque (Selander, 1960a). México: Chapingo (Selander, 1960a); Jilotepec (Herrera \& Mendoza, 1866; Selander, 1960a); San Rafael (Selander, 1960a); Temescaltepec (Selander, 1960a); Texcoco (Selander, 1960a). Michoacán: Morelia (Dugès, 1889e; Champion, 1892; Selander, 1960a); Tacámbaro (de Codallos) (Champion, 1892; Selander, 1960a); Tancítaro (Selander, 1960a). Morelos: Cuernavaca (Champion, 1892; Selander, 1960a); Puente de Ixtla (Selander, 1960a); Tepoztlán (Selander, 1960a). Oaxaca: La Parada (Champion, 1892; Selander, 1960a); Oaxaca de Juárez (Champion, 1892; Selander, 1960a). Puebla: Atlixco (Selander, 1960a); Cholula (Champion, 1892; Selander, 1960a); La Esperanza (Champion, 1892; Selander, 1960a); Huauchinango (Herrera \& Mendoza, 1866); Izúcar de Matamoros (Selander, 1960a); Puebla (Champion, 1892; Selander, 1960a); Techamachalco (Selander, 1960a). San Luis Potosí: Ciudad Valles (Selander, 1960a); Huasteca Potosina (Dugès, 1889e; Champion, 1892; Selander, 1960a). Veracruz: Xalapa (Champion, 1892; Selander, 1960a); Orizaba (Champion, 1892; Selander, 1960a). Sin localizar: Finca Gibralter (Selander, 1960a); Tierra Caliente (Dugès, 1889e).

\section{Lytta (Adicolytta) mutilata (Horn, 1875)}

\section{Cantharis (Lytta) mutilata Horn, 1875: 155 (*)}

TERRA TYPICA: "Arizona". El tipo posiblemente está en la colección del Museum of Comparative Zoology de Harvard (fide Selander, 1960a).

Lytta mutilata (Horn, 1875): Borchmann, 1917: 96 (*)

COMENTARIOS TAXONÓMICOS: Incluida en el subgénero Adicolytta por Selander (1960a).

DISTRIBUCIÓN GEOGRÁFICA: Distribuida en zonas áridas del norte de México y suroeste de los Estados Unidos (Selander, 1960a). Citada en Baja California, Chihuahua, Coahuila y Durango.
MÉXICo: Baja California: San Julio (Horn, 1894; Selander, 1960a). Chihuahua: $9 \mathrm{mi}$. al N de Camargo (Selander, 1960a); Salaices (Selander, 1960a); Km 36 entre Santa Bárbara y Ojito (Durango) (Selander, 1960a). Coahuila: Monclova (Champion, 1892; Vaurie, 1950; Selander, 1960a). Durango: Ciudad Lerdo (Champion, 1892; Vaurie, 1950; Selander, 1960a).

Lytta (Adicolytta) peninsularis (Fall, 1901)

Cantharis peninsularis Fall, 1901b: 300 (*)

TERRA TYPICA: "Cape San Lucas, Lower California". El tipo se encuentra en el Museum of Comparative Zoology (Harvard University, Cambridge, EEUU) (fide Selander, 1960a).

Lytta peninsularis (Fall, 1901): Borchmann, 1917: 97 (*)

COMENTARIOS TAXONÓMICOS: Incluida en el subgénero Adicolytta por Selander (1960a). Denier (1935a: 164) publica el error tipográfico Lytta penincularis.

DisTRIBUCIÓN GEOGRÁfiCA: Sólo conocida de la localidad típica.

MÉxICo: Baja California Sur: Cabo San Lucas (Fall, 1901b; Selander, 1960a).

Lytta (Adicolytta) sanguinea Haag-Rutenberg, 1880

Lytta sanguinea Haag-Rutenberg, 1880: 35 (*)

TERRA TYPICA: "Mexico". Holotipo en la colección del Zoologische Staatsammlung de Munich (fide Selander, 1960a)

Cantharis sanguinea (Haag-Rutenberg, 1880): Beauregard, 1890: 496 (*)

COMENTARIOS TAXONÓMICOS: Incluida en el subgénero Adicolytta por Selander (1960a).

DistribuCión GEOGRÁfICA: Endemismo mexicano citado en Guerrero, Michoacán y Morelos.

MÉXICO: Guerrero: Iguala (Selander, 1960a); Mochitlán (Champion, 1892; Selander, 1960a); Río Balsas (Selander, 1960a). Michoacán: Apatzingan de la Constitución (Selander, 1960a). Morelos: 16 mi. al S de Cuernavaca (Selander, 1960a); Jojutla (Selander, 1960a).

\section{Subgénero Paralytta Selander, 1960}

Lytta (Paralytta) Selander, 1960a: 49 (*)

ESPECIE TIPO: Lytta magister Horn, 1870, por designación original

CONTENIDO Y DISTRIBUCIÓN: El subgénero incluye 37 especies, todas exclusivas de América del Norte, desde el sur de México hasta British Columbia en el Canadá (Selander, 1960a; Pinto, 1985). De las 25 especies registradas en México, 18 son endémicas.

Además de estas 25 especies, podrían encontrarse en México, ya que se han citado en los EEUU, muy cerca de la frontera: Lytta (Paralytta) fulvipennis LeConte, 1853, especie ampliamente extendida por el sureste de los EEUU, con numerosas citas en Texas (Dillon, 1952; Selander, 1960a); Lytta (Paralytta) reticulata Say, 1824, ampliamente extendida por la región centro-meridional de los EEUU, con citas en la frontera de Texas (Selander, 1960a); Lytta (Paralytta) mirifica Werner, 1951, especie descrita y únicamente conocida de Anthony, en el Valle del Río Grande (Nuevo México, EEUU), muy cerca de 
México (Selander, 1960a); Lytta (Paralytta) tenebrosa (LeConte, 1851), extendida por el centro y sur de California (EEUU), con citas en San Diego (Selander, 1960a) y Lytta (Paralytta) morosa (Fall, 1901b), especie exclusiva del extremo sur de California (EEUU), con citas en San Diego (Selander, 1960a).

Selander (1960a) divide el subgénero en 6 grupos de especies: los grupos de L. nitidicollis, L. fulvipennis, $L$. magister, L. variabilis y L. reticulata, aunque señala que los dos útltimos podrían reunirse en uno sólo. Pinto (1985) indica que las diferencias entre algunos de estos grupos están muy poco marcadas y que existen especies con caracteres pertenecientes a más de un grupo, por lo que el subgénero necesitaría una nueva revisión.

Lytta (Paralytta) augusti Haag-Rutenberg, 1880

Lytta variabilis var. augusti Haag-Rutenberg, 1880: 38 (nota al pie de página) (*)

TERRA TYPICA: "Mexico". La serie típica de L. augusti incluye un macho y dos hembras (parte de la serie de variabilis) con etiqueta "Mexico, Deyrolle". De éstos, Selander (1960a) designa el macho como lectotipo en la colección del Zoologische Staatssammlung de Munich.

Cantharis subviolacea Champion, 1892: 445 (*)

Terra typica: "MeXico, Parada, Duraznal...”. El tipo está en el British Museum of Natural History (Londres) (fide Selander, 1960a)

COMENTARIOS TAXONÓMICOS: Descrita originalmente como variedad de L. variabilis, Selander (1954) la eleva a la categoría de especie. Incluida en el subgénero Paralytta por Selander (1960a). Beauregard (1890) indica, como sinónimo de $C$. puberula, el nombre no disponible Cantharis puberula var. augusti Deyrolle, mientras que Champion (1892) recoge de nuevo en la sinonimia de $C$. subviolacea, el nombre no disponible Cantharis augusti Deyr.

DisTRIBUCIÓN GEOGRÁFICA: Endemismo mexicano, citado en los estados de Oaxaca y Veracruz.

MÉXICo: Oaxaca: Duraznal (Champion, 1892 sub C. subviolacea; Selander, 1960a); La Parada (Champion, 1892 sub C. subviolacea; Selander, 1960a); Oaxaca de Juárez (Selander, 1954b; Selander, 1960a). Veracruz: El Puerto, 31 mi. al SO de Orizaba (Selander, 1954b; Selander, 1960a).

Lytta (Paralytta) biguttata LeConte, 1853

Lytta (Paralytta) biguttata biguttata LeConte, 1853

Lytta biguttata LeConte, 1853: 332 (*)

TerRa TYPICA: "Santa Fe, New Mexico". Holotipo en el Museum of Comparative Zoology (Harvard University, Cambridge, EEUU) (fide Selander, 1960a).

Cantharis biguttata (LeConte, 1853): Horn, 1873: $109(*)$

Cantharis alemani Dugès, 1889e: $106(*)$

TERRA TYPICA: "Villa Lerdo". Aparentemente, el tipo está perdido (fide Selander, 1960a).

Lytta (Paralytta) biguttata bivirgata (Dugès, 1881)

Cantharis bivirgata A. Dugès in E. Dugès, 1881: $140(*)$

TERRA TYPICA: "cerca de Silao", originalmente; "Guanajuato", tras designación un ejemplar hembra como neotipo (Selander, 1960a) en la colección del Zoologische Staatssammlung de Munich.
Cantharis alfredi Dugès, 1889e: 98 (*)

TERRA TYPICA: "Silao y Tupátaro".

Lytta biguttata bivirgata (Dugès, 1881): Selander, 1956: 125 (*)

Lytta (Paralytta) biguttata pallens Selander, 1956

Lytta biguttata pallens Selander, 1956: 119 (*)

TERRA TYPICA: "Douglas, Arizona". Tipo en la colección del Essig Museum of Entomology (University of California, Berkeley, EEUU) (fide Selander, 1960a)

COMENTARIOS TAXONÓMICOS: Dugès (1886a: 582) ya utilizó el nombre Cantharis alfredi sin acompañarlo de una decripción. Posteriormente describió $C$. alfredi al considerar erróneamente que $C$. bivirgata era un nombre "in litteris" (Dugès, 1889e). Selander (1956) establece la sinonimia de $C$. alemani con $C$. b. biguttata. Cantharis alemani había sido considerado por Borchmann (1917) como una variedad de L. quadrimaculata. Selander (1956) trata a C. bivirgata como subespecie de Lytta biguttata, aunque posteriormente (Selander, 1960) únicamente considera a estos grupos de poblaciones como razas biológicas sin estatus formal. En este catálogo consideramos la existencia de tres subespecies.

DISTRIBUCIÓN GEOGRÁFICA: Ampliamente distribuida en México y en el centro y sur de EEUU (Selander, 1960a). En México se ha citado en los estados de Chihuahua, Coahuila, Durango, Guanajuato, México y San Luis Potosí.

L. biguttata biguttata: MÉXICo: Coahuila: Cabos (Vaurie, 1950; Selander, 1960a); Guadalupe (Vaurie, 1950; Selander, 1960a); La Gloria, al S de Monclova (Vaurie, 1950; Selander, 1960a); La Rosa (Vaurie, 1950; Selander, 1960a); Paila (Vaurie, 1950; Selander, 1960a); 5 mi. al N de Saltillo (Vaurie, 1950; Selander, 1960a); San Pedro de Colonias (Vaurie, 1950; Selander, 1960a); $25 \mathrm{mi}$. al SE de San Pedro de Colonias (Vaurie, 1950; Selander, 1960a); Torreón (Selander, 1960a). Durango: Ciudad Lerdo (Dugès, 1889e sub C. alemani; Champion, 1892; Vaurie, 1950; Selander, 1956, 1960a); San Lucas (Vaurie, 1950; Selander, 1960a); Yerbanís, Distrito de Cuencamé (Vaurie, 1950; Selander, 1960a). San Luis Potosí: El Huizache (Selander, 1960a).

L. biguttata pallens: MÉXICO: Chihuahua: $20 \mathrm{mi}$. al E de San Miguel (Sonora) (Selander, 1960a); $20 \mathrm{mi}$. al NO de Nueva Casas Grandes (Selander, 1960a).

L. biguttata bivirgata: MÉxICo: Guanajuato: Guanajuato (Champion, 1892); Silao (Dugès, 1881 sub C. bivirgata; Dugès, 1889e sub C. alfredi; Champion, 1892; Vaurie, 1950; Selander, 1956, 1960a; Zaragoza-Caballero, 1999 sub C. alfredi); Tupátaro (Dugès, 1889e sub C. alfredi; Champion, 1892; Vaurie, 1950; Selander, 1956, 1960a; Zaragoza-Caballero, 1999 sub C. alfredi); 1 mi. al E de Villagrán (Selander, 1960a); México: 14 mi. al O de Toluca (Selander, 1960a).

Lytta (Paralytta) bipuncticollis Haag-Rutenberg, 1880

Lytta bipuncticollis Haag-Rutenberg, 1880: 36 (*)

TERRA TYPICA: No indicada en la descripción original; "Mexico" tras designación de lectotipo por Selander (1960a), que designa como lectotipo una macho con la etiqueta "Mexico, V. Heyd." de la colección Haag-Rutenberg del Zoologische Staatssammlung de Munich.

Cantharis bipuncticollis (Haag-Rutenberg, 1880): Beauregard, 1890: $492(*)$ 
DISTRIBUCIÓN GEOGRÁFICA: Endemismo mexicano conocido de los estados de Guerrero, Oaxaca y Veracruz.

MÉxICO: Guerrero: Amula (Champion, 1892; Selander, 1960a); Mochitlán (Champion, 1892; Selander, 1960a). Oaxaca: Oaxaca de Juárez (Champion, 1892; Selander, 1960a). Veracruz: Córdoba (Champion, 1892; Selander, 1960a); Xalapa (Champion, 1892; Selander, 1960a).

Lytta (Paralytta) canelas Selander, 1960

Lytta (Paralytta) canelas Selander, 1960a: 79 (*)

TERRA TYPICA: "Canelas, Durango". Holotipo en el British Museum of Natural History (Londres) (fide Selander, 1960a)

COMENTARIOS TAXONÓMICOS: Los ejemplares de la serie típica de $L$. canelas fueron identificados por Champion (1892) y por Vaurie (1950) como L. plumbea.

Distribución GeOGRÁFICA: Endemismo de México, sólo conocido de la localidad típica en Durango.

MÉXICo: Durango: Canelas (Champion, 1892 sub plumbea; Vaurie, 1950 sub plumbea; Selander, 1960a).

Lytta (Paralytta) corallifera Haag-Rutenberg, 1880

Lytta corallifera Haag-Rutenberg, 1880: 35 (*)

TERRA TYPICA: "Mexico" originalmente, "Mexico, Baden" tras designación de lectotipo. Selander (1960a) designa como lectotipo un ejemplar de la colección Haag-Rutenberg del Zoologische Staatsammlung de Munich.

Cantharis corallifera (Haag-Rutenberg, 1880): Beauregard, 1890: 492 (*)

DISTRIBUCIÓN GEOGRÁFICA: Endemismo mexicano.

MÉxICo: Hidalgo: Jacala (Selander, 1960a). San Luis Potosí: Sierra de San Miguelito (Champion, 1892; Selander, 1960a).

Lytta (Paralytta) cribrata LeConte, 1854

Lytta cribrata LeConte, 1854a: 447 (*)

TERRA TYPICA: No indicada en la descripción original; "vicinity of Eagle Pass, Texas" de acuerdo con el tipo (fide Selander, 1960a). Tipo en el Museum of Comparative Zoology (Harvard University, Cambridge, EEUU) (fide Selander, 1960a).

Cantharis intricata Champion, 1892: $441(*)$

TERRA TYPICA: "MeXICO, Villa Lerdo in Durango". El tipo está en el British Museum of Natural History (Londres) (fide Selander, 1960a) Cantharis pilsbryi Skinner, 1906: 217 (*)

TERRA TYPICA: "High Bridge, Pecos River, Texas" originalmente; "Guanajuato, Guanajuato" tras designación de neotipo (Selander, 1960a). Descripción original basada en una serie de 5 ejemplares. El neotipo está en la colección de la Philadelphia Academy of Sciences (fide Selander, 1960a)

Cantharis cribrata (LeConte, 1854): Horn, 1873: 111 (*)

Epicauta pilsbryi (Skinner, 1906): Borchmann, 1917: 80 (*)

Lytta intricata (Champion, 1892): Borchmann, 1917: 94 (*)

Lytta pilsbryi (Skinner, 1906): Denier, 1935b: 23 (*)

COMENTARIOS TAXONÓMicos: Dillon (1952) consideró

a C. pilsbryi como sinónimo de L. cribrata y Selander (1960a) añadió a $C$. intricata en su sinonimia. Denier (1935a: 162) publica el error tipográfico Lytta cribata.

DisTRIBUCIÓN GEOGRÁFICA: Especie extendida en Chihuahua y Durango y en una estrecha franja del sur de Texas (Selander, 1960a).

MÉxICo: Chihuahua: 12 mi. al NO de Gran Morelos (Selander, 1960a); Ojinaga (Selander, 1960a). Durango: Villa
Lerdo (Champion, 1892 sub C. intricata; Vaurie, 1950; Selander, 1960a).

\section{Lytta (Paralytta) deserticola Horn, 1870}

Lytta deserticola Horn, 1870: 90 (*)

TERRA TYPICA: “....south of Fort Whipple, Arizona”. El tipo estuvo inicialmente en la colección de la Academy of Natural Sciences of Philadelphia (fide Selander, 1960a), posteriormente transferido al Museum of Comparative Zoology de Harvard (EEUU).

Cantharis deserticola (Horn, 1870): Horn, 1873: 111 (*)

DISTRIBUCIÓN GEOGRÁFICA: Especie distribuida por el noroeste de México y sur de los EEUU.

MÉxICo: Sonora: Río Mayo (Selander, 1960a).

Lytta (Paralytta) ebenina (Dugès, 1877)

Cantharis ebenina Dugès, 1877: $66(*)$

TERRA TYPICA: No indicada en la descripción original; "San Andrés Tuxtla, Veracruz" tras designación como neotipo de un ejemplar hembra del British Museum of Natural History (Londres) (Selander, 1960a).

Lytta ebenina (Dugès, 1877): Borchmann, 1917: 93 (*)

COMENTARIOS TAXONÓMICOS: Dugès (1877) y Champion (1892) hacen referencia al nombre no disponible "Lytta ebenina" Chevrolat in Dejean, 1837: 246.

DisTRIBUCIÓN GEOGRÁFICA: Endemismo mexicano. Existe un ejemplar con etiqueta errónea de Panamá en la colección Haag-Rutenberg del Zoologische Staatssammlung de Munich (Selander, 1960a).

MÉxICo: Puebla: Amozoc (Selander, 1960a); Esperanza (Champion, 1892; Selander, 1960a); Izúcar de Matamoros (Champion, 1892; Selander, 1960a). Tabasco: La Venta, ruinas (Selander, 1960a). Tlaxcala: $4 \mathrm{mi}$. al E de Apizaco (Selander, 1960a); 5 mi. al E de Apizaco (Selander, 1960a); Carretera 18, en el límite entre Tlaxcala y Puebla (Selander, 1960a); $4 \mathrm{mi}$. al NO de Huamantla (Selander, 1960a); 2.5 mi. al O de El Carmen (Selander, 1960a). Veracruz: Boca del Monte (Dugès, 1889e); San Andrés Tuxtla (Champion, 1892; Selander, 1960a); Veracruz (Dugès, 1889e).

\section{Lytta (Paralytta) incompta Pinto, 1985}

Lytta (Paralytta) incompta Pinto, 1985: 335 (*)

TERRA TYPICA: "Mexico, Baja California Norte, 9 km NW Rancho Santa Inés: Lat. $29^{\circ} 46^{\prime} \mathrm{N}$, Long. $114^{\circ} 46^{\prime} \mathrm{W}, 550 \mathrm{~m}$ elev...”. El tipo está en la colección de la California Academy of Sciences (San Francisco, EEUU). De los 6 paratipos 2 se encuentran en la colección del Insituto de Biología de la Universidad Nacional Autónoma de México, 2 en la University of California at Riverside y otros 2 en la colección del College of Idaho (Pinto, 1985).

DISTRIBUCIÓN GEOGRÁFICA: Endemismo mexicano sólo conocido de la localidad típica en Baja California (Pinto, 1985)

MÉxICo: Baja California: 9 km NW Rancho Santa Inés: Lat. $2^{\circ} 46^{\prime} \mathrm{N}$, Long. $114^{\circ} 46^{\prime} \mathrm{W}$ (Pinto, 1985).

Lytta (Paralytta) koltzei Haag-Rutenberg, 1880

Lytta koltzei Haag-Rutenberg, 1880: 38 (*)

TERRA TYPICA: "Panama". De las tres hembras de la serie típica, Selander (1960a) designa como lectotipo una hembra con etiqueta "Panama, Koltze" de la colección del Zoologische Staatssammlung de Munich. La localidad típica es errónea (Selander, 1960a). 
Lytta koltzei var. minor Haag-Rutenberg, 1880: 38 (*)

TERRA TYPICA: "Mexico". Para resolver el problema taxonómico causado por los nombres $L$. $k$. var. minor y $L$. $k$. var. cyanescens designamos como lectotipo de $L$. $k$. var. minor al ejemplar de la serie típica de L. koltzei designado previamente por Selander (1960a) como lectotipo de $L$. $k$. var. cyanescens, una hembra con etiqueta "Mexico, V. Hyd." de la colección del Zoologische Staatssammlung de Munich.

Lytta humilis Haag-Rutenberg, 1880: 39 (*)

TERRA TYPICA: "Panama". La serie típica consta de dos machos y una hembra, Selander (1960a) designa como lectotipo un macho con etiqueta "Panama, Koltze" de la colección del Zoologische Staatssammlung de Munich. La localidad típica es errónea (Selander, 1960a).

Cantharis atrovirens Dugès, 1889e: 104 (*)

TERRA TYPICA: "México". El tipo está perdido (fide Selander, 1960a).

Cantharis humilis (Haag-Rutenberg, 1880): Beauregard, 1890: 494 (*)

Cantharis koltzei (Haag-Rutenberg, 1880): Beauregard, 1890: 494 (*)

Cantharis cyanescens Champion, 1892: 444 (*)

TERRA TYPICA: "Mexico". De las tres hembras de la serie típica de koltzei, Selander (1960a) designa como lectotipo de cyanescens una hembra con etiqueta "Mexico, V. Hyd." de la colección del Zoologische Staatssammlung de Munich.

Lytta atrovirens (Dugès, 1889e): Borchmann, 1917: 91 (*)

Lytta cyanescens (Champion, 1892): Borchmann, 1917: 93 (*)

COMENTARIOS TAXONÓMICOS: Varios autores consideran que Haag-Rutenberg (1880) describió dos variedades de L. koltzei (var. minor y var. cyanescens) (ver Champion, 1899 y Borchmann, 1917 por ejemplo), pero aunque Haag-Rutenberg utiliza dos nombres, minor y cyanescens, incluye ambos en la misma línea y con distinto tipo de letra. Por lo tanto Haag-Rutenberg tuvo la intención de usar uno sólo de los dos como nombre. El nombre que realmente usa Haag-Rutenberg es Lytta koltzei var. minor y menciona a continuación el nombre no disponible L. cyanescens Dejean, 1837: 246. Champion (1892) hace disponible el nombre de Cantharis cyanescens al proporcionar una descripción y Selander (1960) designa lectotipo para este taxón, aunque atribuye erróneamente el nombre de cyanescens a Haag-Rutenberg (1880) y el nombre de minor a Borchmann (1917). Para solventar el problema taxonómico expuesto y en cumplimiento del artículo 74.7 del Código Internacional de Nomenclatura Zoológica (CINZ, 2000), en este catálogo, designamos lectotipo de Lytta koltzei var. minor Haag-Rutenberg, 1880 al ejemplar designado previamente por Selander (1960a) como lectotipo de cyanescens, es decir una hembra con etiqueta "Mexico, V. Hyd." de la colección del Zoologische Staatssammlung de Munich. Tanto Beauregard (1890) como Champion (1892) mencionan también el nombre no disponible $L$. cyanescens Dejean, 1837: 246. Denier (1935a: 162) incluye el error tipográfico Lytta kolzei.

Selander (1960a) con la designación de lectotipos basada sobre el material disponible por Haag-Rutenberg, resuelve el problema koltzei-cyanescens-monilicornis y establece definitivamente la sinonimia de $C$. cyanescens con L. koltzei y la de C. monilicornis con L. plumbea. El problema era complicado ya que aunque Beauregard (1890) consideró con dudas a L cyanescens como sinónimo de L. koltzei, Champion (1892) incluyó en la sinonimia de L. koltzei a C. monilicornis, a la vez que trataba a $C$. cyanescens como especie independiente, pero incluyendo en su sinonimia a una de las variedades innominadas de C. monilicornis de Dugès. Borchmann (1917) también trató a $L$. cyanescens como especie diferente de L. koltzei, pero incluyendo en la sinonimia de L. koltzei a C. monilicornis

Selander (1960a) también considera con dudas a Cantharis atrovirens Dugès, 1889 en la sinonimia de $L$. koltzei, aunque el tipo está perdido y según Selander (1960a), la descripción, en particular la forma del espolón externo de la tibia posterior, no concuerda con la de L. koltzei. Mientras el tipo no aparezca, parece apropiado seguir el criterio de Selander (1960a) por lo que aquí consideramos a L. atrovirens como sinónimo de L. koltzei. El problema es complicado ya que Dugès (1886a: 582) utilizó por primera vez el nombre Cantharis atrovirens sin acompañarlo de una descripción y ZaragozaCaballero (1999) indica que en un manuscrito inédito, Dugès incluye este taxon como variedad de Cantharis monilicornis. Champion (1893), por otra parte, señala que la presencia de un espolón externo alargado en la tibia posterior, tal como indica Dugès en su descripción de Cantharis atrovirens, es característico de Linsleya suavissima.

DISTRIBUCIÓN GEOGRÁFICA: Endemismo mexicano, aunque parte de la serie típica está etiquetada erróneamente de Panamá (Selander, 1960a). Citado en el Distrito Federal, México, Michoacán, Morelos y Puebla. Selander (1960a) tras examinar los ejemplares, considera implícitamente que las citas de Champion (1892) de C. koltzei corresponden a L. plumbea.

MÉxICo: Distrito Federal: Ciudad de México (Dugès, 1889e sub C. atrovirens; Champion, 1892 sub C. cyanescens; Selander, 1960a); Tlalpan (Selander, 1960a). México: Real de Arriba, cerca de Temescaltepec (Selander, 1960a); Temescaltepec (Selander, 1960a). Michoacán: Pátzcuaro (Selander, 1960a); Quiroga (Zaragoza-Caballero, 1999 sub C. monilicornis var. atrovirens). Morelos: $6 \mathrm{mi}$. al $\mathrm{N}$ de Cuernavaca (Selander, 1960a); $9 \mathrm{~km}$ al S de Tres Cumbres (Selander, 1960a); Tres Marías, Tres Cumbres (Selander, 1960a). Puebla: Esperanza (Champion, 1892 sub C. cyanescens; Selander, 1960a).

Lytta (Paralytta) lecontei Heyden, 1890

Lytta dichroa LeConte, 1853: 332 (non Lytta dichroa Fischer de Waldheim, 1824: 230) $\left(^{*}\right)$

TERRA TYPICA: "Mexican Boundary". Holotipo en el Museum of Comparative Zoology (fide Selander, 1960a).

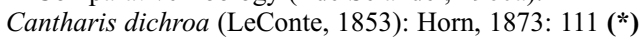

Lytta lecontei Heyden, 1890: 99 (nombre de substitución para Lytta dichroa LeConte, 1853) (*)

Cantharis lecontei (Heyden, 1890): Champion, 1899: 181 (*)

Epicauta lecontei (Heyden, 1890): Borchmann, 1917: 77 (*) 
COMENTARIOS TAXONÓMICOS: Borchmann (1917) incluye simultáneamente a esta especie en los géneros Lytta y Epicauta.

DISTRIBUCIÓN GEOGRÁFICA: Especie extendida en el oriente de Texas, citada sin precisión en México (Horn, 1873), con un único registro confirmado en Tamaulipas (Selander, 1960a).

MÉXICO: Tamaulipas: Río Rico (Selander, 1960a).

Lytta (Paralytta) magister Horn, 1870

Lytta magister Horn, 1870: $90(*)$

TERRA TYPICA: "Owen's Valley, California". Holotipo inicialmente en la colección de la Academy of Natural Sciences of Philadelphia (fide Selander, 1960a), posteriormente transferido al Museum of Comparative Zoology de la University of Harvard (EEUU). Cantharis magister (Horn, 1870): Horn, 1873: $106(*)$

COMENTARIOS TAXONÓMICOS: Incluida en el subgénero Paralytta por Selander (1960a).

DisTRIBUCIÓN GEOGRÁFICA: Distribuida en zonas áridas del suroeste de Estados Unidos con un único registro en Sonora (Selander, 1958).

MÉXICo: Sonora: Volcán Cerro Colorado, unas $35 \mathrm{mi}$. al O-NO de Sonoyta (Selander, 1958; 1960a; Werner et al., 1966).

Lytta (Paralytta) margarita (Fall, 1901)

Cantharis margarita Fall, 1901b: 300 (*)

TERRA TYPICA: "Santa Margarita Island, Lower California". La descripción original se basó en un único ejemplar macho de la colección C. Fuchs (fide Selander, 1960a), que estaría en la colección de la California Academy of Sciences. Sin embargo H.B. Leech (en Selander, 1960a) señala que el ejemplar no está en esa colección, por lo que posiblemente se perdería en el incendio de San Francisco junto con la mayor parte de la colección Fuchs (Selander, 1960a).

Lytta margarita (Fall, 1901): Borchmann, 1917: 96 (*)

COMENTARIOS TAXONÓMICOS: Incluida en el subgénero Paralytta por Selander (1960a). Es posible que esta especie entre dentro de la variabilidad de L. vulnerata.

Distribución GEOGRÁfICA: Sólo conocida de Baja California Sur.

MÉxICo: Baja California Sur: Isla Santa Margarita (Fall, 1901b; Selander, 1960a); Guerrero Negro (Pinto, 1985).

Lytta (Paralytta) melaena LeConte, 1858

Lytta melaena LeConte, 1858b: 76 (*)

TERRA TYPICA: "Sonora". Tipo en el Museum of Comparative Zoology (Harvard University, Cambridge, EEUU) (fide Selander, 1960a).

Cantharis melaena (LeConte, 1858): Horn, 1873: 105 (*)

COMENTARIOS TAXONÓMICOS: Incluida en el subgénero Paralytta por Selander (1960a).

DisTRIBUCIÓN GEOGRÁFICA: Exclusiva de la península de Baja California, Sonora y el suroeste de EEUU (Selander, 1960a).

MÉXICo: Baja California Sur: San José de Gracia (Horn, 1896; Selander, 1960a); San José del Cabo (Fall, 1901b; Selander, 1960a); Isla Santa Margarita (Horn, 1896; Selander, 1960a). Sonora: Sin precisar (LeConte, 1858b; Selander, 1960a); frontera entre Arizona y Sonora (Horn, 1873, 1896).

Lytta (Paralytta) michoacanae (Champion, 1892)

Cantharis michoacanae Champion, 1892: 447 (*)

TERRA TYPICA: "MeXICO, Huetamo and Tacambaro in Michoacan...". El tipo, de Huétamo, está en la colección del British Museum of Natural History (Londres) (fide Selander, 1960a).

Lytta michoacanae (Champion, 1892): Borchmann, 1917: 96 (*)

COMENTARIOS TAXONÓMICOS: Incluida en el subgénero Paralytta por Selander (1960a).

DisTRIBUCIÓN GEOGRÁFICA: Endemismo mexicano localizado en la cuenca del Río Balsas. Citado en Guerrero y Michoacán.

MÉXICo: Guerrero: Iguala (Selander, 1960a); $30 \mathrm{~km}$ al S de Iguala (Selander, 1960a); Mezcala (Selander, 1954b; Selander, 1960a); 5 mi. al NNE de Mezcala (Selander, 1954b; Selander, 1960a); $9 \mathrm{~km}$ al N de Zumpango del Río (Selander, 1960a). Michoacán: Huetamo (Champion, 1892; Selander, 1960a); Tacámbaro de Codallos (Champion, 1892; Selander, 1960a).

Lytta (Paralytta) nitidicollis (LeConte, 1851)

Cantharis nitidicollis LeConte, 1851: 160 (*)

TERra tYPICA: "San Diego". Holotipo en el Museum of Comparative Zoology (fide Selander, 1960a).

Lytta nitidicollis (LeConte, 1851): LeConte, 1853: 332 (*)

COMENTARIOS TAXONÓMICOS: Incluida en el subgénero Paralytta por Selander (1960a).

DisTRIBUCIÓN GEOGRÁFICA: Especie casi exclusiva de California, posiblemente amenazada (Papp, 1960), que cuenta con un único registro sin precisar en Baja California (Fall, 1901b; Selander, 1960a).

MÉXICo: Baja California: Sin precisar (Fall, 1901b).

Lytta (Paralytta) plumbea Haag-Rutenberg, 1880

Lytta plumbea Haag-Rutenberg, 1880: 38 (*)

TERRA TYPICA: "Mexico". Selander (1960a) designa como lectotipo una hembra de la colección Haag-Rutenberg del Zoologische Staatssammlung de Munich.

Cantharis monilicornis Dugès, 1881: 142 (*)

TERRA TYPICA: "Uruápan", originalmente; "Guanajuato, [Guanajuato]" tras designación como neotipo de una hembra de la colección del British Museum of Natural History (Londres) (Selander, 1960a).

Cantharis plumbea (Haag-Rutenberg, 1880): Beauregard, 1890: 496 (*)

COMENTARIOS TAXONÓMICOS: Beauregard (1890) consideró a C. monilicornis como una especie válida, pero Champion $(1892,1899)$ consideró a C. monilicornis como sinónimo de L. cyanescens. Selander (1960a) tras designar neotipo transfiere $C$. monilicornis a la sinonimia de L. plumbea.

DISTRIBUCIÓN GEOGRÁFICA: Endemismo mexicano, citado en el Distrito Federal, Guanajuato, México y Michoacán. Algunas de las citas de L. cyanescens de Champion (1892), corresponden a L. koltzei, mientras que otras, junto con la mayoría de las de L. koltzei, correspon- 
den a L. plumbea tal como indica implícitamente Selander (1960a) tras examinar los ejemplares. Las citas de Champion (1892) de Canelas (Durango) corresponden a $L$. canelas (Selander, 1960a).

MÉxICo: Distrito Federal: Ciudad de México (Champion, 1892 sub C. koltzei; Selander, 1960a); Valle de México (Dugès, 1889 e sub C. monilicornis; Champion, 1892 sub C. koltzei; Zaragoza-Caballero, 1999 sub C. monilicornis). Guanajuato: Guanajuato (Dugès, 1889e sub C. monilicornis; Champion, 1892 sub C. koltzei; Selander, 1960a); Moroleón (Dugès, 1889 sub C. monilicornis; Champion, 1892 sub C. koltzei; Selander, 1960a). México: Toluca (Champion, 1892 sub C. koltzei; Selander, 1960a); 1.8 mi. al SE de Toluca (Selander, 1960a). Michoacán: Carapan (Dugès, 1889; Selander, 1960a); Morelia (Zaragoza-Caballero, 1999 sub C. monilicornis); Pátzcuaro (Dugès, 1889e; Selander, 1960a); Quiroga (Dugès, 1889e sub C. monilicornis; Champion, 1892 sub C. cyanescens; Selander, 1960a); Tancítaro (Selander, 1960a); Uruápan del Progreso (Dugès, 1881, 1889e sub C. monilicornis; Champion, $1892 \mathrm{sub}$ C. koltzei; Selander, 1960a; Zaragoza-Caballero, 1999 sub C. monilicornis).

\section{Lytta (Paralytta) proteus Haag-Rutenberg, 1880}

Lytta proteus Haag-Rutenberg, 1880: 37 (*)

TERRA TYPICA: "Mexico". Selander (1960a) designa como lectotipo un ejemplar de la colección Haag-Rutenberg del Zoologische Staatsammlung de Munich.

Cantharis sobrina Dugès, 1881: 143 (*)

TERRA TYPICA: "Pénjamo". Selander (1960a), tras indicar que el tipo está perdido, designa como neotipo un macho de de Pénjamo, Guanajuato, en el British Museum of Natural History (Londres). Sin embargo, Vázquez \& Zaragoza (1979) indican como tipo el ejemplar $\mathrm{n}^{\circ} 1373$ (IBUNAM 020) de la colección del Instituto de Biología de la Universidad Nacional Autónoma de México.

Cantharis bipartita Dugès, $1889 \mathrm{e}: 106$ (*)

TERRA TYPICA: "Chilpancingo"; "Rincón, Guerrero" tras designación de un macho como neotipo, en el British Museum of Natural History (Londres) (Selander, 1960a).

Cantharis proteus (Haag-Rutenberg, 1880): Beauregard, 1890: 496 (*) Lytta proteus ab. haagrutenbergi Kaszab, 1958: 189 (nombre no disponible) (*)

Lytta proteus ab. rutenbergi Kaszab, 1958: 189 (nombre no disponible) (*) Lytta proteus ab. haagi Kaszab, 1958: 189 (nombre no disponible) (*)

COMENTARIOS TAXONÓMICOS: Beauregard (1890) trata a C. sobrina como especie válida. Incluida en el subgénero Paralytta por Selander (1960a). Los nombres propuestos por Kaszab (1958) como sustitutos de nombres inexistentes de Haag-Rutenberg (1880) no están disponibles.

DisTRIBUCIÓN GEOGRÁfICA: Endemismo mexicano citado en Colima, Distrito Federal, Guanajuato, Guerrero, Jalisco, México, Michoacán, Morelos, Nayarit, Oaxaca y Puebla.

MÉxICo: Colima: Colima (Selander, 1960a); Tonila (Champion, 1892; Selander, 1960a). Distrito Federal: Ciudad de México (Champion, 1892; Selander, 1960a); Tlalpan (Selander, 1960a). Guanajuato: Pénjamo (Dugès, 1881, 1889e sub C. sobrina; Champion, 1892; Selander, 1960a; ZaragozaCaballero, 1999 sub C. sobrina). Guerrero: Acahuizotla (Champion, 1892; Selander, 1960a); Chilpancingo (Dugès, 1889e sub C. bipartita; Champion, 1892; Selander, 1960a); Garcidueña (Dugès, 1889e sub C. sobrina; Champion, 1892;
Selander, 1960a; Vázquez \& Zaragoza, 1979); Hacienda de la Imagen (Champion, 1892; Selander, 1960a); Rincón (Selander, 1960a); Taxco (Selander, 1960a); Tierra Colorada (Champion, 1892; Selander, 1960a); Venta del Peregrino (Champion, 1892; Selander, 1960a). Jalisco: Guadalajara (Selander, 1960a); Zapopan (Selander, 1960a). México: (San Juan) Teotihuacán (Selander, 1960a); Temescaltepec (Selander, 1960a). Michoacán: $7 \mathrm{mi}$. al S de Arteaga (Selander, 1960a); Jungapeo (Selander, 1960a); Tacámbaro (Champion, 1892; Selander, 1960a). Morelos: Alpuyeca (Selander, 1960a); Tepoztlán (Selander, 1960a). Nayarit: 4 mi. al S de Santa Isabel (Selander, 1960a); 4 mi. al NO de Tepic (Selander, 1960a). Oaxaca: Huajuapan de León (Selander, 1960a). Puebla: Izúcar de Matamoros (Champion, 1892; Selander, 1960a); Puebla (Selander, 1960a).

Lytta (Paralytta) quadrimaculata (Chevrolat, 1834)

Cantharis quadrimaculata Chevrolat, 1834b: fasc. 4, $\mathrm{n}^{\circ} 79$ (sin paginar) $(*)$

TERRA TYPICA: "Orixaba". Localización del tipo desconocida (fide Selander, 1960a).

Cantharis octomaculata? Peñafiel Barranco, 1866: $225(*)$

TERRA TYPICA: “...cerca del mineral del Chico y Atotonilco el Grande...", originalmente; "Pachuca, 8000 ft., Hidalgo" al designar neotipo (Selander, 1960a). Serie típica original destruida (fide Selander, 1960a), neotipo macho con etiqueta "Pachuca, $8000 \mathrm{ft}$., Hidalgo, July 1937, K. Maehler collection" de la colección de la California Academy of Sciences (San Francisco, EEUU) (Selander, 1960a).

Cantharis fasciolata Jiménez, 1866b: 253 (*)

TERRA TYPICA: No señalada originalmente; "Mexico" por designación como neotipo de un ejemplar macho con etiqueta "Mexico, Sallé collection" de la colección del British Museum of Natural History (Londres) (Selander, 1960a).

Cantharis bifasciatus Dugès, 1869a: 106 (*)

Terra typica: “... pueblo del Puente perteneciente al Mineral del Chico, en Pachuca, y cerca de la capital de México"; "Pachuca, 8000 ft., Hidalgo" al designar neotipo (Selander, 1960a). Aunque en la colección del Instituto de Biología de la Universidad Nacional Autónoma de México existen ejemplares que podrían corresponder a la serie típica (fide Selander, 1960a), Selander (1960a) designa como neotipo de C. bifasciata el mismo ejemplar que designó como neotipo de C. octomaculata de la colección de la California Academy of Sciences (San Francisco, EEUU).

Lytta quadrimaculata (Chevrolat, 1834): Borchmann, 1917: 98 (*)

Lytta octomaculata (Peñafiel Barranco, 1866): Denier, 1940: 423 (*)

COMENTARIOS TAXONÓMICOS: Incluida en el subgénero Paralytta por Selander (1960a). Dugès (1870) establece la sinonimia de $C$. bifasciata y $C$. fasciolata con $L$. quadrimaculata (ver también Zaragoza-Caballero, 1999: Cantharis quadrimaculata var. bifasciata), mientras que Champion (1892) consideró a C. bifasciata como una variedad de L. quadrimaculata. Denier (1935a) considera a $C$. fasciolata como variedad de L. bifasciata. Además Chevrolat (1834b), Dugès (1869a) y Beauregard (1890) incluyen en la sinonimia los nombres no disponibles Cantharis disparicornis Chevrolat y Lytta mexicana Klug in Dejean, 1837: 246, mientras que Dugès (1889) utiliza el nombre no disponible Lydus bifasciatus Sturm, 1843: 173. Champion (1892) recoge los nombres no disponibles anteriores e incluye con dudas en la sinonimia a Cantharis alemani Dugès, nom- 
bre que Selander (1960a) incluye en la sinonimia de $L$. biguttata.

DisTRIBUCIÓN GEOGRÁFICA: Especie casi exclusiva de México con dos registros en Texas (EEUU), muy cerca de la frontera (Horn, 1873; Fall, 1901b; Dillon, 1952; Selander, 1960a). Citada en Aguascalientes, Chihuahua, Coahuila, Colima, Distrito Federal, Durango, Guanajuato, Hidalgo, Jalisco, México, Michoacán, Nuevo León, Puebla, Querétaro, San Luis Potosí, Tlaxcala, Veracruz y Zacatecas. Selander (1960a) indica que los Estados asignados por Vaurie (1950) a las localidades Pueblo del Puente y Almolonga (Nayarit y Oaxaca, respectivamente) son erróneas y corresponden a Hidalgo y Veracruz respectivamente.

MÉxICo: Aguascalientes: El Retorno, $10 \mathrm{mi}$. al E de Aguascalientes (Selander, 1960a); $4.1 \mathrm{mi}$. al SO de Aguascalientes (Selander, 1960a); Aguascalientes (Champion, 1892; Vaurie, 1950). Chihuahua: Salaices (Selander, 1960a). Coahuila: 15 mi. al S de Arteaga (Selander, 1960a). Colima: Zapotlán (Champion, 1892; Vaurie, 1950). Distrito Federal: Chapultepec (Selander, 1960a); Ciudad de México (Dugès, 1869a sub C. bifasciatus; Dugès, 1889e; Champion, 1892; Vaurie, 1950); Coapa (Selander, 1960a); Guadalupe (Vaurie, 1950; Selander, 1960a); San Ángel (Vaurie, 1950; Selander, 1960a); Santa Fé (Vaurie, 1950; Selander, 1960a); Tlalpan (Vaurie, 1950; Selander, 1960a). Durango: Durango (Selander, 1960a); 6 mi. al S de Durango (Selander, 1960a); San Lucas, Ocampo (Vaurie, 1950; Selander, 1960a); Sierra de Durango (Selander, 1960a); Ciudad Lerdo (Champion, 1892; Vaurie, 1950; Selander, 1960a). Guanajuato: Guanajuato (Dugès, 1889e; Champion, 1892; Selander, 1960a). Hidalgo: Atotonilco El Grande (Peñafiel Barranco, 1866 sub Cantharis octomaculata?; Selander, 1960a); Mineral del Chico (Peñafiel Barranco, 1866 sub Cantharis octomaculata?; Selander, 1960a); Pachuca (Vaurie, 1950; Selander, 1960a); 16 mi. al E de Pachuca (Selander, 1960a); Pueblo del Puente, Mineral de Chico (Dugès, 1869a sub C. bifasciatus; Dugès, 1889e; Champion, 1892; Vaurie, 1950; Selander, 1960a). Jalisco: Guadalajara (Champion, 1892; Vaurie, 1950; Selander, 1960a); La Punta (Selander, 1960a); 4 mi. al O de Mazamitla (Selander, 1960a); Ojuelos de Jalisco (Selander, 1960a); Sayula (Champion, 1892; Vaurie, 1950; Selander, 1960a); Zapopan (Vaurie, 1950; Selander, 1960a); Zapotlán, Ciudad Guzmán (Selander, 1960a). México: Amecameca (Selander, 1960a); Atlacomulco (Selander, 1960a); Chapingo (Selander, 1960a); Paso de Cortés, ladera occidental (Selander, 1960a); 4 mi. al N de Ixtlahuaca (Selander, 1960a); $10 \mathrm{mi}$. al NO de Ixtlahuaca (Selander, 1960a); San Rafael (Selander, 1960a); Temescaltepec (Selander, 1960a); Tlanepantla (Vaurie, 1950; Selander, 1960a); Toluca (Champion, 1892; Vaurie, 1950); 4 mi. al E de Toluca (Selander, 1960a); $1.8 \mathrm{mi}$. al SE de Toluca (Selander, 1960a); 26 mi. al S de Toluca (Selander, 1960a); 14 mi. O Toluca (Selander, 1960a); $14 \mathrm{mi}$. al NO de Toluca (Selander, 1960a); 15 mi. al NO de Toluca (Selander, 1960a); Zinacantepec (Selander, 1960a). Michoacán: Morelia (Dugès, 1889e; Champion, 1892; Vaurie, 1950; Selander, 1960a); Tacámbaro (Champion, 1892; Vaurie, 1950; Selander, 1960a); Tancítaro (Selander, 1960a). Nuevo León: $15 \mathrm{mi}$ al SO de China (Selander, 1960a). Puebla: $5 \mathrm{mi}$. al S de Acatzingo
(Selander, 1960a); Amozoc (Selander, 1960a); Atlixco (Selander, 1960a); Esperanza (Champion, 1892; Vaurie, 1950; Selander, 1960a); Izúcar de Matamoros (Champion, 1892; Vaurie, 1950; Selander, 1960a); Puebla (Vaurie, 1950); Techamachalco (Selander, 1960a); Tehuacán (Selander, 1960a); 5 mi. al NE de Teziutlán (Selander, 1960a). Querétaro: 10 mi. al E de San Juan del Río (Selander, 1960a). San Luis Potosí: Hacienda de Bleados [Bledos según Selander \& Vaurie, 1962] (Selander, 1960a); San Luis Potosí (Champion, 1892; Selander, 1960a). Tlaxcala: $4 \mathrm{mi}$. al E de Apizaco (Selander, 1960a); 10 mi. al E de Apizaco (Selander, 1960a); 2.5 mi. O El Carmen (Selander, 1960a); 4 mi. al NO de Huamantla (Selander, 1960a); 9 mi. al E de Huamantla (Selander, 1960a). Veracruz: Almolonga (Champion, 1892; Vaurie, 1950; Selander, 1960a); Xalapa (Vaurie, 1950; Selander, 1960a); Limón (Selander, 1960a); Orizaba (Chevrolat, 1834b; Dugès, 1869a; Champion, 1892; Vaurie, 1950; Selander, 1960a); Perote (Champion, 1892; Vaurie, 1950; Selander, 1960a); Veracruz (Dugès, 1889e; Champion, 1892; Zaragoza-Caballero, 1999 sub C. q. var. bifasciata). Zacatecas: Ojocaliente (Selander, 1960a).

\section{Lytta (Paralytta) scitula (Champion, 1892)}

Cantharis scitula Champion, 1892: 446 (*)

Terra tyPica: "MeXICo, Chilpancingo in Guerrero 4600 feet...". El tipo, único ejemplar de la serie típica, está en el British Museum of Natural History (Londres) (fide Selander, 1960a).

Cantharis signaticollis Champion, 1892: 446 (*)

Terra tyPiCA: "MeXico, Matamoros Izúcar...". El tipo, un macho, junto con los otros 3 ejemplares de la serie típica, está en el British Museum of Natural History (Londres) (fide Selander, 1960a).

Lytta scitula (Champion, 1892): Borchmann, 1917: 98 (*)

Lytta signaticollis (Champion, 1892): Borchmann, 1917: 98 (*)

COMENTARIOS TAXONÓMICOS: Selander (1960a) establece la sinonimia de $L$. signaticollis con L. scitula. Incluida en el subgénero Paralytta por Selander (1960a).

DisTRIBUCIÓN GEOGRÁfICA: Endemismo mexicano.

MÉxICo: Guerrero: Chilpancingo de los Bravos (Champion, 1892; Selander, 1960a). Oaxaca: Nochixtlán (Selander, 1960a). Puebla: Amozoc (Selander, 1960a); Atlixco (Selander, 1960a); Izúcar de Matamoros (Champion, $1892 \mathrm{sub}$ C. signaticollis; Selander, 1960a).

\section{Lytta (Paralytta) scituloides Selander, 1960}

Lytta (Paralytta) scituloides Selander, 1960a: 67 (*)

TERRA TYPICA: "[Villa] Allende, [Nuevo León]". Holotipo en la colección del United States National Museum of Natural History (Washington D.C.) (fide Selander, 1960a).

COMENTARIOS TAXONÓMICOS: Selander (1960a) señala que $L$. scituloides difiere relativamente poco de $L$. variabilis, y que tal vez podría constituir una raza geográfica de L. variabilis en lugar de una especie diferenciada.

DistribuCión GEOGRÁFICA: Endemismo mexicano. Citado en Coahuila y Nuevo León.

MÉxICo: Coahuila: Saltillo (Selander, 1960a). Nuevo León: Apodaca (Selander, 1960a); Cola de Caballo cerca de Monterrey (Selander, 1960a); Monterrey (Selander, 1960a); Villa Allende (Selander, 1960a); Villa de Santiago (Selander, 1960a). 
Lytta (Paralytta) sonorae Van Dyke, 1947

Lytta sonorae Van Dyke, 1947: $156(*)$

Terra TYPICA: "Carbo, Sonora, Mexico...". Holotipo, n5868, y paratipos en la colección California Academy of Sciences (San Francisco) (fide Van Dyke, 1947; Selander, 1960a).

COMENTARIOS TAXONÓMICOS: Incluida en el subgénero Paralytta por Selander (1960a).

DisTribuCión GEOGRÁfICA: Endemismo mexicano sólo conocido de la localidad típica en Sonora.

MÉxICo: Sonora: Carbó (Van Dyke, 1947; Selander, 1960a).

\section{Lytta (Paralytta) variabilis (Dugès, 1869) \\ Cantharis variabilis Dugès, 1869a: $111\left({ }^{*}\right)$ \\ TERRA TYPICA: “...cerros de Guanajuato" originalmente; "Guanajuato" tras designación de neotipo. Selander (1960a) indica que el tipo se perdió y designa como neotipo una hembra de prono- to negro de "Guanajuato" en el British Museum of Natural History. Vázquez \& Zaragoza (1979), sin embargo, indican como tipo el ejemplar $n^{\circ} 798$ (IBUNAM 004) de la colección del Instituto de Biología de la Universidad Nacional Autónoma de México. \\ Lytta variabilis (Dugès, 1869): Borchmann, 1917: $100(*)$}

COMENTARIOS TAXONÓMICOS: Selander (1960a) indica que los ejemplares del oeste de Jalisco y sobre todo del oeste del Estado de México (Temascaltepec) presentan un grado de diferenciación notable con respecto a las demás poblaciones, incluidas las de Toluca (México). Horn (1885) y Beauregard (1890) consideraron a $C$. variabilis como sinónimo de $C$. puberula, un criterio que no ha sido seguido por otros autores. Champion (1892) menciona en la sinonimia de esta especie el nombre no disponible Lydus coracinus Sturm, 1843: 173.

DISTRIBUCIÓN GEOGRÁFICA: Endemismo mexicano, con una distribución que incluye desde Chihuahua hasta México y Puebla (Selander, 1960a). Citado en los estados de Chihuahua, Durango, Guanajuato, Hidalgo, Jalisco, México, Puebla, Querétaro, San Luis Potosí y Zacatecas.

MÉXICo: Chihuahua: Santa Bárbara (Selander, 1960a). Durango: Durango (Selander, 1960a); Francisco Madero (Selander, 1960a); Guadalupe Victoria (Selander, 1960a); Nombre de Dios (Selander, 1960a). Guanajuato: Guanajuato (Dugès, 1869a; Dugès, 1889e; Champion, 1892; Selander, 1960a; Zaragoza-Caballero, 1999); 14 mi. al S de San Luis de la Paz (Selander, 1960a); San Miguel Allende (Selander, 1960a); Tupátaro (Dugès, 1889e; Champion, 1892; Selander, 1960a; Vázquez \& Zaragoza, 1979). Hidalgo: Jacala (Selander, 1960a); Pachuca (Selander, 1960a). Jalisco: Encarnación de Díaz (Selander, 1960a); 12 mi. al S de Encarnación de Díaz (Selander, 1960a); $96 \mathrm{~km}$ al SO de Guadalajara (Selander, 1960a); La Punta (Selander, 1960a). México: $20 \mathrm{mi}$. al SO de Acambay (por carretera) (Selander, 1960a); 6 mi. al N de Aculco (Selander, 1960a); Atlacomulco (Selander, 1960a); Temascaltepec (Selander, 1960a). Puebla: Puebla (Selander, 1960a). Querétaro: Km 320 near Hacienda Balvanera (Selander, 1960a). San Luis Potosí: San Luis Potosí (Champion, 1892; Selander, 1960a). Zacatecas: $17 \mathrm{mi}$. al N de Fresnillo (Linsley et al., 1956; Selander, 1960a); 1.5 mi. al S de
Fresnillo (Linsley et al., 1956; Selander, 1960a); 9 mi. al S de Fresnillo (Linsley et al., 1956; Selander, 1960a); Guadalupe (Selander, 1960a); 5 mi. al S de Sombrerete (Selander, 1954b; Selander, 1960a); 2 mi. al S de Trancosa (Selander, 1960a).

Lytta (Paralytta) vulnerata (LeConte, 1851)

Lytta (Paralytta) vulnerata vulnerata (LeConte, 1851)

Cantharis vulnerata LeConte, 1851: 159 (*)

TERRa tYPICA: "San Diego". Tipo en el Museum of Comparative Zoology (Harvard University, Cambridge, EEUU) (fide Selander, 1960a)

Lytta vulnerata (LeConte, 1851): LeConte, 1853: 331 (*)

Cantharis angulicollis Dugès, 1889e: $105(*)$

TERRA TYPICA: "Baja California"; neotipo designado por Selander (1960a) en la colección Dugès del Instituto de Biología de la Universidad Nacional Autónoma de México. Vázquez \& Zaragoza (1979) indican que el tipo es el ejemplar $n^{\circ} 2796$ (IBUNAM 030).

COMENTARIOS TAXONÓMICOS: Especie con dos subespecies, L. v. vulnerata y L. v. cooperi LeConte, 1854 (Lytta cooperi LeConte, 1854b: 18), con una complicada historia taxonómica. Horn (1873: 106) considera a $L$. cooperi como una variedad de L. vulnerata (como Cantharis vulnerata var. cooperi); posteriormente, Beauregard (1890), la incluye en la sinonimia de $L$. vulnerata; Fall (1901b) la vuelve a considerar a nivel específico, mientras que Knowlton \& Taylor (1952), seguidos por Selander (1960a), aunque este último no con carácter formal, la tratan como subespecie de L. vulnerata. Incluida en el subgénero Paralytta por Selander (1960a). Selander (1960a: 131) desliza el error tipográfico Cantharis anguicollis.

DistribUCión GEOGRÁFICA: Ampliamente distribuida en la región occidental de EEUU, con un único registro en Baja California (Selander, 1960a). En México únicamente se encuentra la subespecie típica.

MÉxICo: Baja California: Sin precisar (Dugès, 1889e; Selander, 1960a).

Subgénero Poreospasta Horn, 1868

Poreospasta Horn, 1868: 139 (*)

ESPECIE TIPO: Poreospasta polita Horn, 1868 (= Nomaspis sublaevis Horn, 1868), por monotipia.

CONTENIDO Y DISTRIBUCIÓN: El subgénero incluye unas 25 especies, al menos 4 de ellas en Asia y el resto en América del Norte, desde el noroeste de México hasta el suroeste del Canadá (Selander, 1960a). Poreospasta ha sido tratado por diversos autores (Borchmann, 1917) como un género independiente.

Selander (1960a) divide el subgénero en cinco grupos de especies, de los cuales únicamente dos se encuentran en América: los grupos de L. moerens (LeConte, 1851) y de L. stygica (LeConte, 1851), cada uno de ellos con una especie presente en México. Las dos especies presentes en México son: Lytta (Poreospasta) auriculata Horn, 1870 y Lytta (Poreospasta) childii LeConte, 1857. Además de estas especies, podrían encontrarse también, ya que se se 
han citado en California (EEUU) muy cerca de la frontera (Selander, 1960a), las siguientes: Lytta (Poreospasta) aeneipennis (LeConte, 1851); Lytta (Poreospasta) crotchi (Horn, 1874); Lytta (Poreospasta) insperata (Horn, 1874); Lytta (Poreospasta) lugens (LeConte, 1851); Lytta (Poreospasta) moerens y Lytta (Poreospasta) stygica. La presencia posible de L. stygica en México ha sido también señalada en el listado de Blackwelder (1945).

\section{Lytta (Poreospasta) auriculata Horn, 1870}

Lytta auriculata Horn, 1870: 91 (*)

TERRA TYPICA: "Kern River region of California...". Holotipo inicialmente en la colección de la Academy of Natural Sciences of Philadelphia (fide Selander, 1960a), posteriormente transferido al Museum of Comparative Zoology de Harvard (EEUU).

Cantharis auriculata (Horn, 1870): Horn, 1873: 113 (*)

COMENTARIOS TAXONÓMICOS: Especie con varabilidad geográfica marcada en la coloración (Van Dyke, 1929).

DiSTRIBUCIÓN GEOGRÁFICA: Su distribución incluye una amplia región del suroeste de los EEUU con registros en Baja California (Fall, 1901b; Selander, 1960a).

MÉxICo: Baja California: Playa Metero (Selander, 1960a).

\section{Lytta (Poreospasta) childii LeConte, 1857 \\ Lytta childii LeConte, 1857: 52 \\ TERRA TYPICA: San Francisco, California (fide Selander, 1960a) \\ Holotipo en el Museum of Comparative Zoology (Harvard \\ University, Cambridge, EEUU) (fide Selander, 1960a) \\ Cantharis childii (LeConte, 1857): Horn, 1873: 108 (*) \\ Lytta maculicollis Van Dyke, 1929: 130 (non Klug, 1825) (*) \\ TERRA TYPICA: "near Panoche Creek in western Fresno Co., Calif." \\ Holotipo, no. 2602, en la California Academy of Sciences (fide Van Dyke, 1929; Selander, 1960a)}

COMENTARIOS TAXONÓMICOS: Selander (1960a) establece la sinonimia de L. maculicollis con L. childii, de no ser así las poblaciones de California meridional y Baja California, requerirían un nombre nuevo.

DisTRIBUCIÓN GEOGRÁFICA: Ampliamente distribuida en California, con algunos registros en Baja California (Fall, 1901b; Selander, 1960a).

MÉxICo: Baja California: San Julio (Horn, 1894; Selander, 1960a). Baja California Sur: Isla Santa Margarita (Horn, 1894; Selander, 1960a).

Tribu Meloini Blanchard, 1845

Méloeites Blanchard, 1845: 43 (*)

Género tipo: Meloe Linnaeus, 1758.

CONTENIDO Y DISTRIBUCIÓN: La tribu tiene una distribución muy amplia en el Planeta. Hasta la década de 1960 únicamente incluía especies con élitros reducidos, aunque en la actualidad se incluyen géneros muy diversos, con élitros reducidos o bien desarrollados (Bologna \& Pinto, 1998). En América estarían presentes 5 géneros: Epispasta Selander, 1960, Lyttomeloe Denier, 1920 ,
Meloe, Spastomeloe Selander, 1985b y Spastonyx, de los cuales Meloe y Spastonyx se encuentran en México (Pinto \& Bologna, 1999). Sin embargo el estudio filogenético de Bologna \& Pinto (2001) no apoya la inclusión de muchos de esos géneros dentro de la tribu Meloini. Selander (1985b) elevó Eurymeloe al estatus genérico, pero en obras posteriores sigue tratándose como un subgénero de Meloe (Pinto \& Bologna, 1999; Bologna \& Pinto, 2001)

Género Meloe Linnaeus, 1758

Meloe Linnaeus, 1758: 419 (*)

ESPECIE TIPO: Meloe proscarabaeus Linnaeus, 1758, por designación subsecuente de Latreille (1810: 430).

CONTENIDO Y DISTRIBUCIÓN: El género está representado por 153 especies repartidas en 16 subgéneros, que se distribuyen por la región holártica, con extensiones en África subsahariana y en el sureste de Asia (Bologna \& Pinto, 1998, 2002). En América del Norte se conocen 24 especies pertenecientes a 3 subgéneros, 18 de ellas al subgénero Meloe, 5 a Treiodous y 1 a Eurymeloe Reitter, 1911 (Pinto \& Bologna, 1999). Los 5 representantes mexicanos del género se agrupan en los subgéneros Meloe y Treiodous (Pinto \& Selander, 1970).

Subgénero Meloe Linnaeus, 1758

Meloe Linnaeus, 1758: 419 (*)

ESPECIE TIPO: Meloe proscarabaeus Linnaeus, 1758, por designación subsecuente de Latreille (1810: 430).

Proscarabaeus Schrank, 1781: 225 (*)

ESPECIE TIPO: Meloe proscarabaeus Linnaeus, 1758, por tautonomía absoluta.

Melittophagus Kirby, 1818: 164

ESPECIE TIPO: Pediculus melittae Kirby, 1802 (= Meloe violaceus Marsham, 1802), por designación original (fide Selander, 1991; Bologna \& Pinto, 2002).

Triungulinus Dufour, 1828: 63 (*)

ESPECIE TIPO: Triungulinus andrenatarum Dufour, 1828, por monotipia.

Cnestocera Thomson, 1859: $124(*)$

ESPECIE TIPO: Meloe proscarabaeus Linnaeus, 1758, por designación original.

CONTENIDO Y DISTRIBUCIÓN: El subgénero está distribuido por gran parte de la región holártica (Pinto \& Bologna, 1999). En América del Norte se encuentran 18 especies (Pinto \& Bologna, 1999).

En México se ha citado la presencia de tres especies, M. dugesi, M. nebulosus y M. strigulosus. Además de estas especies, podrían encontrarse también, ya que se se han citado muy cerca de la frontera con los EEUU, las siguientes: Meloe (Meloe) franciscanus Van Dyke, 1928, Meloe (Meloe) niger Kirby, 1837, Meloe (Meloe) occultus Pinto \& Selander, 1970, Meloe (Meloe) vandykei Pinto et Selander, 1970. Por otro lado, Champion (1891) incluyó en la serie típica de $M$. dugesi dos ejemplares de Meloe (Meloe) tropicus Motschulsky, 1872, al parecer capturados en Xalapa. Pinto \& Selander (1970) indican 
que esa localidad es errónea y por lo tanto, la especie no se encontraría en México, aunque sí en Guatemala.

Meloe (Meloe) dugesi Champion, 1891

Meloe dugesi Champion, 1891: $366^{(*)}$

TERRA TYPICA: "Pinos Altos, Chihuahua" tras designación de lectotipo. Lectotipo designado por Pinto \& Selander (1970) en la colección del British Museum of Natural History (Londres).

Comentarios taxonómicos: Pinto \& Selander (1970) indican que la serie típica de la especie consta de 34 ejemplares, de los que hay al menos uno de cada una de las localidades siguientes: Córdoba, Durango, Xalapa y Toluca corresponden a $M$. nebulosus, otros dos de Xalapa corresponden a $M$. tropicus y un tercero a una especie no identificada.

DisTRIBUCIÓN GEOGRÁFICA: Especie distribuida por el norte de México que apenas penetra en el sur de los EEUU (Pinto \& Selander, 1970). En México se ha citado en los estados de: Chihuahua, Durango y Sonora. Pinto \& Selander (1970) precisan que la mayoría de las citas de Champion (1891) correspondientes a esta especie, recogidas también por Vaurie (1950), necesitan comprobación y no las consideran válidas, ya que la serie típica de $M$. dugesi incluía también ejemplares de $M$. nebulosus y de M. tropicus Motschulsky, 1856. Werner et al. (1966) citan M. dugesi en Arizona bajo la denominación de Meloe impressus Kirby, 1837, una especie que no se encuentra en México. Vaurie (1950) señala la presencia de $M$. duge$s i$ en Xalapa (Veracruz), pero la cita debe confirmarse.

MÉxICo: Chihuahua: Pinos Altos (Champion, 1891; Vaurie, 1950; Pinto \& Selander, 1970). Durango: Villa (Ciudad) Lerdo (Champion, 1891; Vaurie, 1950; Pinto \& Selander, 1970). Sonora: sin más precisión (Vaurie, 1950; Pinto \& Selander, 1970).

Meloe (Meloe) nebulosus Pinto et Selander, 1970

Meloe (Meloe) nebulosus Pinto et Selander, 1970: $154(*)$

TERRA TYPICA: "10 mi SE El Vergel, Chihuahua, México...".

Holotipo en la colección de la California Academy of Sciences (San Francisco, EEUU) (fide Pinto \& Selander, 1970).

COMENTARIOS TAXONÓMICOS: Ver comentarios con respecto a $M$. dugesi.

DisTRIBUCIÓN GEOGRÁFICA: Especie bien distribuida en México con algunas citas en Guatemala y en el extremo meridional de los EEUU (Pinto \& Selander, 1970). En México se ha citado en los estados de Chiapas, Chihuahua, Durango, Jalisco, México y Veracruz. Pinto \& Selander (1970) precisan que algunas de las citas de Champion (1891) de M. dugesi corresponden a esta especie. Además, estos autores sugieren que aunque no han examinado el ejemplar en cuestión, el registro de Amula (Guerrero) de Champion (1891) de M. dugesi también correspondería a $M$. nebulosus.

MÉXICo: Chiapas: Cordilleras de la vertiente del Pacífico (Pinto \& Selander, 1970). Chihuahua: 10 mi. al SE de El Vergel
(Pinto \& Selander, 1970). Durango: Victoria de Durango (Champion, 1891 sub M. dugesi; Pinto \& Selander, 1970); Sierra de Durango (Pinto \& Selander, 1970). Jalisco: Montañas al N de Ajijic (Pinto \& Selander, 1970). México: Ozumba de Alzate (Pinto \& Selander, 1970); Toluca (Champion, 1891 sub M. dugesi; Pinto \& Selander, 1970). Veracruz: Córdoba (Champion, 1891 sub M. dugesi; Pinto \& Selander, 1970); Xalapa (Champion, 1891 sub M. dugesi; Pinto \& Selander, 1970).

Meloe (Meloe) strigulosus Mannerheim, 1852

Meloe strigulosus Mannerheim, 1852: 349 (*)

TERRA TYPICA: "in insula Kodiak... marem in California boreali..." en la publicación original; "Calif. Boreali" tras designación de lectotipo (Pinto \& Selander, 1970). Lectotipo $\mathrm{n}^{\circ} 12549$ designado por Pinto \& Selander (1970) en la colección del Museum Zoologicum Universitatis (Helsinki).

COMENTARIOS TAXONÓMICOS: Especie bien caracterizada desde su descripción original.

DISTRIBUCIÓN GEOGRÁFICA: Especie repartida a lo largo de la costa del Pacífico desde la Península de Baja California hasta la Isla de Kodiak en Alaska, con citas en el Canadá y en los EEUU (Pinto \& Selander, 1970). En México sólo se ha citado en Baja California.

MÉxICo: Baja California: Sierra El Chinche (Horn, 1894 sub Meloe "allied to cordillerae").

Subgénero Treiodous Dugès, 1869

Treiodons Dugès, 1869a: $102(*)$

ESPECIE TIPO: Treiodons barranci Dugès, $1869(=$ M. laevis Leach, 1813), por monotipia.

Treiodous Dugès, 1869: Dugès, 1870: 169 (enmienda justificada) $\left(^{*}\right)$ Anchomeloe Iablokoff-Khnzorian, 1983a: 88 (*)

ESPECIE TIPO: Meloe autumnalis Olivier, 1792, por monotipia.

COMENTARIOS TAXONÓMICOS: Cros (1937: 197) menciona por error el nombre Triodous.

CONTENIDO Y DISTRIBUCIÓN: El subgénero incluye 6 especies a nivel mundial, de las cuales 5 se encuentran en América del Norte (Pinto \& Bologna, 1999). La corrección del nombre por el propio Dugès (1870) estaría justificada según el artículo 33.3.1 del Código Internacional de Nomenclatura Zoológica (CINZ, 2000).

En México se ha citado la presencia de dos especies, M. gracilicornis y M. laevis. Además de estas especies, podría encontrarse también Meloe (Treiodous) barbarus LeConte, 1861, ya que se ha citado en California (EEUU), muy cerca de la frontera con México.

Meloe (Treiodous) gracilicornis Champion, 1891

Meloe gracilicornis Champion, 1891: 367 (*)

TeRra tyPICA: "Mexico, Omilteme in Guerrero 8000 feet..., Juquila..." originalmente; "Omilteme, Guerrero", tras la designación de lectotipo. Lectotipo, designado por Pinto \& Selander (1970) en la colección del British Museum of Natural History (Londres).

COMENTARIOS TAXONÓMICOS: Incluida en Treiodous por Champion (1891) de manera informal.

DisTRIBUCIÓN GEOGRÁFICA: Especie bien distribuida en México con algunas citas en Guatemala (Pinto \& 
Selander, 1970). En México se ha citado en los estados de Chiapas, Guerrero, Hidalgo, México, Nayarit, Oaxaca y Puebla. Según Pinto \& Selander (1970) algunas de las citas de Champion (1891) y las de México de Van Dyke (1928) de M. laevis corresponden a esta especie.

MÉxICO: Chiapas: Rancho Nuevo, 6 mi. al SE de San Cristóbal de las Casas (Pinto \& Selander, 1970); San Cristóbal de las Casas (Pinto \& Selander, 1970); $8.5 \mathrm{mi}$. al S de San Cristóbal de las Casas (Pinto \& Selander, 1970); San Juan de Chamula, Bahom (Pinto \& Selander, 1970). Guerrero: Omilteme (Champion, 1891; Vaurie, 1950 sub M. laevis; Pinto \& Selander, 1970). Hidalgo: Zempoala (Pinto \& Selander, 1970). México: Atlacomulco (Pinto \& Selander, 1970); Toluca (Pinto \& Selander, 1970). Nayarit: Islas Tres Marías (Van Dyke, 1928 sub M. laevis; Vaurie, 1950 sub M. laevis; Pinto \& Selander, 1970). Oaxaca: Capulalpan (Champion, 1891 sub M. laevis; Vaurie, 1950 sub M. laevis; Pinto \& Selander, 1970); Juquila (Champion, 1891; Pinto \& Selander, 1970); La Parada (Champion, 1891 sub M. laevis; Vaurie, 1950 sub M. laevis; Pinto \& Selander, 1970); Las Peras (Champion, 1891 sub $M$. laevis; Vaurie, 1950 sub M. laevis; Pinto \& Selander, 1970); Mitla (Pinto \& Selander, 1970). Puebla: $6 \mathrm{mi}$. al O de Teziutlán (Pinto \& Selander, 1970).

\section{Meloe (Treiodous) laevis Leach, 1815}

Meloe laevis Leach, 1815: 249 (*)

TERRA TYPICA: "Insula Americes St. Domingo". Se conservan dos sintipos en la colección del British Museum of Natural History (Londres) (fide Pinto \& Selander, 1970).

Meloe cordillerae Chevrolat, 1844: 133

TERRA TYPICA: "Sainte Croix, près d'Orixaba" (fide Pinto \& Selander, 1970). Holotipo posiblemente en la colección del Muséum National d'Histoire Naturelle (París) (fide Pinto \& Selander, 1970). Meloe sublaevis LeConte, 1854c: 84 (*)

TERRA TYPICA: "Fort Union, New Mexico". Holotipo no 4950 en la colección del Museum of Comparative Zoology (Harvard University, Cambridge, EEUU) (fide Pinto \& Selander, 1970).

Meloe tridentatus Jiménez, 1866a: 230 (*)

TERRA TYPICA: "cerca del mineral del Chico y de Pachuca, en los sembrados de maíz de Atotonilco El Grande" (en Peñafiel Barranco, 1866 sub Meloe tuccia). La descripción de este taxón se realizó a partir de láminas presentadas por Peñafiel Barranco junto con su trabajo publicado en 1866

Treiodons barranci Dugès, 1869a: 102 (*)

TERRA TYPICA: "Silao,... Estado de Guanajuato...; Atotonilco el Grande, distrito del Estado de Hidalgo". Sintipos perdidos según Pinto \& Selander (1970). Vázquez \& Zaragoza (1979) indican como tipo el ejemplar $n^{\circ} 783$ (IBUNAM 002) de la colección del Instituto de Biología de la Universidad Nacional Autónoma de México.

Meloe opaca Motschulsky, 1872: 48 (non LeConte, 1861) (*)

TERRA TYPICA: “...des mêmes tropiques..." refiriéndose a Nicaragua, localidad que menciona en la descripción anterior de M. sculpticornis Motschulsky, 1872. Holotipo posiblemente en la colección del Museo de Zoología de la Universidad Estatal M. V. Lomonosov de Moscú (fide Pinto \& Selander, 1970).

Treiodous cordillerae (Chevrolat, 1844): Dugès, 1886a: 582 (*)

Treiodous barranci (Dugès, 1869): Dugès, 1889d: 35 (*)

Meloe laevis var. colombianus Beauregard, 1890: 480 (*)

TERRA TYPICA: no indicada [Colombia]. El holotipo se encontraría en la colección del Museo de Bruselas bajo el nombre de $M$. colombianus Buquet (in litt.) (fide Beauregard, 1890).

Meloe motshulskyi Pliginsky, 1914: 256 (nombre de sustitución para Meloe opaca Motschulsky, 1872) (*)

Meloe motschulskyi Pliginsky, 1914: Pinto et Selander, 1970: 111 (enmienda) (*)
Meloe barranci (Dugès, 1869): Leng et Mutchler, 1914: 467 (*)

COMENTARIOS TAXONÓMICOS: Beauregard (1890) y Borchmann (1917) trataron a $M$. sublaevis como especie independiente. Van Dyke (1928) incluyó en la sinonimia de esta especie el nombre no disponible M. montanus Chevrolat, 1837 (non LeConte, 1866).

DisTRIBUCIÓN GEOGRÁFICA: Su distribución incluye una amplia región desde Venezuela (Arnold, 1976) hasta el centro de los EEUU, con citas en Panamá, Colombia, Costa Rica, Haití, Honduras, Guatemala, México, Nicaragua y República Dominicana (Pinto \& Selander, 1970; Maes \& Huether, 2007). En México se ha encontrado en casi todos los estados, incluidos: Aguascalientes, Chiapas, Chihuahua, Coahuila, Distrito Federal, Durango, Guanajuato, Guerrero, Hidalgo, Jalisco, México, Michoacán, Morelos, Nuevo León, Oaxaca, Puebla, Querétaro, San Luis Potosí, Sinaloa, Sonora, Veracruz y Zacatecas. Varias de las citas de Champion (1892) y de Vaurie (1950) de esta especie corresponden a M. gracilicornis. MacSwain (1956) señala que la cita de Cros (1937) de $M$. tropicus en Guadalajara corresponde a $M$. laevis. Dugès (1889d) indica que las citas de M. tuccia? de Peñafiel Barranco (1866) (M. tucci según Dugès) corresponden a $M$. cordillerae.

MÉxICo: Aguascalientes: El Retorno, $10 \mathrm{mi}$ al E de Aguascalientes (Pinto \& Selander, 1970). Chiapas: Berriozábal (Pinto \& Selander, 1970); Cerro del Sumidero (Pinto \& Selander, 1970); Carretera al Sumidero, cerca de Tuxtla Gutiérrez (Pinto \& Selander, 1970); $3 \mathrm{mi}$. al O de Ocozocoautla (Pinto \& Selander, 1970); San Cristóbal de las Casas (Pinto \& Selander, 1970); Simojovel (Pinto \& Selander, 1970); Soyalo (Linsley et al., 1980); 5 mi. NNO de Tuxtla Gutiérrez (Pinto \& Selander, 1970). Chihuahua: San Pablo Balleza (Vaurie, 1950; Pinto \& Selander, 1970); Colonia Dublán (Pinto \& Selander, 1970); 10 mi. al S de Hidalgo del Parral (Pinto \& Selander, 1970); San José Bavícora (Vaurie, 1950; Pinto \& Selander, 1970); Santa Bárbara (Vaurie, 1950; Pinto \& Selander, 1970). Coahuila: $20 \mathrm{mi}$. al SE de Saltillo (Pinto \& Selander, 1970); Saltillo (Champion, 1891; Vaurie, 1950); Monclova (Champion, 1891; Vaurie, 1950). Distrito Federal: Atzcapotzalco (Pinto \& Selander, 1970); Ciudad de México (Dugès, 1889d sub T. cordillerae; Champion, 1891; Vaurie, 1950; Pinto \& Selander, 1970); Tlalpan (Pinto \& Selander, 1970); Valle de México (Peñafiel Barranco, $1866 \mathrm{sub}$ M. tuccia?). Durango: Durango (Quintero \& Canales, 1987); 8 mi. al NE de Victoria de Durango (Pinto \& Selander, 1970); 12 mi. al SO de Victoria de Durango (Pinto \& Selander, 1970); 5 mi. al O de Victoria de Durango (Pinto \& Selander, 1970); Victoria de Durango (Pinto \& Selander, 1970); El Saltito (Pinto \& Selander, 1970); Palos Colorados (Vaurie, 1950; Pinto \& Selander, 1970); San Lucas (Diez de Octubre) (Vaurie, 1950; Pinto \& Selander, 1970); San Juan del Río (Vaurie, 1950; Pinto \& Selander, 1970); Villa Madero (Vaurie, 1950; Pinto \& Selander, 1970); Ciudad Durango (Champion, 1891; Vaurie, 1950). Guanajuato: Guanajuato (Champion, 1891; Vaurie, 1950); Silao (Dugès, 1869a sub T. barranci; Champion, 1891; Vaurie, 1950; Pinto \& Selander, 1970; Vázquez \& Zaragoza, 1979). Guerrero: Amula (Champion, 1891; Vaurie, 1950); 
Xautipa (Champion, 1891; Vaurie, 1950). Hidalgo: Atotonilco el Grande (Peñafiel Barranco, 1866 sub M. tuccia?; Dugès, 1869a sub T. barranci); Molino de Guerrero (Pinto \& Selander, 1970); Pachuca (Peñafiel Barranco, 1866 sub M. tuccia?; Pinto \& Selander, 1970); Mineral del Chico (Peñafiel Barranco, $1866 \mathrm{sub}$ M. tuccia?); Río Metztitlán, 20 mi. al N de Atotonilco el Grande (Selander, 1954b; Pinto \& Selander, 1970); Atotonilco el Grande (Dugès, 1869a; Champion, 1891; Vaurie, 1950); San Miguel (Pinto \& Selander, 1970). Jalisco: El Refugio, 9 mi. al O de Tepatitlán (Pinto \& Selander, 1970); alrededores de Guadalajara (Cros, 1937 sub M. tropicus; Vaurie, 1950; MacSwain, 1956; Pinto \& Selander, 1970); Ocotlán (Pinto \& Selander, 1970); Tonalá (Pinto \& Selander, 1970). México: Amecameca (Pinto \& Selander, 1970); Chapingo (Pinto \& Selander, 1970); Ixtapan de la Sal (Pinto \& Selander, 1970); Real de Arriba (Pinto \& Selander, 1970); Tejupilco de Hidalgo (Pinto \& Selander, 1970); Teotihuacán (Vaurie, 1950; Pinto \& Selander, 1970); Texcoco (Pinto \& Selander, 1970); Toluca (Dugès, 1889d sub T. cordillerae; Champion, 1891; Vaurie, 1950; Pinto \& Selander, 1970). Michoacán: Morelia (Champion, 1891); $24 \mathrm{~km}$ al OSO del Cerro Tancítaro (Pinto \& Selander, 1970); $4 \mathrm{mi}$. al O de Quiroga (Pinto \& Selander, 1970); Tancítaro (Pinto \& Selander, 1970). Morelos: Cuernavaca (Champion, 1891; Vaurie, 1950; Pinto \& Selander, 1970); $4 \mathrm{mi}$. al NO de Cuernavaca (Pinto \& Selander, 1970); Santiago (Pinto \& Selander, 1970); 7.3 mi. al SSO de Yautepec (Pinto \& Selander, 1970). Nayarit: Islas Tres Marías (Vaurie, 1950). Nuevo León: Galeana (Pinto \& Selander, 1970). Oaxaca: Ruinas de Monte Albán (Pinto \& Selander, 1970); Oaxaca de Juárez (Champion, 1891; Pinto \& Selander, 1970); $16 \mathrm{mi}$. al SE de Oaxaca de Juárez (Pinto \& Selander, 1970); Tlacolula (Pinto \& Selander, 1970). Puebla: $5 \mathrm{mi}$. al S de Acatzingo (Pinto \& Selander, 1970); Necaxa (Pinto \& Selander, 1970); Puebla (Champion, 1891). Querétaro: cerca de Hacienda Balvanera (Pinto \& Selander, 1970); Querétaro (Pinto \& Selander, 1970). San Luis Potosí: San Luis Potosí (Champion, 1891; Vaurie, 1950; Pinto \& Selander, 1970). Sinaloa: Culiacán (Pinto \& Selander, 1970). Sonora: sin precisar (Champion, 1891; Vaurie, 1950). Veracruz: Córdoba (Pinto \& Selander, 1970); Xalapa (Champion, 1891; Vaurie, 1950; Pinto \& Selander, 1970); Orizaba (Champion, 1891; Vaurie, 1950; Pinto \& Selander, 1970); San Juan de la Punta, Coitláhuac (Pinto \& Selander, 1970); Sontecomapan (Champion, 1891; Vaurie, 1950; Pinto \& Selander, 1970); Tecamalucan (Pinto \& Selander, 1970); Xico (Pinto \& Selander, 1970); Veracruz (Dugès, 1889d sub T. cordillerae; Champion, 1891; Vaurie, 1950). Zacatecas: Fresnillo (Pinto \& Selander, 1970); Guadalupe (Vaurie, 1950; Pinto \& Selander, 1970); Zacatecas (Vaurie, 1950; Pinto \& Selander, 1970); 13 mi. al NO de Zacatecas (Pinto \& Selander, 1970).

\section{Género Spastonyx Selander, 1954}

Spastonyx Selander, 1954a: 13 (como subgénero de Calospasta) (*)

ESPECIE TIPO: Calospasta nemognathoides Horn, 1870, por designación original.

CONTENIDO Y DISTRIBUCIÓN: El género está representado por dos especies, S. nemognathoides (Horn, 1870) y S. macswaini (Selander, 1954), del suroeste de los Estados Unidos (Selander, 1985b). Werner et al. (1966) señalan la presencia de ambas especies en Yuma County (Arizona) y Pinto (1974b) indica la presencia de $S$. macswaini unas 12 mi. al $\mathrm{O}$ de Calexico, en California (EEUU), ambos registros localizados muy cerca de la frontera con México. Pinto \& Bologna (1999) citan la presencia del género Spastonyx en México, pero no indican ni especie ni localidad concreta.

Tribu Pyrotini MacSwain, 1956

Pyrotini MacSwain, 1956: $67(*)$

GÉNERo TIPO: Pyrota Dejean, 1834

CONTENIDO Y DISTRIBUCIÓN: La tribu, exclusiva de América, consta de unas 100 especies distribuidas en 10 géneros, la mayoría de ellos con una distribución limitada a América del Sur (Pinto \& Bologna, 1999). En México existen representantes de los géneros Denierota y Pyrota.

Género Denierota Kaszab, 1959

Denierota Kaszab, 1959: 105 (*)

ESPECIE TIPO: Lytta kraatzi Haag-Rutenberg, 1880, por designación original.

Paniculolytta Selander, 1960b: 80 (*)

ESPECIE TIPO: Lytta sanguineoguttata Haag-Rutenberg, 1880, por designación original.

CONTENIDO Y DISTRIBUCIÓN: El género está representado por dos especies Denierota kraatzi (Haag-Rutenberg, 1880) y Denierota sanguineoguttata (Haag-Rutenberg, 1880), distribuidas en Guatemala y El Salvador. Kaszab (1963a: 335) sinonimizó Paniculolytta con Denierota. Pinto \& Bologna (1999) señalan la presencia del género Denierota en el sur de México, aunque sin indicar ni especie ni localidad precisa.

\section{Género Pyrota Dejean, 1834}

Pyrota Dejean, 1834: 224

ESPECIE TIPO: Tetraonyx dispar Germar, 1824, por designación subsecuente de Aksentjev (1988: 578 sub Lytta dispar Germar, 1824). Germar (1824) cambió la asignación génerica de T. dispar a Lytta en la corrigenda del trabajo (Selander, 1991).

Cantharida Herrera et Mendoza, 1866: 265 (lapsus pro Cantharis) (*)

CONTENIDO Y DISTRIBUCIÓN: El género está representado por 42 especies distribuidas a lo largo del continente americano (Pinto \& Bologna, 1999). Desde los trabajos de Denier (1934), el género no ha vuelto a ser revisado adecuadamente y únicamente se han definido algunos grupos de especies de América del Norte (Selander, 1954b; 1982b; 1983b,c,d; Pinto \& Bologna, 1999). Selander (1964a) señala que en América del Norte se encuentran 31 especies. En México se han citado 24 especies y además, es posible que también se encuentre Pyrota perversa Dillon, 1952, ya que se ha colectado en Texas (EEUU) cerca de la frontera (Selander, 1982b).

\section{Pyrota akhurstiana Horn, 1891}

Pyrota akhurstiana Horn, 1891: 44 (*)

Terra tYPICA: “N. Mexico... and at Tucson, Ariz. ...”. Tipos posiblemente en la colección del Museum of Comparative Zoology de Harvard (EEUU). 
Cantharis akhurstiana (Horn, 1891): Champion, 1899: 171 (*)

DISTRIBUCIÓN GEOGRÁFICA: Especie distribuida por el norte de México y sur de los EEUU (Champion, 1892; Vaurie, 1950). En México se ha citado de Chihuahua, Sinaloa y Sonora.

MÉXICo: Chihuahua: Entre Camargo y Parral (Spieth, 1950); 20 mi. al SO de Camargo (Vaurie, 1950); 42 mi. al SO de Camargo (Vaurie, 1950); 20 mi. al S de Ciudad Juárez (Selander, 1954b); Chihuahua (Selander, 1964a); 7 mi. al N de Chihuahua (Selander, 1954b); Colonia Dublán (Selander, 1954b); Delicias (Vaurie, 1950); $10 \mathrm{mi}$. al S de Delicias (Vaurie, 1950); Río San Pedro, $49 \mathrm{mi}$. al S de Chihuahua (Selander, 1954b); Santa Bárbara (Vaurie, 1950); 63 mi. al O de Santa Bárbara (Vaurie, 1950); Valle de Olivos (Vaurie, 1950). Sinaloa: Los Mochis (Werner et al., 1966). Sonora: Ciudad Obregón (Werner et al., 1966); Hermosillo (Werner et al., 1966); Navajoa (Werner et al., 1966).

\section{Pyrota bilineata Horn, 1885}

Pyrota bilineata Horn, 1885: 115 (*)

TERRA TYPICA: “...Colorado and Arizona”. Tipos posiblemente en la colección del Museum of Comparative Zoology de Harvard (EEUU).

Cantharis bilineata (Horn, 1885): Champion, 1899: 173 (*)

DISTRIBUCIÓN GEOGRÁFICA: Especie distribuida por el norte de México y sur de los EEUU (Selander, 1954b, 1964a). En México se ha citado de Chihuahua, Durango y Sonora.

MÉxICo: Chihuahua: 44 mi. al S de Ciudad Juárez (Selander, 1954b). Durango: norte de Durango (Werner et al., 1966). Sonora: Norte de Sonora (Champion, 1892; Selander, 1954b).

\section{Pyrota concinna Casey, 1891}

Pyrota concinna Casey, 1891: 174 (*)

TERRA TYPICA: "Western Texas". Lectotipo designado por Selander (1982b) en la colección del United States National Museum of Natural History (Washington D.C.).

Cantharis concinna (Casey, 1891): Champion, 1899: 175 (*)

COMENTARIos TAXONÓMICOS: Champion (1892) incluyó a $P$. concinna en la sinonimia de $P$. terminata, y Borchmann (1917) la trató como variedad de $P$. terminata. Denier (1934), seguido por Selander (1982b), la restituye a nivel específico y señala que la especie se ha venido citando por diversos autores con los nombres de $P$. mylabrina, $P$. terminata y $P$. palpalis. Selander (1982b) la incluye en el grupo de $P$. mylabrina.

DISTRIBUCIÓN GEOGRÁFICA: Se encuentra en el norte de México y sur de los EEUU (Selander, 1964a). En México se ha citado en los estados de Chihuahua, Sonora y Zacatecas. Selander (1982b) indica que parte de las citas de Vaurie (1950) de P. mylabrina en México corresponden en realidad a $P$. concinna.

MÉxICo: Chihuahua: 8 mi. al SE de Ciudad Camargo (Selander, 1982b); Chihuahua (Selander, 1982b); 13 mi. al S de Ciudad Juárez (Selander, 1982b); Delicias (Vaurie, $1950 \mathrm{sub} P$. mylabrina; Selander, 1982b); $18 \mathrm{mi}$. al O de Jiménez
(Selander, 1982b); Salaices (Selander, 1982b); Km. 36 entre Santa Bárbara y Ojito (Selander, 1982b); Manantial de Santa Rosalía, cerca de Ciudad Camargo (Selander, 1982b). Sonora: 38 mi. al Oeste de Sonoyta (Selander, 1958). Zacatecas: Fresnillo (Vaurie, 1950 sub P. mylabrina; Selander, 1982b).

Pyrota deceptiva Selander, 1963

Pyrota deceptiva Selander, 1963: 33 (*)

Terra typica: "San Antonio, Loop 13, Texas". Holotipo en la colección del Essig Museum of Entomology (University of California at Berkeley, EEUU) (fide Selander, 1963, 1982b).

Comentarios taXonómicos: Selander (1982b) la incluye en el grupo de P. mylabrina.

DISTRIBUCIÓN GEOGRÁFICA: Especie distribuida por el noreste de México y sureste de los EEUU (Selander, 1982b). En México se ha citado en Nuevo León. Selander (1963b, 1982b) indica que esta especie se ha venido citando en Monterrey bajo los nombres de $P$. terminata y $P$. palpalis por varios autores (Champion, 1892; Denier, 1934; Vaurie, 1950).

MÉxICo: Nuevo León: Apodaca (Selander, 1963, 1982b); El Diente, cerca de Monterrey (Selander, 1963, 1982b); 14 mi. al N de Linares (Selander, 1963, 1982b); Monterrey (Selander, 1963, 1982b).

Pyrota decorata (Haag-Rutenberg, 1880)

Lytta decorata Haag-Rutenberg, 1880: 51 (*)

TERRA TYPICA: "Guatimala (sic)". Posiblemente en la colección Haag-Rutenberg del Zoologische Staatsammlung de Munich.

Pyrota decorata (Haag-Rutenberg, 1880): Beauregard, 1890: 515 (*)

Cantharis decorata (Haag-Rutenberg, 1880): Champion, 1899: 176 (*)

Pyrota decorata var. semicinctipennis Pic, 1916: 10 (*)

TERRA TYPICA: "Guatemala"

DistribuCIÓN GEOGRÁFICA: Especie distribuida desde el sur de México hasta Panamá (Champion, 1892; Borchmann, 1917; Maes \& Huether, 2007). En México se ha citado en los estados de Oaxaca y Veracruz.

MÉXICo: Oaxaca: Tehuantepec (Champion, 1892; Denier, 1934). Veracruz: Xalapa (Champion, 1892; Denier, 1934); Plan del Río (Champion, 1892).

Pyrota divirgata (Villada et Peñafiel, 1867)

Cantharis divirgata Villada et Peñafiel, in Herrera et Mendoza, 1867: $14(*)$

TERRA TYPICA: “...entre Yautepec y Cuernavaca...”. Tipo en paradero desconocido.

Lytta clavipalpis Haag-Rutenberg, 1880: $50(*)$

TERRA TYPICA: "Mexico". Tipo posiblemente en la colección HaagRutenberg del Zoologische Staatsammlung de Munich.

Pyrota clavipalpis (Haag-Rutenberg, 1880): Beauregard, 1890: 515 (*) Pyrota divirgata (Villada \& Peñafiel, 1867): Champion, 1892: 433 (*)

COMENTARIOS TAXONÓMICOS: Berg (1883) incluyó a $C$. maculata en la sinonimia de $P$. divirgata, una opinión no seguida por autores recientes. Por otra parte, Selander (1963) restablece el estatus específico para L. nigrovittata, especie que Champion (1892), seguido por Borchmann (1917), Denier (1934) y Selander (1957b), había incluido en la sinonimia de $C$. divirgata. Champion (1892) y 
Borchmann (1917) tratan a P. clavipalpis como variedad de $P$. divirgata, donde se incluye en la actualidad.

DISTRIBUCIÓN GEOGRÁFICA: Especie distribuida por el norte de México y sureste de los EEUU (Selander, 1964a). En México se ha citado de Chiapas, Guerrero, Morelos, Oaxaca y Puebla (Selander, 1954b, 1964a). La cita de Veracruz de Champion (1892) coresponde a $P$. nigrovittata. Existe una cita dudosa de Nicaragua (Maes, 1989; Maes \& Huether, 2007).

MÉxICo: Chiapas: (Selander, 1954b). Guerrero: Chilpancingo (Champion, 1892, 1893; Selander, 1964a); Mexcala (Selander, 1954b); 5 mi. al N de Mexcala (Selander, 1954b); Mochitlán (Champion, 1892). Morelos: entre Yautepec y Cuernavaca (Villada \& Peñafiel, 1867; Dugès, 1870; Champion, 1892); Yautepec (Maes \& Huether, 2007). Oaxaca: Oaxaca (Champion, 1892). Puebla: Atlixco (Champion, 1892); Izúcar de Matamoros (Champion, 1893).

Pyrota fasciata Selander, 1963

Pyrota fasciata Selander, 1963: 36 (*)

TeRra tYPICA: "Quemado, Texas". Holotipo en la colección del

Field Museum of Natural History (Chicago, EEUU) (fide Selander, 1963, 1982b).

COMENTARIOS TAXONÓMICOS: Selander (1982b) la incluye en el grupo de $P$. mylabrina.

DisTRIBUCIÓN GEOGRÁFICA: Especie distribuida por el noreste de México y sureste de los EEUU (Selander, 1982b). En México se ha citado en Coahuila y Nuevo León.

MÉxICo: Coahuila: $12 \mathrm{mi}$. al N de Hermanas (Selander, 1982b). Nuevo León: Apodaca (Selander, 1963, 1982b); Pesquería (Selander, 1963, 1982b); Sabinas Hidalgo (Selander, 1963, 1982b); Vallecillo (Selander, 1963, 1982b); Villa de Santiago (Selander, 1963, 1982b).

\section{Pyrota hirticollis Champion, 1892}

Pyrota hirticollis Champion, 1892: 435 (*)

TERRA TYPICA: "MeXICO, Acapulco". Lectotipo, designado por Selander (1983d) en la colección del British Museum of Natural History (Londres). La serie típica consta de tres ejemplares (fide Selander, 1983d).

Pyrota tenuicostata? var. hirticollis Champion, 1892: Denier, 1935a: $172(*)$

Cantharis hirticollis (Champion, 1892): Champion, 1899: $179(*)$

COMEnTARIOS TAXONÓMICOs: Denier (1935a) y Blackwelder (1945) incluyen a $P$. hirticollis como posible variedad de $P$. tenuicostatis, pero Selander (1983d) mantiene su estatus específico. Selander (1982b) incluye a $P$. hirticollis en el grupo de $P$. tenuicostatis.

DistribuCIÓN GEOGRÁFICA: Especie endémica de México (Selander, 1983d), aunque existen registros que requieren confirmación en Nicaragua (Maes \& Huether, 2007), citada en los estados de Guerrero, Nayarit, Puebla, Sinaloa y Sonora.

MÉXICo: Guerrero: Acapulco (Champion, 1892; Denier, 1934; Selander, 1983d); Chilpancingo (Selander, 1983d). Nayarit: 24 mi. al N de Acaponeta (Selander, 1983d); San Blas
(Selander, 1983d). Puebla: Acatlán (Selander, 1983d). Sinaloa: $18 \mathrm{mi}$. al N de Culiacán (Selander, 1983d); 8 mi. al S de Elota (Selander, 1983d); 5 mi. al N de Mazatlán (Selander, 1983d); Mazatlán (Selander, 1983d); El Mármol, $40 \mathrm{~km}$ al N de Mazatlán (Selander, 1983d); 21 mi. al E de Villa Unión (Selander, 1983d); Venodia (Venedillo) (Selander, 1983d). Sonora: Álamos (Selander, 1983d); Arroyo del Juchijaqui, Álamos (Selander, 1983d); Hermosillo (Selander, 1983d).

Pyrota insulata (LeConte, 1858)

Lytta insulata LeConte, 1858a: 22 (*)

TERRA TYPICA: “...on the route to Llano Estacado". Holotipo $\mathrm{n}^{\mathrm{o}}$ 5104, en la colección del Museum of Comparative Zoology (Harvard University, Cambridge, EEUU) (fide Selander, 1982b).

Pyrota insulata (LeConte, 1858): LeConte, 1866: 159 (*)

Pyrota terminata LeConte, 1866: 159 (*)

TERRA TYPICA: "Kansas, near the Rocky Mountains". Holotipo ${ }^{\circ}$ 5101, en la colección del Museum of Comparative Zoology (Harvard University, Cambridge, EEUU) (fide Selander, 1982b).

COMEnTARIOS TAXONÓmicos: Champion (1892) incluyó en la sinonimia de $P$. terminata a $P$. concinna, una especie válida en la actualidad. Selander (1982b) establece la sinonimia de $P$. terminata con $P$. insulata. Selander (1982b) la incluyó en el grupo de P. mylabrina.

DISTRIBUCIÓN GEOGRÁFICA: Especie distribuida por el noreste de México y el centro y este de los EEUU (Selander, 1982b). Champion (1892) indica que las citas de P. mylabrina de LeConte (1853) y las de Horn (1885) corresponden a $P$. terminata. En México se ha citado de Coahuila, Durango, Nuevo León y Tamaulipas. Según Selander (1982b) $P$. insulata ha sido citada por diversos autores como $P$. engelmanni LeConte, 1847, una especie que no se encuentra en México. Las citas originales de $P$. terminata en México de Champion (1892) corresponden a P. palpalis, tal como el propio Champion (1893: 462) indicó.

MÉXICo: Coahuila: $10 \mathrm{mi}$. al O de Sabinas (Selander, 1982b). Durango: Ciudad Lerdo (Champion, 1892; Denier, 1934; Vaurie, 1950; Selander, 1982b). Nuevo León: Apodaca (Selander, 1982b); Monterrey (Selander, 1982b); Villa de Santiago (Selander, 1982b). Tamaulipas: Soto La Marina (Selander, 1982b).

Pyrota mariarum Champion, 1892

Pyrota mariarum Champion, 1892: 435 (*)

TeRRA TYPICA: "MeXICO, Tres Marías Is.". Lectotipo, designado por Selander (1983d) en la colección del British Museum of Natural History (Londres). La serie típica consta de tres ejemplares (fide Selander, 1983d).

Pyrota tenuicostata? var. mariarum Champion, 1892: Denier, 1935a: $172(*)$

Cantharis mariarum (Champion, 1892): Champion, 1899: $182(*)$

COMENTARIOS TAXONÓMICOS: Denier (1935a) y Blackwelder (1945) incluyen a P. mariarum como posible variedad de $P$. tenuicostatis, pero Selander (1983d) mantiene su estatus específico. Champion (1892) señala que es muy similar a $P$. hirticollis. Selander (1982b) incluye a $P$. mariarum en el grupo de $P$. tenuicostatis. 
DisTRIBUCiÓN GEOGRÁFICA: Especie endémica de México (Selander, 1983d), sólo conocida de las Islas Tres Marías.

MÉXICO: Nayarit: Islas Tres Marías (Champion, 1892; Denier, 1934; Selander, 1983d).

Pyrota mylabrina Chevrolat, 1834

Pyrota mylabrina Chevrolat, 1834a: fasc. $3, \mathrm{n}^{\circ} 53$ (sin paginar) (*)

TeRra tyPica: "Tuspan". Lectotipo, designado por Selander (1982b) en la colección del British Museum of Natural History (Londres).

Lytta mylabrina (Chevrolat, 1834): LeConte, 1853: 337 (y error de identificación) $(*)$

Cantharis milabrina (Chevrolat, 1834): Dugès, 1869b: 125 (y error tipográfico) (*)

COMENTARIOS TAXONÓMICOS: Beauregard (1890) incluye en la sinonimia de $P$. mylabrina el nombre no disponible Pyrota quadripunctata Klug in Dejean, 1837: 246. Selander (1982b) incluye a esta especie en el grupo de $P$. mylabrina.

DisTRIBUCIÓN GEOGRÁFICA: Especie distribuida por el norte y este de México y el extremo sureste de los EEUU (Selander, 1982b). Según Champion (1892) las citas de LeConte (1853) y Horn (1885) corresponden a P. terminata. En México se ha citado de Coahuila, San Luis Potosí y Veracruz. De acuerdo con Selander (1954b; 1982b) las citas de P. mylabrina en México de Dugès (1889e) y la mayor parte de las de Vaurie (1950) corresponden a $P$. palpalis o a $P$. concinna.

MÉxICo: Coahuila: Boquillas del Carmen (Selander, 1982b); San Pedro de Colonias (Champion, 1892; Vaurie, 1950; Selander, 1982b). San Luis Potosí: El Salto (Selander, 1982b). Veracruz: San Andrés Tuxtla (Selander, 1964a); Tuxpan (Chevrolat, 1834a; Dugès, 1869b; Champion, 1892; Denier, 1934; Vaurie, 1950; Selander, 1982b).

Pyrota nigrovittata (Haag-Rutenberg, 1880)

Lytta nigrovittata Haag-Rutenberg, 1880: 51 (*)

TeRRA tYPICA: "Mexico, Mirador". Lectotipo designado por Selander (1963) en la colección del Zoologische Staatssammlung (Munich).

Pyrota nigrovittata (Haag-Rutenberg, 1880): Beauregard, 1890: 516 (*) Lytta (Pyrota) nigrovittata var. adolphi Pic, 1916: 10 (*)

TERRA TYPICA: "Mexique". El tipo está en la colección del Muséum National d'Histoire Naturelle (Paris) (fide Selander, 1963).

Pyrota bicurvata Selander, 1957b: 135 (*)

TERRA TYPICA: "[Ciudad] Victoria, Tamaulipas...". Holotipo en la colección del American Museum of Natural History (EEUU) (fide Selander, 1957b)

COMENTARIOS TAXONÓMICOS: Selander (1963) restablece el estatus específico de L. nigrovittata, especie que Champion (1892) había sinonimizado previamente con C. divirgata, confirma la sinonimia de la var. adolphi y además, establece la sinonimia de $P$. bicurvata que describió él mismo al pensar que la sinonimia de L. nigrovittata con C. divirgata era correcta. Selander (1957b) señala que esta especie ha sido confundida con $P$. tenuicostatis.
DISTRIBUCIÓN GEOGRÁFICA: Especie distribuida por el norte de México y sureste de los EEUU (Selander, 1964a). En México se ha citado de Nuevo León, San Luis Potosí, Tamaulipas y Veracruz.

MÉxico: Nuevo León: Apodaca (Selander, 1964a); Linares (Selander, 1957b sub P. bicurvata); Monterrey (Selander, 1957b sub P. bicurvata); Vallecillo (Selander, 1957b sub P. bicurvata). San Luis Potosí: $11 \mathrm{~km}$ al E de Ciudad Valles (Selander, 1957b sub P. bicurvata). Tamaulipas: Abasolo (Selander, 1957b sub P. bicurvata); Ciudad Victoria (Selander, 1957b sub P. bicurvata). Veracruz: Hacienda El Mirador (Haag-Rutenberg, 1880; Champion 1892 sub P. divirgata; Selander, 1963); Plan del Río (Champion, 1892 sub $P$. divirgata).

Pyrota nobilis (Haag-Rutenberg, 1880)

Lytta nobilis Haag-Rutenberg, 1880: $48(*)$

TERRA TYPICA: "Mexico". Lectotipo designado por Selander (1983c) en la colección del Zoologische Staatsaammlung (Munich).

Cantharis nobilis (Haag-Rutenberg, 1880): Beauregard, 1890: 495 (*) Pyrota nobilis (Haag-Rutenberg, 1880): Champion, 1892: 436 (*)

COMENTARIOS TAXONÓMICOS: Especie incluida en el grupo de $P$. nobilis por Selander (1983c).

Distribución GEOGRÁFICA: Especie exclusiva de México (Vaurie, 1950; Selander, 1983c). En México se ha citado de Durango, Jalisco y Oaxaca. Según Selander (1983c) las citas más recientes de la especie datan de 1927.

MÉxICo: Durango: Canelas (Champion, 1892; Denier, 1934; Vaurie, 1950; Selander, 1983c). Jalisco: Tonila (Champion, 1892; Denier, 1934; Vaurie, 1950; Selander, 1983c). Oaxaca: Oaxaca (Champion, 1892; Vaurie, 1950).

\section{Pyrota pacifica Selander, 1983}

Pyrota pacifica Selander, 1983c: $462(*)$

TERRA TYPICA: "Esperanza, 1000m., Vulkan [Volcán] de Colima, [Colima or Jalisco,] Mexico...". Holotipo en la colección del Zoologische Staatsaammlung (Munich) (fide Selander, 1983c).

COMENTARIOS TAXONÓMICOS: Especie incluida en el grupo de $P$. pacifica por Selander (1983c).

DistRIBUCIÓN GEOGRÁFICA: Especie endémica de México (Selander, 1983c). En México se ha citado en Morelos y Colima-Jalisco.

MÉxICo: Colima-Jalisco: La Esperanza, Volcán de Colima (Selander, 1983c). Morelos: Cuernavaca (Selander, 1983c).

\section{Pyrota palpalis Champion, 1893}

Pyrota palpalis Champion, 1893: 462 (*)

Terra tYPICA: "MeXICO, Villa Lerdo in Durango, Chihuahua City, Monterey in Nuevo Leon..." en la publicación original; "Villa Lerdo, Durango" tras designación de lectotipo (fide Selander, 1982b). Lectotipo designado por Selander (1982b) en la colección del British Museum of Natural History (Londres).

Cantharis palpalis (Champion, 1893): Champion, 1899: $184\left({ }^{*}\right)$

COMENTARIOS TAXONÓMICOS: Selander (1982b) la incluye en el grupo de P. mylabrina.

Graellsia, 63(2), Diciembre 2007, pp. 165-258 - ISSN: 0367-5041 
DISTRIBUCIÓN GEOGRÁFICA: Especie distribuida por el norte de México y sur de los EEUU (Selander, 1964a; 1982b). En México se ha citado de los estados de Chihuahua, Coahuila, Durango, Nuevo León, Sonora y Zacatecas. Champion (1893) indica que todas sus citas anteriores de $P$. terminata (Champion, 1892) corresponden a $P$. palpalis. Selander (1982b) indica además que $P$. palpalis se ha citado en México como $P$. mylabrina por Dugès (1889e) y por Vaurie (1950).

MÉxICo: Chihuahua: $8 \mathrm{mi}$. al SE de Ciudad Camargo (Selander, 1982b); 11 mi. al S de Ciudad Camargo (Selander, 1982b); 13 mi. al S de Ciudad Juárez (Selander, 1982b); Chihuahua (Champion, 1892 sub P. terminata; Champion, 1893; Denier, 1934; Vaurie, 1950; Selander, 1982b); Delicias (Vaurie, 1950 sub P. mylabrina); $3 \mathrm{mi}$. al SE de Delicias (Selander, 1982b); 10 mi. al N de Jiménez (Selander, 1982b); 6 mi. al NE de Meoqui (Selander, 1982b); Ojo del Lucero (Selander, 1982b); 5 mi. al N de Ojo del Lucero (Selander, 1982b); Samaluyaca (Selander, 1982b); Río San Pedro, 39 mi. al S de Chihuahua (Selander, 1954b; Selander, 1982b); 15 mi. al S de Villa Ahumada (Selander, 1982b). Coahuila: 11 mi. al O de Saltillo (Selander, 1964a); La Rosa (Vaurie, 1950 sub $P$. mylabrina; Selander, 1982b); 5 mi. al N de Parras (Selander, 1982b); Saltillo (Selander, 1982b); 5 mi. al N de Saltillo (Vaurie, 1950 sub P. mylabrina); San Pedro de Colonias (Selander, 1982b). Durango: $60 \mathrm{mi}$. al N de Durango (Selander, 1982b); Gómez Palacio (Selander, 1982b); 1 mi. al N de Gómez Palacio (Selander, 1982b); 2 mi. al N de Menores de Arriba (Primo Verdad) (Selander, 1982b); Ciudad Durango (Selander, 1982b); Ciudad Lerdo (Dugès, 1889e sub P. mylabrina; Champion, 1892 sub P. terminata; Champion, 1893; Denier, 1934; Vaurie, 1950; Selander, 1982b). Nuevo León: Monterrey (Champion, 1892 sub P. terminata; Champion, 1893; Denier, 1934; Vaurie, 1950). Sonora: Desemboque (Selander, 1982b); Hermosillo (Selander, 1982b); San Francisco, carretera a La Salina (Selander, 1982b); 35 mi. al O de Sonoyta (Selander, 1982b); $80 \mathrm{mi}$. al NO de Sonoyta (Selander, 1982b).

Pyrota plagiata (Haag-Rutenberg, 1880)

Cantharis maculata Lacordaire, 1859 (Atlas): lám. 60, fig. 4 (non Villers, 1789) (*)

TERRA TYPICA: "Amér. bor"

Lytta plagiata Haag-Rutenberg, 1880: 49 (*)

TERRA TYPICA: "Mexico". Lectotipo designado en la colección del Zoologische Staatssammlung (Munich) (Selander, 1983b).

Cantharis lacordairei Berg, 1881: 303 (nombre substituto de C. maculata Lacordaire, 1859) (*)

Pyrota lacordairei (Berg, 1881): Beauregard, 1890: 516 (*)

Pyrota plagiata (Haag-Rutenberg, 1880): Beauregard, 1890: 516 (*)

Cantharis plagiata (Haag-Rutenberg, 1880): Champion, 1899: 185 (*)

COMENTARIOS TAXONÓMICOS: La historia taxonómica de esta especie es complicada, tanto por problemas nomenclaturales como por problemas de identificación. Lacordaire (1859: Atlas) publica una figura muy detallada (sin acompañar de una descripción) de una especie del género Pyrota de América septentrional con el nombre de Cantharis maculata Klug. Este taxón, por lo tanto, no corresponde a Lytta maculata Say, 1823, que es una especie del género Epicauta. Sin embargo, el nombre de Cantharis maculata ya fue utilizado por Villers (1789). En esta situación, el nombre de Haag-Rutenberg (1880) tendría validez. Además de esta cuestión nomenclatural, Champion (1892) trató a C. maculata y P. plagiata como sinónimos de $P$. postica, Burmeister (1881) trató erróneamente a $P$. vittigera Blanchard, 1843 (non Leconte, 1858) como sinónimo de $P$. maculata (ver comentarios de Berg, 1881) y el propio Berg (1883) incluye a $C$. maculata en la sinonimia de $P$. divirgata. Borchmann (1917) incluye a C. maculata, L. plagiata y C. lacordairei en la sinonimia de $P$. postica, mientras que Werner (1954a) y Selander (1983b) mantiene la validez de $P$. plagiata. Champion (1892) señala además la existencia de los nombres no disponibles Pyrota maculosa Klug in Dejean, 1837: 246 y Pyrota plagiata Sturm, 1843: 174.

Selander (1982b) la incluye en el grupo de P. postica.

DISTRIBUCIÓN GEOGRÁFICA: Especie distribuida por el centro y norte de México y el suroeste de los EEUU (Selander, 1983b). En México se ha citado de Chihuahua, Durango, Jalisco, Morelos, Nayarit, San Luis Potosí y Zacatecas. Esta especie se ha citado como $P$. divirgata por Denier (1934) y como $P$. postica por Champion (1892), Denier (1934), Spieth (1950) y Vaurie (1950). Según Selander (1983c), la cita de P. postica de Ahualulco (Jalisco) recogida por Dugès (1889e), Champion (1892) y Vaurie (1950) y transferida a $P$. plagiata por Werner (1954) corresponde en realidad a $P$. rugulipennis.

MÉxICo: Chihuahua: 8 mi. al N de Gallego (Selander, 1983b); 2 mi. al O de Pedernales (Selander, 1983b); Estación Ferrocarril Babícora (Spieth, 1950 sub P. postica); San José Babícora (Vaurie, 1950 sub P. postica; Werner, 1954a; Selander, 1983b); Santa Bárbara (Vaurie, 1950 sub P. postica; Selander, 1983b). Durango: $14 \mathrm{mi}$. al NE de Durango (Selander, 1983b); 66 mi. al S de Hidalgo del Parral (Selander, 1983b). Jalisco: Guadalajara (Selander, 1983b); 22 mi. al NO de La Piedad (Selander, 1983b); 10 mi. al NO de Magdalena (Selander, 1983b); Tequila (Selander, 1983b); $1 \mathrm{mi}$. al N de Tequila (Selander, 1983b). Morelos: Cuautla (Selander, 1983b); Cuernavaca (Selander, 1983b); Xochicalco (Selander, 1983b). Nayarit: Jesús María (Selander, 1983b); La Mesa de Nayar (Selander, 1983b). San Luis Potosí: 18 mi. al O de San Luis Potosí (Selander, 1983b); Tepetate (Selander, 1983b). Zacatecas: $1.5 \mathrm{mi}$. al O de Fresnillo (Selander, 1983b).

Pyrota postica LeConte, 1866

Pyrota postica LeConte, 1866: 160 (*)

TerRa tYPICA: "Texas and New Mexico" originalmente; "Texas" tras designación de lectotipo. Lectotipo $\mathrm{n}^{\circ} 5103$, designado por Selander (1983b) en la colección del Museum of Comparative Zoology (Harvard University, Cambridge, EEUU).

Comentarios tAXonómicos: Champion (1892) incluyó en la sinonimia de $P$. postica a $C$. maculata y a P. plagiata. Borchmann (1917), además, incluyó en la 
sinonimia de $P$. postica a $P$. lacordairei (ver sinonimia de P. maculata). Denier (1934) sigue a esos autores, pero Selander (1983b) incluye estas sinonimias en P. plagiata, que a su vez es un sinónimo de $P$. maculata.

Selander (1982b) incluye a esta especie en el grupo de $P$. postica.

DISTRIBUCIÓN GEOGRÁFICA: Especie distribuida por el norte de México y el sur de los EEUU (Selander, 1983b). Borchmann (1917) indica que las citas de P. mylabrina de LeConte $(1853,1866)$, Horn (1885) y parte de las de Dugès $(1889 \mathrm{e})$ corresponden a $P$. postica, aunque, como indica Selander (1983b), podrían corresponder en realidad a $P$. maculata, mientras que Champion (1892) atribuía algunas de éstas a $P$. terminata. Mientras no se revisen estos ejemplares, parece razonable no considerar las citas dudosas. En México se ha citado de Chihuahua, Coahuila, Durango y Nuevo León. Según Selander (1983c) la cita de $P$. postica de Ahualulco (Jalisco) de Dugès (1889e), repetida por Denier (1934), corresponde a $P$. rugulipennis y no a $P$. maculata ( $P$. plagiata) como indica Werner (1954).

MÉxICo: Chihuahua: Colonia Dublán (Selander, 1983b); Colonia Juárez (Selander, 1983b); $12 \mathrm{mi}$. al S de Villa Ahumada (Selander, 1983b); $15 \mathrm{mi}$. al S de Villa Ahumada (Selander, 1983b); Moctezuma (Selander, 1983b). Coahuila: Saltillo (Selander, 1983b). Durango: $19 \mathrm{mi}$. al N de Cuencamé (Selander, 1964a, 1983b); 7 mi. al SO de Cuencamé (Selander, 1983b); Tlahualillo de Zaragoza (Selander, 1983b); Ciudad Lerdo (Champion, 1892; Denier, 1934; Vaurie, 1950; Selander, 1983b). Nuevo León: Monterrey (Horn, 1885; Denier, 1934; Vaurie, 1950; Selander, 1983b).

Pyrota punctata Casey, 1891

Pyrota punctata Casey, 1891: 173 (*)

TeRrA TYPICA: "Western Texas". Lectotipo no 49211, designado por Selander (1982b) en la colección del USNM.

Cantharis punctata (Casey, 1891): Champion, 1899: 185 (*)

Comentarios taxonómicos: Selander (1982b) la incluye en el grupo de P. mylabrina.

DISTRIBUCIÓN GEOGRÁFICA: Especie distribuida por el norte de México y sur de los EEUU (Selander, 1982b). En México se conoce de Chihuahua, Coahuila y Durango. Según Selander (1982b), las citas de Oaxaca (Valerio Trujano) y de Hidalgo (Pachuca) del propio Selander (1954b), corresponden a errores de etiquetado.

MÉxICo: Chihuahua: 7 mi. al SE de Ciudad Juárez (Selander, 1982b); 20 mi. al S de Ciudad Juárez (Selander, 1954b; Selander, 1982b); 2 mi. al N de Jiménez (Selander, 1982b); 6 mi. al NE de Meoqui (Selander, 1982b); Samalayuca (Selander, 1982b). Coahuila: Buena Vista, Sierra del Carmen (Selander, 1982b); 5 mi. al N de Parras (Selander, 1982b); San Pedro de las Colonias (Champion, 1892; Denier, 1934; Vaurie, 1950; Selander, 1954b; Selander, 1982b); Torreón (Vaurie, 1950; Selander, 1982b). Durango: Gómez Palacio (Selander, 1982b); La Loma (Selander, 1982b); Tlahualillo de Zaragoza (Selander, 1982b); 10 mi. SO Ciudad Lerdo (Selander, 1964a; 1982b); Ciudad Lerdo (Champion, 1892; Denier, 1934; Vaurie, 1950; Selander, 1982b).

\section{Pyrota quadrinervata (Herrera et Mendoza, 1866)}

Cantharida ¿quadrinervata? Herrera et Mendoza, 1866: 265 (lapsus en el género) $(*)$

TERRA TYPICA: "Pachuca, Real del Monte, Mineral del Chico...". Según Selander (1983c) el tipo posiblemente está perdido.

Pyrota quadrinervata (Herrera et Mendoza, 1866): Dugès, 1886a: 582 (*)

COMENTARIOS TAXONÓMICOS: Especie incluida en el grupo de $P$. nobilis por Selander (1983c). Champion (1892) incluye en la sinonimia de $P$. quadrinervata dos nombres no disponibles: Pyrota ochracea Sturm, 1848: 174 y Pyrota quadripunctata Klug in Dejean 1837: 246. Dugès (1869a: 109) incluye el error tipográfico Cantharis cuadrinervata.

DisTRIBUCIÓN GEOGRÁFICA: Especie distribuida por el centro y norte de México (Vaurie, 1950; Selander, 1983c). Se ha citado de Chihuahua, Distrito Federal, Durango, Guanajuato, Hidalgo, México, Michoacán, Nuevo León, Puebla, Tamaulipas, Tlaxcala y Veracruz. Selander (1983c) considera erróneas las localidades de "Panamá" y "Atoyac, Mexico" que figuran en ejemplares de las colecciones del Zoologische Staatssammlung de Munich y del Carnegie Museum respectivamente.

MÉxICo: Chihuahua: Madera (Selander, 1983c); San José Babícora (Vaurie, 1950; Selander, 1983c). Distrito Federal: San Ángel (Selander, 1983c). Durango: Sierra de Durango (Selander, 1983c). Guanajuato: Tupátaro (Dugès, 1889e; Champion, 1892; Denier, 1934; Vaurie, 1950; Selander, 1983c). Hidalgo: Mineral del Chico (Herrera \& Mendoza, 1866; Dugès, 1869a; Champion, 1892; Denier, 1934; Vaurie, 1950; Selander, 1983c); Pachuca (Herrera \& Mendoza, 1866; Dugès, 1869a; Champion, 1892; Denier, 1934; Vaurie, 1950; Selander, 1983c); Real del Monte (Herrera \& Mendoza, 1866; Dugès, 1869a; Champion, 1892; Denier, 1934; Vaurie, 1950; Selander, 1983c). México: Chapingo (Selander, 1983c); San Miguel Acambay (Selander, 1983c); Toluca (Dugès, 1889e; Denier, 1934; Champion, 1892; Vaurie, 1950; Selander, 1983c). Michoacán: Pátzcuaro (Selander, 1983c). Nuevo León: Cola de Caballo (Selander, 1983c); Cerro Potosí, Galeana (Selander, 1983c). Puebla: Amalucan (Selander, 1983c); Chignahuapan (Selander, 1983c); Escuela Tecali (Selander, 1983c); Puebla (Selander, 1983c); Tlaltenango (Selander, 1983c). Tamaulipas: Ciudad Victoria (Selander, 1983c). Tlaxcala: Tlaxcala (Selander, 1983c). Veracruz: Coatepec (Champion, 1892; Denier, 1934; Vaurie, 1950; Selander, 1983c); Xalapa (Dugès, 1889e; Champion, 1892; Denier, 1934; Vaurie, 1950; Selander, 1983c).

\section{Pyrota rugulipennis Champion, 1892}

Pyrota rugulipennis Champion, 1892: 431 (*)

Terra TYPICA: "MeXICO, Canelas in Durango". Selander (1983c) designa lectotipo en la colección del British Museum of Natural History (Londres).

Cantharis rugulipennis (Champion, 1892): Champion, 1899: 186 (*)

COMENTARIOS TAXONÓmiCOS: Especie incluida en el grupo de $P$. nobilis por Selander (1983c). 
DISTRIBUCIÓN GEOGRÁFICA: Especie extendida desde el noroeste de México hasta El Salvador (Selander, 1983c). En México se ha citado en los estados de Chiapas, Durango, Guerrero, Jalisco, Morelos, Nayarit y Sinaloa. Según Selander (1983c) la cita de P. postica de Ahualulco (Jalisco) recogida por Dugès (1889e), Champion (1892) y Vaurie (1950) y transferida a $P$. plagiata por Werner (1954) corresponde en realidad a $P$. rugulipennis.

MÉxICo: Chiapas: Km 970 de la Carretera 190 (Selander, 1983c). Durango: Canelas (Champion, 1892; Denier, 1934; Vaurie, 1950; Selander, 1983c); Sierra de Durango (Selander, 1983c). Guerrero: Río Balsas (Selander, 1983c); Apaxtla (Selander, 1983c). Jalisco: Ahualulco (Dugès, 1889e sub $P$. postica; Champion, 1892 sub P. postica; Vaurie, 1950 sub $P$. postica Werner, 1954 sub P. plagiata; Selander, 1983c); Guadalajara (Selander, 1983c); Tuxpan (Selander, 1983c). Morelos: $12 \mathrm{mi}$. al este de Cuernavaca (Selander, 1983c). Nayarit: Jesús María (Selander, 1983c); La Mesa del Nayar (Selander, 1983c); 6 mi. al este de San Blas (Selander, 1983c); Tepic (Selander, 1983c). Sinaloa: $50 \mathrm{mi}$. al noreste de Mazatlán (Selander, 1983c); $42 \mathrm{mi}$. al este de Villa Unión (Selander, 1983c).

Pyrota tenuicostatis (Dugès, 1877)

Lytta vittigera LeConte, 1858a: 22 (non Pyrota vittigera Blanchard, 1843) (*)

TERRA TYPICA: "Near the boundary of Texas". La serie típica consta de dos ejemplares (fide LeConte, 1858a) en la colección de Museum of Comparative Zoology (Harvard University, Cambridge, EEUU) (fide Selander, 1983d).

Cantharis tenuicostatis Dugès, 1877: $60(*)$

TERRA TYPICA: “...del estado de Veracruz" originalmente; "Plan del Río, Veracruz, Mexico" tras designación de neotipo en la colección del British Museum of Natural History (Londres) (Selander, 1983d). Sin embargo Vázquez \& Zaragoza (1979) indican como tipo el ejemplar IBUNAM 010, de la colección del Instituto de Biología de la Universidad Nacional Autónoma de México.

Lytta tenuicosta (Dugès, 1877): Haag-Rutenberg, 1880: 50 (y error tipográfico) $(*)$

Pyrota dubitabilis Horn, 1885: 113 (nombre de substitución para L. vittigera LeConte, 1858) (*)

Pyrota tenuicostata (Dugès, 1877): Dugès, 1886a: 582 (enmienda injustificada) $(*)$

Pyrota tenuicostatis (Dugès, 1877): Dugès, 1889e: $91(*)$

Epicauta tenuicostalis (Dugès, 1877): Beauregard, 1890: 511 (y error tipográfico) $(*)$

Pyrota dubitalis Horn, 1885: Borchmann, 1917: 67 (lapsus) (*)

Comentarios taxonómicos: Dugès (1877) incluye el nombre no disponible Pyrota tenuicostatis Deyrolle en la sinonimia de C. tenuicostatis. Selander (1982b) incluye a esta especie en el grupo de $P$. tenuicostatis.

DISTRIBUCIÓN GEOGRÁFICA: Especie distribuida por el norte de México y sureste de los EEUU, citada también en Costa Rica (Selander, 1983d). Existe una cita del Río Grande, posiblemente en Texas (Horn, $1885 \mathrm{sub} P$. dubitabilis). En México se ha citado de los estados de Campeche, Chiapas, Coahuila, Nuevo León, Oaxaca, San Luis Potosí, Tabasco, Tamaulipas y Veracruz.

MÉXICo: Campeche: $25 \mathrm{mi}$. al E de Campeche (Selander, 1983d). Chiapas: Tuxtla Gutiérrez (Selander, 1983d); 6 mi. al
NO de Villa Flores (Selander, 1983d). Coahuila: 12 mi. al N de Hermanas (Selander, 1983d); $10 \mathrm{mi}$. al S de Sabinas (Selander, 1983d). Nuevo León: Apodaca (Selander, 1983d); Linares (Selander, 1983d); Monterrey (Selander, 1983d); Sabinas Hidalgo (Selander, 1983d). Oaxaca: sin precisar (Selander, 1983d). San Luis Potosí: Ciudad Valles (Selander, 1983d); El Naranjo (Selander, 1983d); El Salto (Selander, 1983d); Tamazunchale (Selander, 1983d). Tabasco: Teapa (Champion, 1892; Denier, 1934; Selander, 1983d); Tenosique (Selander, 1983d). Tamaulipas: Ciudad Mante (Selander, 1983d); Ciudad Victoria (Selander, 1983d); $47 \mathrm{~km}$ al S de Ciudad Victoria (Selander, 1983d); Güémez (Selander, 1983d); Santa Teresa (Selander, 1983d). Veracruz: Almolonga (Dugès, 1889e; Champion, 1892; Denier, 1934; Vázquez \& Zaragoza, 1979; Selander, 1983d); Atoyac (Selander, 1983d); Córdoba (Selander, 1983d); Fortín de las Flores (Selander, 1983d); Misantla (Selander, 1983d); Motzorongo (Selander, 1983d); Paso de Ovejas (Selander, 1983d); Plan del Río (Champion, 1892; Denier, 1934; Selander, 1983d); Playa San Vicente (Selander, 1983d); San Andrés Tuxtla (Champion, 1892; Denier, 1934; Selander, 1983d); La Tinaja (Tinajas) (Selander, 1983d); Veracruz (Dugès, 1889e).

Pyrota terrestris Selander, 1963

Pyrota terrestris Selander, 1963: 38 (*)

TERRA TYPICA: "18 mi. northwest of Yermo, 3800 feet ...". Holotipo en la colección del Field Museum of Natural History (Chicago, EEUU) (fide Selander, 1963, 1982b).

COMENTARIOS TAXONÓMICOS: Selander (1982b) la incluye en el grupo de $P$. mylabrina.

DISTRIBUCIÓN GEOGRÁFICA: Especie distribuida por el norte de México y extremo suroeste de los EEUU (Selander, 1964a; 1982b). En México se ha citado de Durango.

MÉXICo: Durango: Ceballos (Selander, 1982b); $18 \mathrm{mi}$. al NO de Yermo (Selander, 1963, 1964a, 1982b); 44 mi. al NO de Gómez Palacio (Selander, 1963, 1964a, 1982b); Tlahualillo de Zaragoza (Selander, 1963, 1982b).

\section{Pyrota trochanterica Horn, 1894}

Pyrota trochanterica trochanterica Horn, 1894

Pyrota trochanterica Horn, 1894: 439 (*)

TERRA TYPICA: "Sierra El Chinche 2,000 feet". Lectotipo, Horn no 164B, 10744, designado por Selander (1957b) en la colección de la California Academy of Sciences (San Francisco, EEUU).

Cantharis trochanterica (Horn, 1894): Champion, 1899: 190 (*)

Pyrota trochanterica werneri Selander, 1957

Pyrota trochanterica werneri Selander, 1957b: 140 (*)

TERRA TYPICA: "Guaymas, Sonora...". Holotipo en la colección de la California Academy of Sciences (San Francisco, EEUU) (fide Selander, 1957b)

COMENTARIOS TAXONÓMICOS: Especie que incluye dos subespecies $P$. $t$. trochanterica y $P$. trochanterica werneri.

Distribución geOgráfica: Pyrota $t$. trochanterica es exclusiva de México, mientras que $P$. $t$. werneri también se encuentra en el suroeste de los EEUU (Selander, 1957b; Werner et al., 1966). 
Pyrota trochanterica trochanterica: MÉXICO: Baja California Sur: $10 \mathrm{mi}$. al NO de La Paz (Selander, 1957b); Sierra El Chinche (Horn, 1894; Denier, 1934; Selander, 1957b).

Pyrota trochanterica werneri: MÉXICO: Sonora: Guaymas (Selander, 1957b; Werner et al., 1966); Empalme (Selander, 1957b); Hermosillo (Werner et al., 1966).

Subfamilia Nemognathinae Cockerell, 1910

Nemognathinae Cockerell, 1910: 307 (*)

CONTENIDO Y DISTRIBUCIÓN: La subfamilia está distribuida por la casi totalidad del Planeta. En el contienente americano, las 131 especies conocidas se agrupan en dos tribus: Horiini y Nemognathini (Pinto \& Bologna, 1999). En México existen representantes de estas dos tribus. Aunque el nombre Zonitinae Ganglbauer, 1907 se usó primero, dicho término ya se utilizaba para un grupo de moluscos (Cockerell, 1910).

Tribu Horiini Latreille, 1802

Horiales Latreille, 1802: $182(*)$

GÉNERO TIPO: Horia Fabricius, 1787, único género incluido por Latreille (1802) en la definición de la tribu.

CONTENIDO Y DISTRIBUCIÓN: En el continente americano únicamente se conocen dos especies agrupadas en el género Cissites (Pinto \& Bologna, 1999).

\section{Género Cissites Latreille, 1804}

Cissites Latreille, 1804a: 154

ESPECIE TIPO: Horia maculata (= Cucujus maculatus Swederus, 1787) por monotipia. La publicación de Latreille (1804a: 154, 199) tiene al parecer prioridad sobre Latreille (1804b: 364) aunque ésta sea la referencia utilizada generalmente (Selander, 1991)

CONTENIDO Y DISTRIBUCIÓN: El género está representado por dos especies, que se extienden desde Colombia hasta el norte de la Argentina y las Islas Galápagos en América del Sur, y desde Panamá hasta el sur de los Estados Unidos, y las Antillas, en América del Norte (Selander \& Bouseman, 1962; Pinto \& Bologna, 1999). Ha sido introducido, aparentemente sin éxito, en las Islas Hawaii (Bianchi, 1962; Pinto \& Bologna, 1999).

Cissites auriculata (Champion, 1892)

Horia auriculata Champion, 1892: $372(*)$

TERrA TYPICA: "North AMERICA, South-western Arizona.- MeXICO, Mazatlan, San Blas..., Acapulco..., Guadalajara, Almolonga..., Tasco, Oaxaca...; Guatemala, Cerro Zunil, San Isidro, San Gerónimo...; Costa Rica...- Antilles, Barbados". Tipo no designado; la serie típica se encuentra posiblemente en la colección del British Musesum of Natural History.

Cissites auriculata (Champion, 1892): Gahan, 1908: 203 (*)

COMENTARIOS TAXONÓMICOS: Ocasionalmente confundida con C. maculata.

DisTRIBUCIÓN GEOGRÁFICA: Especie distribuida desde México y las Antillas, hasta Costa Rica, con citas en Guatemala y Nicaragua (Enns, 1958; Maes, 1989; Maes $\&$ Huether, 2007). Existe una cita confirmada en Texas y otra dudosa en Arizona (Enns, 1958; Lewis, 2004), mientras que Enns (1958) y Selander \& Bouseman (1960) consideran que las citas de esta especie en las Antillas (Champion, 1892; Leng \& Mutchler, 1914) son cuestionables. En México se ha citado de Colima, Guerrero, Jalisco, Morelos, Nayarit, Oaxaca, Puebla, Sinaloa y Veracruz. Champion (1892) considera que las citas de C. maculata de Colima (Dugès, 1869a, 1889e) corresponden a $C$. auriculata.

MÉXICO: Colima: Colima (Dugès, 1869a, 1889e sub $H$. maculata; Champion, 1892; Betrem, 1932; Enns, 1958). Guerrero: Acapulco (Champion, 1892; Enns, 1958); Balsas (Betrem, 1932; Enns, 1958); Taxco (Champion, 1892; Enns, 1958). Jalisco: Guadalajara (Champion, 1892; Enns, 1958). Morelos: Cuernavaca (Dugès, 1869a; Cros, 1928; MacSwain, 1956; Enns, 1958). Nayarit: San Blas (Champion, 1892; Enns, 1958). Oaxaca: Oaxaca (Champion, 1892; Enns, 1958). Puebla: Tehuacán (Betrem, 1932; Enns, 1958). Sinaloa: Mazatlán (Champion, 1892; Enns, 1958). Veracruz: Almolonga (Champion, 1892; Enns, 1958).

Cissites maculata (Swederus, 1787)

Cucujus maculatus Swederus, 1787: 199

TERRA TYPICA: Cayenne (fide Beauregard, 1890); New York Americae (fide Selander \& Bouseman, 1960). Tipos en paradero desconocido (fide Salander \& Bouseman, 1960).

Horia maculata (Swederus, 1787): Fabricius, 1792: $90\left(^{*}\right)$

Cissites maculata (Swederus, 1787): Latreille, 1804: 364 (*)

Cantharis maculata (Swederus, 1787): Voet, 1806: 76

Horia apicalis Perty, 1830: 66 (*)

TERRA TYPICA: “...in Serra do Caraca, Prov. Minarum.”.

Cissites apicalis (Perty, 1830): Gahan, 1908: 203 (*)

Cissites maculata var. championi Pic, 1929: 81 (*)

TERRA TYPICA: "Colombia". La descripción se basa en la que presenta Champion (1892: 37).

Cissites maculata var. intermedia Betrem, 1932: 87 (*)

TERRA TYPICA: "Columbien". Holotipo en la colección del Nationaal Natuurhistorisch Museum de Leiden (Naturalis) (fide Betrem, 1932)

Comentarios taxonómicos: Hasta el trabajo de Champion (1892) en esta especie se venía incluyendo $C$. auriculata.

DisTRIBUCIÓN GEOGRÁFICA: Especie que se considera distribuida desde México y las Antillas hasta Argentina (Latreille, 1804; Selander \& Bouseman, 1960; Genaro, 1996), aunque la única cita comprobada existente en México (Michoacán: Morelia, Champion, 1892) podría corresponder a un error de etiquetado o a una introducción accidental. Enns (1958) señaló por error su presencia en Morelos y Champion (1892) indica que las citas de Colima de Dugès $(1869 \mathrm{a} ; 1889 \mathrm{e})$ corresponden a $C$. auriculata. Werner et al. (1966) señalan que las citas de Arizona (EEUU) son muy dudosas y que requieren confirmación. Por todos estos datos, cabe la posibilidad de eliminar a $C$. maculata del catálogo de especies presentes en México. 1892).

MÉXICO: Michoacán: Morelia (posible error) (Champion, 
Tribu Nemognathini Lacordaire, 1859

Némognathides Lacordaire, 1859: $690(*)$

GÉNERO TIPO: Nemognatha Illiger, 1807.

CONTENIDO Y DISTRIBUCIÓN: La tribu está distribuida por la casi totalidad del Planeta. En el contienente americano, las 129 especies conocidas se agrupan en 8 géneros, que a excepción de Tricrania LeConte, 1860, que sólo se conoce de los Estados Unidos y Zonitolytta Pic, 1927, exclusivo de América del Sur, incluyen representantes en México (Pinto \& Bologna, 1999). Los géneros representados en México son Gnathium, Hornia, Nemognatha, Pseudozonitis, Rhyphonemognatha y Zonitis. Dugès (1889b) propuso la tribu Hornii (en realidad con carácter de subfamilia ) para incluir a los géneros Hornia y Leonia, y Blackwelder (1945) utilizó la tribu Horniini para incluir a los mismos taxones.

Como indican Pinto \& Bologna (1999) y Pinto (2001), hasta el momento no se ha realizado una revisión de conjunto adecuada de los Nemognathini americanos por lo que la definición de los géneros y la asignación de especies, sobre todo las neotropicales, a un género u otro es problemática. En lo tocante a la fauna mexicana, estos problemas son particularmente serios en el conjunto Nemognatha - Pseudozonitis - Zonitis de las regiones meridionales, ya que la última clasificación genérica es la de Enns (1956), para cuya realización este autor incluyó casi exclusivamente representantes de la fauna de los Estados Unidos. En este catálogo, la asignación genérica de muchas de las especies se basa exclusivamente en las descripciones originales por lo que, hasta que no se realice un examen de los ejemplares tipo, estas adscripciones han de considerarse provisionales.

Género Gnathium Kirby, 1818

Gnathium Kirby, 1818: 425

ESPECIE TIPO: Gnathium francilloni Kirby, 1818, por monotipia (fide MacSwain, 1952; Selander, 1991; Pinto \& Bologna, 1999).

COMENTARIOS TAXONÓMICOS: Say (1824b: 307) utiliza erróneamente el nombre Gnatho, mientras que Dugès (1889e: 113, 194) desliza los errores tipográficos Grathium y Gnahtium a la vez que se equivoca en la adscripción del género.

CONTENIDO Y DISTRIBUCIÓN: El género está representado por 14 especies, que se extienden por el norte de México y el sur de los Estados Unidos (Pinto \& Bologna, 1999). Existe una cita en Nicaragua (Maes, 1989) que no corresponde a este género (Pinto \& Bologna, 1999; Maes $\&$ Huether, 2007). En México únicamente se han citado tres especies de este género, pero podrían encontrarse algunas más que se han citado en California, Nuevo México y Texas (MacSwain, 1952), muy cerca de la frontera.

Gnathium francilloni Kirby, 1818

Gnathium francilloni Kirby, 1818: 426

TERRA TYPICA: Georgia (fide Vaurie, 1950)
Nemognatha flavicollis LeConte, 1858a: 23 (*)

TERRA TYPICA: “...Texas..."

Gnathium flavicolle (LeConte, 1858): Horn, 1870: 95 (non Chevrolat, 1829-44) (*)

COMENTARIOS TAXONÓMICOS: Beauregard (1890) aún trataba a G. francilloni y G. flavicolle como especies distintas y Horn (1870) presenta una tabla comparativa para la diferenciación de ambos taxa. MacSwain (1952) indica que, debido a la presencia de poblaciones con caracteres intermedios, es posible que G. vandykei pueda ser una subespecie de $G$. francilloni.

DISTRIBUCIÓN GEOGRÁFICA: Especie extendida por el norte de México y una amplia región de los EEUU (Selander, 1954b; Werner et al., 1966). En México se ha citado en los estados de Durango y Zacatecas.

MÉXICO: Durango: Ciudad Durango (Champion, 1892; Vaurie, 1950). Zacatecas: 19 mi. al S de Sombrerete (Selander, 1954b).

Gnathium minimum (Say, 1824)

Nemognatha minima Say, 1824b: 306 (*)

TERRA TYPICA: "Arkansa”, “...near the Rocky Mountains”. Tipos posiblemente perdidos.

Gnathium walckenaeri Laporte de Castelnau, 1840: 281

TERRA TYPICA: Veracruz (fide Champion, 1892)

Nemognatha longicollis LeConte, 1858b: 77 (*)

TERRA TYPICA: "Ringgold Barracks, Texas". Tipos posiblemente en el Museum of Comparative Zoology (Harvard University, Cambridge, EEUU).

Gnathium minimum (Say, 1824b): Horn, 1870: 94 (*)

Gnathium longicolle (LeConte, 1858): Beauregard, 1890: 469 (*)

COMENTARIOS TAXONÓMICOS: Beauregard (1890) trató a $G$. minimum, $G$. walckenaeri y $G$. longicolle como especies distintas. MacSwain (1952) indica que, aunque Champion (1892) incluye en la sinonimia de G. minimum a $G$. walckenaeri y a $G$. longicolle, su estatus ha de ser revisado. En caso de no ser sinónimos, G. minimum posiblemente no se encontraría en México. Beauregard (1890: 469) escribe erróneamente Gnathium walkenaeri.

DISTRIBUCIÓN GEOGRÁFICA: Especie distribuida por el norte de México y una amplia región de los EEUU y Canadá (MacSwain, 1952; Werner et al., 1966). En México se ha citado de Coahuila, Sonora y Veracruz.

MÉxICo: Coahuila: San Pedro de Colonias (Vaurie, 1950). Sonora: Norte de Sonora (Champion, 1892; Vaurie, 1950). Veracruz: Veracruz (Laporte de Castelnau, 1840 sub G. walckenaeri; Champion, 1892; Vaurie, 1950).

\section{Gnathium nitidum Horn, 1870}

Gnathium nitidum Horn, 1870: 95 (*)

TERRA TYPICA: "Owen's Valley, California". Tipos posiblemente en la colección del Museum of Comparative Zoology de Harvard (EEUU).

COMENTARIOS TAXONÓMICOS: Los ejemplares del centro de California (EEUU) parecen diferir a nivel subespecífico (MacSwain, 1952).

DisTRIBUCIÓN GEOGRÁFICA: Especie distribuida por el norte de México y el sur de los EEUU (MacSwain, 1952; 
Werner et al., 1966). En México se ha citado de Baja California, Coahuila y Durango.

MÉxICO: Baja California: sin precisar (MacSwain, 1952). Coahuila: Guadalupe (Vaurie, 1950); La Gloria, al sur de Monclova (Vaurie, 1950). Durango: Villa Lerdo (Champion, 1892; Vaurie, 1950).

Género Hornia Riley, 1877

Hornia Riley, 1877b: 564 (*)

ESPECIE TIPO: Hornia minutipennis Riley, 1877, por monotipia.

Leonia Dugès, 1889f: 211 (non Gray, 1840, nec Baird, 1850) (*)

ESPECIE TIPO: Leonia rileyi Dugès, 1889, por monotipia.

Leonidia Cockerell, 1900: 11 (nombre substituto de Leonia Dugès, 1889) (*)

COMENTARIOS TAXONÓMICOS: Mickel (1928: 38) escribe por error Leonidea.

CONTENIDO Y DISTRIBUCIÓN: El género está representado por 3 especies, que se extienden por México y el sur de los Estados Unidos (Van Dyke, 1928; Linsley, 1942; Pinto \& Bologna, 1999). Existe una cita en El Ecuador (Denier, 1935a) que requiere confirmación (Pinto \& Bologna, 1999). De las tres especies conocidas, $H$. minutipennis Riley, 1877, H. boharti Linsley, 1942 y H. mexicana (Dugès, 1889), únicamente esta última parece encontrarse en México, aunque las otras dos se han localizado en California (EEUU) por lo que también podrían encontrarse en territorio mexicano.

\section{Hornia mexicana Dugès, 1886}

Hornia mexicana mexicana Dugès, 1886

Hornia mexicana Dugès, 1886a: 579 (*)

TERRA TYPICA: No indicada.

Leonia rileyi Dugès, 1889f: $211\left({ }^{*}\right)$

TERRA TYPICA: "Hacienda de Jupátaro [sic], State of Guanajuato, Mexico"; en una publicación posterior Dugès (1889a) corrige el error e indica "Hacienda de Tupátaro, Estado de Guanajuato". Un sintipo en paradero desconocido, el otro en la colección Sallé (posiblemente en el British Museum of Natural History)(fide Dugès, 1889a).

Leonidia mexicana (Dugès, 1886): Borchmann, 1917: 138 (*)

Leonidia rileyi (Dugès, 1889): Borchmann, 1917: 138 (*)

Hornia rileyi (Dugès, 1889): Mickel, 1929: 7 (*)

Hornia mexicana blomi MacSwain, 1958

Hornia mexicana blomi MacSwain, 1958: 395 (*)

TERRA TYPICA: "San Cristobal las Casas, Chiapas, México". Holotipo en la colección de la California Academy of Sciences (San Francisco, EEUU); la serie típica consta de 218 ejemplares más (fide MacSwain, 1958).

COMENTARIOS TAXONÓMICOS: Especie constituida por tres subespecies H. m. mexicana, H. m. blomi y H. m. neomexicana (Cockerell, 1899). Van Dyke (1928) estableció la sinonimia de $H$. gigantea con $H$. minutipennis. MacSwain (1958) trata a la hasta entonces especie independiente $H$. neomexicana (ver Mickel, 1928; Linsley, 1942) como subspecie de $H$. mexicana.

DisTRIBUCIÓN GEOGRÁFICA: Especie posiblemente distribuida por todo México y el sur de los EEUU (MacSwain, 1958). En México la especie se ha citado de
Chiapas y Durango. La subespecie H. m. neomexicana no se ha encontrado en México, aunque seguramente está presente en el norte.

Hornia mexicana mexicana: MÉxICo: Guanajuato: Hacienda Tupátaro (Dugès, 1889f; Champion, 1892 sub $L$. rileyi; MacSwain, 1958).

Hornia mexicana blomi: MÉXICo: Chiapas: San Cristóbal de las Casas (MacSwain, 1958).

Género Nemognatha Illiger, 1807

Nemognatha Illiger, 1807: $333(*)$

ESPECIE TIPO: Zonitis vittata Fabricius, 1801 (= Zonitis piazata Fabricius, 1798) por monotipia.

Nematognatha Gemminger et Harold, 1870: 2163 (enmienda injustificada) (*)

Gnathonemula Aksentjiev, 1981: 862 (*)

ESPECIE TIPO: Gnathonemula gracilis Aksentjev, 1981 por monotipia.

CONTENIDO Y DISTRIBUCióN: El género, que incluye 77 especies, está extendido por todo el Planeta excepto en Oceanía. Las 49 especies americanas se distribuyen por todo el continente excepto en las zonas de mayor altitud (Pinto \& Bologna, 1999). El género Zonitolytta Pic, 1927, fue tratado por Selander (1991) como subgénero de Nemognatha, pero Pinto \& Bologna (1999) lo mantienen como género independiente. En la bibliografía antigua, muchas especies incluidas actualmente en Nemognatha se incluían en Zonitis y viceversa.

Las especies norteamericanas se reparten en 4 subgéneros: Nemognatha, Meganemognatha, Pauronemognatha y Pronemognatha, pero varias de las especies mexicanas, particularmente las neotropicales no han sido asignadas a un subgénero concreto. Estas especies sin adscripción subgenérica revisada, que incluso podrían pertenecer a otros géneros, se indican a continuación.

Nemognatha (?) chrysomeloides (Linnaeus, 1763)

Meloe chrysomeloides Linnaeus, 1763: 396 (página en la reimpresión de 1789)

TERRA TYPICA: "Surinami" (fide Linnaeus, 1787, 1789).

Cantharis chrysomeloides (Linnaeus, 1763): Thunberg, 1784: 17

Lytta chrysomeloides (Linnaeus, 1763): Schoenherr, 1817: 28

Nemognathus coeruleipennis Perty, 1830: 67 (*)

TERRA TYPICA: “...ad flumen S. Francisci."

Nemognatha versicolor Chevrolat, 1834b: fasc. $4, \mathrm{n}^{\circ} 82$ (sin paginar) (*) TERRA TYPICA: "environs de Mexico".

Nemognatha abdominalis Lucas in Laporte de Castelnau, 1859: 148 (*) TERRA TYPICA: "environs de Rio de Janeiro".

Nemognatha bicolor Lucas in Laporte de Castelnau, 1859: 148 (non LeConte, 1853; nec Walker, 1866) (*)

TERRA TYPICA: "environs de Rio de Janeiro".

Nematognatha lucasi Gemminger, 1870: 124 (nombre de substitución para Nemognatha bicolor Lucas in Laporte de Castelnau, 1859 y error en el género) $(*)$

Nemognatha atra Beauregard, 1890: 465 (non Zonitis atrum Schwartz, 1808 , nec Gnathium atrum Dugès, 1889) (*) (syn. nov.)

TERRA TYPICA: "Brésil". El tipo se encontraría en la colección del Museo de Bruselas bajo el nombre de $N$. atra Buquet (in litt.) (fide Beauregard, 1890).

Nemognatha coeruleipennis var. fulviventris Beauregard, 1890: 466 (*) TERRA TYPICA: "Cayenne". El tipo se encontraría en la colección del Museo de Bruselas bajo el nombre de N. fulviventris Deyr. (in litt.) (fide Beauregard, 1890). 
Nemognatha pallidicollis Beauregard, 1890: 466 (*) (syn. nov.)

TERRA TYPICA: "Brésil". El tipo se encontraría en la colección del Museo de Bruselas bajo el nombre de N. pallidicollis Buquet (in litt.) (fide Beauregard, 1890).

Nemognatha violacea Beauregard, 1890: 468 (*) (syn. nov.)

TERra TYPICA: "Brésil". El tipo se encontraría en la colección del

Museo de Bruselas bajo el nombre de $N$. violacea Deyr. (in litt.) (fide Beauregard, 1890)

Nemognatha beauregardi Pic, 1910: 7 (nombre de sustitución para $N$. atra Beauregard, 1890) (*)

Zonitis chrysomeloides (Linnaeus, 1763): Denier, 1935a: 148 (*)

Zonitis beauregardi (Pic, 1910): Blackwelder, 1945: 481 (*)

Nemognatha chrysomeloides ab. markli Kaszab, 1963a: 344 (*)

TERRA TYPICA: "Peru: Sullana, Hda. Mallares...". El tipo se encontraría en la colección del Naturhistorischen Museum de Basel (fide Kaszab, 1963a).

COMENTARIOS TAXONÓMICOS: Especie con una historia taxonómica muy complicada, con cambios de género diversos (Gemminger \& Harold, 1870; Borchmann, 1917), hasta el punto de que Beauregard, (1890: 514) dudando entre incluir el nombre $N$. chrysomeloides en el género Epicauta o en Cantharis, finalmente lo incluye como "incertae sedis". El problema se complica aún más con la existencia del nombre Zonitis abdominalis Laporte de Castelnau, 1840, también de sinonimia compleja, distinto de Nemognatha abdominalis Lucas in Laporte de Castelnau, 1859, que es un sinónimo de la especie que nos ocupa. Beauregard (1890) incluyó como sinónimo de $N$. coeruleipennis a Nemognatha cyanipennis Dejean, 1837: 249, que posiblemente sea un nombre no disponible. Martínez (1992) considera especies distintas a $N$. coeruleipennis y a $N$. chrysomeloides y utiliza el primero de estos nombres para referirse al material argentino.

Las diferencias que señala Beauregard (1890) entre $N$. pallidicollis, $N$. atra y $N$. coerulipennis se refieren sobre todo a la longitud de las maxilas, a la forma del pronoto, a la coloración y al tamaño, carácteres muy variables en el grupo, que no parecen suficientes para mantener la validez específica de los taxones involucrados, sobre todo porque Beauregard (1890: 514) no conocía $N$. chrysomeloides, por lo que aquí incluimos estos nombres, provisionalmente y en espera de la revisión de las series típicas, en la sinonimia de $N$. chrysomeloides.

DisTRIBUCIÓN GEOGRÁFICA: Champion (1892), seguido por Blackwelder (1945), indica que la especie se distribuye por México, Guatemala, Surinam, Guayana Francesa, Argentina, Brasil y Perú. Martínez (1992) añade nuevos datos para La Argentina. En México se ha citado en el Distrito Federal, Chiapas, Guerrero, Jalisco, Michoacán, Puebla, Sinaloa y Veracruz.

MÉxICo: Chiapas: cercanías de la Estación Biológica Chajul, 1607'N, 9057'O, Selva Lacandona (Yanega \& Leschen, 1994); cruce del Río La Venta con la carretera Panamericana, entre Cintalapa y Ocozocuautla (Linsley et al., 1980); Francia, $12.5 \mathrm{~km}$ al NE de Cintalapa (Linsley et al., 1980); Soyalo (Linsley et al., 1980); Tuxtla Gutiérrez (Linsley et al., 1980).
Distrito Federal: Ciudad de México (Chevrolat, 1834b; Dugès, 1870; Champion, 1892). Guerrero: Tierra Colorada (Champion, 1892); Venta del Zopilote (Champion, 1892). Jalisco: Rancho de la Garita, Sierra de Mazamitla (Dugès, 1870; Champion, 1892). Michoacán: Sur del Estado (Dugès, 1889e). Puebla: Izúcar de Matamoros (Champion, 1892). Sinaloa: $18 \mathrm{~km}$ al N de Culiacán (Parker, 1977; Linsley et al., 1980). Veracruz: Veracruz (Dugès, 1889 e sub $N$. versicolor).

Nemognatha (?) ephippiata Champion, 1892

Nemognatha ephippiata Champion, 1892: 377 (*)

TeRRa tyPICA: "MeXICO, Rincon in Guerrero, Teapa in Tabasco...; Guatemala, Chiacam in Vera Paz..., between Tactic and Tucuru...;

Panama, Bugaba...”. Ocho sintipos, posiblemente en la colección del British Museum of Natural History.

Nematognatha ephippiata (Champion, 1892): Champion, 1899: 205 (*) Zonitis ephippiata (Champion, 1892): Denier, 1935a: 148 (*)

COMENTARIOS TAXONÓMICOS: Especie con gran variabilidad en la longitud de las maxilas, por lo que Champion (1892) indica que consituye un paso transicional entre los géneros Nemognatha y Zonitis.

DistrIBUCIÓN GEOGRÁfICA: Especie conocida en México, Guatemala y Panamá (Champion, 1892). En México se ha citado en Guerrero y Tabasco.

MÉxICO: Guerrero: Rincón (Champion 1892). Tabasco: Teapa (Champion 1892).

Nemognatha (?) foveifrons Champion, 1892

Nemognatha foveifrons Champion, 1892: 376 (*)

TERRA TYPICA: "MeXICO, Atoyac in Vera Cruz....". Holotipo posiblemente en la colección del British Museum of Natural History.

Nematognatha foveifrons (Champion, 1892): Champion, 1899: 205 (*)

Zonitis foveifrons (Champion, 1892): Denier, 1935a: $148\left({ }^{*}\right)$

DistRIBUCIÓN GEOGRÁFICA: Especie endémica de México sólo conocida de Veracruz.

MÉxICo: Veracruz: Atoyac (Champion, 1892).

Subgénero Nemognatha Illiger, 1807

Nemognatha Illiger, 1807: 333 (*)

ESPECIE TIPO: Zonitis vittata Fabricius, 1801 (= Zonitis piazata Fabricius, 1798) por monotipia.

Nematognatha Gemminger et Harold, 1870: 2163 (enmienda injustificada) $(*)$

Gnathonemula Aksentjiev, 1981: 862 (*)

ESPECIE TIPO: Gnathonemula gracilis Aksentjev, 1981 por monotipia.

CONTENIDO Y DISTRIBUCIÓN: El subgénero incluye dos especies en América del Norte (Enns, 1956), de las cuales $N$. (N.). piazata está presente en México. Es muy posible que, además, se encuentre también $N$. (N.) bifoveata Enns, 1956, ya que se ha citado muy cerca de la frontera con los EEUU (Werner et al., 1966).

Nemognatha (Nemognatha) piazata (Fabricius, 1798)

Zonitis piazata Fabricius, 1798: 104 (*)

TERRA TYPICA: "Carolina". Holotipo posiblemente en la colección del Muséum National d'Histoire Naturelle (Paris) (fide Enns, 1956). Nemognatha piezata (Fabricius, 1798): LeConte, 1853: 347 (y error) (*)

Nemognatha (Nemognatha) piazata bicolor LeConte, 1853 Nemognatha bicolor LeConte, 1853: 345 (*) 
TERRA TYPICA: "Missouri Teritory". Holotipo $n^{\circ}$ 4951, en la colección del Museum of Comparative Zoology (Harvard University, Cambridge, EEUU) (fide Enns, 1956).

Nemognatha texana LeConte, 1853: 347 (*)

TERRA TYPICA: “Texas". Holotipo $n^{\circ} 4962$, en la colección de Museum of Comparative Zoology (Harvard University, Cambridge, EEUU) (fide Enns, 1956)

Nemognatha discolor LeConte, 1858b: 77 (*)

TERRA TYPICA: "Texas". Holotipo $\mathrm{n}^{\circ} 4958$, en la colección del Museum of Comparative Zoology (Harvard University, Cambridge, EEUU) (fide Enns, 1956).

Nemognatha bicolor var. stellaris Beauregard, 1890: 465 (*)

TERRA TYPICA: "Californie"

Zonitis piezata var. bicolor (LeConte, 1853): Denier, 1935a: $149(*)$

COMENTARIOS TAXONÓMICOS: El nombre $N$. piezata también se ha usado en muchas obras anteriores a LeConte (1853) para $N$. piazata. Beauregard (1890) ya incluye a $N$. texana en la sinonimia de $N$. piazata.

DISTRIBUCIÓN GEOGRÁFICA: Especie con tres subespecies: la nominal, localizada en el sureste de los EEUU, N. l. palliata LeConte, 1853, distribuida por el norte de los EEUU, y $N$. $l$. bicolor que se extiende por el norte de México y por una amplia región del centro de los EEUU (Enns, 1956). Existe una cita a confirmar de Horn (1894 sub N. piezata) en San Luis (Baja California).

MÉxICo: Chihuahua: Delicias (Vaurie, 1950 sub Z. piezata). Veracruz: Xalapa (Champion, 1892 sub N. piezata; Vaurie, 1950 sub Z. piezata); Atoyac (Champion, $1892 \mathrm{sub}$ N. piezata; Vaurie, $1950 \mathrm{sub} Z$. piezata).

\section{Subgénero Meganemognatha Enns, 1956}

Meganemognatha Enns, 1956: 723 (*)

ESPECIE TIPO: Nemognatha lurida LeConte, 1853, por designación original.

CONTENIDO Y DISTRIBUCIÓN: El subgénero incluye 15 especies en América del Norte (Enns, 1956).

En México se ha citado la presencia de cinco especies: $N$. (M.). bridwelli, $N$. (M.). explanata, $N$. (M.). lurida, $N$. (M.). lutea y N. (M.). pallens. Además de estas especies, podrían encontrarse también, ya que se han citado en EEUU muy cerca de la frontera, las siguientes: N. (M.) hurdi MacSwain, 1951; N. (M.) macswaini Enns, 1956; N. (M.) meropa Enns, 1956; N. (M.) miranda Enns, 1956 y N. (M.) nitidula Enns, 1956.

Nemognatha (Meganemognatha) bridwelli Wellman, 1912 Nemognatha bridwelli Wellman, 1912: 37 (*)

TERRA TYPICA: "Meloland, Imperial Valley, California". Tres sintipos en paradero desconocido (fide Enns, 1956).

Zonitis bridwelli (Wellman, 1912): Denier, 1935a: 147 (*)

DISTRIBUCIÓN GEOGRÁFICA: Especie distribuida por el extremo noroeste de México y el suroeste de los EEUU (Enns, 1956; Werner et al., 1966). En México se ha citado únicamente en Baja California.

MÉXICo: Baja California: Tecolote (Werner et al., 1966).

Nemognatha (Meganemognatha) explanata Enns, 1956 Nemognatha (Meganemognatha) explanata Enns, 1956: 745 (*)
TERRA TYPICA: “...Bishop, Texas...”. Holotipo $n^{\circ}$ 62732, en la colección del Snow Entomological Museum (University of Kansas, EEUU) (fide Enns, 1956). La serie típica consta de numerosos ejemplares más (Enns, 1956).

DISTRIBUCIÓN GEOGRÁFICA: Especie distribuida por el norte de México y Texas (EEUU) (Enns, 1956). En México se ha citado de Coahuila.

MÉxICo: Coahuila: Saltillo (Enns, 1956).

Nemognatha (Meganemognatha) lurida LeConte, 1853 Nemognatha (Meganemognatha) lurida lurida LeConte, 1853 Nemognatha lurida LeConte, 1853: 345 (*)

TERRA TYPICA: "Missouri Teritory, on Platte River". Lectotipo $\mathrm{n}^{\circ}$ 4952, designado por Enns (1956) en la colección del Museum of Comparative Zoology (Harvard University, Cambridge, EEUU) (fide Enns, 1956).

Nemognatha rufa Dugès, 1889: 111 (*)

TERRA TYPICA: "Villa Lerdo". Tipo perdido según Enns (1956), pero Vázquez \& Zaragoza (1979) indican como tipo el ejemplar n 2888 (IBUNAM 031) de la colección del Instituto de Biología de la Universidad Nacional Autónoma de México.

Nematognatha rufa (Dugès, 1889): Champion, 1899: 205 (*)

Zonitis lurida (LeConte, 1853): Denier, 1935a: 148 (*)

Nemognatha (Meganemognatha) lurida apicalis LeConte, 1853 Nemognatha apicalis LeConte, 1853: 345 (*)

TERRA TYPICA: “...Benicia, California, and ...Texas...”; "Benicia, California" tras designación del ejemplar no 4954 de la colección del Museum of Comparative Zoology (Harvard University, Cambridge, EEUU) como lectotipo (Enns, 1956).

Nemognatha bicolor Walker, 1866: 331 (non LeConte, 1853; nec Lucas, 1859)

TERRA TYPICA: "British Columbia". Tipo en el British Museum of Natural History (fide LeConte, 1870).

Nemognatha walkeri Beauregard, 1889: 212 (nombre de substitución para Nemognatha bicolor Walker, 1866) (*)

Zonitis apicalis (LeConte, 1853): Denier, 1935a: 147 (*)

COMENTARIOS TAXONÓMICOS: Especie con dos subespecies, la nominal y N. l. apicalis (Enns, 1956). Borchmann (1917) trata a $N$. apicalis como especie independiente. Beauregard (1890) trata a $N$. decipiens LeConte, 1853, como variedad de $N$. lurida, mientras que Champion (1892), Borchmann (1917), Denier (1935a) y Vaurie (1950) incluyeron a $N$. decipiens y $N$. rufa en la sinonimia de $N$. lurida, pero $N$. decipiens es considerada una especie independiente por Enns (1956). LeConte (1870) establece la sinonimia de $N$. bicolor Walker con $N$. l. apicalis.

DisTRIBUCIÓN GEOGRÁFICA: Nemognatha l. apicalis ha sido citada en el norte de la Península de Baja California (Horn, 1894). La subespecie N. l. lurida se extiende por el norte de México y por una amplia región del centro de los EEUU (Enns, 1956).

Nemognatha (Meganemognatha) lurida lurida: MÉXICO: Chihuahua: 2 mi. al NE de Camargo (Selander, 1954b); Delicias (Vaurie, 1950). Coahuila: Monclova (Champion 1892; Vaurie, 1950); San Pedro de Colonias (Vaurie, 1950); Guadalupe (Vaurie, 1950). Durango: Villa Lerdo (Dugès, 1889e sub N. rufa; Champion 1892; Vaurie, 1950; Vázquez \& Zaragoza, 1979). Sonora: Norte de Sonora (Champion 1892; Vaurie, 1950). 
Nemognatha (Meganemognatha) lurida apicalis: MÉXICO: Baja California: San Luis (Horn, 1894).

Nemognatha (Meganemognatha) lutea LeConte, 1853 Nemognatha (Meganemognatha) lutea lutea LeConte, 1853 Nemognatha lutea LeConte, 1853: 346 (*)

TERRA TYPICA: "Missouri Teritory". Lectotipo ${ }^{\circ}$ 4952, designado por Enns (1956) en la colección del Museum of Comparative Zoology (Harvard University, Cambridge, EEUU) (fide Enns, 1956).

Zonitis lutea (LeConte, 1853): Denier, 1935a: 149 (*)

DISTRIBUCIÓN GEOGRÁFICA: Especie con tres subespecies, la nominal presente en México, $N$. $l$. dichroa LeConte, 1853, distribuida por el suroeste del Canadá y noroeste de los EEUU y $N$. l. dubia LeConte, 1853, que aunque no ha sido citada en México podría encontrarse en el norte de Baja California. La subespecie $N$. l. lutea se extiende por el norte de México y por una amplia región del centro de los EEUU (Enns, 1956).

MÉxICo: Chihuahua: $92 \mathrm{~km}$ al N de Chihuahua (Vaurie, 1950); $239 \mathrm{~km}$ al S de Ciudad Juárez (Vaurie, 1950). Sonora: Norte de Sonora (Champion 1892; Vaurie, 1950).

Nemognatha (Meganemognatha) pallens LeConte, 1853 Nemognatha pallens LeConte, 1853: 346 (*)

TERRA TYPICA: "Vallecitas, in the southern part of California". Lectotipo $n^{\circ} 4956$, designado por Enns (1956) en la colección del Museum of Comparative Zoology (Harvard University, Cambridge, EEUU) (fide Enns, 1956).

COMENTARIOS TAXONÓMICOS: Incluida en la sinonimia de $N$. lutea (Beauregard, 1890), considerada como especie independiente por Enns (1956).

DisTRIBUCIÓN GEOGRÁFICA: Especie distribuida en Alta California (EEUU) que podría encontrarse en el norte de Baja California y a la que, según Enns (1956), podrían corresponder algunas citas de Champion (1892) efectuadas bajo el nombre de $N$. lutea.

Subgénero Pauronemognatha Enns, 1956

Pauronemognatha Enns, 1956: 764 (*)

ESPECIE TIPO: Nemognatha nigripennis LeConte, 1853, por designación original.

CONTENIDO Y DISTRIBUCIÓN: El subgénero incluye 7 especies en América del Norte (Enns, 1956).

En México se encuentran al menos cinco especies de este subgénero, $N$. (P.). brevicollis, $N$. (P.). nigripennis, $N$. (P.). scutellaris, $N$. (P). scutellaroides y $N$. (P). selloa.

\section{Nemognatha (Pauronemognatha) brevicollis Champion,} 1892

Nemognatha brevicollis Champion, 1892: 376 (*)

TERRA TYPICA: "MeXICO, Matamoros Izúcar". Dos sintipos posiblemente en la colección del British Museum of Natural History (Londres).

Nematognatha brevicollis (Champion, 1892): Champion, 1899: 205 (*) Zonitis brevicollis (Champion, 1892): Denier, 1935a: $147\left({ }^{*}\right)$

COMENTARIOS TAXONÓMICOS: La asignación subgenérica de esta especie es tentativa.
DisTRIBUCIÓN GEOGRÁFICA: Especie endémica de México sólo conocida de Puebla.

MÉxICo: Puebla: Izúcar de Matamoros (Champion, 1892).

Nemognatha (Pauronemognatha) insularis Blaisdell, 1925

Nemognatha insularis Blaisdell, 1925: 325 (*)

TERRA TYPICA: "Bernstein's Spring, on Cedros Island". La serie típica consta de tres ejemplares, el holotipo es un macho $\left(\mathrm{n}^{\circ} 1678\right)$ depositado en la colección de la California Academy of Sciences (San Francisco) (fide Blaisdell, 1925).

Zonitis insularis (Blaisdell, 1925): Blackwelder, 1945: 481 (*)

COMENTARIOS TAXONÓMICOS: La asignación subgenérica de esta especie es tentativa.

DISTRIBUCIÓN GEOGRÁFICA: Especie sólo conocida de la Isla Cedros (Blaisdell, 1925).

MÉXICo: Baja California: Isla Cedros (Blaisdell, 1925; Blackwelder, 1945).

Nemognatha (Pauronemognatha) nigripennis LeConte, 1853

Nemognatha nigripennis LeConte, 1853: 347 (*)

TerRa TYPICA: "Santa Fe, New Mexico...". Lectotipo no 4964 designado por Enns (1956) en la colección del Museum of Comparative Zoology (Harvard University, Cambridge, EEUU) (fide Enns, 1956).

Zonitis nigripennis (LeConte, 1853): Denier, 1935a: 149 (*)

DISTRIBUCIÓN GEOGRÁFICA: Esta especie se extiende por el noroeste de México y el suroeste de los EEUU (Enns, 1956; Werner et al., 1966).

MÉXICo: Baja California Sur: Rancho Viejo [Vizcaíno] (Horn, 1894).

Nemognatha (Pauronemognatha) scutellaris LeConte, 1853

Nemognatha scutellaris LeConte, 1853: 347 (*)

TERRA TYPICA: "Sacramento, California....". Lectotipo no 4966 designado por Enns (1956) en la colección del Museum of Comparative Zoology (Harvard University, Cambridge, EEUU) (fide Enns, 1956).

Zonitis scutellaris (LeConte, 1853): Denier, 1935a: 149 (non Motschulsky, 1870, nec Fairmaire, 1892) (*)

COMENTARIOS TAXONÓMICOS: El nombre de Zonitis scutellaris ha sido utilizado por Motschulsky (1870) y Fairmaire (1892) para taxones distintos de N. scutellaris LeConte, 1853.

DiSTRIBUCIÓN GEOGRÁFICA: Esta especie se extiende por el oeste de los EEUU (Enns, 1956) y podría encontrarse en el norte de Baja California ya que Champion (1892) la cita aunque sin más precisión.

Nemognatha (Pauronemognatha) scutellaroides Wellman, 1910

Nemognatha nigripes Champion, 1892: 377 (non Suffrian, 1853) (*) Terra tyPica: "Mexico, Yolos...; Guatemala...”. Cuatro sintipos posiblemente en la colección del British Museum of Natural History.

Nematognatha nigripes (Champion, 1892): Champion, 1899: $205(*)$ 
Nemognatha scutellaroides Wellman, 1910c: 26 (nombre de substitución para Nemognatha nigripes Champion, 1892) (*)

Zonitis scutellaroides (Wellman, 1910): Denier, 1935a: 149 (*)

COMENTARIOS TAXONÓMICOS: La asignación subgenérica de esta especie es tentativa.

DISTRIBUCIÓN GEOGRÁFICA: Especie conocida de México y Guatemala (Champion, 1892; Blackwelder, 1945).

MÉXICo: Oaxaca: Yolos [posiblemente Yolox, ver Selander \& Vaurie, 1962] (Champion, 1892).

Nemognatha (Pauronemognatha) selloa Selander, 1957 Nemognatha selloa Selander, 1957c: $25(*)$

TERRA TYPICA: "9 miles southeast of Tejupan, Oaxaca ". Holotipo en la colección de la Illinois Natural History Survey y paratipos también en la del American Museum of Natural History (EEUU) (fide Selander, 1957c).

COMENTARIOS TAXONÓMICOS: Incluida en el subgénero Pauronemognatha por Selander (1957c).

DisTRIBUCIÓN GEOGRÁFICA: Especie únicamente conocida de México. Citada en los Estados de México, Oaxaca, Puebla y Tlaxcala.

MÉXICo: México: San Juan Teotihuacán (Selander, 1957c). Oaxaca: Asunción Nochixtlán; 9 millas al sureste de Tejupan (Selander, 1957c); Tejupan (Selander, 1957c). Puebla: Tlacotepec (entre Tehuacán y Puebla) (Selander, 1957c). Tlaxcala: 8 millas al oeste de Capulalpam (Selander, 1957c).

Subgénero Pronemognatha Enns, 1956

Pronemognatha Enns, 1956: 714 (*)

ESPECIE TIPO: Nemognatha sparsa LeConte, 1868, por designación original

CONTENIDO Y DISTRIBUCIÓN: El subgénero incluye 6 especies, todas ellas en América del Norte (Enns, 1956).

En México se ha citado la presencia de tres especies, $N$. (P). cantharidis, $N$. $(P)$. selanderi y $N$. $(P)$. zonitoides Además incluimos aquí tentativamente a $N$. $(P)$. flava.

\section{Nemognatha (Pronemognatha) cantharidis MacSwain, 1951}

Nemognatha cantharidis MacSwain, 1951b: 76 (*)

TERRA TYPICA: "Bennet Wash near Parker Dam, San Bernardino Co. California...". Holotipo $n^{\circ}$ 6219, en la colección de la California Academy of Sciences (San Francisco, EEUU) (fide Enns, 1956). La serie típica consta de 16 ejemplares más (fide Enns, 1956).

DISTRIBUCIÓN GEOGRÁFICA: Especie limitada al noreste de México y al suroeste de los EEUU (Enns, 1956; Werner et al., 1966). En México se ha citado en Baja California y Sonora. Es muy posible que las citas de $N$. sparsa de San Quintín y El Chinche, en Baja California (Horn, 1894), correspondan a N. cantharidis.

MÉxICo: Baja California: 5.9 mi. aguas arriba del Arroyo Santa María (MacSwain, 1951b). Sonora: $50 \mathrm{mi}$. al O de Sonoyta (Werner et al., 1966).

Nemognatha (Pronemognatha) flava (Dugès, 1889) Gnathium flavum Dugès, 1889: 107 bis (non Zonitis flava Fabricius, 1775) (*)

TERRA TYPICA: "Jalapa".
Nemognatha flava (Dugès, 1889): Champion, 1892: 380 (*)

Nematognatha flava (Dugès, 1889): Champion, 1899: 205 (*)

Zonitis flava (Dugès, 1889): Denier, 1935a: $148(*)$

COMENTARIOS TAXONÓMICOS: La asignación subgenérica de esta especie es tentativa.

DisTRIBUCIÓN GEOGRÁFICA: Champion (1892) indica la presencia de la especie en México, Guatemala y Costa Rica. Denier (1935a) cita esta especie del Perú por error, pero lo corrige posteriormente (Denier, 1940). 1892).

MÉXICO: Veracruz: Xalapa (Dugès, 1889e; Champion,

\section{Nemognatha (Pronemognatha) selanderi Enns, 1956}

Nemognatha (Pronemognatha) selanderi Enns, 1956: 718 (*)

TERRA TYPICA: "Presidio, Texas...". Holotipo no 62732, en la colección del United States National Museum (fide Enns, 1956). La serie típica consta de 5 ejemplares más (fide Enns, 1956).

COMENTARIOS TAXONÓMICOS: Especie confundida con $N$. zonitoides.

DISTRIBUCIÓN GEOGRÁFICA: Especie distribuida por el norte de México y el suroeste de los EEUU (Enns, 1956). Enns (1956) señala que muchas de las citas de $N$. zonitoides, incluidas parte de las de la descripción original de Dugès (1874a) y las de Selander (1954b), corresponden a $N$. selanderi, por lo que al menos ambas especies se encontrarían en simpatría en Guanajuato.

MÉxICo: Guanajuato: Guanajuato (Dugès, 1874a sub $N$. zonitoides; Enns, 1956). Guerrero: Mexcala (Selander, 1954b sub $N$. zonitoides).

Nemognatha (Pronemognatha) zonitoides Dugès, 1874 Nemognatha zonitoides Dugès, 1874a: $47(*)$

TERRA TYPICA: “ “... cercanías de Guanajuato”. Holotipo en paradero desconocido (fide Enns, 1956).

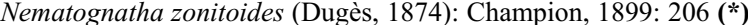
Zonitis zonitoides (Dugès, 1874): Wickham, 1905: $171\left(^{*}\right.$ )

DISTRIBUCIÓN GEOGRÁFICA: Especie distribuida en México y Guatemala (Champion, 1892; Vaurie, 1950; Enns, 1956). En México se ha citado en Chihuahua, Coahuila, Colima, Durango, Guanajuato, Guerrero, Michoacán, Nuevo León, Yucatán y Zacatecas. Enns (1956) señala que muchas de las citas de $N$. zonitoides, incluidas parte de las de la descripción original de Dugès (1874a) y las de Selander (1954b), corresponden a $N$. selanderi. Pero como Enns (1956: 721) no indica qué material mexicano revisó, de momento incluimos aquí todas las demás citas publicadas bajo el nombre de $N$. zonitoides.

MÉXICo: Chihuahua: 2 mi. al O de Matachic (Vaurie, 1950). Coahuila: San Pedro (Champion, 1892; Vaurie, 1950); San Pedro de Colonias (Vaurie, 1950); La Rosa (Vaurie, 1950); Parras (Champion, 1892; Vaurie, 1950); Saltillo (Champion, 1892; Vaurie, 1950). Colima: Tonila (Champion, 1892; Vaurie, 1950). Durango: Villa Lerdo (Champion, 1892; Vaurie, 1950); Encino (Vaurie, 1950); San Lucas (Vaurie, 1950); Yerbanís, Distrito de Cuencamé (Vaurie, 1950); Durango (Vaurie, 1950); 
Palos Colorados (Vaurie, 1950); Otinapa (Vaurie, 1950) Guanajuato: Guanajuato (Dugès, 1874a; Champion, 1892; Vaurie, 1950). Guerrero: Chilpancingo (Champion, 1892; Vaurie, 1950); Rincón (Champion, 1892; Vaurie, 1950). Michoacán: Morelia (Dugès, 1889e; Champion, 1892; Vaurie, 1950). Nuevo León: Monterrey (Champion, 1892; Vaurie, 1950). Yucatán: Temax (Champion, 1892; Vaurie, 1950). Zacatecas: Fresnillo (Vaurie, 1950).

Género Pseudozonitis Dillon, 1952

Pseudozonitis Dillon, 1952: 342 (*)

ESPECIE TIPO: Zonitis longicornis Horn, 1870, por designación original.

CONTENIDO Y DISTRIBUCIÓN: El género incluye 19 especies distribuidas en América del Norte, desde Panamá al sur de los Estados Unidos, incluidas las Antillas y en América del Sur (Pinto \& Bologna, 1999).

En México hay citas de $P$. martini, $P$. vaurieae y $P$. megalops (Enns, 1956; Selander \& Bouseman, 1960), pero posiblemente existan muchas otras especies que se encuentran cerca de la frontera con EEUU. Entre esas destacan por su proximidad: P. arizonica (Van Dyke, 1929), P. brevis Enns, 1956, P. labialis Enns, 1956, P. pallida Dillon, 1952, P. stroudi Enns, 1956, P. vigilans (Fall, 1907) y P. vittipennis (Horn, 1875).

Pseudozonitis martini (Fall, 1907)

Zonitis martini Fall in Fall et Cockerell, 1907: 257 (*)

Terra typicA: "Engle, New Mexico". Holotipo no 24310, en la colección del Museum of Comparative Zoology (Harvard University, Cambridge, EEUU) (fide Enns, 1956).

Pseudozonitis martini (Fall, 1907): Dillon, 1952: $342\left(^{*}\right)$

COMENTARIOS TAXONÓMICOS: Enns (1956) señala que P. castaneis Dillon, 1952 y P. vogti Dillon, 1952 seguramente sean variantes de color de $P$. martini.

DisTRIBUCIÓN GEOGRÁFICA: Especie, al parecer, distribuida por el norte de México y el suroeste de los EEUU (Enns, 1956), aunque Werner et al. (1966) no recogen su presencia en México.

MÉXICO: sin más precisión (Enns, 1956).

Pseudozonitis megalops (Champion, 1892)

Zonitis megalops Champion, 1892: 386 (*)

Terra TYPICA: "Guatemala, Volcan de Atlitlan 3000 feet".

Holotipo en la colección del British Museum of Natural History (fide Enns, 1956).

Pseudozonitis megalops (Champion, 1892): Selander, 1954b: 92 (*)

DisTRIBUCIÓN GEOGRÁFICA: Según Enns (1956), esta especie estaría distribuida por el sur de México, Guatemala y Nicaragua. Pero el propio Enns (1956) señala que las citas de $P$. megalops en los trabajos de Vaurie (1950) y de Selander (1954b) corresponden a P. vaurie$a e$, por lo que no existirían citas concretas de $P$. megalops en México a excepción de un registro "tentatively identified as P. megalops" (Selander \& Bouseman, 1960) de Oaxaca.

MÉXICo: Oaxaca: Oaxaca (Selander \& Bouseman, 1960).
Pseudozonitis vaurieae Enns, 1956

Pseudozonitis vaurieae Enns, 1956: 871 (*)

TERRA TYPICA: "Pedricena, Durango, Mexico...". Holotipo en la colección del American Museum of Natural History (fide Enns, 1956). La serie típica consta de numerosos ejemplares más (fide Enns, 1956).

COMENTARIOS TAXONÓMICOS: Incluida en la denominación de Z. megalops (Vaurie, 1950; Selander, 1954b) hasta el trabajo de Enns (1956).

DISTRIBUCIÓN GEOGRÁFICA: Especie distribuida por el oeste y norte de México y el suroeste de los EEUU (Enns, 1956). En México se ha citado en Baja California, Baja California Sur, Chihuahua, Coahuila, Durango, Guerrero, Nayarit, Nuevo León, Sinaloa y Sonora. Enns (1956) señala que las citas de $P$. megalops en los trabajos de Vaurie (1950) y de Selander (1954b) corresponden a esta especie.

MÉxICo: Baja California: Mesquital (Enns, 1956); Santo Domingo (Enns, 1956); San Felipe (Enns, 1956). Baja California Sur: 6 mi. al N de Triunfo (Enns, 1956); 20 mi. al $\mathrm{N}$ de Comondú (Enns, 1956); Venancio (Enns, 1956). Chihuahua: Delicias (Vaurie, 1950 sub P. megalops; Enns, 1956). Coahuila: norte de Coahuila (Werner et al., 1966). Durango: Pedricena (Vaurie, 1950 sub P. megalops; Enns, 1956); 35 mi. al S de El Entronque (Selander, 1954b sub $P$. megalops; Enns, 1956). Guerrero: Acapulco (Enns, 1956). Nayarit: Acaponeta (Enns, 1956). Nuevo León: Vallecillo (Enns, 1956). Sinaloa: Los Mochis (Enns, 1956). Sonora: 42 mi. al S de Hermosillo (Enns, 1956); Guaymas (Enns, 1956).

\section{Género Rhyphonemognatha Enns, 1956}

Rhyphonemognatha Enns, 1956: 795 (*)

ESPECIE TIPO: Zonitis sanguinicollis Champion, 1892, por designación original.

CONTENIDO Y DISTRIBUCIÓN: El género incluye cuatro especies distribuidas en América del Norte, desde Panamá al sur de los Estados Unidos y al parecer presente también en América del Sur (Pinto \& Bologna, 1999). Las cuatro especies del género reconocidas actualmente se encuentran en México.

Rhyphonemognatha flavicollis (Dugès, 1881)

Zonitis flavicollis Dugès, 1881: 147 (*)

TERRA TYPICA: "Guanajuato". Holotipo en paradero desconocido.

Rhyphonemognatha flavicollis (Dugès, 1881): Enns, 1956: 796 (*)

DiSTRIBUCIÓN GEOGRÁFICA: Especie endémica de México (Enns, 1956), sólo citada en Guanajuato.

MÉxICo: Guanajuato: Guanajuato (Dugès, 1881; Dugès, 1889e; Champion, 1892).

Rhyphonemognatha rufa (LeConte, 1854)

Zonitis rufa LeConte, 1854c: 85 (*)

TERRA TYPICA: "Frontera (Rio Grande)"; New Mexico en el holotipo (fide Enns, 1956). Holotipo no 4976, en la colección del Museum of Comparative Zoology (Harvard University, Cambridge, EEUU), pero podría estar mal ya que no coincide con la localidad original (fide Enns, 1956).

Zonitis rubra Dugès, 1870: 166 (*)

TERRA TYPICA: “...cerca de Guanajuato”. Holotipo perdido (fide Enns, 1956) 
Nemognatha rufa (LeConte, 1854): Dillon, 1952: 341 (*)

Rhyphonemognatha rufa (LeConte, 1854): Enns, 1956: 796 (*)

COMENTARIOS TAXONÓMICOS: Beauregard (1890) mantiene como especies diferentes a Z. rufa y Z. rubra, e indica como sinónimo de esta última a $Z$. coccinea Klug (in litt.) un nombre no disponible. Werner et al. (1966) sugieren implícitamente que la taxonomía de los ejemplares del sur de México debe ser revisada.

DISTRIBUCIÓN GEOGRÁFICA: Especie distribuida por el norte de México y centro de los EEUU (Enns, 1956). Citada en el Distrito Federal, Guanajuato y Oaxaca.

MÉXICo: Distrito Federal: Ciudad de México (Champion, 1892). Guanajuato: Guanajuato (Dugès, 1870; Dugès, 1889e sub Z. rubra; Champion, 1892); Tupátaro (Dugès, 1889e sub Z. rubra; Champion, 1892). Oaxaca: La Parada (Champion, 1892).

Rhyphonemognatha sanguinicollis (Champion, 1892) Zonitis sanguinicollis Champion, 1892: $384(*)$

Terra typica: "MeXico, Cordova, Teapa in Tabasco; Guatemala, Coban, Senahu, Dueñas, Capetillo; NicARAGuA, Chontales; Panama, Chiriqui". Sintipos en la colección del British Museum of Natural History (Londres) (fide Enns, 1956). Enns (1956) indica que el "tipo" (ejemplar figurado) es de la localidad de "Dueñas, Guatemala" pero no indica si designó lectotipo formalmente.

Rhyphonemognatha sanguinicollis (Champion, 1892): Enns, 1956 $796(*)$

DistribuCIÓN GEOGRÁfICA: Especie distribuida desde México hasta Panamá, con citas en Guatemala y Nicaragua (Enns, 1956; Maes \& Huether, 2007). En México se ha citado en Veracruz y Tabasco.

MÉXICo: Veracruz: Córdoba (Champion, 1892). Tabasco: Teapa (Champion, 1892).

\section{Rhyphonemognatha tenebrosa (Champion, 1892)}

Zonitis tenebrosa Champion, 1892: 383 (*)

TERRA TYPICA: "MeXICO, Tacambaro in Michoacan". Tipo en la colección del British Museum of Natural History (Londres).

Rhyphonemognatha tenebrosa (Champion, 1892): Enns, 1956: 796 (*)

DISTRIBUCIÓN GEOGRÁFICA: Especie endémica de México (Enns, 1956) sólo conocida de la localidad típica en Michoacán.

MÉXICO: Michoacán: Tacámbaro (Champion, 1892).

Género Zonitis Fabricius, 1775

Zonitis Fabricius, 1775: 126 (*)

ESPECIE TIPO: Zonitis flava Fabricius, 1775, por designación subsecuente de Selander (1987b).

CONTENIDO Y DISTRIBUCIÓN: El género incluye 163 especies distribuidas por todo el Planeta (Pinto \& Bologna, 1999). Las 36 especies americanas se agrupan en dos subgéneros, Neozonitis y Parazonitis, mientras que las del Viejo Mundo se incluyen en el subgénero nominal. El género podría no ser monofilético, en particular con respecto a Pseudozonitis y a Zonitoschema (Pinto \& Bologna, 1999). Aksentjev (1988: 577) elevó Neozonitis a la categoría genérica, incluyendo a Parazonitis como sub- género, pero Bologna (1991), Pinto \& Bologna (1999) y Pinto (2001) lo consideran a nivel subgenérico.

Vaurie (1950) cita algunos ejemplares prodecedentes de localidades mexicanas que no asigna a ninguna especie en concreto y que incluye bajo el epígrafe de especies desconocidas de Zonitis. Estos ejemplares son: Sp.1: Chihuahua: Samalayuca (Vaurie, 1950). Sp.2: Durango: Palos Colorados (Vaurie, 1950); Coyotes (Vaurie, 1950). Sp.3: Chihuahua: Balleza (Vaurie, 1950). Durango: Encino (Vaurie, 1950).

Las especies presentes en México cuya adscripción subgenérica no ha sido revisada y que incluso podrían pertenecer a otros géneros, se indican a continuación.

Zonitis (?) nigromaculata Dugès, 1889

Zonitis nigromaculata Dugès, 1889e: 111 bis (*) Terra typica: "Atoyac".

COMENTARIOS TAXONÓMICOS: De acuerdo con la descripción original, Zonitis bilineatithorax Pic, 1914, de Guatemala, podría ser una variante de coloración de $Z$. nigromaculata.

DisTRIBUCIÓN GEOGRÁFICA: Especie hasta el momento exclusiva de México (Champion, 1892), citada únicamente en Veracruz.

MÉXICo: Veracruz: Atoyac (Dugès, 1889e; Champion, 1892); Tuxtla (Champion, 1892).

Zonitis (?) xanthochroa Wellman, 1910

Zonitis fulva Dugès, 1889e: 111 bis (non Zonitis fulva Rossi, 1792) (*) TERRA TYPICA: "Atoyac".

Zonitis xanthochroa Wellman, 1910c: 26 (nombre de substitución para Zonitis fulva Dugès, 1889) (*)

DISTRIBUCIÓN GEOGRÁFICA: Especie citada en México y Guatemala (Champion, 1892), con registros en Veracruz.

MÉxICo: Veracruz: Atoyac (Dugès, 1889e sub Z. fulva; Champion, 1892); Córdoba (Champion, 1892); Cerro de Plumas [Cerro de Palmas] (Champion, 1892).

Subgénero Neozonitis Enns, 1956

Neozonitis Enns, 1956: 800 (*)

ESPECIE TIPO: Zonitis bilineata Say, 1817, por designación original

CONTENIDO Y DISTRIBUCIÓN: El subgénero incluye nueve especies en América al norte de México (Enns, 1956; Pinto, 2001). En México se han citado tres especies, Z. (N.). florhi, Z. (N.) minutissima y Z. (N.). atripennis y aquí atribuimos tentativamente al subgénero Neozonitis una cuarta, Z. ploribunda. Además, podrían encontrarse varias especies más que han sido citadas cerca de la frontera con los EEUU (Werner et al., 1966).

Zonitis (Neozonitis) atripennis (Say, 1824)

Zonitis (Neozonitis) atripennis terminalis Enns, 1956

Zonitis (Neozonitis) atripennis terminalis Enns, 1956: 811 (*)

TERRA TYPICA: "California". Holotipo en la colección del Museum of Comparative Zoology (Harvard University, Cambridge, EEUU) (fide Enns, 1956). 
COMENTARIOS TAXONÓMICOS: Enns (1956) divide la especie en tres subespecies parapátricas $Z$. a. atripennis (Say, 1824), Z. a. flavida (LeConte, 1853) y Z. a. terminalis Enns, 1956.

DisTRIBUCIÓN GEOGRÁFICA: Especie ampliamente distribuida por los Estados Unidos, subdividida en tres razas geográficas, una de las cuales, Z. a. terminalis, ha sido citada en la Península de Baja California (Enns, 1956; Werner et al., 1966).

Z. (N.) atripennis terminalis: MÉXICo: Baja California Sur: sur de Baja California (Werner et al., 1966).

Zonitis (Neozonitis) florhi Dugès, 1889

Zonitis florhi Dugès, 1889e: 110 (*)

TerRa TYPICA: "Vera Cruz".

COMENTARIOS TAXONÓMICOS: Incluida tentativamente en el subgénero Neozonitis por Werner et al. (1966) sin que su estatus haya sido revisado. Dugès (1886a: 582) utilizó previamente el nombre Zonitis florhi sin acompañarlo de una descripción. Beauregard (1890: 471) incluye el error tipográfico Zonitis flohri.

DISTRIBUCIÓN GEOGRÁFICA: Especie conocida del Estado de Veracruz, con un único registro fuera del Estado, en Arizona (EEUU) (Werner et al., 1966).

MÉXICO: Veracruz: Xalapa (Champion, 1892); Orizaba (Champion, 1892); Veracruz (Dugès, 1889e; Champion, 1892).

Zonitis (Neozonitis) minutissima Pinto, 2001

Zonitis (Neozonitis) minutissima Pinto, 2001: 321 (*)

TerRa tYPICA: "United States: Arizona: Picacho Pass (Pinal

Co.)...". Holotipo en la colección de la California Academy of Sciencies (San Francisco, EEUU) (fide Pinto, 2001).

COMENTARIOS TAXONÓMICOS: Incluida tentativamente en el subgénero Neozonitis por Pinto (2001).

DisTRIBUCIÓN GEOGRÁFICA: Especie conocida únicamente de Baja California Sur y Arizona (EEUU) (Pinto, 2001).

MÉXICo: Baja California Sur: 33.8 mi. al NNO de Loreto (Pinto, 2001).

Zonitis (Neozonitis) ploribunda Wellman, 1910

Zonitis atra Dugès, 1881: 146 (non Zonitis atrum Schwartz, 1808) (*) TERRA TYPICA: “.... en un cerro de Guanajuato”. Holotipo en paradero desconocido, posiblemente perdido.

Zonitis ploribunda Wellman, 1910c: 25 (nombre de substitución para Zonitis atra Dugès, 1881) (*)

COMENTARIOS TAXONÓMICOS: La asignación subgenérica de esta especie es tentativa. Dugès describió en 1881 Zonitis atra y en 1889 Gnathium atrum. Champion (1892) considera ambas como especies distintas, la primera en el género Zonitis, la segunda en Nemognatha, mientras que Selander (1954b) incluye ambas en el género Zonitis.

DisTRIBUCIÓN GEOGRÁFICA: Especie endémica de México, citada en Guanajuato.
MÉXICO: Guanajuato: Guanajuato (Dugès, $1881 \mathrm{sub} Z$. atra); León (Dugès, 1889e sub Z. atra; Champion, 1892 sub Z. atra).

Subgénero Parazonitis Enns, 1956

Parazonitis Enns, 1956: 819 (*)

ESPECIE TIPO: Nemognatha vittigera LeConte, 1853, por designación original.

CONTENIDO Y DISTRIBUCIÓN: El subgénero incluye 7 especies en América al norte de México (Enns, 1956).

En México se han citado dos especies, $Z$. (P.). sayi y $Z$. (P.). tarasca e incluimos tentativamente a $Z$. dugesi. Además, podrían encontrarse casi todas las demás especies que han sido citadas cerca de la frontera con EEUU como Z. (P.). cribricollis (LeConte, 1853), Z. (P.). dunniana Casey, 1891, Z. (P.). perforata Casey, 1891 y $Z$. (P.). vittigera propinqua MacSwain, 1951.

Zonitis (Parazonitis) dugesi Selander, 1954

Grathium atrum Dugès, 1889e: 113 (y error tipográfico en el género) (*) TERRA TYPICA: "Tupátaro". Holotipo en paradero desconocido. Nemognatha atra (Dugès, 1889): Champion, 1892: $380(*)$

Nematognatha atra (Dugès, 1889): Champion, 1899: $205\left(^{*}\right)$

Zonitis atra (Dugès, 1889): Denier, 1935a: $147\left(^{*}\right)$

Zonitis dugesi Selander, 1954b: 95 (nombre de substitución para Gnathium atrum Dugès, 1889) (*)

COMENTARIOS TAXONÓMICOS: La asignación subgenérica de esta especie es tentativa. Dugès describió en 1881 Zonitis atra y en 1889 Gnathium atrum. Champion (1892) considera ambas como especies distintas, la primera en el género Zonitis, la segunda en Nemognatha. Sin embargo Selander (1954b), considera que ambas especies pertenecen al género Zonitis. Mientras la situación no se resuelva con un estudio taxonómico comparativo, mantenemos las dos especies de Dugès en Zonitis como propuso Selander (1954b).

DistriBuCión GEOGRÁFICA: Especie endémica de México (Selander, 1954b), aunque existe una cita de Dillon (1952) de Texas que Selander (1954b) considera un error de identificación. Citada en Guanajuato y Guerrero.

MÉXICO: Guanajuato: Tupátaro (Dugès, 1889e; Champion, 1892; Selander, 1954b). Guerrero: Mexcala (Selander, 1954b sub Zonitis dugesi).

\section{Zonitis (Parazonitis) sayi Wickham, 1905}

Nemognatha immaculata Say, 1817: 22 (non Apalus immaculatus Olivier, $1789=$ Zonitis immaculata $)(*)$

TERRA TYPICA: “...plains of the Missouri”. Tipo original posiblemente perdido.

Zonitis immaculata (Say, 1817): Casey, 1891: 170 (*)

Zonitis sayi Wickham, 1905: 171 (nombre de substitución para Nemognatha immaculata Say, 1817) (*)

Nemognatha sayi (Wickham, 1905): Leng, 1920: 160 (*)

COMENTARIOS TAXONÓMICOS: Say (1817) ya indica que podría corresponder en realidad al género Zonitis.

DisTRIBUCIÓN GEOGRÁFICA: Especie ampliamente distribuida desde el norte de México hasta el sur del 
Canadá, con numerosas citas en el centro de los EEUU (Enns, 1956). En México se ha citado en Chihuahua.

MÉxICo: Chihuahua: Santa Clara (Champion, 1892; Vaurie, 1950 sub Zonitis immaculata).

\section{Zonitis (Parazonitis) tarasca (Dugès, 1889)}

Zonitis (Parazonitis) tarasca tarasca (Dugès, 1889)

Nemognatha tarasca Dugès, 1889e: 112 (*)

TERRA TYPICA: "Morelia" originalmente; "Palos Colorados, Durango, Mexico" si la designación de neotipo en la colección del American Museum of Natural History (Enns, 1956) es correcta. Vázquez \& Zaragoza (1979) indican como tipo el ejemplar IBUNAM 032, de la colección del Instituto de Biología de la Universidad Nacional Autónoma de México.

Nematognatha tarasca (Dugès, 1889): Champion, 1899: 205 (*)

Zonitis tarasca (Dugès, 1889e): Denier, 1935a: $150(*)$

Zonitis (Parazonitis) tarasca borealis Enns, 1956

Zonitis (Parazonitis) tarasca borealis Enns, 1956: 842 (*)

TERRA TYPICA: "Cochise Stronghold, Dragoon Mountains, Arizona”. Holotipo en la colección del Museum of Comparative Zoology (Harvard University, Cambridge, EEUU) (fide Enns, 1956). La serie típica consta de numerosos ejemplares más, incluyendo uno de Durango (fide Enns, 1956).

DistribuCiÓN GEOGRÁFICA: Especie ampliamente distribuida desde Panamá hasta el sur del Canadá, con numerosas citas en el centro de los EEUU (Champion, 1892; Enns, 1956; Werner et al., 1966; Maes \& Huether, 2007). En México se ha citado en Coahuila, Durango, Guerrero, Michoacán, Morelos, Nayarit, Oaxaca, Tabasco, Veracruz y Yucatán. Según Enns (1956) la cita de Selander (1954b) de Z. punctipennis (LeConte, 1880) en Durango corresponde a Z. tarasca borealis. Enns (1956) indica que los ejemplares de San Gerónimo (Guatemala) de Champion (1892) podrían corresponder a una especie distinta. Las citas de Vaurie (1950) como "species unknown" de Durango y Yucatán: Palos Colorados; Otinapa; Nombre de Dios; 6 mi. al NE de El Salto; Temax, posiblemente corresponden a esta especie.

Z. (P.) tarasca tarasca: MÉXICO: Coahuila: Saltillo (Enns, 1956). Durango: Palos Colorados (Enns, 1956); Canutillo (Enns, 1956); El Salto (Enns, 1956); Otinapa (Enns, 1956). Guerrero: Chilpancingo (Champion, 1892; Enns, 1956). Michoacán: Morelia (Dugès, 1889e; Champion, 1892; Enns, 1956; Vázquez \& Zaragoza, 1979). Morelos: Alpuyeca (Enns, 1956). Nayarit: Navarrete (Enns, 1956); Tepic (Enns, 1956); Tuxpan (Enns, 1956). Oaxaca: Valerio Trujano (Enns, 1956); Etla (Champion, 1892; Enns, 1956). Tabasco: Jalapa (Enns, 1956). Veracruz: Orizaba (Enns, 1956); Tuxtla (Champion, 1892; Enns, 1956); Veracruz (Enns, 1956). Yucatán: Temax (Enns, 1956).

Z. (P.) tarasca borealis: MÉXICo: Durango: $10 \mathrm{mi}$. al N de El Entronque (Enns, 1956; Selander, 1954b sub Z. punctipennis).

Subfamilia Tetraonycinae Böving et Craighead, 1930 Tetraonycidae Böving \& Craighead, 1930: 59 (*)

CONTENIDO Y DISTRIBUCIÓN: La subfamilia incluye unas 103 especies, distribuidas en cuatro géneros,
Meloetyphlus Waterhouse, 1872, monotípico; Opiomeloe Selander, 1985, monotípico; Tetraolytta Pic, 1919, monotípico y Tetraonyx, en el que se integran el resto de las especies (Selander, 1965b, 1985a; Pinto \& Bologna, 1999; Bologna \& Pinto, 2007). La distribución geográfica de la subfamilia se limita al continente americano (Pinto \& Bologna, 1999). Todos los representantes de la subfamilia se incluyen en la tribu Tetraonycini.

Tribu Tetraonycini Böving et Craighead, 1930

Tetraonycidae Böving \& Craighead, 1930: 59 (*)

Contenido Y Distribución: Incluye a todos los representantes de la subfamilia (Pinto \& Bologna, 1999). En México únicamente se conocen representantes del género Tetraonyx.

\section{Género Tetraonyx Latreille, 1805}

Tetraonyx Latreille, 1805: 204 (*)

ESPECIE TIPO: Tetraonyx octomaculatus Latreille, 1805, por monotipia.

CONTENIDO Y DISTRIBUCIÓN: El género está representado por 101 especies, que se extienden por la mayor parte de América del Sur y desde Panamá hasta el sur de los Estados Unidos y las Antillas, en América del Norte (Pinto \& Bologna, 1999). La taxonomía del género está sin revisar, en particular la de la vasta mayoría de especies neotropicales (Pinto \& Bologna, 1999). Únicamente se han separado dos subgéneros, Paratetraonyx Kaszab, 1959, monotípico y distribuido en América del Sur y Tetraonyx, ampliamente extendido por todo el continente americano. Pinto \& Bologna (1999) sugieren que Paratetraonyx podría ser un sinónimo de Tetraolytta Pic, 1919. Las especies mexicanas se integran en el subgénero nominal.

El género gramatical del nombre Tetraonyx es masculino tal como se utilizó en los trabajos de Latreille (1805), Haag-Rutenberg (1879), Dugès (1881, 1889e), Denier (1935a) y Selander \& Martínez (1984).

\section{Subgénero Tetraonyx Latreille, 1805}

Tetraonyx Latreille, 1805: 204 (*)

ESPECIE TIPO: Tetraonyx octomaculatus Latreille, 1805, por monotipia.

Iodema Pascoe, 1862: 57 (*)

ESPECIE TIPO: Iodema clarkii Pascoe, 1862 (= Tetraonyx violaceipennis Lucas in Laporte de Castelnau, 1859), por monotipia.

Jodema Pascoe, 1862: Gemminger et Harold, 1870: 2146 (enmienda injustificada) (*)

COMENTARIOS TAXONÓMICOS: En la obra de Chevrolat (1858: 210) se desliza el error tipográfrico Tetraomyx.

CONTENIDO Y DISTRIBUCIÓN: El subgénero está representado por 100 especies, con la misma distribución que la del género (Pinto \& Bologna, 1999). En México se han citado 13 especies, aunque existen dos especies problemáticas descritas por Haag-Rutenberg (1879): $T$. decipiens y $T$. angulicollis, que no se han vuelto a citar 
desde su descripción y una tercera descrita por Klug (1825) del Brasil, T. bimaculatus, que fue citada por Champion (1892) en México y que posiblemente deba excluirse del catálogo de México. Denier (1935a) cita $T$. bilineatus Haag-Rutenberg, 1879 en México, pero se trata de un error que corrige posteriormente (Denier, 1840), ya que la cita correspondía a $T$. bipartitus. Champion (1892) indica que T. quadrimaculatus (Fabricius, 1792), de las Antillas y de los Estados Unidos, podría encontrarse también en México.

Tetraonyx (Tetraonyx) angulicollis Haag-Rutenberg, 1879 Tetraonyx angulicollis Haag-Rutenberg, 1879: 270 (*)

TERRA TYPICA: "Mexico?". Tipo posiblemente en la colección HaagRutenberg, del Zoologische Staatssammlung (Munich).

Comentarios taXonÓmicos: Especie descrita con dudas de México (Haag-Rutenberg, 1879; Champion, 1892; Borchmann, 1917; Selander, 1983a), sin que se haya vuelto a citar desde su descubrimiento.

Tetraonyx (Tetraonyx) batesi Haag-Rutenberg, 1879 Tetraonyx batesi Haag-Rutenberg, 1879: 267 (*)

TERRA TYPICA: "Juquida [sic] (Oaxaca)". Tipos en la Colección Boucard y Colección Sallé (fide Haag-Rutenberg, 1879).

DisTRIBUCIÓN GEOGRÁFICA: Especie distribuida en México y Guatemala (Haag-Rutenberg, 1879; Champion, 1892; Selander, 1983a). Citada en los estados de Oaxaca y Tabasco.

MÉxICO: Oaxaca: Juquila (Haag-Rutenberg, 1879; Champion, 1892; Selander, 1983a); Oaxaca (Champion, 1892); Tehuantepec (Champion, 1892; Selander, 1983a); Tepansacualco (Tepamacoalco) (Champion, 1892; Selander, 1983a). Tabasco: Teapa (Champion, 1892; Selander, 1983a)

\section{Tetraonyx (Tetraonyx) bimaculatus (Klug, 1825)}

Lytta bimaculata Klug, 1825: 448 (*)

TERRA TYPICA: no indicada, aunque por el título del trabajo correspondería al Brasil. Holotipo perdido (fide Denier, 1935b).

Tetraonyx bimaculatus (Klug, 1825): Le Peletier et Audinet-Serville, 1825: 595

COMENTARIOS TAXONÓMICOS: Especie, considerada por Beauregard (1890), Wellman (1910), Borchmann (1917) y Denier (1935b) como una variedad de T. quadrimaculatus (Fabricius, 1792), que Selander \& Bouseman (1960) restituyen al estatus específico. Champion (1892) indica a Lacordaire como el autor del cambio de género.

DisTRIBUCIÓN GEOGRÁFICA: Especie distribuida en el Brasil y la Argentina (Selander, 1983a; Selander \& Martínez, 1984), citada en México sin más precisión, una única vez (Champion, 1892). Posiblemente deba excluirse del catálogo de especies mexicanas.

Tetraonyx (Tetraonyx) bipartitus Haag-Rutenberg, 1879 Tetraonyx bipartitus Haag-Rutenberg, 1879: 267 (*)

TERRA TYPICA: "Mexico (Mirador)".
DisTRIBUCIÓN GEOGRÁFICA: Especie distribuida desde México hasta Nicaragua (Selander, 1983a). Citada en Veracruz.

MÉXICo: Veracruz: Almolonga (Dugès, 1889e; Champion, 1892; Selander, 1983a); Hacienda El Mirador (HaagRutenberg, 1879; Champion, 1892; Selander, 1983a).

Tetraonyx (Tetraonyx) decipiens Haag-Rutenberg, 1879 Tetraonyx decipiens Haag-Rutenberg, 1879: 269 (*)

TERRA TYPICA: "Mexico". Holotipo posiblemente en la colección Haag del Zoologische Staatssammlung (Munich).

DisTRIBUCIÓN GEOGRÁFICA: Especie descrita de México sin más precisión (Haag-Rutenberg, 1879; Selander, 1983a). Las únicas citas posteriores, Brèthes (1915), del Paraguay (Puerto Cantera), son consideradas erróneas por Denier (1935a).

\section{Tetraonyx (Tetraonyx) dubiosus Horn, 1894}

Tetraonyx dubiosa Horn, 1894: 440 (*)

TERRA TYPICA: “El Chinche, San Julio". Tipos posiblemente en la colección Horn de la Academy of Natural Sciences de Philadelphia (EEUU).

Distribución GEOGRÁfiCA: Sólo conocido de la localidad típica en la Península de Baja California (Selander, 1983a).

MÉXICo: Baja California Sur: El Chinche [Cerro de la Chinche], San Julio (Horn, 1894; Selander, 1983a).

Tetraonyx (Tetraonyx) femoralis Dugès, 1869

Tetraonyx femoralis Dugès, 1869a: 104 (*)

TERRA TYPICA: "mineral del Cedro, y ... orillas de Guanajuato".

COMENTARIOS TAXONÓMICOS: Beauregard (1890), Champion (1892) y Borchmann (1917) consideran a $T$. femoralis como una variedad de $T$. frontalis y Vaurie (1950) lo incluye en su sinonimia, aunque Werner et al. (1966) y Selander (1983a) no comparten su criterio.

DisTRIBUCIÓN GEOGRÁFICA: Se distribuye por el centro y norte de México y el sur de los EEUU (Selander, 1983a). En México se ha citado en los estados de Distrito Federal, Durango, Guanajuato, Jalisco, Michoacán, Sonora y Veracruz.

MÉxICo: Distrito Federal: Ciudad de México (Champion, 1892 sub T. frontalis; Vaurie, 1950 sub T. frontalis; Selander, 1983a); Santa Fé (Vaurie, 1950 sub T. frontalis; Selander, 1983a). Durango: Yerbanís, Distrito de Cuencamé (Vaurie, 1950 sub T. frontalis; Selander, 1983a). Guanajuato: Guanajuato (Dugès, 1869a; Champion, 1892 sub T. frontalis; Vaurie, 1950 sub T. frontalis; Selander, 1983a); Mineral del Cedro (Dugès, 1869a; Selander, 1983a). Jalisco: sin precisar (Werner et al., 1966). Michoacán: Morelia (Dugès, 1889e; Champion, 1892 sub T. frontalis; Vaurie, 1950 sub T. frontalis; Selander, 1983a); Tacámbaro (Champion, 1892 sub T. frontalis; Vaurie, 1950 sub T. frontalis; Selander, 1983a); Uruápan (Dugès, 1889e; Champion, 1892 sub T. frontalis; Vaurie, 1950 sub T. frontalis; Selander, 1983a). Sonora: Northern Sonora (Champion, 1892 sub T. frontalis); $6 \mathrm{mi}$. al NO de Cananea (Werner et al., 1966; Selander, 1983a). Veracruz: Xalapa (Champion, 1892 sub T. frontalis; Selander, 1983a). 
Tetraonyx (Tetraonyx) frontalis Chevrolat, 1833

Tetraonyx frontalis Chevrolat, 1833: fasc. 1, $\mathrm{n}^{\circ} 14$ (sin paginar) (*) TERRA TYPICA: "Orixaba".

COMENTARIOS TAXONÓMICOS: Beauregard (1890) incluye como sinónimo de $T$. frontalis el nombre no disponible Tetraonyx bicolor Klug in Dejean, 1837: 248. Champion (1892) incluye en la sinonimia el nombre no disponible Tetraonyx cinnamomea Sturm, 1843: 175.

DISTRIBUCIÓN GEOGRÁFICA: Su distribución geográfica incluye México, Guatemala, El Salvador y el sur de los EEUU (Selander, 1983a). En México se ha citado en el Distrito Federal, Guerrero, Oaxaca, Puebla y Veracruz.

MÉXICo: Distrito Federal: Ciudad de México (Champion, 1892). Guerrero: Amula (Champion, 1892; Vaurie, 1950; Selander, 1983a); Mochitlán (Champion, 1892; Vaurie, 1950); Xautipa (Champion, 1892; Vaurie, 1950). Oaxaca: Juquila (Champion, 1892; Vaurie, 1950); Oaxaca (Champion, 1892; Vaurie, 1950); La Parada (Champion, 1892). Puebla: Puebla (Champion, 1892; Vaurie, 1950; Selander, 1983a). Veracruz: Xalapa (Champion, 1892; Vaurie, 1950; Selander, 1983a) Orizaba (Chevrolat, 1833; Dugès, 1869a; Champion, 1892; Vaurie, 1950; Selander, 1983a); Córdoba (Champion, 1892; Vaurie, 1950); Toxpam [Tuxpan] (Champion, 1892; Vaurie, 1950); Veracruz (Dugès, 1889e; Champion, 1892).

Tetraonyx (Tetraonyx) fulvus LeConte, 1853

Tetraonyx fulva LeConte, 1853: 344 (*)

TERRA TYPICA: "New Mexico..."

Tetraonyx rufus Dugès, 1869a: $105\left({ }^{*}\right)$

TERRA TYPICA: “cerros de Guanajuato...”. Vázquez \& Zaragoza (1979) indican como tipo el ejemplar IBUNAM 003 de la colección del Instituto de Biología de la Universidad Nacional Autónoma de México.

COMENTARIOS TAXONÓMICOS: Champion (1892) incluye el nombre no disponible Tetraonyx nigritarsis Sturm, 1843: 175, en la sinonimia de T. fulvus.

DISTRIBUCIÓN GEOGRÁFICA: Su distribución geográfica incluye el centro y norte de México y el sur de los EEUU (Selander, 1983a). En México se ha citato en los estados de Chihuahua, Coahuila, Durango y Guanajuato.

MÉxICo: Chihuahua: 2 mi. al NE de Camargo (Selander, 1954b; Selander, 1983a); 5 mi. al N de Delicias (Selander, 1954b; Selander, 1983a); Delicias (Vaurie, 1950; Selander, 1983a). Coahuila: Parras (Champion, 1892; Vaurie, 1950; Selander, 1983a). Durango: Villa Lerdo (Champion, 1892; Vaurie, 1950; Selander, 1983a); Yerbanís, Distrito de Cuencamé (Vaurie, 1950; Selander, 1983a). Guanajuato: Cerros de Guanajuato (Dugès, 1869a sub T. rufus; Selander, 1983a); Guanajuato (Dugès, 1889e; Champion, 1892; Vázquez \& Zaragoza, 1979); Irapuato (Champion, 1892; Vaurie, 1950; Selander, 1983a); León (Dugès, 1889e; Vaurie, 1950; Selander, 1983a).

Tetraonyx (Tetraonyx) maculatus Laporte de Castelnau, 1840

Tetraonyx maculatus Laporte de Castelnau, 1840: 277

TERRA TYPICA: Brasil (fide Selander, 1983a).
Tetraonyx maculatus var. innotatipennis Pic, 1915b: 14 (*) TERRA TYPICA: "Caracas".

COMENTARIOS TAXONÓMICOS: Haag-Rutenberg (1879), Beauregard (1890) y Champion (1892), indican en la sinonimia de esta especie el nombre no disponible $T$. maculicollis Deyr.

DiSTRIBUCIÓN GEOGRÁFICA: Especie distribuida desde Brasil hasta México, con citas además en Guatemala, Nicaragua y Venezuela (Selander, 1983a). Citada en Veracruz.

MÉXICo: Veracruz: Xalapa (Champion, 1892; Selander, 1983a); Veracruz (Champion, 1892; Selander, 1983a).

Tetraonyx (Tetraonyx) octomaculatus Latreille, 1805 Tetraonyx octomaculatus Latreille, 1805 in Humboldt et Bonpland: $237(*)$

TERRA TYPICA: “...Xalapa, dans la Nouvelle-Espagne...”.

COMENTARIOS TAXONÓMICOS: En la reimpresión de 1911, la descripción está en en la página 160 y la ilustración aparece en la lámina 16 (fig. 7), en lugar de la 11 (fig. 17) (fide Haag-Rutenberg, 1879).

Distribución GEOGRÁficA: Especie endémica de México (Selander, 1983a). Citada en Veracruz.

MÉxICo: Veracruz: Xalapa (Latreille, 1805; Champion, 1892).

Tetraonyx (Tetraonyx) sallei Haag-Rutenberg, 1879

Tetraonyx sallei Haag-Rutenberg, 1879: 299 (*)

TERRA TYPICA: "Cordova (Staat von Veracruz)". Tipo posiblemente en la Colección Sallé del British Museum of Natural History (fide Haag-Rutenberg, 1879).

COMENTARIOS TAXONÓMICOS: Champion (1892: 390) escribe el nombre de esta especie como Tetraonyx sallaei.

DisTRIBUCIÓN GEOGRÁFICA: Especie endémica de México (Selander, 1983a). Citada en Veracruz.

MÉxICo: Veracruz: Córdoba (Haag-Rutenberg, 1879; Champion, 1892; Selander, 1983a).

Tetraonyx (Tetraonyx) sexguttatus (Olivier, 1795)

Mylabris sexguttata Olivier, 1795: nº 47, p. 11 (*)

TERRA TYPICA: "Surinam"

Mylabris atrata Fabricius, 1801: 83 (*)

TERRA TYPICA: "America meridionali".

Lytta (Tetraonyx) sexguttata (Olivier, 1795): Klug, 1825: 449 (*)

Tetraonyx sexguttatus (Olivier, 1795): Blanchard, 1836: 41 (*)

Tetraonyx proteus Haag-Rutenberg, 1879: 260 (*)

TERRA TYPICA: "Mexico, Honduras, Granada".

Tetraonyx proteus var. biguttatus Haag-Rutenberg, 1879: 260 (*)

TERRA TYPICA: "Honduras. Chiriqui". Tipo posiblemente en la Colección Sallé del British Museum of Natural History (fide HaagRutenberg, 1879)

Tetraonyx proteus var. centromacutatus Haag-Rutenberg, 1879: 260 (*) TERRA TYPICA: no indicada.

Tetraonyx proteus var. humboldti Haag-Rutenberg, 1879: $260\left(^{*}\right)$ TERRA TYPICA: "Veracruz". Tipo posiblemente en la Colección Sallé del British Museum of Natural History (fide Haag-Rutenberg, 1879).

Tetraonyx proteus var. sanguinolentus Haag-Rutenberg, 1879: 260 (*) TERRA TYPICA: "Mirador". 
Tetraonyx proteus var. sellatus Haag-Rutenberg, 1879: 260 (*) TERRA TYPICA: "Oaxaca".

Tetraonyx ochraceoguttatus Dugès, 1881: $140(*)$

TERRA TYPICA: "Estado de Veracruz". Vázquez \& Zaragoza (1979) indican como tipo el ejemplar $n^{\circ} 1837$ (IBUNAM 019) de la colección del Instituto de Biología de la Universidad Nacional Autónoma de México.

Tetraonyx sexguttatus var. championi Pic, 1915a: 10 (*) TERRA TYPICA: "Guatemala". El tipo es uno de los ejemplares figurados por Champion (1892: lám. 18).

Tetraonyx sexguttatus var. rufomarginatus Pic, 1915a: 10 (*) TERRA TYPICA: "Guatemala". El tipo es uno de los ejemplares ilustrados por Champion (1892: lám. 18).

Tetraonyx sexguttatus var. championi Borchmann, 1917: 114 (*) TERRA TYPICA: "Guatemala".

Tetraonyx sexguttatus var. haagi Borchmann, 1917: 114 (*) TERRA TYPICA: "Guatemala".

Tetraonyx sexguttatus var. biundulatus Pic, 1930: 21 (*) TERRA TYPICA: "Panama".

COMENTARIOS TAXONÓMICOS: Dugès (1886a: 582) y Champion (1892) consideran a T. ochraceoguttatus Dugès, 1881 en la sinonimia de T. sexguttatus y posteriormente (Dugès, 1889e) en la de T. proteus. HaagRutenberg (1879) señala los nombres no disponibles $T$. ochraceoguttatus Deyrolle, T. humboldti Chevrolat y $T$. biguttatus Chevrolat, para su $T$. proteus.

DisTRIBUCIÓN GEOGRÁFICA: Especie ampliamente distribuida por la región neotropical, incluyendo Argentina, Brasil, Colombia, Guatemala, México, Nicaragua, Panamá y Surinám (Denier, 1935a; Selander, 1983a; Selander \& Martínez, 1984; Selander \& Selander, 1992; Maes \& Huether, 2007). En México ha sido citada en Oaxaca y Veracruz.

MÉxICo: Oaxaca: Oaxaca (Haag-Rutenberg, 1879; Champion, 1892; Selander, 1983a). Veracruz: Xalapa (Champion, 1892; Selander, 1983a); Hacienda El Mirador (Champion, 1892); Veracruz (Dugès, 1889e sub T. proteus; Champion, 1892; Vázquez \& Zaragoza, 1979).

\section{AGRADECIMIENTOS}

Nuestro agradecimiento a Miguel Ángel Alonso Zarazaga (MNCN, Madrid) por sus imprescindibles sugerencias nomenclaturales; a Silvia Santiago y Elisabeth Mejorada (UNAM, México) por las facilidades prestadas para la revisión de la Colección Nacional de Insectos del Instituto de Biología; a Santiago Zaragoza (UNAM, México) por la información sobre la obra inédita de E. Dugès; a Carolina Noreña (MNCN, Madrid) por la traducción de textos en alemán; a Pepe Fernández (MNCN, Madrid) por su ayuda en la localización de referencias y otros imposibles; a Judit Vörös y Otto Merkl (HNHM, Budapest) las facilidades prestadas para el examen de material de la colección Kaszab; a Jose Luis Ruiz (Ceuta) por la revisión de textos y apoyo constante; y finalmente a John D. Pinto (University of California, Riverside) e Ignacio Ribera (MNCN, Madrid) los numerosos comentarios aportados durante la revisión crítica del manuscrito.

Este trabajo ha contado para su realización con los proyectos CGL2004-04680-C10-10/BOS y CGL2007-64621 del Ministerio de Educación y Ciencia, lo que nos ha facilitado el apoyo logístico necesario. La visita a la colección del Magyar
Természettudomány Múzeum (HNHM) en Budapest ha sido financiada por el proyecto Synthesys «European Commission's Research Infrastructure Action».

\section{Referencias}

Adams, C. L. \& Selander, R. B., 1979. The biology of blister beetles of the Vittata group of the genus Epicauta (Coleoptera, Meloidae). Bulletin of the American Museum of Natural History, 162: 139-266.

Agafitei, N. J. \& Selander, R. B., 1980. The first instar larvae of the vittata group of the genus Epicauta (Coleoptera: Meloidae). Journal of the Kansas Entomological Society, 53: 1-26.

AksentJeV, S. I., 1981. [New genera and species of blister beetles (Coleoptera, Meloidae) from Middle Asia.] [en ruso]. Entomologicheskoye Obozreniye, 60: 860-864.

AKSENTJEV, S. I., 1988. [A catalogue of the genus-group taxa of the beetle family Meloidae (Coleoptera).] [en ruso]. Entomologicheskoye Obozreniye, 67: 569-582.

AksentJev, S. I., 1989. Catalogue of the genus group taxa of Meloidae (Coleoptera). Entomological Review, 68(3): 11-26. [Traducción en inglés de Entomologicheskoye Obozreniye, 1988, 67: 569-582.]

ARNOLD, D. C., 1976. Blister beetles of Oklahoma. Oklahoma State University Agricultural Experiment Station, Technical Bulletin, T-145: 1-65.

Ballmer, G., 1979. The puncticollis complex of the genus Epicauta (Coleoptera: Meloidae). The Wasmann Journal of Biology, 37(1-2): 64-88.

BeAuregard, H., 1889. [Notes synonymiques]. Annales de la Societé Entomologique de France, $6^{\text {eme }}$ Série, 9 (Séance du 13 novembre 1889): ccxii-ccxiii.

BeAuregard, H., 1890. Les insectes vésicants. Félix Alcan, París. i-xvi, 1-544, 34 láms.

BERG, C., 1881. Revision der argentinischen Arten der Gattung Cantharis. Entomologische Zeitung herausgegeben von dem entomologischen Vereine zu Stettin, 42: 301-309.

BerG, C., 1883. Doce heterómeros nuevos de la fauna argentina. Anales de la Sociedad Científica Argentina, 15: 66-78.

Betrem, J. B., 1932. Beiträge zur kenntnis des Tribus der Horiini der Familie der Meloiden. I. Die Systematik der Horiini. Treubia, 14(1): 85-102, 1 pl.

Bianchi, F. A., 1962. Notes on the biology of Cissites auriculata (Champion) (Coleoptera: Meloidae). Proceedings of the Hawaiian Entomological Society, 18(1): 111-119.

BlackWelder, R. E., 1945. Checklist of the coleopterous insects of Mexico, Central America, the West Indies, and South America. Part 3. United States National Museum Bulletin, 185: 343-550. 
Blaisdell, F. E., 1918. Synopsis of genus Tegrodera (Coleoptera: Meloidae). The Canadian Entomologist, 50: 333-335.

Blaisdell, F. E., 1925. XIV Expedition to Guadalupe Island, Mexico in 1922. Proceedings of the California Academy of Sciences, Fourth Series, 14: 321-343.

Blanchard, E., 1836. Les Insectes. En: Cuvier, G. Le règne animal distribué d'après son organization, pour servir à l'Histoire Naturelle des animaux et d'introduction à l'Anatomie comparée. Troisième Edition. Tome Troisième. L. Hauman et Cie., Bruxelles.

BlANCHARD, E., 1843. Insectes recueills par Alcide d'Orbigny et décrits par Emile Blanchard et Auguste Brullé. Coléoptères, 2: 200, lám 15. En: D’Orbigny, A. 1843. Voyage dans l'Amérique Méridionale, T.VI.(2éme. Partie) 1837-1843. 222 pp., 32 láms.

Blanchard, E., 1845. Histoire naturelle des Insectes, traitant de leurs moeurs et de leurs métamorphoses en général, et comprenant une nouvelle classification fondée sur leurs rapports naturels. 2. Didot, Paris. 534pp., 20 láms.

Blodgett, S. L., Higgins, R. A. \& Milliken, G. A., 1995. Blister beetle (Coleoptera: Meloidae) mortality evaluated during alfalfa harvest. Journal of Economic Entomology, 88: 398-406.

Bologna, M. A., 1991. Fauna de Italia. XXVIII. Coleoptera Meloidae. Calderini, Bologna. i-xiv, 1-54.

Bologna, M. A. \& Pinto, J. D.,1998. A review of the Afrotropical species of Meloe (Coleoptera, Meloidae) with descriptions of first instar larvae, a key to species and an annotated catalogue. Tropical Zoology, 11: 1959.

Bologna, M. A. \& Pinto, J. D., 2001. Phylogenetic studies of Meloidae (Coleoptera), with emphasis on the evolution of phoresy. Systematic Entomology, 26: 33-72.

Bologna, M. A. \& Pinto, J. D., 2002. The Old World genera of Meloidae (Coleoptera): a key and synopsis. Journal of Natural History, 36: 2013-2102.

Bologna, M. A. \& PINTO, J. D., 2007. Rediscovery of Tetraolytta gerardi (Pic) (Coleoptera: Meloidae), an enigmatic blister beetle from Brazil: redescription and taxonomic placement. Proceedings of the Entomological Society of Washington, 109: 765-772.

Borchmann, F., 1917. Pars 69. Meloidae, Cephaloidae. En: E. Schenkling (ed.). Coleopterorum Catalogus auspiciis et auxilio W. Junk. Junk, Berlin. 208 pp.

Böving, A.G. \& Craighead, F. C., 1930. An illustrated synopsis of the principal larval forms of the order Coleoptera. Entomologica Americana (N.S.), 11(1): 1-351.

BRANDT, J. F. \& ERICHSON, W. F., 1832. Monographia generis Meloes. Nova Acta Physico-Medica Academiae Caesareae Leopoldino-Carolinae Naturae Curiosum, 16 (1)(1831): 101-142, 1 lám.
BrÈthes, J., 1915. Notas entomológicas. Boletín de la Sociedad Physis, 8: 583-584.

BurmeIsTer, H. C. C., 1881. Die argentinischen Canthariden. Entomologische Zeitung herausgegeben von dem entomologischen Vereine zu Stettin, 42: 20-35.

Calvino, M., Ramírez, R. \& Riquelme Inda, J., 1920. El jitomate y sus enfermedades. Boletín de Agricultura (n.s.) de la Secretaría de Agricultura y Fomento (México), 107: 1-72.

CAPinera, J. L., Gardener, D. R. \& Stermitz, F. R., 1985. Cantharidin levels in blister beetles (Coleoptera: Meloidae) associated with alfalfa in Colorado. Journal of Economic Entomology, 78: 1052-1055.

CArrel, J. E. \& EISner, T., 1974. Cantharidin: potent feeding deterrent to insects. Science, 183: 755-757.

Carrel, J. E., McCairel, M. H., Slagle, A. J., Doom, J. P., Brill, J. \& McCormick, J. P., 1993. Cantharidin production in a blister beetle. Experientia, 49: 171-174.

Casey, T. L., 1891. Coleopterological Notices. III. Annals of the New York Academy of Sciences, 4: 9-214.

Champion, G. C., 1891. Families Mordellidae, Rhipidophoridae, Meloidae. In: F. E. Godman \& O. Salvin (eds.). Biologia Centrali-Americana. Coleoptera Heteromera, Vol. 4. pt. 2: 257-368.

Champion, G. C., 1892. Family Meloidae. In: F. E. Godman \& O. Salvin (eds.). Biologia CentraliAmericana. Coleoptera Heteromera, Vol. 4. pt. 2: 369448, láms.: 17-21.

Champion, G. C., 1893. Family Meloidae, supplement to Heteromera. In: F. E. Godman \& O. Salvin (eds.). Biologia Centrali-Americana. Coleoptera Heteromera, Vol. 4. pt. 2: 449-464.

Champion, G. C., 1899. A list of the Cantharidae supplementary to the "Munich" Catalogue. Annales de la Société Entomologique de Belgique, 43: 153-207.

Chevrolat, L. A. A., 1833. Coléoptères du Mexique. 1er. Fascicule. G. Silbermann. Strasbourg. Sin paginar.

Chevrolat, L. A. A., 1834a. Coléoptères du Mexique. $3 e$. Fascicule. G. Silbermann. Strasbourg. Sin paginar.

Chevrolat, L. A. A., 1834b. Coléoptères du Mexique. 4e. Fascicule. G. Silbermann, Strasbourg. 70 pp. Sin paginar.

Chevrolat, L. A. A., 1844. Insectes. In: F. E. GuérinMéneville, Iconographie du Règne animal de G. Cuvier, vol. 3. Bailière, París y Londres: 133-137.

Chevrolat, L. A. A., 1858. Descriptions de Coléoptères de la partie méridionale de l'île de Cuba. Revue et Magasin de Zoologie, 10: 209-211.

Cockerell, T. D. A., 1899. A new meloid beetle parasitic on Anthophora. Psyche, 8: 416-417.

Cockerell, T. D. A., 1900. The name Leonia. Psyche, 9: 11.

Cockerell, T. D. A., 1910. The classification of the Meloidae. Entomological News, 21: 307. 
Cohen, A. C. \& Pinto, J. D., 1977. An evaluation of xeric adaptiveness of several species of blister beetles (Meloidae). Annals of the Entomological Society of America, 70: 741-749.

CINZ. Comisión Internacional de Nomencaltura Zoológica. 2000. Código Internacional de Nomenclatura Zoológica, $4^{a}$ edición. Museo Nacional de Ciencias Naturales - CSIC International Trust for Zoological Nomenclature, Madrid. 156 pp. [Traducción al español de: ICZN. 2000. International Code of Zoological Nomenclature. 4th ed. International Trust for Zoological Nomenclature. London. 306 pp.]

Cros, A., 1928. Revision des genres Horia Fab. et Cissites Latr. (Note rectificative et complementaire). Bulletin de la Société Royale Entomologique d'Égypte, 2-4 (1927): 103-115.

Cros, A., 1937. Description de la larve primarie d'un Meloe du Mexique recueillie par L. Diguet (Meloe tropicus Motsch.). Revue Française d'Entomologie, 4: 192-199.

Cros, A., 1940. Essai de classification des Meloidae algériens. VI Congreso Internacional de Entomología, Madrid, 1935: 312-338.

Crotch, G. R., 1873. Check List of the Coleoptera of America, North of Mexico. Naturalists Agency, Salem, Massachusetts. 136 pp.

DAMPF, A., 1925. Capítulo III. Observaciones acerca de los parásitos encontrados en la langosta. En: C. C. Hoffmann, A. Dampf y G. Varela. Informe de la comisión científica exploradora de la plaga de la langosta en el Estado de Veracruz. Departamento de Salubridad Pública de la República Mexicana. Monografías del Instituto de Higiene, Sección de Parasitología, 3: 85-93.

DE GeER, C., 1775. Mémoires pour servir à l'histoire des insectes. Tome cinquième. P. Hesselberg, Stockholm. 7+448 pp., 16 láms.

Dejean, P. F., 1834. Catalogue des Coléoptères de la collection de M. le Compte Dejean. Edition 2. Livr. 3. Méquignon-Marvis, Paris. Pp.: 117-256.

Dejean, P. F., 1837. Catalogue des Coléoptères de la collection de M. le Compte Dejean. Troisième édition, revue, corrigée et augmentée. 1-4. MéquignonMarvis, Paris. Págs. 1-468.

DenIER, P., 1933. Contribución al estudio de los meloidos americanos. Revista Chilena de Historia Natural, 37: 237-246.

DenIER, P., 1934. Contribución al estudio de los meloidos americanos. II. Ensayo de clasificación de la Pyrota (Dej.) Lec. basada en los caracteres sexuales secundarios de los machos. Revista de la Sociedad Entomológica Argentina, 6: 49-75.

DENIER, P., 1935a. Coleopterorum americanorum familiae meloidarum. Enumeratio synonymica. Revista de la Sociedad Entomológica Argentina, 7: 139-176.
DenIER, P., 1935b. Estudios sobre meloidos americanos. Apuntes críticos de sistemática y de nomenclatura. Revista Argentina de Entomología, 1: 15-28.

Denier, P., 1940. Enumerationi coleopterorum americanorum familiae meloidarum. Corrigenda et addenda. Revista de la Sociedad Entomológica Argentina, 10: 418-425.

DETTNER, K., 1997. Inter- and intraspecific transfer of insect toxic compound cantharidin. In: K. Dettner, G. Bauer \& W. Völkl (eds.). Vertical food web interactions. Springer. Berlin und Heidelberg. Ecological studies, 130: 115-145.

Dillon, L. S., 1950. A new species of Epicauta from Arizona (Col.: Meloidae). Entomological News, 61: 103-104.

Dillon, L. S., 1952. The Meloidae (Coleoptera) of Texas. The American Midland Naturalist, 48: 330420, 3 láms.

Doyen, J. T. \& Opler, P., 1973. Distributional affinities of some xerophilous insects (Coleoptera, Lepidoptera) in central California. Southwestern Naturalist, 18: 305316 .

Dufour, L., 1828. Description d'un genre nouveau d'Insectes de l'ordre des Parasites. Annales des Sciences Naturelles, 13: 62-66, 1 lám.

DuGÈs, E., 1869a. Descripción de algunos meloideos indígenas. La Naturaleza, 1: 100-113. [entrega 4a pág. 100; entrega 5a pág. 105]

DugÈs, E., 1869b. Descripción de algunos meloideos indígenas. La Naturaleza, 1: 125-128. [entrega 6a pág. 125: 1869]

DugÈs, E., 1869c. Descripción de algunos meloideos indígenas. La Naturaleza, 1: 157-160, [entrega 7a pág. 157: 1869]

DuGÈs, E., 1870. Descripción de algunos meloideos indígenas. La Naturaleza, 1: 161-171, láms. 1-2. [entrega 8a pág. 161: 1870]

DuGÈs, E., 1874a. Estudios sobre algunos insectos indígenas. Descripción de una nueva especie de la familia de los Meloideos, Nemognatha zonitoides. La Naturaleza, 3: 47-48.

DUGÈs, E., 1874b. Estudios sobre algunos insectos indígenas. Rectificaciones sinonímicas a la Memoria de los Meloideos publicada en el primer tomo de este periódico, hechas por su autor. La Naturaleza, 3: 48-49.

DugÈs, E., 1877. Descripción de algunos meloideos indígenas. La Naturaleza, 4: 57-67, lám., 2.

DugĖs, E., 1881. Descripción de algunos meloideos indígenas. La Naturaleza, 5: 140-148, lám., 4.

DUGÈs, E., 1886a. Note pour servir à la classification des méloïdes du Mexique. Bulletin de la Societé Zoologique de France, 11: 578-582.

DugÈs, E., 1886b. Addition à la note pour servir à la classification des méloïdes du Mexique. Bulletin de la Societé Zoologique de France, 11: 680. 
DugĖs, E., 1889a. Descripción de la Leonia Rileyi, nuevo género de meloideo, vecino de Hornia (Tomada y traducida del "Insect Life". Vol. 1 nº7. Enero de 1889). Anales del Museo Michoacano, 2: 4-9.

DuGÈs, E., 1889b. Nota segunda sobre la clasificación de los meloideos de la República Mexicana. Anales del Museo Michoacano, 2: 10-15.

DugÈs, E., 1889c. Omisión en la descripción de Leonia Rileyi - Pág. 5. -Línea 21. Anales del Museo Michoacano, 2: 27.

DugÈs, E., 1889d. Sinopsis de los meloideos de la República Mexicana. Anales del Museo Michoacano, 2: $34-40$

DugÈs, E., 1889e. Sinopsis de los meloideos de la República Mexicana. Anales del Museo Michoacano, 2: 49-113 + 107bis-114bis + 194-195.

DuGÈs, E., 1889f. Description of Leonia rileyi, a new meloïd genus near Hornia. Insect Life (U.S. Department of Agriculture), 1(7): 211-213.

ENNS, W. R., 1956. A revision of the genera Nemognatha, Zonitis, and Pseudozonitis (Coleoptera: Meloidae) in America North of Mexico, with a proposed new genus. The University of Kansas Science Bulletin, 37: 685-909.

ENNS, W. R., 1958. Distribution records of two American species of Cissites (Coleoptera-Meloidae). The Coleopterists'Bulletin, 12: 61-64.

ERICHSON, W. F., 1848. Die Insekten. In: R. Schomburg. Reisen in British-Guiana in den Jahren 1840-1844, 3: 533-617.

Fabricius, J. C., 1775. Systema entomologiae. Sistens Insectorum classes, ordines, genera, species etc. Korte, Flesburgi et Lipsiae. i-xxx, 1-832.

FABRICIUS, J. C., 1787. Mantissa Insectorum sistens eorum species nuper detectas adjectis characteribus genericis, differentiis specificis, emendationibus, observationibus. Vol. 1. C.G. Proft. Hafniae. 348 pp.

FABRicius, J. C., 1792. Entomologia Systematica emendata et aucta secundum classes, ordines, genera, species adjectis synonimis, locis, observationibus, descriptionibus. Tom. I, Pars. II. Hafniae. 538 pp.

FABricius, J. C., 1798. Supplementum Entomologiae Systematicae. Proft et Storch. Hafniae, Copenhague. i-ii, $572 \mathrm{pp}$.

FABricius, J. C., 1801. Systema Eleutheratorum secundum ordines, genera, species: adiectis synonymis, locis, observationibus, descriptionibus. Vol. 2. Impensis Bibliopolii Academici Novi. Kiliae. 687 pp.

FALL, H. C., 1901a. List of the Coleoptera of Southern California, with notes on habits and distribution and descriptions of new species. Occasional Papers of the California Academy of Sciences, 8: 1-282.

FAll, H. C., 1901b. Notes on Dichelonycha and Cantharis, with descriptions of new species in other genera. Transactions of the American Entomological Society, 27: 277-310.
FALL, H. C., 1907. Descriptions of new species. In: H. Fall \& T. D. A. Cockerell. The Coleoptera of New Mexico. Transactions of the American Entomological Society, 33: 218-272.

FALL, H. C., 1909. New Coleoptera from the SouthWest. - IV. Canadian Entomologist, 41: 161-170.

Fischer, J. B., 1827. Tentamen conspectus Cantharidiarum. Monachii. $26 \mathrm{pp}$.

FISCHER DE WALDHEIM, G., 1824: Entomographia Imperii Rossici; Genera Insectorum systematice exposita et analysi iconographica instructa, vol. 2. 1823. Typis Augusto Semen. Mosquae. xx + 264 pp., 40 láms.

FrIVALDSZKY, J., 1877. Adnotationes coleopterologicae. Termeszetrajzi Füzetek, 1: 136.

Forster, J. R., 1771. Novae species insectorum. Centuria I. London. $100 \mathrm{pp}$.

GAHAN, C. J., 1908. Notes on the coleopterous genera Horia Fab., and Cissites Latr., and a list of the described species. Annals and Magazine of Natural History (Series 8), 2: 199-204.

GANGLBAUER, L., 1907. Gegestandt des vortrages bildet die Metamorphose und Systematik der Meloiden. Verhandlungen der kaiserlich-königlichen zoologisch-botanischen Gessellschaft in Wien, 57: 101106.

García-París, M., Parra-Olea, G. \& Settanni, C., 2005. Primer registro de Epicauta segmenta (Say, 1824) (Coleoptera: Meloidae) en Querétaro (México). Graellsia, 61(2): 257-258.

GeBler, F., 1817. Insecta Sibiriae rariora, descripsit. Decas prima. Mémoires de la Société Impériale des Naturalistes de Moscou, 5: 315-333.

Gemminger, M., 1870. Geänderte Namen. Nachstehende Art- oder Gattungsnamen ändere ich, wie folgt, ab. Coleopterologische Hefte, 6: 119-124.

Gemminger, M. \& Harold, B., 1870. Catalogus Coleopterorum hucusque descriptorum synonymicus et systematicus. Gummi, Monachi. 7: 1801-2180.

Genaro, J. A., 1996. Resumen del conocimiento sobre los meloidos de Cuba (Insecta: Coleoptera). Caribbean Journal of Science, 32: 382-386.

Geofrroy, E. L., 1762. Histoire abrégée des Insectes qui se trouvent aux environs de Paris, dans laquelle ces animaux sont rangés suivant un ordre méthodique. Nouvelle Édition, revue, corrigée, \& augmentée d'un Supplément considérable. Vol 1 [1764?]. Durand, Paris. 1-28, 1-523, 10 lám.

GERMAR, E. F., 1824. Insectorum species novae aut minus cognitae, descriptionibus illustratae. J.C. Hendelii et Filii. Halae. 624 pp.

GiBSon, A., 1912. Blister beetles. Entomological Society of Ontario, Forty-Second Annual Report, 1911, 36: 83-88.

Gmelin, J. F., 1789. Pp. 2016-2020 in Linnaeus, C. 1789. Systema Naturae per Regna tria Naturae, secundum 
Classes, Ordines, Genera, Species, cum characteribus, differentiis, synonymis, locis. Ed. XIII, aucta, reformata. Tomus 1, Pars 4. Salvii, Holmiae.

Gowdey, C. C., 1926. Catalogus insectorum jamaicensis. Department of Agriculture of Jamaica, Entomological Bulletin, 46(1,2): 1-114.

GuptA, A. P., 1965. The digestive and reproductive systems of the Meloidae (Coleoptera) and their significance in the classification of the family. Annals of the Entomological Society of America, 58: 442-474.

Gyllenhal, L., 1810. Insecta Suecica descripta, Classis I, Coleoptera sive Eleutherata, tomus 1, pars 2. F.J. Leverentz. Scaris. 660 pp.

HaAG-RutenBerg, J. G., 1879. Beiträge zur Kenntniss der Canthariden. I. Tetraonyx. Entomologische Zeitung herausgegeben von dem entomologischen Vereine zu Stettin, 40: 249-275, 287-314.

HaAg-Rutenberg, J. G., 1880. Beiträge zur Kentniss der Canthariden. Deutsche Entomologische Zeitschrift, 24: $17-90$

Haldeman, S. S., 1852. Appendix C. Insects. In: H. Stansbury (ed.) Exploration and survey of the valley of the Great Salt Lake of Utah including a reconaissance of a new route through the Rocky Mountains. Washington, DC: 366-378.

Halstead, J. A. \& Haines, R. D., 1992. New distributional records for some candidate species of Lytta in California. The Pan-Pacific Entomologist, 68: 68-69.

HaVELKA, P., 1980. Eine interessante Ölkäferart (Meloe violaceus) (Coleoptera, Meloinae) an einem Trockenstandort im Rheinvorland bei Eggenstein. Pfälzer Heimat, 31: 110-112.

HernÁNDEZ, J., 1960. Estudio taxonómico y datos biológicos de la familia Meloidae (Coleoptera) en los alrededores de Monterrey, N. L. Tesis, Escuela de Agricultura, Instituto Tecnológico y de Estudios Superiores de Monterrey. $163 \mathrm{pp}$.

Herrera, A. \& MendozA, G., 1866. Apuntes para la Monografía de los insectos vexicantes indígenas. Gaceta Médica de México, 2(17): 264-266.

Herrera, A. \& MendozA, G., 1867. Apuntes para la monografía de los insectos vexicantes indígenas. Gaceta Médica de México, 3(1): 13-15.

HEYDEN, L. vON, 1890. Ueber Epicauta bicolor Fischer, armeniaca Fald. und dichroa Leconte. Wiener Entomologische Zeitung, 9(3): 99.

HorN, G. H., 1868. New species of Coleoptera from the Pacific district of the United States. Transactions of the American Entomological Society, 2: 129-140.

Horn, G. H., 1870. Contributions to the coleopterology of the United States. Transactions of the American Entomological Society, 3: 69-97.

HoRn, G. H., 1873. Revision of the species of several genera of Meloidae of the United States. Proceedings of the American Philosophical Society, 13(90): 88-117.
Horn, G. H., 1874. Descriptions of new species of United States Coleoptera. Transactions of the American Entomological Society, 5: 20-43.

HoRn, G. H., 1875. Synonymical notes and description of new species of North American Coleoptera. Transactions of the American Entomological Society, 5: 126-156.

Horn, G. H., 1878. Contributions to the coleopterology of the United States, No. 2. Transactions of the American Entomological Society, 7: 51-60.

Horn, G. H., 1883. Miscellaneous notes and short studies of North American Coleoptera. Transactions of the American Entomological Society, 10: 269-312.

Horn, G. H., 1885. Studies among the Meloidae. Transactions of the American Entomological Society, 12: 107116 , lám. 5

HorN, G. H., 1891. New species and miscellaneous notes. Transactions of the American Entomological Society, 18: 32-47, tab. 1.

Horn, G. H., 1894. The Coleoptera of Baja California. Proceedings of the California Academy of Sciences, 4: 302-449, lám. 7-8.

Horn, G. H., 1895. Coleoptera of Baja California. Supplement I. Proceedings of the California Academy of Sciences, Second Series, 5 (1893-94): 225-259.

HorN, G. H., 1896. Coleoptera of Baja California. Supplement II. Proceedings of the California Academy of Sciences, 6: 367-381.

Horsfall, W. R., 1943. Biology and control of common blister beetles in Arkansas. University of Arkansas Agricultural Experiment Station Bulletin, 436: 1-55.

IABloKoff-Khnzorian, S. M., 1983. Fauna Armianskoi SSR (Meloidae, Alleculidae). Akademia Nauk Armianskoi SSR, Institut Zoologii, Erevan. 156 pp. [en ruso].

Illiger, J. C. W., 1804. Bemerkungen zum zweiten Theile von Fabricius Systema Eleutheratorum. Magazin für Insektenkunde, 3: 160-180.

ILLIGER, J. C. W., 1807. Vorschlang zur Aufnahme in Fabricischen Systeme fehlender Käfergattungen. Magazin für Insektenkunde, 6: 318-349.

JimÉNEZ, L. M., 1866a. Dictámen de la Comisión de Ciencias auxiliares sobre los insectos presentados á la Sociedad por el Sr. Barranco. Gaceta Médica de México, 2(15): 227-230.

JiMÉNEZ, L. M., 1866b. Especie nueva del género Cantharis (Cantharis fasciolata.). Gaceta Médica de México, 2(16): 253-256.

KASZAB, Z., 1958. Neue Meloiden-Namen (Coleoptera). Annales Historico-Naturales Musei Nationalis Hungarici, Pars Zoologica, 50: 189-191.

KasZAB, Z., 1959. Phylogenetische Beziehungen des Flügelgeäders der Meloiden (Coleoptera), nebst Beschreibung neuer Gattungen und Arten. Acta 
Zoologica Academiae Scientiarum Hungaricae, 5: 67-114.

KaszaB, Z., 1960. Einige neue Meloiden (Coleoptera) des Museums G. Frey. Entomologische Arbeiten aus dem Museum Georg Frey, 11: 283-290.

KASZAB, Z., 1963a. Studien über Meloiden (Coleoptera). Annales Historico-Naturales Musei Nationalis Hungarici, Pars Zoologica, 55: 335-346.

KASZAB, Z., 1963b. Merkmale der Adaptation, Spezialisation, Konvergenz, Korrelation und Progression bei den Meloiden (Coleoptera). Acta Zoologica Academiae Scientiarum Hungaricae, 9: 137-175.

KaszaB, Z., 1969. The system of the Meloidae (Coleoptera). Memorie della Società Entomologica Italiana, 48: 241-248.

KIRBY, W., 1802. Monographia apum Angliae; or an attempt to divide into their Natural Genera and Families, Such Species of the Linnean Genus Apis as have been discovered in England: with descriptions and observations. To which are prefixed some introductory remarks upon the Class Hymenoptera, and a Synoptical Table of the Nomenclature of the external parts of these Insects. Volume 2. J. Raw. Ipswich. 388 pp.

KIRBY, W., 1818. A century of insects including several new genera described from his cabinet. Transactions of the Linnean Society of London, 12: 375-435, 2 láms.

KIRBY, W., 1837. Insects. In: J. Richardson. Fauna Boreali-Americana, or The Zoology of the Northern parts of British America containing descriptions of the objects of Natural History collected on the late northern land expeditions, under command of Captain Sir John Franklin, R.N. Vol. 4. 325 pp., 8 láms. J. Fletcher, Norwich.

KLUG, J. C. F., 1825. Entomologiae Brasilianae specimen alterum, sistens insectorum coleopterorum nondum descriptorum centuriam. Nova Acta Physico-Medica Academiae Caesareae-Leopoldino-Carolinae. Naturae Curiosorum, 12 (2): 421-476, láms. XL-XLIV.

Knowlton G. F. \& TAYlor, G. P., 1952. Beetles. Coleoptera. Records and notes, largely from Utah. Utah Agricultural Experiment Station. Mimeo Series, 389.

LACORDAIRE, J. T., 1859. Histoire naturelle des Insectes. Genera des Coléoptères ou exposé méthodique et critique de tous les genres proposés jusqu'ici dans cet ordre d'Insectes. Tome cinquième. Atlas. Roret. Paris. 750 pp.

Laporte de CAstelnau, M. L. F., 1840. Histoire Naturelle des Insectes Coléoptères. Vol. 2. In: Histoire Naturelle des Animaux Articulés. Annelides, Crustacés, Arachnides, Myriapodes et Insectes. P. Duménil. Paris. 564 pp., 38 láms.

Latreille, P. A., 1802. Histoire naturelle, générale et particulière des Crustacés et des Insectes. Ouvrage faisant suite aux oeuvres de Leclerc et de Buffon, et partie du cours complet d'Histoire naturelle rédigé par C.S. Sonini. Tomo 3. Dufart. Paris. i-xii +467 pp.

Latreille, P. A., 1804a. En: Tableau méthodique des insectes. In: Tableaux méthodiques d'Histoire naturelle. In: Nouveau dictionnaire d'Histoire naturelle. Déterville. Paris. Tomo 24.

Latreille, P. A., 1804b. Histoire naturelle, générale et particulière des Crustacés et des Insectes. Ouvrage faisant suite à l'Histoire naturelle générale et particulière par Leclerc et de Buffon et partie du cours complet d'Histoire naturelle rédigé par C.S. Sonini. Dufart. Paris. Tomo 10: i-xii + 415 pp.

Latreille, P. A., 1805. Insectes de l'Amérique équinoxiale recuellis pendant le voyage de MM. de Humboldt et Bonpland, et décrits par M. Latreille. In: A. von Humboldt \& A. Bonpland. Voyage de Humboldt et Bonpland aux regions équinoxiales $d u$ Nouveau Continent..., Part. II Zoologie, vol. 1, $\mathrm{n}^{\circ}$ 9: 127-252, láms.: 15-24. Paris. Reimpreso en 1811 con el mismo título. In: A. von Humboldt \& A. Bonpland. Recueil d'observations de zoologie et d'anatomie comparée, faites dans l'Océan Atlantique, dans l'intérieur du Nouveau Continent dans la Mer du Sud, Vol. 1, Parte 1: 127-259, láms.: 15-24. Schoell. Paris.

LAtreille, P. A., 1810. Considérations générales sur l'ordre naturel des animaux composant les classes des Crustacés, des Arachnides et des Insectes; avec un tableau méthodique de leurs genres disposés en familles. Schoell. Paris. 444 pp.

Le Peletier, A. L. M. \& Audinet-Serville, J. G., 1825. Sitaris [pp. 438-440], Tetraonyx [pp. 595-596], Zonite [pp. 820-821], and Gnathie [pág. 821]. In: P. A. Latreille. Encyclopédie méthodique. Histoire naturelle. Entomologie, ou Histoire naturelle des Crustacés, des Arachnides et des Insectes. vol 10. V. Agasse, Paris.

LEACH, W. E., 1815. Further observations on the genus Meloë, with descrition of six exotic species. Transactions of the Linnean Society of London, 11(1810): 242-251, lám. 18.

LeConte, J. L., 1851. Descriptions of new species of Coleoptera, from California. Annals of the Lyceum of Natural History of New York, 5: 125-216.

LeConte, J. L., 1853. Synopsis of the Meloidae of the United States. Proceedings of the Academy of Natural Sciences of Philadelphia, 6: 328-350.

LeConTe, J. L., 1854a. Descriptions of some new Coleoptera from Texas, chiefly collected by the Mexican Boundary Commission. Proceedings of the Academy of Natural Sciences of Philadelphia, 6 (1853): 429-448.

LeConte, J. L., 1854b. Descriptions of some new Coleoptera from Oregon, collected by Dr. J.G. Cooper of the North Pacific R.R. Expedition, under Gov. J.J. Stevens. Proceedings of the Academy of Natural Sciences of Philadelphia, 7: 16-20. 
LeConte, J. L., 1854c. Notice of some Coleopterous Insects, from the collection of the Mexican Boundary Commission. Proceedings of the Academy of Natural Sciences of Philadelphia, 7: 79-85.

LeConte, J. L., 1854d. Description of new Coleoptera collected by Thos. H. Webb, M.D., in the years 1850 51 and 52, while Secretary of the U.S. and Mexican Boundary Commission. Proceedings of the Academy of Natural Sciences of Philadelphia, 7: 220-225.

LeCONTE, J. L., 1857. Report upon the insects collected on the survey. In: Reports of explorations and surveys to ascertain the most practicable and economical route for a railroad from the Mississippi River to the Pacific Ocean made under the direction of the Secretary of War, in 1853-5, vol. 12, book 2, part 3. Láms.: 1-2, pp.: 1-52. Washington.

LeConte, J. L., 1858a. Catalogue of Coleoptera of the regions adjacent to the boundary line between the United States and Mexico. Journal of the Academy of Natural Sciences of Philadelphia, ser. 2, 4: 9-42.

LeConTE, J. L., 1858b. Descriptions of new species of Coleoptera, chiefly collected by the United States and Mexican Boundary Commission, under Major W. H. Emory, U.S.A. Proceedings of the Academy of Natural Sciences of Philadelphia, 10: 58-89.

LeConte, J. L., 1859. Descriptions of some genera and species of Coleoptera from the vicinity of the southern boundary of the United States of America. Arcana Naturae (Archives d'Histoire Naturelle), 3: 121-128, 2 láms.

LeConte, J. L., 1861. New species of Coleoptera inhabiting the Pacific district of the United States. Proceedings of the Academy of Natural Sciences of Philadelphia, 13: 338-359.

LeConte, J. L., 1862. Classification of the Coleoptera of North America. Prepared for the Smithsonian Institution. Part I. Smithsonian Miscellaneous Collections, 136: i-xxv, 1-286.

LeConte, J. L., 1866. New species of North American Coleoptera. Prepared for the Smithsonian Institution. Smithsonian Miscellaneous Collections, 167: 87-177.

LeConte, J. L., 1868. New Coleoptera collected on the survey for the extension of the Union Pacific Railway, E. D. from Kansas to Fort Craig, New Mexico. Transactions of the American Entomological Society, 2: 49-64.

LeConte, J. L., 1870. Synonymical notes on NorthAmerican Coleoptera. Annals and Magazine of Natural History, 6: 394-404.

LeConte, J. L., 1883. [Comentarios intercalados en el texto original de Say]. In: The complete writings of Thomas Say on the entomology of North America, vol. 2, Boston.

Leng, C. W., 1920. Catalogue of the Coleoptera of America, North of Mexico. Meloidae. J.D. Sherman, Jr. Mount Vernon: 157-160, 354.
Leng, C. W. \& Mutchler, A. J., 1914. A preliminary list of the Coleoptera of the West Indies as recorded to Jan. 1, 1914. Bulletin of the American Museum of Natural History, 33: 391-493.

LEWIS, E., 2004. A United States record for the genus Cissites Latreille (Coleoptera: Meloidae: Zonitini). The Coleopterists' Bulletin, 58(4): 635-636.

Linnaeus, C., 1758. Systema Naturae per Regna tria Naturae, secundum Classes, Ordines, Genera, Species, cum characteribus, differentiis, synonymis, locis. Ed. X. Vol. 1. Salvii. Holmiae. 824 pp.

LinNAEUS, C., 1763. Amoenitates academicae; seu dissertationes variae physicae, medicae, botanicae, antehac seorsim editae, nunc collectae et auctae, cum tabulis aeneis. Volumen sextum. L. Salvii. Holmiae. $3+486$ pp., 5 láms.

Linnaeus, C., 1767. Systema Naturae per Regna tria Naturae, secundum Classes, Ordines, Genera, Species, cum characteribus, differentiis, synonymis, locis. Editio Duodecima, reformata. Tomus I, Pars II. Salvii, Holmiae.

LINSLEY, E. G., 1942. Systematics of the meloid genera Hornia and Allendesalazaria (Coleoptera). University of California Publications in Entomology, 7: 169-188.

Linsley, E. G., MacSwain, J. W. \& Michener, C. D., 1980. Nesting biology and associates of Melitoma (Hymenoptera, Anthophoridae). University of California Publications in Entomology, 90: 1-45.

Linsley, E. G., MacSwain, J. W. \& SMith, R. F., 1956. Biological observations on Ptilothrix sumichrasti (Cresson) and some related groups of emphorine bees (Hymenoptera, Anthophoridae). Bulletin of the Southern California Academy of Sciences, 55: 83-101.

LuCAS, P. H., 1859. Entomologie. In: F. Laporte de Castelnau. Animaux nouveaux ou rares recueillis pendant l'expédition dans les parties centrales de l'Amérique du Sud, de Rio de Janeiro a Lima, et de Lima au Para; exécutée par ordre du Gouvernement français pendant les années 1843 a 1847, sous la direction du Compte Francis de Castelnau. Septième Partie. Zoologie. 1855. P. Bertrand. Paris. 204 pp., 18 láms.

MacSwain, J. W., 1951a. A new genus of Meloidae from North America. The Pan-Pacific Entomologist, 27: 58.

MacSwaIn, J. W., 1951b. New North American species of Nemognatha and Zonitis. The Pan-Pacific Entomologist, 27: 72-80.

MaCSwaIn, J. W., 1952. A synopsis of the genus Gnathium, with description of new species (Coloeptera, Meloidae). The Wasmann Journal of Biology, 10: 205-224.

MacSWAin, J. W., 1956. A classification of the first instar larvae of the Meloidae (Coleoptera). University of California Publications in Entomology, 12: 1-182.

MacSwain, J. W., 1958. Taxonomic and biological observations on the genus Hornia (Coleoptera: 
Meloidae). Annals of the Entomological Society of America, 51: 390-396.

MAEs, J. M., 1989. Catálogo de los insectos controladores biológicos en Nicaragua. VII. Insectos depredadores del orden Coleoptera. Super Familia Meloidea. Revista Nicaragüense de Entomología, 9: 111-120.

Maes, J. M. \& Huether, J. P., 2007. Catálogo ilustrado de los Meloidae (Coleoptera) de Nicaragua y otras especies contenidas en el Museo Entomológico de León. Revista Nicaragüense de Entomología, 67(Suplemento 3): $1-90$.

MANNERHEIM, C. G., 1843. Beitrag zur Kaefer-Fauna der Aleutischen Inseln, der Insel Sitkha und NeuCaliforniens. Bulletin de la Société Impériale des Naturalistes de Moscou, 16: 175-238.

ManNerheim, C. G., 1852. Zweiter Nachtrag zur Kaefer-Fauna der Nord-Amerikanischen Laender des Russischen Reiches. Bulletin de la Société Impériale des Naturalistes de Moscou, 25(1): 283-387.

MartíneZ, A., 1992. Los Meloidae de Salta, Argentina (Coleoptera). Insecta Mundi, 6: 1-12.

Mathieu, J. M., 1983. Two new Epicauta from Mexico (Coleoptera: Meloidae). Proceedings of the Entomological Society of Washington, 85: 156-160.

Maydell, G. G., 1934. New species of North American Meloidae (Coleoptera). Transactions of the American Entomological Society, 60: 327-336.

MAYDELL, G. G., 1935. A new species of blister beetle form Arizona. Journal of the Washington Academy of Sciencies, 25(2): 72.

Melsheimer, F. F., 1846. Descriptions of new species of Coleoptera of the United States. Proceedings of the Academy of Natural Sciences of Philadelphia, 3: 53-66.

MendozA, G. \& Herrera, A., 1867. Cantharis erytrotora. Pág. 14. In: A. Herrera y G. Mendoza. Apuntes para la monografía de los insectos vesicantes indígenas (continúa). Gaceta Médica de México, 3(1): 13-15.

Mickel, C. E., 1928. A new species of meloid beetle, with a key to the North American species of the genus Leonidia Cockerell (Coleop.). Entomological News, 39: 38-41.

Mickel, C. E., 1929. The North American sitarine blister beetles (Coleop.: Meloidae). Entomological News, 40: $1-7$.

Motschulsky, V., 1856. Voyages. Lettres de M. de Motschulsky à M. Ménétriés. No. 4. St. Petersbourg le 25 Oct. 1855. Études Entomologiques, Helsingfors, 5: 21-38.

MotschUlsky, V., 1872. Enumération des nouvelles espèces de Coléoptères rapportées de ses voyages. Bulletin de la Société Impériale des Naturalistes de Moscou, 45(3): 23-55.

Mulsant, E., 1857. Histoire naturelle des Coléoptères de France. Vésicants. Magnin, Blanchard \& Cie., Paris. 201 pp., 1 lám.
Mulsant, E. \& Rey, C., 1858. Cup-d'oeil sur les insectes de la famille des Cantharidiens accompagné de la description de diverses espéces nouvelles ou peu connues. Opuscules Entomologiques, Huitième Cahier: 45-192, 1 lám. Paris.

Nash, D., 1976. Some interesting Coleoptera from Northeast Essex, England. The Entomologist's Record and Journal of Variation, 88: 39-43.

Olivier, A. G., 1789. Encyclopédie méthodique ou par ordre de matières précédée d'une Vocabulaire universal servant de Table pour tout l'Ouvrage. Dictionnaire des Insectes. 4. Panckoucke, Paris. 1-44, 45-331.

Olivier, A. G., 1790. Encyclopédie méthodique ou par ordre de matières précédée d'une Vocabulaire universal servant de Table pour tout l'Ouvrage. Dictionnaire des Insectes. 5. Panckoucke. Paris.

Olivier, A. G., 1795. Entomologie, ou Histoire Naturelle des Insectes, avec leurs caractères génériques et spécifiques, leur description, leur synonymie et leur figure enluminée. 46. Coléoptères. Tome Troisième. Lanneau. Paris. 557 pp.

Pallas, P. S., 1776. Reise durch verschiedene Provinzen des Rußischen Reichs. Dritter Theil vom Jahr 1772, und 1773. [Reise im ostlichen Sibirien und bis in Dauurien. 1772stes Jahr. Dritter Theil.]. $20+454$ pp., 2 mapas. [Reise aus Sibirien zurück bis an die Wolga im 1773sten Jahr. Des dritten Theils zweytes Buch.]. 455-760 pp., $26+8$ láms. Kayserliche Academie der Wißenschaften, Saint Petersburg.

PAllas, P. S., 1798. Icones insectorum praesertium Rossiae Sibiriaecque peculiarium, quae collegit et descriptionibus illustravit. Fasciculus tertius. Sumtu Wolgangi Waltheri, Erlangae. Pp.: 97-104, 2 láms.

PAOLI, G., 1937. Nota sulla biologia e sulla filogenesi dei Meloidi. Memorie della Società Entomologica Italiana, 16: 71-96.

PAPP, C. S., 1960. Distributional records on some North American beetles with remarks on their ecology in southern California. (Notes on North American Coleoptera, No. 9). Entomological News, 71: 69-77.

PARKer, F. D., 1977. Biological notes on some Mexican bees (Hymenoptera: Megachilidae, Anthophoridae). The Pan-Pacific Entomologist, 53: 189-192.

PARKer, J. R. \& WAKeland, C., 1957. Grasshopper eggs pods destroyed by larvae of bee flies, blister beetles, and ground beetles. United States Department of Agriculture Technical Bulletin, 1165. 29 pp.

PASCOE, F. P., 1862. Notices of new or little-known genera and species of Coleoptera. Part I. The Journal of Entomology, 1: 36-64, lám. III.

Peñafiel Barranco, A., 1866. Estudio sobre dos especies de cantáridas mexicanas. Gaceta Médica de México, 2(15): 225-227, láms. 1-3.

Perty, J. A. M., 1830. De insectorum in America meridionali habitantium vitae genere, moribus ac distri- 
butione geographica observationes nonnullae. 44 pp. En: Delectus animalium articulatorum, quae in itinere per Brasiliam annis MDCCCXVIIMDCCCXX collegerunt J.B. de Spix et C.F.Ph. de Martius; digesit, descripsit, pingenda curavit Maximilianus Perty, jussu et auspiciis Maximiliani Josephi I. Monachii. iii $+44+224$ pp.

PIC, M., 1910. Coléoptères exotiques nouveaux ou peu connus (suite). L'Échange, 26(301): 5-7.

PIC, M., 1914. Nouveau genre, espèces et variétés nouvelles de diverses familles. Mélanges ExoticoEntomologiques, 11: 2-20.

PIC, M., 1915a. Nouvelles espèces de diverses familles. Mélanges Exotico-Entomologiques, 15: 2-24.

PIC, M., 1915b. Nouvelles espèces de diverses familles. Mélanges Exotico-Entomologiques, 16: 14-24.

PIC, M., 1916. Descriptions abregées diverses. Mélanges Exotico-Entomologiques, 21: 2-20.

PIC, M., 1919. Coléoptères exotiques en partie nouveaux (suite). L'Échange, 35(391): 1-3.

PIC, M., 1924. Nouveautés diverses. Mélanges ExoticoEntomologiques, 42: 1-32.

PIC, M., 1927. Coléoptères du Globe. Mélanges ExoticoEntomologiques, 50: 1-36.

PIC, M., 1929. De l'utilité des noms pour les varietés. Bulletin de la Société Entomologique de France, 1929(4): 80-82.

PIC, M., 1930. Nouveautés diverses. Mélanges ExoticoEntomologiques, 56: 1-36.

PINTO, J. D., 1972a. A synopsis of the bionomics of Phodaga alticeps (Coleoptera: Meloidae) with special reference to sexual behavior. The Canadian Entomologist, 104: 577-595.

PINTO, J. D., 1972b. Comparative courtship behavior of Negalius, Phodaga and Cordylospasta, three closely related genera of blister beetles (Coleoptera: Meloidae). Journal of the Kansas Entomological Society, 45: 459-476.

PinTo, J. D., 1974a. Courtship behavior in Linsleya compressicornis and its taxonomic significance (Coleoptera: Meloidae). The Pan-Pacific Entomologist, 50: 1-8.

PINTO, J. D., 1974b. The occurrence of Spastonyx macswaini in California, and a description of its first instar larva (Coleoptera: Meloidae). The Pan-Pacific Entomologist, 50: 418-422.

PinTo, J. D., 1975a. A taxonomic study of the genus Tegrodera (Coleoptera: Meloidae) with special reference to sexual behavior. The Canadian Entomologist, 107: 45-66.

PINTO, J. D., 1975b. The taxonomy of three species of maculate Epicauta (Coleoptera: Meloidae), with descriptions of courtship behavior and first instar larvae. Journal of the Kansas Entomological Society, 48(4): 429-452.
PinTo, J. D., 1975c. Intra- and interspecific courtship behavior in blister beetles of the genus Tegrodera (Meloidae). Annals of the Entomological Society of America, 68: 275-285.

PINTO, J. D., 1977. Comparative sexual behavior in blister beetles of the subtribe Eupomphina (Coleoptera: Meloidae), and an evaluation of its taxonomic significance. Annals of the Entomological Society of America, 70: 937-951.

Pinto, J. D., 1979. A classification of the genus Eupompha (Coleoptera: Meloidae). Transactions of the American Entomological Society, 105: 391-459.

PINTO, J. D., 1980. Behavior and taxonomy of the Epicauta maculata group (Coleoptera: Meloidae). University of California Publications in Entomology, 89: 1-111.

PInTO, J. D., 1982. New synonymies, lectotype designations, and other notes on North American Epicauta (Coleoptera: Meloidae). Proceedings of the Entomological Society of Washington, 84(2): 402408.

Pinto, J. D., 1983. A new Eupompha from Baja California with additional information on E. decolorata (Horn) (Coleoptera: Meloidae). The Pan-Pacific Entomologist, 58 (1982): 184-195.

Pinto, J. D., 1984a. A taxonomic review of Cysteodemus LeConte, Phodaga LeConte and Pleuropasta Wellman (Coleoptera: Meloidae: Eupomphina) with a new generic synonymy. Proceedings of the Entomological Society of Washington, 86: 127-143.

PINTO, J. D., 1984b. New generic synonymies in the Epicautina (Coleoptera: Meloidae: Meloinae). Proceedings of the Entomological Society of Washington, 86: 378-382.

PINTO, J. D., 1984c. Cladistic and phenetic estimates of relationship among genera of eupomphine beetles (Coleoptera: Meloidae). Systematic Entomology, 9: 165-182.

PINTO, J. D., 1985. A new species of Lytta (Coleoptera: Meloidae) from Baja California with notes on relationships in the subgenus Paralytta. The Coleopterists' Bulletin, 39: 335-339.

PinTo, J. D., 1991. The taxonomy of North American Epicauta (Coleoptera: Meloidae), with a revision of the nominate subgenus and a survey of courtship behavior. University of California Publications in Entomology, 110: i-x, 1-372, 40 láms.

Pinto, J. D., 2001. Two new species of Zonitis F. (Coleoptera: Meloidae) from Southwestern North America, with comments on generic definitions in the Nemognathinae. Proceedings of the Entomological Society of Washington, 103(2): 319-324.

Pinto, J. D. \& Bologna, M. A., 1997. Lyttana priapica, a new genus and species of Meloidae (Coleoptera) from South America. The Coleopterists'Bulletin, 51: 371-377. 
Pinto, J. D. \& Bologna, M. A., 1999. The New World genera of Meloidae (Coleoptera): a key and synopsis. Journal of Natural History, 33: 569-620.

Pinto, J. D. \& Selander, R. B., 1970. The bionomics of blister beetles of the genus Meloe and a classification of the New World species. Illinois Biological Monographs, 42: 1-222.

Pliginsky, V., 1914. Les Meloines (Coleoptera, Meloidae) de la collection de V. Motschulsky. Revue russe d'entomologie / Russkoe entomologiceskoe obozrenie, 14: 254-261. [en ruso].

Poole, R. W. \& Gentili, P. (eds.). 1996. Nomina Insecta Nearctica. A Check List of the Insects of North America. Vol. 1: Coleoptera, Strepsistera. Entomological Information Services, Rockville, Maryland.

Quintero M., M.T. \& Canales, I., 1987. Un caso de asociación parasitaria de un coleóptero Meloidae con Apis mellifera. Veterinaria México, 18: 135-138.

RADFORD, K. W., 1960. The blister beetles of Baja California and adjacent islands (Coleoptera: Meloidae). M.S. Thesis, University of Arizona.

RILEY, C. V., 1877a. On the larval characters and habits of the blister beetles belonging to the genera Macrobasis LeC. and Epicauta Fabr., with remarks on other species of the family Meloidae. Transactions of the Academy of Science of Saint Louis, 3: 544-562, lám. 5.

RiLey, C. V., 1877b. On a new remarkable new genus in the Meloidae infesting mason-bee cells in the United States. Transactions of the Academy of Sciences of Saint Louis, 3: 563-565, lám. 5.

SÁnchez Soto, S., 2001. Ocurrencia de Lamprosema indicata (Fabricius) (Lepidoptera: Pyralidae) en el cultivo de frijol en Tabasco, México. Folia Entomológica Mexicana, 40(2): 281-282.

SAY, T., 1817. Descriptions of several new species of insects. Journal of the Academy of Natural Sciences of Philadelphia, series 1, 1: 19-23. [Obra consultada en la reedición recopilatoria con comentarios de LeConte, 1883].

SAY, T., 1824a. Descriptions of the coleopterous insects collected in the late expedition to the Rocky Mountains, performed by order of Mr. Calhoun, Secretary of War, under the command of Major Long. Journal of the Academy of Natural Sciences of Philadelphia, 3: 238-282. [Obra consultada en la reedición recopilatoria con comentarios de LeConte, 1883].

SAY, T., 1824b. Descriptions of the coleopterous insects collected in the late expedition to the Rocky Mountains, performed by order of Mr. Calhoun, Secretary of War, under the command of Major Long. Journal of the Academy of Natural Sciences of Philadelphia, 3[1823]: 298-331. [Obra consultada en la reedición recopilatoria con comentarios de LeConte, 1883].
SAYlOR, L. W., 1940. Two new generic names for South American Coleoptera. Proceedings of the Entomological Society of Washington, 42: 46-47.

Schoeb, T. R. \& Panciera, R. J., 1978. Blister beetle poisoning in horses. Journal of the American Veterinary Medical Association, 173(1): 75-77.

SCHOENHERR, C. J., 1817. Synonymia Insectorum, oder: Versuch einer Synonymia aller bisher bekannten Insecten; nach Fabricii Systema Eleutheratorum. Volumen 1(3). Lewerentzischen Buchdruekerey. Skara. xi +506 pp.

SCHRANK, F. P., 1781. Enumeratio insectorum austriae indigenorum. Klett et Frank, Augustae Vindelicorum. 548 pp.

SElANDER, R. B., 1954a. Notes on the tribe Calospastini, with description of a new subgenus and species of Calospasta (Meloidae). The Coleopterists'Bulletin, 8: 11-18.

Selander, R. B., 1954b. Notes on Mexican Meloidae (Coleoptera). Journal of the Kansas Entomological Society, 27: 84-97.

SElander, R. B., 1955a. The blister beetle genus Linsleya (Coleoptera, Meloidae). American Museum Novitates, 1730: 1-30.

SElander, R. B., 1955b. The proper name for the tribe Calospastini and a correction of generic synonymy (Meloidae). The Coleopterists'Bulletin, 9: 17-19.

SELANDER, R. B., 1956. Geographic variation in the blister beetle Lytta biguttata (Coleoptera, Meloidae). Bulletin of the Brooklyn Entomological Society, 51: 116-127.

Selander, R. B., 1957a. A new Mexican Eupompha (Coleoptera, Meloidae). Entomological News, 68: 123-125.

SELANDER, R. B., 1957b. Descriptions and records of North American Meloidae, I. (Coleoptera). Proceedings of the Entomological Society of Washington, 59(3): 135-141.

SELANDER, R. B., 1957c. A new Nemognatha from southern Mexico (Meloidae). The Coleopterists' Bulletin, 11: 25-28.

Selander, R. B., 1958. Melanism in some Meloidae (Coleoptera) from the Pinacate lava cap in northwestern Sonora, Mexico. Transactions of the Kansas Academy of Sciences, 61: 77-80.

SElANDER, R. B., 1960a. Bionomics, systematics and phylogeny of Lytta, a genus of blister beetles (Coleoptera: Meloidae). Illinois Biological Monographs, 28: i-iv, 1295.

Selander, R. B., 1960b. Restriction of the genus Lytta Fabricius (Meloidae). The Coleopterists'Bulletin, 14: 80-86.

SElander, R. B., 1963. New species and new synonymy in Pyrota Dejean (Coleoptera: Meloidae). The Coleopterists'Bulletin, 17: 33-41. 
SELANDER, R. B., 1964a. Sexual behavior in blister beetles (Coleoptera: Meloidae) I. The genus Pyrota. The Canadian Entomologist, 96: 1037-1081.

SElander, R. B., 1964b. The systematic position of the genus Linsleya (Coleoptera: Meloidae). Proceedings of the Entomological Society of Washington, 66: 216.

SElander, R. B., 1965a. A taxonomic revision of the genus Megetra (Coleoptera: Meloidae) with ecological and behavioral notes. The Canadian Entomologist, 97: 561-580.

SElander, R. B., 1965b. The systematic position of Meloetyphlus, a genus of blind blister beetles (Coleoptera: Meloidae). Journal of the Kansas Entomological Society, 38: 45-55.

SElANDER, R. B., 1981. Evidence for a third larval prey in blister beetles (Coleoptera: Meloidae). Journal of the Kansas Entomological Society, 54: 757-783.

SElANDER, R. B., 1982a. Larval development of blister beetles of the genus Linsleya (Coleoptera: Meloidae). Proceedings of the Entomological Society of Washington, 84: 753-760.

SElander, R. B., 1982b. A revision of the genus Pyrota. I. The Mylabrina group (Coleoptera: Meloidae). Journal of the Kansas Entomological Society, 55: $665-717$.

SELANDER, R. B., 1982c. Sexual behavior, bionomics, and first-instar larvae of the Lauta and Diversicornis groups of Epicauta (Coleoptera: Meloidae). Proceedings of the Entomological Society of Washington, 84: 797-821.

SELANDER, R. B., 1983a. An annotated catalog of blister beetles of the tribe Tetraonycini (Coleoptera, Meloidae). Transactions of the American Entomological Society, 109: 277-293.

SElander, R. B., 1983b. A revision of the genus Pyrota. II. The Postica group (Coleoptera, Meloidae). Proceedings of the Entomological Society of Washington, 85: 69-85.

SElANDER, R. B., 1983c. A revision of the genus Pyrota. III. The pacifica and nobilis groups (Coleoptera, Meloidae). Transactions of the American Entomological Society, 108: 459-478.

SElAnder, R. B., 1983d. A revision of the genus Pyrota. IV. The Tenuicostatis group (Coleoptera, Meloidae). Journal of the Kansas Entomological Society, 56: 1-19.

SElANDER, R. B., 1985a. A new genus of Blister Beetles linking Meloetyphlus, with Tetraonyx (Coleoptera: Meloidae). Journal of the Kansas Entomological Society, 58: 611-619.

SElANDER, R. B., 1985b. Spastomeloe, a new genus of Meloini from Peru (Coleoptera: Meloidae). Journal of the Kansas Entomological Society, 58: 668-685.

SElander, R. B., 1987. The type species of Zonitis Fabricius and the synonymies of $Z$. flava Fabricius and $Z$. ruficollis Frivaldszky (Col., Meloidae). Deutsche Entomologische Zeitschrift (N.F.), 34: 341-350.
SElAnder, R. B., 1991. On the nomenclature and classification of the Meloidae (Coleoptera). Insecta Mundi, 5: 65-94.

Selander, R. B. \& Agafitei, N. J., 1982. First-instar larvae of the Uniforma group of the genus Epicauta (Coleoptera: Meloidae). Proceedings of the Entomological Society of Washington, 84: 138-148.

Selander, R. B. \& Bouseman, J. K., 1960. Meloid beetles of the West Indies. Proceedinsg of the United States National Museum, 111: 197-226.

Selander, R. B. \& Bouseman, J. K., 1962. Supplementary records of meloid beetles (Coleoptera) of the West Indies. Entomological News, 72: 190-191.

Selander, R. B. \& Martínez, A., 1984. A synopsis of the genus Tetraonyx in Argentina (Coleoptera: Meloidae). Proceedings of the Entomological Society of Washington, 86: 913-929.

Selander, R. B. \& Mathieu, J. M., 1969. Ecology, behavior, and adult anatomy of the Albida group of the genus Epicauta (Coleoptera, Meloidae). Illinois Biological Monographs, 41: 1-168.

Selander, R. B. \& Selander, T. C., 1992. A new species and new records of Tetraonyx from Argentina (Coleoptera, Meloidae). Journal of the Kansas Entomological Society, 65(4): 357-368.

Selander, R. B. \& VAurie, P., 1962. A gazetteer to accompany the "Insecta" volumes of the "Biologia Centrali-Americana". American Museum Novitates, 2099: 1-70.

SKINNER, H., 1903. A new variety of Tegrodera (Coleoptera). Entomological News, 14(6): 168.

Skinner, H., 1904. New Meloidae from Arizona. Entomological News, 15: 217.

SkInNER, H., 1906. A new Cantharis. Entomological News, 17: 217.

Spieth, H. T., 1950. The David Rockefeller Mexican Expedition of the American Museum of Natural History. Introductory account. American Museum Novitates, 1454: 1-67.

StaIG, R. A., 1940. The Fabrician types of Insects in the Hunterian Collection at Glasgow University. Coleoptera, part II. Cambridge University Press. 164 págs.

Sturm, J., 1843. Catalog der Kaefer-Sammlung. Kosten des Verfassers, Nürnberg. 386 pp., 6 láms.

Swederus, N. S., 1787. Et nytt genus, och femtio nya species af Insekter beskrifne. Svenska Vetenskaps Akademien Nya Handlingar, 8: 181-201.

Thomson, C. G., 1859. Skandinaviens Coleoptera, Synoptiskt Bearbetade. 1. Conspectus Familiarum et Generum Scandinaviae. Lund. 290 pp.

ThunberG, C. P., 1784. Novae Insectorum species descriptae. Nova Acta Regiae Societatis Scientiarum Upsaliensis, 4: 1-28. 
Thunberg, C. P., 1787. Donationis Thunbergianae 1785 continuatio I. Museum naturalium Academiae Upsaliensis. Parties 1-8, 30-3.

VAn Dyke, E. C., 1928. A reclassification of the genera of North American Meloidae (Coleoptera) and a revision of the genera and species formerly placed in the tribe Meloini, found in America north of Mexico, together with descriptions of new species. University of California Publications in Entomology, 4: 395474, láms. 15-19.

VAN Dyke, E. C., 1929. New species of Meloidae (Coleoptera). Bulletin of the Brooklyn Entomological Society, 24: 127-133.

VAn Dyke, E. C., 1947. New species of Coleoptera from western North America. Pan-Pacific Entomologist, 23: $155-161$.

VAurie, P., 1950. The blister beetles of North Central Mexico (Coleoptera: Meloidae). American Museum Novitates, 1477: 1-68.

VÁzQuez G. L. \& Zaragoza C. S., 1979. Tipos existentes en la colección entomológica del Instituto de Biología, de la Universidad Nacional Autónoma de México. Anales del Instituto de Biología de la Universidad Nacional Autónoma de México, Serie Zoología, 50: 575-632.

VILladA, M., 1901. Catálogo de la colección de coleópteros mexicanos del Museo Nacional, formada y clasificada por el Dr. Eugenio Dugès, Segunda Edición. México. 148 pp.

Villada, M. \& PeÑafiel, A., 1867. Cantharis divirgata (de dos listas). Pág.: 14. En: Herrera, A.; Mendoza, G. 1867. Apuntes para la monografía de los insectos vesicantes indígenas (continúa). Gaceta Médica de México, 3(1): 13-15.

VILLERS, C. DE, 1789. Caroli Linnaei entomologia, faunce Suecica descriptionibus aucta; DD. Scopoli, Geoffroy, De Geer, Fabricii, Schrank \&c. speciebus vel in systemate non enumeratis, vel nuperrime detectis, vel speciebus Gallice Australis locupletata, generum specierumque rariorum iconibus ornata. Tomus primus. Piestre \& Delamollière, Lugduni. $16+765$ pp.

Voet, J. E., 1806. Catalogus Systematicus coleoperorum, vol. 2. Bakhuysen, La Haye. 82 pp.

Walker, F., 1866. Appendix. [List of Coleoptera]. Pp. 309-334 En: Lord, J.K. The naturalist in Vancouver Island and British Columbia. Richard Bentley, London. 375 pp.

Wellman, F. C., 1909. A revision of the genus Calospasta LeConte. Entomological News, 20: 19-25.

Wellman, F. C., 1910a. On the classification of the Lyttidae (Meloidae s. Cantharidae auctt.). Entomological News, 21: 211-222.

Wellman, F. C., 1910b. The generic and subgeneric types of the Lyttidae (Meloidae s. Cantharidae aucit.) (Col.). The Canadian Entomologist, 42: 389-396.
Wellman, F. C., 1910c. On the synonymy of the Meloidae. Deutsche Entomologische Zeitschrift, 1910: 22-26.

Wellman, F. C., 1912. New species of Lyttidae, with notes on described species (Coleoptera). Entomological News, 23: 29-38.

WERnER, F. G., 1943. Revision of the genus Pleuropompha LeConte (Coleop., Meloidae). Psyche, 50: 30-33.

Werner, F. G., 1944. Some new North American species of Epicauta (Coleoptera: Meloidae). Psyche, 50 (1943): 65-73.

Werner, F. G., 1945. A revision of the genus Epicauta in America north of Mexico. Bulletin of the Museum of Comparative Zoology at Harvard College, 95: 421-531.

Werner, F. G., 1949a. Epicauta diversicornis and its allies in the Neotropical Region (Coleop., Meloidae). Psyche, 56(2): 74-80.

Werner, F. G., 1949b. Additions to Epicauta, with new synonymy and a change of names (Coleoptera: Meloidae). Psyche, 56(3): 93-111.

Werner, F. G., 1951. Additions to the nearctic Meloidae (Coleop.). Psyche, 57 (1950): 131-136.

Werner, F. G., 1954a. Pyrota plagiata (Haag) a valid Mexican species (Coleop., Meloidae). Bulletin of the Brooklyn Entomological Society, 49: 102-104.

WerNer, F. G., 1954b. A review of the subgenus Gnathospasta of the genus Epicauta (Meloidae). The Coleopterists'Bulletin, 8: 25-27.

Werner, F. G., 1955. Studies in the genus Epicauta of the North American continent (Coleop., Meloidae) I - The Caviceps-group. Bulletin of the Brooklyn Entomological Society, 50: 1-12.

Werner, F. G., 1957b. Epicauta dugesi a valid species (Coleoptera: Meloidae). Psyche, 64: 107-108.

Werner, F. G., 1958. Studies in the genus Epicauta of the North American continent (Meloidae) II. The Uniforma-group. The Coleopterists'Bulletin, 12: 1-19.

Werner, F. G., 1973. Three new Epicauta from Mexico (Coleoptera: Meloidae). Proceedings of the Entomological Society of Washington, 75: 458-463.

Werner, F. G., 1974. A new genus of primitive Meloidae from West Texas (Coleoptera). Psyche, 81(1): 147-154.

Werner, F. G., Enns, W. R. \& PArker, F. H., 1966. The Meloidae of Arizona. The University of Arizona Agricultural Experiment Station Technical Bulletin, 175: 1-96.

WicKHAM, H. F., 1905. New species of Coleoptera from the western United States. Second paper. The Canadian Entomologist, 37: 165-171.

Woodhouse, J., 1800. Of American blistering flies. Medical Repository, 3(2): 213-214. 
YANEGA, D. \& LeSCHEN, R. A. B., 1994. Beetles associated with bee nests (Hymenoptera: Apidae) in Chiapas. México, with descriptions of the immature stages of Vanonus balteatus Werner (Coleoptera: Aderidae, Endomychidae, Meloidae). The Coleopterists'Bulletin, 48(4): 355-360.

Zaragoza-Caballero, S., 1999. Eugenio Dugès: un precursor de la Entomología en México. Dugesiana, 6: 1-26.

Zaragoza-Caballero, S. \& Velasco-de León, M. P., 2001. First record of Epicauta (Coleoptera, Meloidae) of the Atotonilco El Grande Formation (resumen). Pág. 137. En: Memories of North American Paleontological Convention Berkeley, California: Paleobios, 21, supplement 2.
Zaragoza-Caballero, S. \& Velasco-de León, P., 2003. Una especie nueva de Epicauta (Coleoptera: Meloidae) del Plioceno del Estado de Hidalgo, México. Revista Mexicana de Ciencias Geológicas, 20: 154-159.

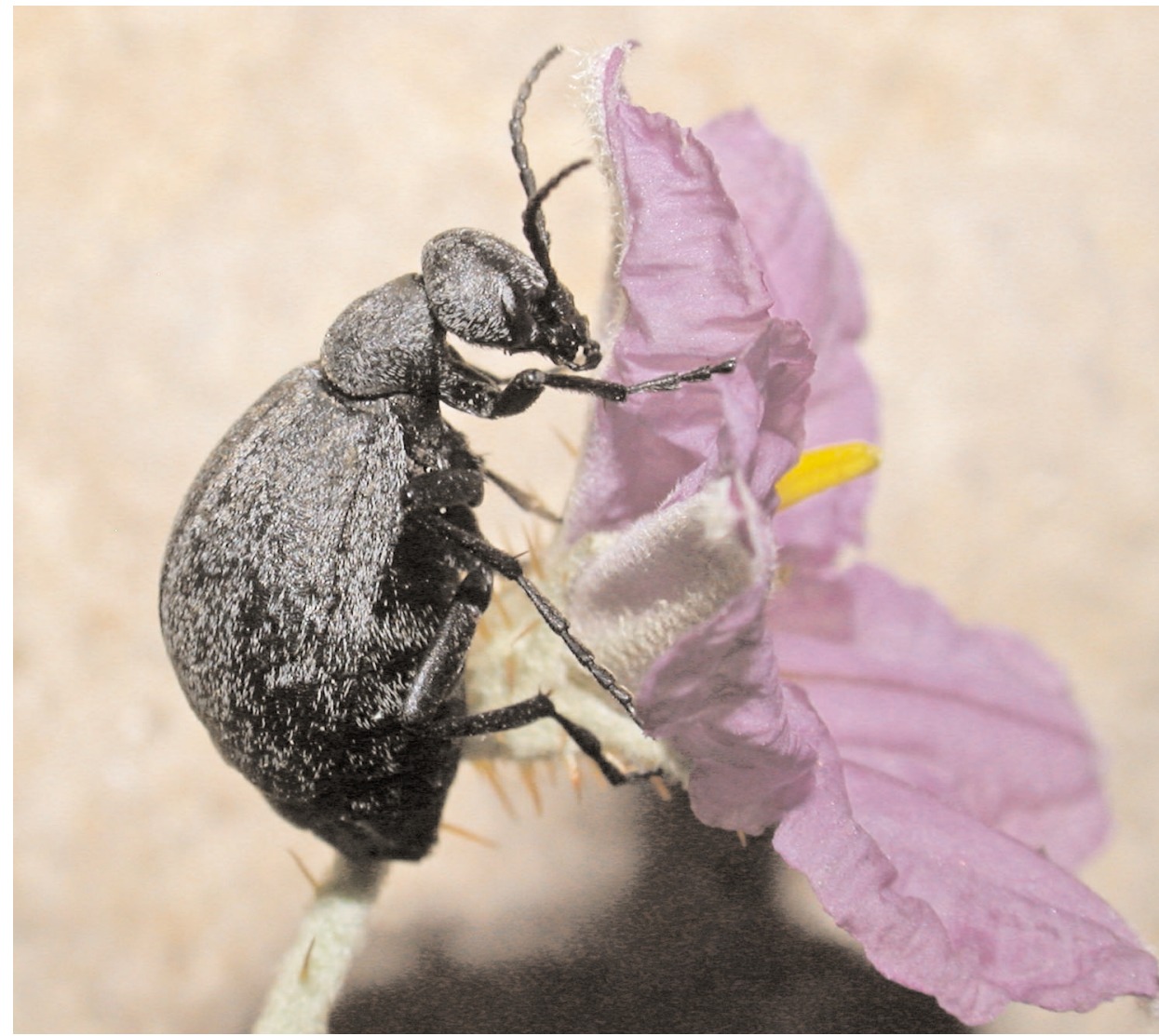

Epicauta cardui. México: Jalisco: 8 km al SE de Ojuelos de Jalisco.

Recibido, 2-II-2007

Aceptado, 21-XI-2007

Publicado, 27-XII-2007 
Índice de nombres de especies, géneros y subgéneros en uso.

\begin{tabular}{|c|c|c|}
\hline adona (Epicauta) & (LPtcuata) & - \\
\hline eona (Epicauta) & assitarsis (Epicauta) & sueta (Epicauta) \\
\hline tta . & ibrata (Lytta) & (Nemognatha) \\
\hline$a$ (Epicauta) & oceicincta (Epicauta) & (Pyrota) \\
\hline ana (Pyrota) & ipraeola (Epicauta). & thmica (Epicauta) \\
\hline icauta) ..... & irvicornis (Epi & jimenezi (Epicauta) \\
\hline (En & > & koltzei (Lytta) ............. \\
\hline olineata (Epicauta) …………...... & eceptiva (Pyrota) & labialis (Epicauta) \\
\hline & ens (Tetraonyx) & evicornis (Epicauta) \\
\hline & 07 & \\
\hline & & (Epicauta) \\
\hline & & $t a t$ \\
\hline & & ytta) \\
\hline & & uta). \\
\hline & & (Epicauta) \\
\hline & auta) & uta) \\
\hline & & picauta) \\
\hline 4 & icauta) & \\
\hline (Zonitic) & & \\
\hline & & the \\
\hline a) & & ognatha) \\
\hline & & …………...... \\
\hline es) & s) $\ldots . .$. & \\
\hline & & (Epicauta) \\
\hline & & \\
\hline & & \\
\hline & & \\
\hline & & a) \\
\hline & & 1) \\
\hline$n y x)$ & & ta) \\
\hline & & \\
\hline ta) & & \\
\hline & & \\
\hline & & ognatha \\
\hline & & \\
\hline gnatha) & gnatha) & \\
\hline zatha) & & oa (Epicauta) \\
\hline ta) & $n y x)$ & ………... \\
\hline & auta). & (Epicauta) \\
\hline ra) & ıpha) & \\
\hline & & $y t t a)$ \\
\hline & & ium) \\
\hline & & \\
\hline zatha) & & ta) \\
\hline & & \\
\hline & itha) & a) \\
\hline & & \\
\hline & $y x)$ & a) \\
\hline uta) & 3 & Meloe) \\
\hline & $f u$ & tha \\
\hline mognatha) & & \\
\hline uta) & 2 & a) \\
\hline icauta) & Epicauta) & is (Nemognatha) ... \\
\hline 1 & ……….... & (Epicauta) .. \\
\hline$D_{1}$ & Epicauta) & nigritibialis (Epicauta) \\
\hline & & nigromaculata (Zonitis) \\
\hline & & tata (Pyrota) \\
\hline ra (Lytta) & & $y t t a)$ \\
\hline & & \\
\hline
\end{tabular}




\begin{tabular}{|c|c|c|}
\hline iveolineata (Epicauta) & yrota & tarasca (Zonitis) \\
\hline obilis (Pyrota) & quadrimaculata (Lytta) ................... 170, 218 & Tegrodera ............... \\
\hline besa (Epicauta) & quadrinervata (Pyrota) & temexa (Epicauta) \\
\hline ccipitalis (Epicauta) & reticulata (Pleuropasta) ... & tenebrosa (Epicauta) \\
\hline cellata (Epicauta) & Rhyphonemognatha . & tenebrosa (Rhyphonemognata) \\
\hline chrea (Epicauta) & rileyi (Epicauta) & tenella (Epicauta) \\
\hline tomaculatus (Tetraonyx) & rufa (Rhyphonemognata) ..... & tenuemarginata (Ep \\
\hline acifica (Epicauta) & rufipennis (Epicauta). & tenuicornis (Epicauta) \\
\hline acifica (Pyrota) & rugulipennis (Pyrota) ....... & tenuicostatis (Pyrota) \\
\hline allens (Nemognatha) & sallei (Tetraonyx) & tenuilineata \\
\hline oalpalis (Pyrota) & sanctoruensis (Epicauta). & teresa (Epicauta) \\
\hline aralytta $\ldots \ldots . . .$. & sanguinea (Lytta) ............... & terminalis (Eupompha) \\
\hline arazonitis ...................... & lis (Rhyphonemognata) .... 1' & terminata (Epicauta) \\
\hline ardalis (Epicauta) & ayi (Zonitis) & is (Pyrota) \\
\hline Pauronemognatha & scitula (Lytta) & Tetraonyx \\
\hline edalis (Epicauta) & scituloid & texana (Epicauta) \\
\hline oeninsularis (Lytta) & scutellaris (Nemognatha) ... & Treiodous ................. \\
\hline oensylvanica (Epicauta) & scutellaroides (Nemognatha) & tricostata (Epicauta) \\
\hline 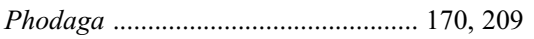 & segmenta (Epicauta) ............ & tripartita (Epicauta) \\
\hline phoenix (Epicauta) .... & selanderi (Nemognatha) & triquetra (Epi \\
\hline diazata (Nemognatha) & selanderorum (Epicauta) & terica (Pyrota) \\
\hline plagiata (Pyrota) & selloa $(N$ & unicalcarata \\
\hline Pleuropasta & senilis (Epicauta) ............ & uniforma (Epicauta) \\
\hline ploribunda (Zonitis) & sericans (Epicauta) & unilineata (Epicauta) \\
\hline plumbea (Lytta) ....... & sexguttatus (Tetraonyx) & variabilis (Lytta) \\
\hline polingi (Epicauta) & singularis (Epicauta) ........... & vaurieae (Pseudozonitis) \\
\hline Poreospasta .................. & sonorae (Lytta) & virgulata (Epicauta) \\
\hline postica (Pyrota). & Spastonyx. & viridis (Eupompha) \\
\hline Pronemognatha & stigmata (Epicauta) & vitticollis (Epicauta) \\
\hline prosopidis (Epicauta) & straba (Epic & vizcaina (Eupompha) . \\
\hline proteus (Lytta) & strigulosus (Meloe) & vulnerata (Lytta) \\
\hline Pseudozonitis . & suavissima (Linsleya) & wenzeli (Eup \\
\hline punctata (Megetra) & subatra (Epicauta) ............... & wislizeni (Cysteodemus) \\
\hline punctata (Pyrota) & sublineata (Epicauta) & xanthochroa (Zonitis) \\
\hline puncticollis (Epicauta) & subrubra (Epicauta) & Zonitis \\
\hline punctum (Epicauta) & sulcifrons (Eupompha) & zonitoides (Nemognatha) \\
\hline urpurea (Epicauta) & tarasca (Epicauta) & \\
\hline
\end{tabular}

